\title{
Atlantic Ocean Circulation at the Last Glacial Maximum: Inferences from Data and Models
}

\author{
by \\ Holly Janine Dail \\ Submitted in partial fulfillment of the requirements for the degree of \\ Doctor of Philosophy \\ at the \\ MASSACHUSETTS INSTITUTE OF TECHNOLOGY \\ and the \\ WOODS HOLE OCEANOGRAPHIC INSTITUTION \\ September 2012 \\ (c) Holly Janine Dail, MMXII. All rights reserved. \\ The author hereby grants to MIT and WHOI permission to reproduce and \\ to distribute publicly paper and electronic copies of this thesis document in \\ whole or in part in any medium now known or hereafter created.
}

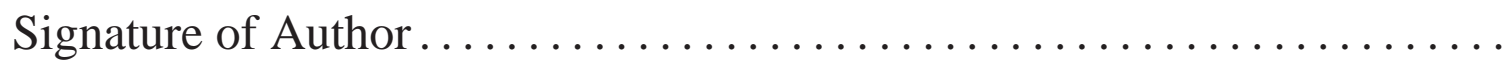

Joint Program in Oceanography - Massachusetts Institute of Technology / Woods Hole Oceanographic Institution

August 14, 2012

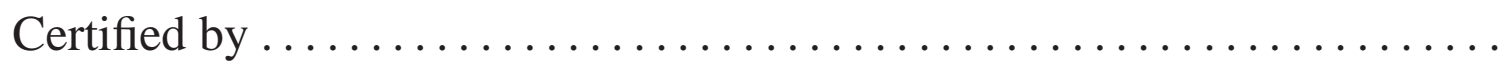

Carl I. Wunsch

Cecil and Ida Green Professor of Physical Oceanography Massachusetts Institute of Technology

Thesis Supervisor

Accepted by

Karl R. Helfrich

Senior Scientist, Woods Hole Oceanographic Institution Chair, Joint Committee for Physical Oceanography 


\title{
Atlantic Ocean Circulation at the Last Glacial Maximum: Inferences from Data and Models
}

\author{
by \\ Holly Janine Dail
}

\begin{abstract}
Submitted to the Joint Program in Oceanography,
Massachusetts Institute of Technology and the Woods Hole Oceanographic Institution on August 14, 2012, in partial fulfillment of the requirements for the degree of

Doctor of Philosophy
\end{abstract}

\begin{abstract}
This thesis focuses on ocean circulation and atmospheric forcing in the Atlantic Ocean at the Last Glacial Maximum (LGM, 18-21 thousand years before present). Relative to the pre-industrial climate, LGM atmospheric $\mathrm{CO}_{2}$ concentrations were about $90 \mathrm{ppm}$ lower, ice sheets were much more extensive, and many regions experienced significantly colder temperatures. In this thesis a novel approach to dynamical reconstruction is applied to make estimates of LGM Atlantic Ocean state that are consistent with these proxy records and with known ocean dynamics.

Ocean dynamics are described with the MIT General Circulation Model in an Atlantic configuration extending from $35^{\circ} \mathrm{S}$ to $75^{\circ} \mathrm{N}$ at $1^{\circ}$ resolution. Six LGM proxy types are used to constrain the model: four compilations of near sea surface temperatures from the MARGO project, as well as benthic isotope records of $\delta^{18} \mathrm{O}$ and $\delta^{13} \mathrm{C}$ compiled by Marchal and Curry; 629 individual proxy records are used. To improve the fit of the model to the data, a least-squares fit is computed using an algorithm based on the model adjoint (the Lagrange multiplier methodology). The adjoint is used to compute improvements to uncertain initial and boundary conditions (the control variables). As compared to previous model-data syntheses of LGM ocean state, this thesis uses a significantly more realistic model of oceanic physics, and is the first to incorporate such a large number and diversity of proxy records.

A major finding is that it is possible to find an ocean state that is consistent with all six LGM proxy compilations and with known ocean dynamics, given reasonable uncertainty estimates. Only relatively modest shifts from modern atmospheric forcing are required to fit the LGM data. The estimates presented herein succesfully reproduce regional shifts in conditions at the LGM that have been inferred from proxy records, but which have not been captured in the best available LGM coupled model simulations. In addition, LGM benthic $\delta^{18} \mathrm{O}$ and $\delta^{13} \mathrm{C}$ records are shown to be consistent with a shallow but robust Atlantic meridional overturning cell, although other circulations cannot be excluded.
\end{abstract}

Thesis Supervisor: Carl I. Wunsch

Title: Cecil and Ida Green Professor of Physical Oceanography 


\section{Acknowledgments}

First, I thank Carl Wunsch, my advisor. Carl has impeccable taste in choosing scientific problems, and the wisdom, perspective, and fortitude to make meaningful contributions to even the most difficult. I have benefited greatly from his patient guidance and clear vision.

My thesis committee has been invaluable. Patrick Heimbach was incredibly helpful throughout with both adjoint modeling and with modern oceanography; I owe him much. Olivier Marchal graciously shared his extensive knowledge about proxy records and helped define appropriate assumptions for modeling isotopes. From Peter Huybers I have learned much about how to frame a problem effectively, and how to reduce it to its simplest form. Finally, Jake Gebbie has generously shared his wisdom from extensive experience with nearly all the subjects addressed in the thesis, and provided much needed advice at critical stages.

Many useful contributions have been made to this work from other collaborators. In the ECCO group, Gaël Forget's state estimation expertise has been of great value, and early help from Constantinos Evangelinos is appreciated. Jean-Michel Campin has been patient, gracious with his time, and wonderfully helpful. Dan Amrhein generously read an earlier version of the thesis and provided many helpful comments. I also thank Delia Oppo, Bill Curry, André Paul, Martin Losch, Bette Otto-Bliesner, Nan Rosenbloom, and Esther Brady.

The Academic Programs Office at WHOI and the administrative staff at MIT have been a great resource and have provided a human face to the program. I especially thank Marsha Armando, Julia Westwater, Annie Doucette, Beth MacEachran, and Mary Elliff.

Classes and generals would have been much less fun and a lot more challenging without Pat, Rachel, Rebecca, Cim, Laura, and Ryan. Early office mates Stephanie, Yohai, and Matt were, and continue to be, great mentors. Brian, Jinbo, Martha, and Sophie C. have been great colleagues too.

Finishing this thesis involved some dark days and I am grateful to my friends and family who supported me through it all. I especially thank my parents and my sister who supported me, as always, through thick and thin. I also owe thanks to friends Kate, Leeann, Kathy, Margaret, Shaun, Debbie, and Kurt.

I dedicate this thesis to my family - to my husband Alan, who is an incredible partner, and to Norah, who is very patiently teaching us what is truly important.

Financial support. I gratefully acknowledge the following financial support. Primary support was provided by a National Defense Science and Engineering Graduate Fellowship and two National Science Foundation awards: Award \#OCE-0645936: "Beyond the Instrumental Record: the Case of Circulation at the Last Glacial Maximum" and Award \#OCE-1060735: "Collaborative Research: Beyond the Instrumental Record - the Ocean Circulation at the Last Glacial Maximum and the de-Glacial Sequence". Important secondary support came from the National Ocean Partnership Program and the National Aeronautics and Space Administration via the ECCO effort at MIT. 
Computational resources. This work required significant computer time. Preliminary work relied on resources at NCAR, on local resources in the ECCO group at MIT, and on the "Swell" departmental cluster at Harvard. The final results were all computed on the National Aeronautics and Space Administration Pleiades machine under the ECCO2 project allocation. This is the most professionally run compute resource I have used in 15 years of parallel computing.

Providers of data and model output. I acknowledge the members of the MARGO project for providing reconstructed LGM SST and sea ice estimates; O. Marchal and W. Curry for providing LGM and Holocene benthic isotope data; the PMIP2 international modeling groups for providing their model output for analysis; the Laboratoire des Sciences du Climat et de l'Environnement for collecting and archiving the PMIP2 output; W.R. Peltier for providing ICE-5G bathymetry and coastline estimates; A. LeGrande and G. Schmidt for providing a modern database of $\delta^{18} \mathrm{O}_{\text {water }}$ measurements; and the GEOSECS project for making available modern water column $\delta^{13} \mathrm{C}_{\mathrm{DIC}}$ measurements. 


\section{Abbreviated Contents}

$\begin{array}{ll}\text { List of Figures } & 13\end{array}$

$\begin{array}{ll}\text { List of Tables } & 17\end{array}$

$\begin{array}{ll}\text { Acronyms used in the thesis } & 19\end{array}$

1 Introduction $\quad 21$

2 Last Glacial Maximum Atlantic $\quad 31$

3 State estimation methodology with application to modern circulation $\quad 85$

4 Upper ocean conditions at the Last Glacial Maximum 145

5 Deep ocean conditions at the Last Glacial Maximum 191

6 Discussion 209

$\begin{array}{ll}\text { A MARGO uncertainty assignments } & 217\end{array}$

$\begin{array}{ll}\text { Bibliography } & 221\end{array}$ 


\section{Contents}

$\begin{array}{ll}\text { List of Figures } & 13\end{array}$

$\begin{array}{ll}\text { List of Tables } & 17\end{array}$

$\begin{array}{ll}\text { Acronyms used in the thesis } & 19\end{array}$

1 Introduction $\quad 21$

1.1 The Last Glacial Maximum . . . . . . . . . . . . . . . . . . . 22

1.2 Dynamical reconstruction . . . . . . . . . . . . . . . 24

1.3 Terminology ........................... 27

1.4 Structure of the thesis f . . . . . . . . . . . . 28

2 Last Glacial Maximum Atlantic $\quad 31$

2.1 Geography and atmospheric State . . . . . . . . . . . . . 32

2.2 Proxies of LGM ocean state . . . . . . . . . . . . . . 38

2.2.1 Introduction to potential uncertainties and biases . . . . . . . . 39

2.2.2 Upper ocean state . . . . . . . . . . . . . . . . . 42

2.2.3 Deep ocean state ..................... 54

2.3 Forward models of ocean state . . . . . . . . . . . . . . 70

2.3.1 The Paleoclimate Modeling Intercomparison Project . . . . . . . 71

2.3.2 Isotope models . . . . . . . . . . . . . . . . . 74

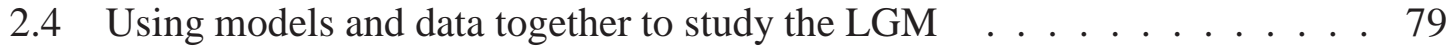


2.4.1 Quantitative model-data comparison . . . . . . . . . . 80

2.4.2 Synthesizing models and data ............. 81

2.5 Selection of data constraints for the thesis . . . . . . . . . . . . 84

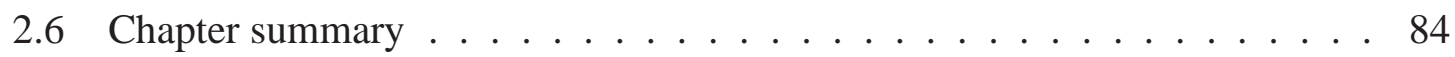

3 State estimation methodology with application to modern circulation 85

3.1 Forward model configuration . . . . . . . . . . . . . . 86

3.1.1 Numerics and domain . . . . . . . . . . . . 86

3.1.2 Initial and boundary conditions . . . . . . . . . . . . 89

3.2 Ocean state estimation $\ldots \ldots \ldots \ldots$. . . . . . . . . . . . 90

3.2.1 A least-squares problem and the method of Lagrange multipliers . . 91

3.2.2 Data constraints and uncertainties ............. 96

3.2.3 Control variables and uncertainties . . . . . . . . . . . . . 98

3.2.4 A focus on equilibrium ocean estimation . . . . . . . . . . 101

3.3 State estimation with modern T/S information: the Modern_TS estimate . . 102

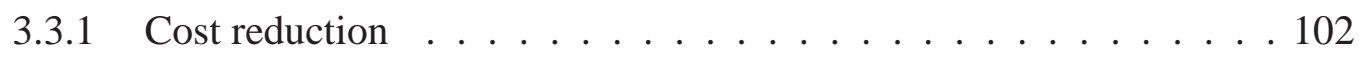

3.3.2 Consistency of the state estimate . . . . . . . . . . . . . 104

3.3.3 Inferred properties . . . . . . . . . . . . . 112

3.4 Modern state estimation with $\delta^{13} \mathrm{C}_{\text {DIC }}$ and $\delta^{18} \mathrm{O}_{\text {water }}$ observations $\ldots \ldots 117$

3.4.1 $\delta^{18} \mathrm{O}_{\text {water }}$ : forward modeling and state estimation . . . . . . . . 119

3.4.2 $\delta^{13} \mathrm{C}_{\mathrm{DIC}}$ : forward modeling and state estimation $\ldots . . \ldots 122$

3.4.3 The Modern_Iso estimate . . . . . . . . . . . . . . . . . . 126

3.5 Extending state estimate length: the search for a seasonal steady state . . 129

3.5.1 Asynchronous timestepping . . . . . . . . . . . . 129

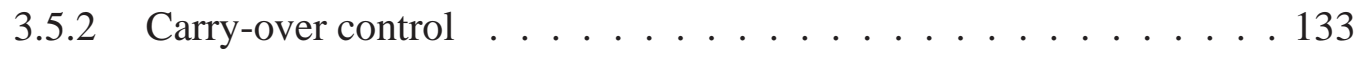

3.5.3 Analysis of a longer state estimate: Modern_80yrs . . . . . . . . 135

3.5.4 Current limitations in equilibrium ocean estimation . . . . . . . . 142

3.6 Chapter summary . . . . . . . . . . . . . . . . . . 144 
4 Upper ocean conditions at the Last Glacial Maximum

4.1 Forward model . . . . . . . . . . . . . . . . . . . . . . . . . . . . 147

4.1 .1 First guess initial conditions _ . . . . . . . . . . . 148

4.1.2 First guess atmospheric forcing . . . . . . . . . . . . 149

4.2 Adjoint model . . . . . . . . . . . . . . . . . . 150

4.3 Exploration of assumptions . . . . . . . . . . . . . . . 152

4.3.1 Using all MARGO data as published: LGM_AllSSTs . . . . . . . 153

4.3.2 Using subsets of data: LGM_NoForams and LGM_Forams . . . . 156

4.3.3 Using larger atmospheric uncertainties: LGM_ForamsX2 . . . . 158

4.4 A best estimate: LGM_Upper . . . . . . . . . . . . . . . . . . . . 158

4.4 .1 Assumptions . . . . . . . . . . . . . . . . . . 159

4.4.2 Consistency of the state estimate . . . . . . . . . . . 161

4.4 .3 Analysis of solution . . . . . . . . . . . . 167

4.5 Comparison to PMIP2 models . . . . . . . . . . . . . . . 176

4.5.1 Near sea surface temperatures $\ldots \ldots \ldots . \ldots 176$

$4.5 .2 \quad$ Seasonality . . . . . . . . . . . . . . . 181

4.5 .3 Wind field . . . . . . . . . . . . . . . . . . 181

4.5.4 Sea ice distributions . . . . . . . . . . . . . . . 185

4.6 Chapter summary . . . . . . . . . . . . . . . . . . 186

5 Deep ocean conditions at the Last Glacial Maximum 191

$5.1 \delta^{18} \mathrm{O}$ and $\delta^{13} \mathrm{C}$ data from benthic foraminifera . . . . . . . . . . 192

5.2 Forward model and state estimation configuration . . . . . . . . . 193

5.3 The search for an acceptable solution . . . . . . . . . . . . . 195

5.4 Analysis of LGM_Deep10yr and LGM_Deep . . . . . . . . . . . . 196

5.4.1 NSSTs and the MOC . . . . . . . . . . . . . . . 196

5.4.2 Modeled versus observed $\delta^{13} \mathrm{C} \ldots \ldots \ldots \ldots$. . . . . 200

5.4.3 Modeled versus observed $\delta^{18} \mathrm{O} \ldots \ldots \ldots$. . . . . . . 203

5.5 Comparison to previous studies . . . . . . . . . . . . . 206 


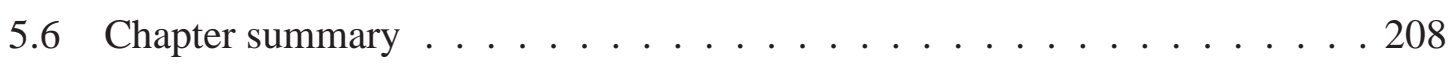

6 Discussion $\quad 209$

6.1 A vision for ocean state estimation and equilibrium ocean estimation . . . . 209

6.2 Novel contributions of the thesis . . . . . . . . . . . . . . 211

6.3 Limitations and opportunities for future progress . . . . . . . . . . . 212

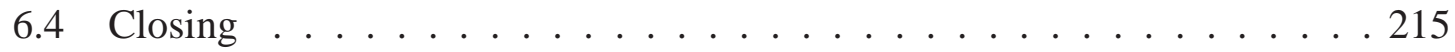

$\begin{array}{lll}\text { A MARGO uncertainty assignments } & 217\end{array}$

$\begin{array}{ll}\text { Bibliography } & 221\end{array}$ 


\section{List of Figures}

2-1 LGM coastlines, ice sheets, and temperatures (from Braconnot et al. 2012) . 33

2-2 ICE-4G and ICE-5G reconstructions of Laurentide ice sheet (from Li and Battisti 2008) . . . . . . . . . . . . . . . . 33

2-3 Maps of modern-LGM NSST differences (WOA'09 - MARGO) . . . . . . 46

2-4 NSST differences (WOA'09 - MARGO) plotted against latitude . . . . . . 47

2-5 LGM sea ice distributions (after De Vernal et al. 2006) . . . . . . . . . . 52

2-6 LGM benthic $\delta^{18} \mathrm{O}_{\text {calcite }}$ and $\delta^{13} \mathrm{C}_{\text {calcite }}$ proxy records with core depths . . . . 57

2-7 LGM pore-fluid measurements . . . . . . . . . . . . 63

2-8 Modern $\epsilon_{\mathrm{Nd}}$ and neodymium concentration (after Lacan et al. 2012) . . . 64

2-9 Atlantic distributions of inferred Holocene and LGM seawater cadmium (from Marchitto and Broecker 2006) . . . . . . . . . . . . . . . . . 67

2-10 Scatter plots of modeled vs. observed LGM $\delta^{13} \mathrm{C}$ (after Hesse et al. 2011) . 78

3-1 Evolution of data and control costs over 26 iterations . . . . . . . . 103

3-2 CDFs of normalized T/S misfits for Modern_TS and OCCA . . . . . . . . 105

3-3 CDFs of normalized atmospheric control variables for Modern_TS . . . . . 107

3-4 CDFs of other normalized control variables for Modern_TS . . . . . . . . . 107

3-5 Maps of Modern_TS NSST and NSSS and misfits to OCCA . . . . . . . . 108

3-6 Maps of Modern_TS $300 \mathrm{~m} \mathrm{T/S}$ and misfits to OCCA . . . . . . . . . 110

3-7 Maps of Modern_TS $4000 \mathrm{~m}$ T/S and misfits to OCCA . . . . . . . . . 111

3-8 Velocity maps at 55 and $1160 \mathrm{~m}$ for Modern_TS with misfits to OCCA . . . 113 
3-9 Mean dynamic topography in Modern_TS and the EGM estimate . . . . . 115

3-10 MOC and $29^{\circ} \mathrm{N}$ transport decomposition in Modern_TS and OCCA . . . . 115

3-11 Meridional heat and freshwater transport in Modern_TS . . . . . . . . . 116

3-12 Sea ice fraction maps in February and August in Modern_TS . . . . . . . 118

3-13 Modern sea ice climatology (from Maurer 2007) . . . . . . . . . . . 118

3-14 Distribution of Schmidt et al. [1999] $\delta^{18} \mathrm{O}_{\text {water }}$ observations . . . . . . . . 121

3-15 Distribution of GEOSECS $\delta^{13} \mathrm{C}_{\text {DIC }}$ observations . . . . . . . . . . . 125

3-16 CDFs of normalized $\delta^{18} \mathrm{O}_{\text {water }}$ and $\delta^{13} \mathrm{C}_{\text {DIC }}$ misfits for Modern_Iso . . . . 127

3-17 Modern_Iso $\delta^{13} \mathrm{C}_{\text {DIC }}$ map at $2730 \mathrm{~m}$ with GEOSECS data overlaid . . . . . 128

3-18 Evaluation of asynchronous timestepping to accelerate optimization . . . 132

3-19 Carry over controls as an effective means to extend state estimate length . . 134

3-20 Modern_80yrs $\delta^{13} \mathrm{C}_{\text {DIC }}$ map at $2730 \mathrm{~m}$ with GEOSECS data overlaid . . . . 136

3-21 CDFs of normalized $\delta^{18} \mathrm{O}_{\text {water }}$ and $\delta^{13} \mathrm{C}_{\text {DIC }}$ for Modern_80yrs . . . . . . 137

3-22 MOC and $29^{\circ} \mathrm{N}$ transport decomposition in short and long state estimates . 139

3-23 Improvement in normalized NSST/NSSS misfits with optimization . . . . . 140

3-24 Improvement in normalized T/S misfits at $1100 \mathrm{~m}$ with optimization . . . . 141

4-1 Modern and ICE-5G LGM bathymetries . . . . . . . . . . . . . . . 147

4-2 CDF of the normalized misfit of NSSTs in LGM_AllSSTs to MARGO. . . 154

4-3 Fit of first guess and LGM_AllSSTs estimates to MARGO with explanation of wheel diagrams . . . . . . . . . . . . . . . . . . . . 154

4-4 Fit of LGM_NoForams and LGM_AllSSTs to MARGO . . . . . . . . . . 157

4-5 Fit of LGM_Forams and LGM_AllSSTs to MARGO . . . . . . . . . . . . 157

4-6 Fit of LGM_ForamsX2 and LGM_Forams to MARGO . . . . . . . . . . 159

4-7 Fit of LGM_Upper and LGM_AllSSTs to MARGO . . . . . . . . . . . . 162

4-8 Misfit versus latitude for LGM_Upper and WOA'09 to MARGO . . . . . 163

4-9 Maps of normalized misfits of LGM_Upper to MARGO . . . . . . . . . . . 164

4-10 CDFs of normalized atmospheric control variables for LGM_Upper . . . 166

4-11 CDFs of other normalized control variables for LGM_Upper . . . . . . . 166 
4-12 LGM_Upper NSST maps and difference with WOA'09 . . . . . . . 168

4-13 Sea ice fraction in LGM_Upper and difference from modern . . . . . . 170

4-14 Comparison of LGM_Upper sea ice presence to sea ice proxies . . . . . . 171

4-15 Mean dynamic topography in LGM_Upper and Modern_TS . . . . . . . . 172

4-16 AMOC and 29 $\mathrm{N}$ transports in OCCA and LGM_Upper . . . . . . . . 174

4-17 Atmospheric adjustments in LGM_Upper and comparison with modern . . 175

4-18 NSSTs in PMIP2 and LGM_Upper LGM simulations . . . . . . . . 177

4-19 Maps of misfit of PMIP2 NSSTs to MARGO foram assemblages . . . . 178

4-20 Bar graphs of mean cost for LGM_Upper and PMIP2 models . . . . . . . 180

4-21 Difference between Aug. and Feb. NSST in PMIP2 and LGM_Upper . . . . 182

4-22 Maps of PMIP2 and LGM_Upper winds . . . . . . . . . . . . 183

4-23 Maps of LGM-modern differences for PMIP2 and LGM_Upper winds . . 184

4-24 Winter sea ice in PMIP2, LGM_Upper, and proxy records . . . . . . . . 187

4-25 Summer sea ice in PMIP2, LGM_Upper, and proxy records . . . . . . . 188

5-1 Fit of LGM_Upper and LGM_Deep10yr against all proxies _ . . . . . . 197

5-2 Fit of LGM_Deep10yr and LGM_Deep against all proxies . . . . . . . . . 197

5-3 Maps of NSSTs and anomalies in LGM_Deep reveal model drifts . . . . . 198

5-4 AMOC and $29^{\circ} \mathrm{N}$ transports in OCCA, LGM_Deep10yr, and LGM_Deep . . 201

5-5 Scatter plots of $\delta^{13} \mathrm{C}_{\text {calcite }}$ model-data fit . . . . . . . . . . 202

5-6 Maps of LGM_Deep $\delta^{13} \mathrm{C}_{\text {calcite }}$ with MC08 records at 12 levels _ . . . . . 204

5-7 Scatter plots of $\delta^{18} \mathrm{O}_{\text {calcite }}$ model-data fit . . . . . . . . . . . 205

5-8 Maps of LGM_Deep $\delta^{18} \mathrm{O}_{\text {calcite }}$ with MC08 records at 12 levels $\ldots . . .207$

A-1 Histograms of MARGO uncertainties $\ldots \ldots \ldots \ldots \ldots \ldots$ 


\section{List of Tables}

2.1 Proxy records included in LGM state estimates . . . . . . . . . . . . 39

2.2 PMIP2 model configurations. . . . . . . . . . . . . . . . . 72

3.1 Model parameters used in all state estimates. . . . . . . . . . . . . . 87

3.2 Modern atmospheric control uncertainties. . . . . . . . . . . . . 100

3.3 Full list of control variables used in the thesis. . . . . . . . . . . . . 122

3.4 AMOC transports in long-running state estimates, modern configuration. . . 137

4.1 LGM atmospheric control uncertainties. . . . . . . . . . . . . . . 152 


\title{
Acronyms used in the thesis
}

With references where appropriate

\author{
AABW AntArctic Bottom Water \\ AMOC Atlantic Meridional Overturning Circulation \\ CCSM Community Climate System Model [Otto-Bliesner et al., 2006] \\ CDF Cumulative Distribution Function \\ CLIMAP Climate: Long range Investigation, Mapping, and Prediction [CLIMAP \\ Project Members, 1976] \\ DIC Dissolved Inorganic Carbon \\ DWBC Deep Western Boundary Current \\ ECCO Estimating the Circulation and Climate of the Ocean [Wunsch and Heim- \\ bach, 2007] \\ EOE Equilibrium Ocean Estimate \\ GEOSECS Geochemical Ocean Sections Study \\ GNAIW Glacial North Atlantic Intermediate Wwater \\ H1/H2 Heinrich event 1/2 \\ LGM Last Glacial Maximum \\ MARGO Multi-proxy Approach for the Reconstruction of the Glacial Ocean surface \\ [Waelbroeck et al., 2009] \\ MIT GCM Massachusetts Institute of Technology General Circulation Model [Adcroft \\ et al., 2004] \\ NADW North Atlantic Deep Wwater \\ OCCA OCean Comprehensible Atlas [Forget, 2010]
}




$\begin{array}{ll}\text { PI } & \text { Pre Industrial } \\ \text { PMIP } & \text { Paleoclimate Modelling Intercomparison Project [Braconnot et al., 2007a] } \\ \text { NCEP/NCAR } & \text { National Centers for Environmental Prediction / National Center for } \\ & \text { Atmospheric Research Reanalysis [Kalnay and coauthors, 1996] } \\ \text { NSST } & \text { Near Sea Surface Temperature } \\ \text { NSSS } & \text { Near Sea Surface Salinity } \\ \text { SSS } & \text { Sea Surface Salinity (directly at the surface) } \\ \text { SST } & \text { Sea Surface Temperature (directly at the surface - the "skin" temperature) } \\ \text { T/S } & \text { Temperature and Salinity } \\ \text { WOA } & \text { World Ocean Atlas }\end{array}$




\section{Chapter 1}

\section{Introduction}

The LGM is the period of maximum extent of terrestrial ice sheets during the most recent major glaciation in Earth's history. Ice sheet extent appears to have been relatively stable over the period of 26.5 to 19 or 20 thousand years before present (26.5-19 or 20 kyr bp) [Clark et al., 2009], and sea level during this period was relatively stable at about $125 \mathrm{~m}$ below modern levels [Clark et al., 2009]. Atmospheric $\mathrm{CO}_{2}$ concentrations were also relatively stable at about 190 parts per million by volume (ppmv) during the LGM, as compared to about 280 ppmv during pre-industrial times [Schmitt et al., 2012].

Although some aspects of LGM climate are relatively well understood, the atmosphere and ocean circulation at the LGM remain particularly uncertain (see Chapter 2 for a thorough review). As there are relatively large numbers of proxy records available for the LGM Atlantic Ocean, there is a particularly good opportunity to better constrain conditions in this region. In this thesis, the state of the Last Glacial Maximum (LGM) Atlantic Ocean is reconstructed through a combination of proxy evidence and a dynamical understanding of ocean circulation. This work is guided by the following three lines of scientific inquiry.

1. Are there one or more Atlantic Ocean states that are consistent with known ocean dynamics and available LGM proxy records, given their uncertainties?

2. What magnitude of modern to LGM changes in oceanic and atmospheric state are required to obtain a consistent LGM Atlantic Ocean state? Are the required changes 
uniform, or are regional patterns evident?

3. What Atlantic meridional overturning circulation state or states are consistent with available LGM deep ocean proxy data? More generally, what is required to constrain deep ocean water mass distributions and pathways with sparse deep ocean tracer data?

This chapter provides background and context for the rest of the thesis. Section 1.1 provides a more extended motivation for reconstructing LGM Atlantic Ocean conditions. Section 1.2 then describes limitations of common approaches to studying the LGM climate, and argues that synthesizing models and data through dynamical reconstruction is an important, but infrequently used, approach. The dynamical reconstruction approach applied in this thesis is introduced, and previous applications of the technique are briefly reviewed to provide evidence for the suitability of the approach. The chapter concludes with a preview of the contents of the thesis.

\subsection{The Last Glacial Maximum}

Humankind is undertaking an unprecedented experiment by releasing massive amounts of $\mathrm{CO}_{2}$ and other greenhouse gases into the modern atmosphere - the anthropogenic climate experiment. While it is well accepted that serious shifts in climate are a potential, and even likely, outcome of this experiment, prediction of future climate change is a difficult exercise with many uncertainties [Solomon et al., 2007]. Climate models are the primary tool used to project anthropogenic climate change, but estimating how well such models simulate climates different from today's is a challenge [Braconnot et al., 2012].

Fortunately, there is a source of data on how our planet responds under a variety of climate conditions: Earth's geologic record includes clear evidence of past climates that were very different from today's. Characteristics of past climates are inferred through proxy records - preserved chemical, isotopic, or physical characteristics that can be used to estimate past conditions. The mapping from preserved properties to past climate charac- 
teristics relies on modern calibrations - demonstrations that modern climate characteristics are preserved in a consistent way in modern sediments.

A number of characteristics make the Last Glacial Maximum an excellent case study for dynamical reconstruction of a past climate.

- The LGM is a period of relative climate stability, apparently without large shifts in land ice, sea level, or atmospheric $\mathrm{CO}_{2}$ concentrations [Clark et al., 2009, Schmitt et al., 2012]. This stability is key to interpretation of proxy records, and greatly simplifies application of climate models to the period (e.g. Braconnot et al. 2007a).

- Amongst relatively stable episodes of the last 100,000 years, the LGM is the most drastically different climate from today's. Given uncertainties in proxy records of climate, large changes are much easier to identify and quantify (e.g. Adkins et al. 2002).

- Atmospheric $\mathrm{CO}_{2}$ concentrations at the LGM were lower by about 90 ppmv than at the pre-industrial [Schmitt et al., 2012], a difference that appears to have been important to sustaining colder LGM conditions [Shakun et al., 2012]. This period thus provides an important case study for the role of $\mathrm{CO}_{2}$ in climate [Schmittner et al., 2011]).

- Continental configurations were very similar to today's [Peltier, 2004], simplifying attribution of climate changes.

- The LGM is one of the most extensively studied periods in Earth's history, so that relatively large numbers of proxy records are available. Uncertainties in proxies themselves, and in the dating of the proxy records, are generally lower for the LGM than for periods further back in time.

- Substantial effort has been made to build large, quality-controlled compilations of proxy records (e.g. CLIMAP Project Members 1976, Marchitto and Broecker 2006, Waelbroeck et al. 2009, Marchal and Curry 2008). These compilations greatly simplifying the task of using proxy records. 
It is now apparent why the LGM is appropriate for dynamical reconstruction, and why the results are important to current climate concerns. It is, at this point, less obvious why it is sensible to focus on the LGM Atlantic Ocean state. The Atlantic Ocean is one of the best studied regions for the LGM (see Chapter 2), and is also a relatively well-understood part of the modern climate (see Chapter 3). The Atlantic is also dynamically important as a deep water mass formation region. Finally, significant shifts in Atlantic Ocean state have been proposed to have occurred between the LGM and today (e.g. Lynch-Stieglitz et al. 1999b, Curry and Oppo 2005), providing a number of intriguing hypotheses to test.

\subsection{Dynamical reconstruction}

Most studies of LGM ocean circulation focus on (1) proxy records, which are often interpreted with the aid of qualitative ideas about shifts in ocean circulation; or (2) dynamical models of LGM changes (e.g. theories, idealized models, coupled climate models), which are sometimes compared in a qualitative sense with selected proxy records. These approaches have contributed much to our understanding of LGM climate (Chapter 2 presents a full review), but there are risks in focusing too heavily on one approach. The qualitative ideas of ocean circulation sometimes used to interpret proxy records may be physically unrealistic, and the state obtained in dynamical models can be quite far from observations, making it difficult to apply inferences made from the models.

The need to move from qualitative to quantitative model-data comparisons has recently become a focus for both the LGM modeling community [Braconnot et al., 2012] and the LGM proxy community ${ }^{1}$. There is also increasing recognition from the broader modeling community of the potential of paleo model/data comparisons to improve model forecasts of future climate [Schmidt, 2010].

Dynamical reconstruction moves beyond quantitative comparison to build estimates of

\footnotetext{
${ }^{1}$ The Comparing Ocean Models with Paleo-Archives (COMPARE) workshop, held in March 2012 in Bremen, Germany, focused on the topic of model/data comparisons and approaches to synthesizing models and data.
} 
climate that are consistent with both model and data. The exercise of building a model-data synthesis brings to the investigator a number of advantages. First, serious consideration can be given to the uncertainties and biases of both models and data, providing a more holistic view of the problem at hand. Second, a quantitative measure for "success" is defined a priori, with explicit weighting of the relative importance of diverse data constraints. This approach permits formal statistical hypothesis testing.

Quantitative model-data synthesis has been previously applied to the LGM Atlantic (see Section 2.4.2 for a thorough review). For this thesis, the ocean state is estimated using the method of Lagrange multipliers, whereby a least squares fit of a dynamical model to the proxy data is computed using an algorithm based on the model adjoint [Wunsch and Heimbach, 2007]. In this approach, the model-data misfit is reduced by computing improvements to uncertain initial and boundary conditions (the control variables). Quantitative uncertainties assigned to all proxy records and to the control variables play as much a part in the solution obtained as do the data themselves. Section 3.2 provides a more complete overview of the state estimation methodology used in the thesis. For now we simply note that, as compared to previous model-data syntheses for the LGM Atlantic, this thesis uses a more complete dynamical model and a larger number and greater diversity of proxy records.

The tool applied in this thesis to build dynamical reconstructions is the Massachusetts Institute of Technology General Circulation Model (MIT GCM) [Marshall et al., 1997, Adcroft et al., 2004] and its adjoint [Heimbach et al., 2005]. Estimates of ocean circulation and properties developed using this tool in combination with data are called ocean state estimates and the process of making the model-data synthesis is called ocean state estimation. The MIT GCM and its adjoint have been applied to develop a variety of modern ocean state estimates. A particularly substantial modern effort is the Estimating the Circulation and Climate of the Ocean (ECCO) project, a large, multi-institution project that has focused primarily on estimating the global ocean circulation [Stammer et al., 2002, Wunsch and Heimbach, 2007, 2012, in press]; vast numbers of observations have been successfully 
incorporated in these state estimates, including, for example, hydrography, satellite observations of sea surface temperature and sea surface height, autonomous float data, and data from bottom pressure recorders. Examples of regional ocean state estimates include the Eastern Atlantic Ocean [Gebbie et al., 2006], the Southern Ocean [Mazloff et al., 2010], and the Labrador Sea [Fenty, 2010, Fenty and Heimbach, 2012]. These estimates have incorporated a wide diversity of observational data, and each estimate has proven useful in understanding new aspects of the modern ocean circulation.

Due to the poor dating resolution of sediment cores from the LGM period, and the sparsity of LGM proxy observations, LGM proxy records are typically assumed to represent average conditions over thousands of years; for some proxies, it is possible to estimate conditions in specific seasons (e.g. summer or winter), but these estimates still represent a very long term mean of summer or winter conditions over the LGM period. Due to these limitations in the timescales that can be reconstructed with available data, a seasonal steady state assumption is adopted here - that the LGM Atlantic can be adequately represented with a seasonally varying, but otherwise stable in time, steady state (a seasonal steady state). Previous LGM model-data syntheses have generally made a direct steady state assumption (no seasonal cycle), and have built this assumption into the model framework (see Section 2.4.2).

Here, instead of building a steady state assumption into the model, an alternate approach is taken: to search for an ocean state estimate that satisfies the seasonal steady state assumption. A new type of ocean state estimate is defined: an equilibrium ocean estimate $(\mathrm{EOE})$ is an estimate of ocean state that is

1. consistent with known dynamics,

2. consistent with available data given their uncertainties, and

3. consistent with a steady state assumption (whether fully steady or cyclic in nature).

It is likely that there are a number of different approaches that could be used to achieve these three goals. In this thesis, the philosophy used by the paleo climate modeling community is adopted (see Section 2.3.1): that a long-running model simulation with limited 
drift is indicative of a quasi-steady (or quasi-equilibrium) circulation. Long-running model simulations have another advantage: interior ocean properties are set largely by the atmospheric forcing applied to the model, rather than the initial properties from which the model was started. For a problem such as reconstructing the LGM ocean state, a full dynamical connection between atmospheric forcing and interior ocean conditions. Without such a connection, one can not investigate, for example, what deep interior ocean data imply about surface boundary conditions.question that has arisen

\subsection{Terminology}

In drawing upon proxy evidence of past climates, modern observations, forward models, and inverse modeling techniques, this thesis necessarily relies on much terminology, the exact meaning of which may sometimes be unclear. To aid the reader, a list of acronyms used in the thesis is provided on page 19 and the following paragraphs describe how key terminology is used in the thesis.

The Last Glacial Maximum. Exact definitions of the LGM time period vary. The estimates of LGM state developed in this thesis rely on proxy record compilations that either define the LGM to span 19-23,000 years ago [Waelbroeck et al., 2009] or 18-21,000 years ago [Marchal and Curry, 2008]. The first definition is followed here - that the period under study spans 19-23,000 calendar years before present (19-23 kyr bp).

Proxy record. In paleoclimate literature, the word "record" often refers to measurements made on a sequence of samples from, for example, a sediment or ice core; in the standard terminology, a proxy record is a time-varying sequence of data corresponding to a particular section of sediment or ice core material. In this thesis, only the LGM time period is discussed, and all compilations of proxy evidence used in the thesis have (1) assumed the LGM spans a given range of years, (2) computed an average of available sediment core evidence over that period, and (3) have reported only the average conditions over the LGM period. Therefore, for the purposes of this thesis, a proxy record (or simply record) is a 
single estimate of LGM conditions at a given geographic location.

Near sea surface temperature. Sea surface temperature (SST) can mean different things to different communities. Those who estimate SST from satellites are truly measuring the skin temperature at the surface of the ocean. In conditions of strong thermal stratification (i.e. in summer), the skin temperature may apply over only the top few millimeters. Likewise, meteorologists and atmospheric dynamicists may be interested in SST only as a boundary condition on atmospheric dynamics; this boundary condition is the skin temperature of the surface ocean. The term SST is avoided in this thesis and near sea surface temperature (NSST) is used instead to remind the reader that we are not discussing the skin temperature. In the work presented herein on modern conditions (Chapter 3), NSST is taken to be the temperature of the upper most cell of the model, which is $10 \mathrm{~m}$ thick. In the work presented on LGM conditions (Chapters 4 and 5), NSST is taken to be the mean temperature of the top three cells of the model, a depth of $30 \mathrm{~m}$ in total. NSST reconstructions for the LGM are based on organisms that inhabit a variety of depths in the water column; $30 \mathrm{~m}$ is taken as a conservative estimate of the potential habitats of these organisms.

\subsection{Structure of the thesis}

In this thesis, I argue that LGM ocean conditions can be best understood through dynamical reconstruction. It could be argued that the circulation estimates presented in this thesis are the most complete dynamical reconstructions of the LGM Atlantic Ocean currently available. The thesis presents a number of contributions to understanding of the LGM Atlantic that would not have been possible without a dynamical reconstruction. Despite the power of dynamical reconstruction, incorporating data and models in a single estimate or reconstruction is a challenge: it requires honest consideration of the limitations and potential biases of both models and data (which are often poorly known), and the technical machinery involved is difficult to learn and computationally expensive to apply. The contributions and remaining challenges of this work are described in the remainder of the thesis, which 
is structured as follows.

Chapter 2 surveys current knowledge of the LGM Atlantic, with detailed discussions of proxy evidence, numerical modeling studies, and previous approaches to dynamical reconstruction. A particular contribution of this chapter is an evaluation of the relative ease with which available proxy types could be incorporated in a model-data synthesis.

In Chapter 3, a framework for ocean state estimation that is appropriate to study of paleo circulation is presented. The utility of this framework is explored in the context of the modern ocean circulation - a system that is much better observed and understood than the LGM ocean. An approach to modeling $\delta^{13} \mathrm{C}_{\mathrm{DIC}}$ and $\delta^{18} \mathrm{O}_{\text {water }}$ is presented and tested in state estimates constrained by modern water column measurements of $\delta^{13} \mathrm{C}_{\mathrm{DIC}}$ and $\delta^{18} \mathrm{O}_{\text {water }}$. Two techniques for extending the length of ocean state estimates, asynchronous timestepping and carry-over control, are described and shown to be appropriate and effective. Significant limitations of the state estimation framework are identified.

Estimation of the LGM upper ocean state is the focus of Chapter 4. Four diverse near sea surface temperature (NSST) proxy compilations are used to constrain the estimates. The chapter explores disagreement amongst the datasets, the extent to which the LGM NSST data require an atmospheric state significantly different from today's, and the consistency of the state estimates with withheld data. Results are compared to existing coupled model simulations of the LGM climate.

Chapter 5 is more exploratory in nature. State estimates are presented that are constrained by deep ocean proxy records (benthic $\delta^{13} \mathrm{C}_{\text {calcite }}$ and $\delta^{18} \mathrm{O}_{\text {calcite }}$ data, see Chapter 2 for details on these proxies) in addition to the NSST data used in Chapter 4. The consistency of these data with a meridional overturning circulation different from today's is explored.

Finally, Chapter 6 concludes the thesis. A vision for the future of ocean state estimation is presented, along with a discussion of what is required to enable state estimation for scenarios in which a steady state assumption is required. Novel contributions of the thesis are summarized, as are limitations. Promising directions for future progress are presented. 


\section{Chapter 2}

\section{Last Glacial Maximum Atlantic}

This chapter summarizes what is currently known or hypothesized about the state of the Last Glacial Maximum (LGM) climate, and particularly about the state of the LGM Atlantic Ocean. Due to the breadth and depth of previous work focused on the LGM, this review cannot be comprehensive. Instead, the focus is on previous work that is particularly important as background for the rest of thesis. The chapter begins with an overview of the LGM climate in Section 2.1. Section 2.2 describes proxies for upper ocean and deep ocean state at the LGM, and includes a detailed discussion of potential sources of uncertainty and bias in reconstructions of climate with these proxies. Section 2.3 summarizes results obtained with traditional forward models of the LGM. Efforts to seriously consider models and data together are described in Section 2.4, which covers previous work in quantitative model-data comparison as well as in model-data synthesis. Section 2.5 describes which datasets were chosen as data constraints in the thesis and why, and Section 2.6 concludes the chapter.

In reading this chapter (and indeed the whole thesis), it is important to keep in mind that observations or modeling results from a specific geographic region cannot be used to make global inferences. Indeed, the global climate system is connected on a multitude of spatial and temporal scales, so that changes in climate in one region may be associated with changes of an opposing sign in another region (e.g., shifts in the westerlies would 
cause increased wind speed in some regions and decreased wind speed in others). Even climate characteristics for which the modern-LGM changes could be predominantly of one sign (e.g., LGM air temperatures were generally colder than today's), the magnitude of change can have strong regional dependencies.

\subsection{Geography and atmospheric State}

Physical geography. The location of the land masses at the LGM was very similar to that of today, though large terrestrial ice sheets and the associated sea level lowering caused substantial changes in Earth's geography. The timing of the LGM sea level lowstand appears to be in good agreement with the timing of the maximum extent of ice sheets and mountain glaciers; the peak LGM glaciation occurred from 26.5 to $19 \mathrm{ka}$, and global ice volume (and therefore sea level) was relatively stable during this period [Clark et al., 2009]. Long term sea level records from Barbados [Fairbanks, 1989], the Sunda Shelf of Indonesia [Hanebuth et al., 2000], and the J. Bonaparte Gulf region of northern Australia [Yokoyama et al., 2000] are consistent with a globally averaged sea level change from today to the LGM of about $125 \mathrm{~m}$. Interpretation of local sea level changes are complicated by isostatic adjustment the dynamic adjustment of the asthenosphere to accommodate the mass of the lithosphere. Ice sheet models that include the effect of isostatic adjustment can be used to infer global sea level changes from relative sea level records; this approach was used by Peltier [2004] to estimate an LGM sea level lowering of $125 \mathrm{~m}$ and by Clark et al. [2009] to estimate a lowering of $130 \mathrm{~m}$.

Due to lowered sea levels, several key changes in ocean bathymetry occurred that affected the Atlantic Ocean: the Bering Strait was closed, shallow continental shelves (such as that off the east coast of North America) were exposed, Hudson Bay was exposed, and all sills were shallower by about $125 \mathrm{~m}$ (see Figure 2-1). The storage of freshwater in terrestrial ice sheets also resulted in an increase in mean ocean salinity of about 1.1 relative to today [Adkins and Schrag, 2001]. Due to the nonlinear dependence of density on tempera- 


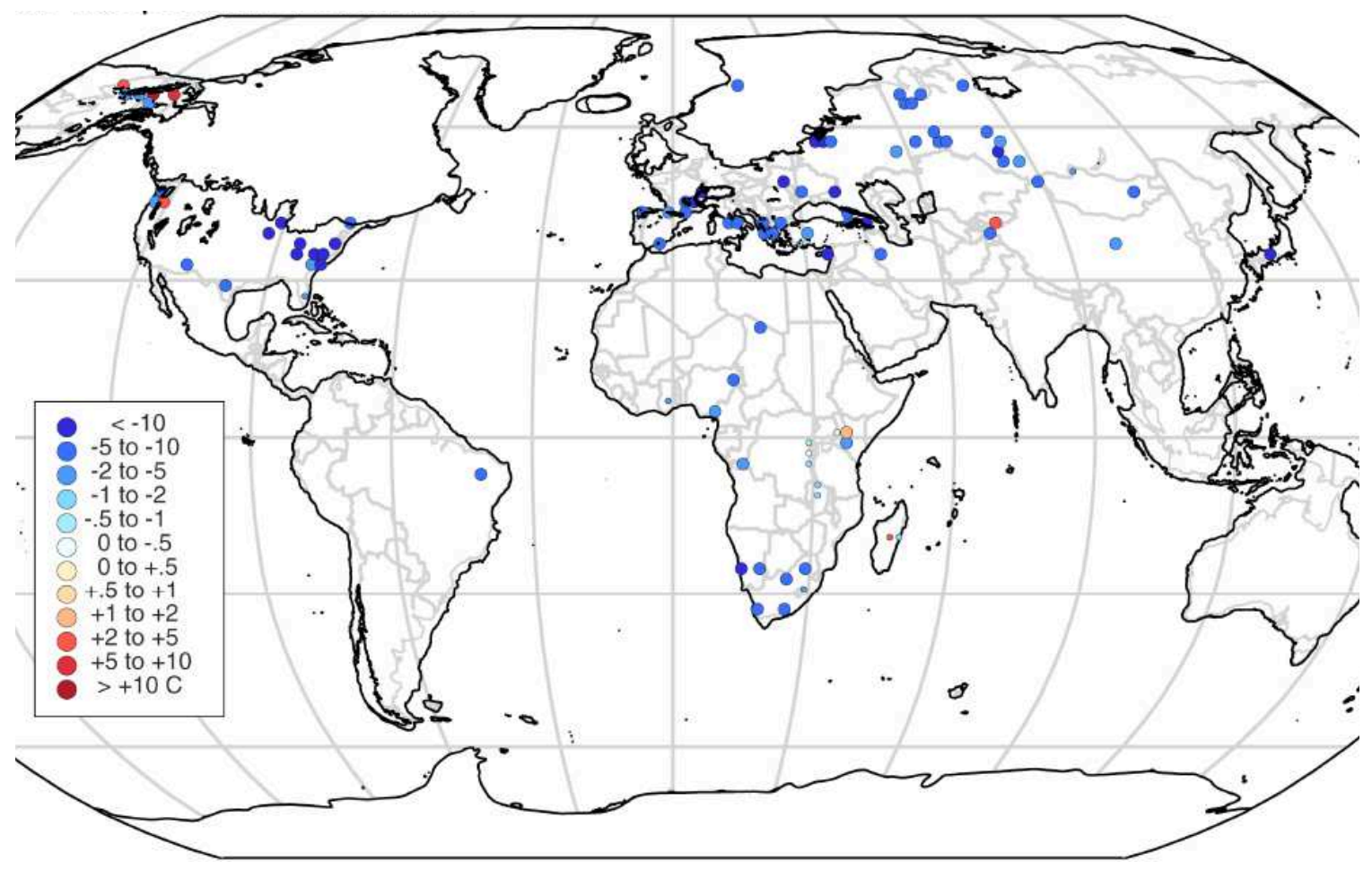

Figure 2-1: Overview of LGM ice sheets (solid white regions), coastlines (overlaid in black on modern map in gray), and terrestrial temperature anomalies in ${ }^{\circ} \mathrm{C}$ from pollen and macrofossil data (colored circles, size indicates significance). Ice sheets and coastlines are a blended combination of three recent ice sheet reconstructions. Cropped version of figure from Braconnot et al. [2012]; see references therein for data sources.
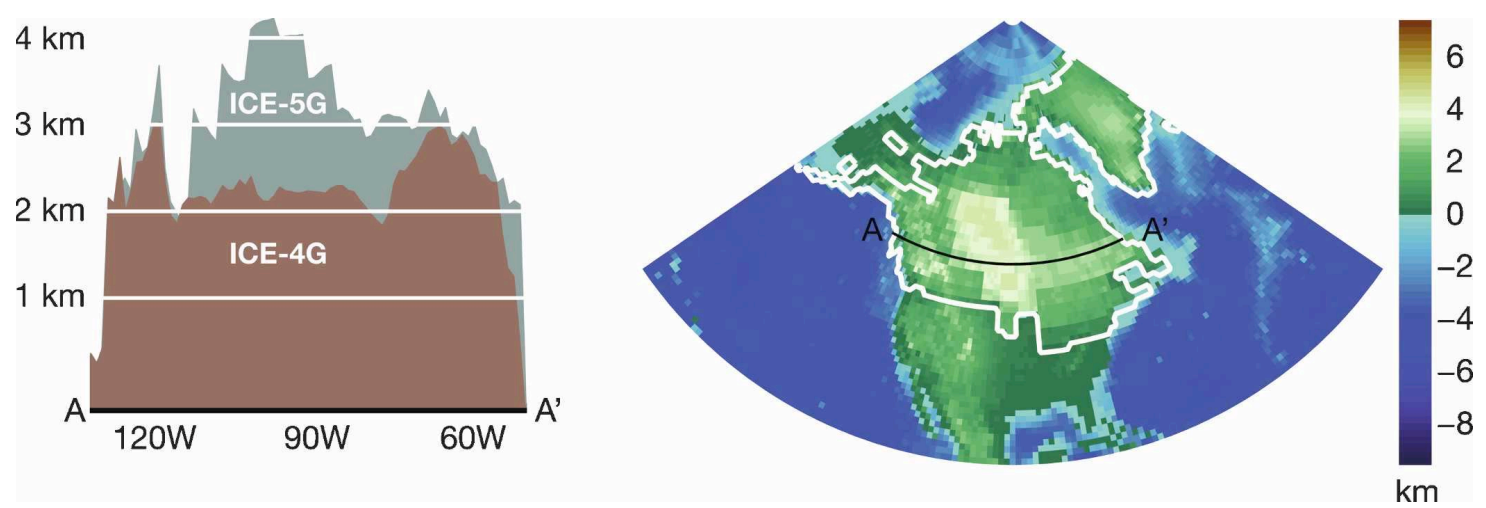

Figure 2-2: Comparison of ICE-4G and ICE-5G elevation across the Laurentide ice sheet at $55^{\circ} \mathrm{N}$ (left). On the right, ice sheet extent as reconstructed by ICE-5G is outlined in white, and the $55^{\circ} \mathrm{N}$ section is marked in black. Figure from Li and Battisti [2008]. 
ture and salinity, the increase in mean salinity at the LGM implies that salinity likely played a more significant role in controlling the LGM density field than it does today [Adkins and Schrag, 2001].

LGM global mean sea level is relatively well-constrained and permits direct estimation of LGM global glacial ice volume. Ice sheet topography is much more difficult to estimate, but is important to constrain as it impacts many aspects of atmospheric state including the wind field. The Environmental Processes of the Ice Age: Land, Oceans, Glaciers (EPILOG) project was an effort to reconstruct the state of the Earth in glacial states; a special issue collection of EPILOG papers [Clark and Mix, 2002] focused on records of ice sheet extent, sea level change records, models of ice sheets and glacial isostatic adjustment, and geochemical ice volume proxies. Siegert [2001] provides an extensive overview of observations and models of ice sheets in the Late Quaternary. Siegert [2001] notes that, although physical indicators such as glacial moraines and glacial scour provide a relatively accurate picture of ice sheet extent, ice sheet thickness is much more difficult to estimate from surficial geologic evidence. Relative sea level records collected worldwide have been used in concert with an ice sheet model and a model of isostatic adjustment to infer the mass of individual LGM ice sheets [Peltier, 2004]. The Peltier [2004] ICE-5G gridded estimate of LGM ice sheet topography, coastlines, and ocean bathymetry has proven useful to LGM modeling efforts (e.g., Braconnot et al. 2007a). In using these estimates it must be remembered that LGM ice sheet topography remains uncertain (see Figure 2-2).

The majority of available dust records show glacial periods to have been generally dustier and interglacials to have been more vegetated [Rea, 1994, Yung et al., 1996, Hesse and McTainsh, 1999]. Greenland and Antarctic ice core records show a 2 to 20 fold increase in dust deposition rates at the LGM as compared with the present while low and mid-latitude marine sediment core records show up to a 5 fold increase [Kohfeld and Harrison, 2001]. In the Kohfeld and Harrison [2001] compilation of data from ice cores and marine sediment cores, nearly all sites show increases in dust deposition at the LGM compared to today. It is commonly thought that increased dust fluxes at the LGM are at least 
partially caused by drier terrestrial conditions with significant changes in vegetation, at least in some regions [Hesse and McTainsh, 1999, Kohfeld and Harrison, 2001]. The Andean region is an exception: records indicate that the region is actually drier during interglacials than during glacials [Rea, 1994]. A global compilation of lake level reconstructions (see supplementary material of Braconnot et al. 2012 indicates that LGM conditions may have been drier than today in the tropics, but wetter than today in the low-mid latitudes, a scenario supported by the PMIP2 models [Boos, 2012].

Atmospheric state. Reconstructing the atmospheric state at the LGM with proxy observations is a major challenge. Mahowald et al. [1999] compare a model of the dust cycle at the LGM with proxy records and find evidence for a reduced hydrologic cycle at the LGM. As shown in Figure 2-1, pollen and macrofossil based proxies indicate LGM temperatures over land were typically $5-10^{\circ} \mathrm{C}$ colder than modern with even larger changes in the Southeastern United States. The intense LGM cooling in this region and the relatively warm conditions in Alaska are both consistent with the generally accepted notion that the Laurentide ice sheet caused a substantial shift in atmospheric circulation (see below).

It is sometimes assumed that high atmospheric dust concentrations (and therefore high depositional fluxes) indicate high wind speed. In fact, this assumption is not supported by observations [Rea, 1994] nor by the physics of sediment transport. Instead, in the modern climate, high atmospheric dust concentrations have been linked to low rainfall in source regions [N'Tchayi et al., 1994, Ekstrom et al., 2004]. Dust flux rates can also be linked to rainfall rates in the deposition area since dust deposition is higher during rainfall [Kohfeld and Harrison, 2001].

While dust concentrations cannot be used as a proxy for wind speed, grain size distributions have been used for this purpose [Rea, 1994, Parkin and Padgham, 1975]. Clemens [1998] did a careful study of modern eolian deposition in the Arabian Sea to compare characteristics of the dust with measured characteristics of the wind field; eleven sediment traps were used over a full annual cycle and compared against meteorological data on wind speed 
and barometric pressure. A high correlation of eolian grain size with barometric pressure $(\mathrm{r}=-0.91)$, and with wind speed $(\mathrm{r}=0.84)$ was found. Other researchers have linked the quantity of magnetite in aeolian dust to transport processes such as wind speed [Maier and Scholger, 2004] based on the wind power required to deflate heavier magnetite particles from source regions and transport them. Magnetite quantity is a convenient measure for application to paleo-environments as magnetic susceptibility is relatively straightforward to measure. Some information about wind direction can be inferred by matching characteristics of deposited dust with characteristics of source regions. This approach of identifying dust "fingerprints" requires that the measured dust properties be part of the dust or be transported with the dust, be distinctive of the source region, and be conservative during transport [Grousset and Biscaye, 2005]. Biological tracers such as opal phytoliths, freshwater diatoms, and pollen can also be used to constrain the latitude of a dust source region. The most promising tracers for better constraining dust sources appear to be radiogenic isotopes such as strontium, neodymium, and lead [Grousset and Biscaye, 2005]

In a survey of many available proxy records of the dust cycle, Kohfeld and Harrison [2001] conclude that dust records from Greenland and Antarctic ice cores do not indicate significant changes in the LGM high latitude atmospheric circulation relative to today; Hesse and McTainsh [1999] agree, finding that increased dust fluxes to Antarctica at the LGM are due to increased source region aridity at the LGM, rather than to windier conditions. In contrast, LGM proxy records are used by Yung et al. [1996] to infer an increased wind field in both hemispheres and by Delmonte et al. [2004] to infer a southward shift of the southern hemisphere polar vortex between 20 to 10 kya. Parkin and Padgham [1975] infer changes in the trade winds at the LGM based on observed changes in grain size. Florineth and Schluchter [2000] combine geologic mapping of European alpine glacier locations through time with other terrestrial records such as lake levels; they infer that the polar front may have been centered near $44^{\circ} \mathrm{N}$ at the LGM, leading to a southerly atmospheric circulation (the modern circulation is predominantly westerly) and lower precipitation in central and northern Europe at the LGM relative to today. 
Given the relative paucity of proxy records of atmospheric state, climate models have been heavily applied to estimate LGM conditions. Coupled models of the LGM climate will be discussed in Section 2.3.1; in this section we note a few model results that are specific to understanding LGM atmospheric conditions. The effect of the large terrestrial ice sheets on atmospheric stationary waves is relatively well understood [Cook and Held, 1992, Jackson, 2000, Roe and Lindzen, 2001]; with regard to the Laurentide ice sheet, the Jet Stream was likely shifted northward to the west of the ice sheet (thus explaining the warm Alaskan temperatures seen in Figure 2-1) and was pushed south to flow around the southern boundary of the ice sheet over the continental United States (thus explaining cold LGM temperatures in the Southeastern United States in Figure 2-1). Li and Battisti [2008] investigate atmospheric dynamics in a single coupled model simulation of the LGM (the Community Climate System Model, version 3 - CCSM3) and find stronger, more zonally oriented atmospheric jets over the Atlantic Ocean as compared to today. Li and Battisti [2008] also find that transient eddy activity is weaker over the Atlantic Ocean in the LGM simulation than in the modern one, implying reduced storminess at the LGM.

Rojas et al. [2009] analyze a variety of coupled model simulations of the LGM to investigate changes in the Westerlies in the Southern Ocean. These authors find that while surface equator to pole temperature gradients were larger in the LGM models than in the modern climate, the tropospheric temperature gradients are actually reduced for the LGM simulations; decreased winds near the surface and in the upper troposphere result [Rojas et al., 2009]. Toggweiler et al. [2006] propose that significant shifts occurred in the latitudinal position of the westerlies in the Southern Hemisphere between the LGM and today and that these shifts played a major role in deep ocean circulation differences between the LGM and today. They review a few proxy-based studies indicating that the Southern Hemisphere westerlies could have been as much as $7-10^{\circ}$ further north at the LGM than today; they also review observational evidence that the modern westerlies are slowly shifting poleward as a result of global warming. 


\subsection{Proxies of LGM ocean state}

The LGM is a heavily studied period in the geologic record, and many proxies that cannot be used for older periods work well for the LGM (e.g., radiocarbon). A major challenge in designing a dynamical reconstruction of LGM ocean state is to decide which proxy types, and which specific proxy records, are most appropriate for inclusion in a modeldata synthesis. This section describes a variety of proxies of LGM ocean state. For each proxy type, an evaluation is made of how appropriate the data are for quantitative modeldata comparisons or syntheses with that proxy type. Note that the fundamental issues are essentially the same for either model-data comparisons or syntheses, and the evaluations herein are meant to apply to either. Note that, although not considered in the evaluations of this chapter, many more practical issues arise in practice in building a model-data synthesis than in quantitative model-data comparisons.

The following conditions are key to meaningful model-data comparisons or syntheses: a mapping exists between properties recorded by the proxy and properties that can be incorporated in existing ocean models, quantitative uncertainty assignments can be made for the data, sufficient numbers and distribution of records exists to warrant a comparison or synthesis effort, and the data must be publicly available or otherwise accessible to the investigator. These evaluations represent a personal scientific judgement, and others will certainly disagree with some aspects of the evaluations. Nonetheless, it is hoped that these evaluations will aid other investigators who are interested in quantitative analysis of models and data together.

This section is organized as follows. Section 2.2.1 provides a general discussion of potential uncertainties and biases in marine proxies; uncertainties play in model-data synthesis, and thus must be carefully considered. Sections 2.2.2 and 2.2.3 then describe selected proxies for upper and deep ocean conditions, respectively. As a preview of what is to come in the rest of the thesis, Table 2.1 lists the proxies used in later chapters of the thesis to constrain our estimates of LGM ocean state; these proxies are more thoroughly reviewed here than proxies that have not been included in the state estimates. 


\begin{tabular}{|l|l|l|l|}
\hline Source & Parameter & \# records & Reference \\
\hline Foraminiferal assemblage & SST & 174 & Kucera et al. [2005b] \\
Dinoflagellate cyst assemblage & SST & 52 & De Vernal et al. [2005] \\
Alkenones & SST & 54 & Rosell-Melé et al. [2004] \\
$\mathrm{Mg} / \mathrm{Ca}$ & $\mathrm{SST}$ & 33 & Barker et al. [2005] \\
\hline Benthic foraminifera & $\delta^{18} \mathrm{O}_{\text {calcite }}$ & 169 & Marchal and Curry [2008] \\
Benthic foraminifera & $\delta^{13} \mathrm{C}_{\text {calcite }}$ & 147 & Marchal and Curry [2008] \\
\hline
\end{tabular}

Table 2.1: Summary of proxies included in LGM state estimates presented in the thesis. The number of records that fall in the model domain is noted for each proxy type. The first four/last two proxies are used to infer upper/deep ocean conditions. A total of 629 proxy records are incorporated in the LGM state estimates.

\subsubsection{Introduction to potential uncertainties and biases}

As will be clear in Sections 2.2.2 and 2.2.3, each marine proxy is affected by a distinct combination of uncertainties and biases, so that each has unique advantages and disadvantages. This section provides an overview of potential pitfalls affecting interpretation of marine proxies. The section begins with a list of general issues, a summary that will then be made more concrete with a case study of uncertainties and biases for $\delta^{18} \mathrm{O}$ as a proxy.

Instrumental precision in the measurement of the proxy itself is often quite small and can be misleading if quoted in isolation. Care must be taken to incorporate other, often larger, uncertainties associated with proxy interpretation. Some of these are as follows.

- Inter-laboratory differences in instrument calibration or in preparation techniques can lead to biases between samples prepared in different laboratories (see, e.g., Ostermann and Curry 2000).

- Although proxies are typically assumed to represent either seasonal, annual, or longterm mean climate properties, proxies may carry a strong signal of more occasional but strong events. Examples include spring bloom events in the high-latitudes that can affect inferences of annual mean temperature from planktonic proxies (see, e.g., Dale 2001), and submarine landslides that complicate interpretation of grain size records (see, e.g., McCave and Hall 2006). 
- Proxies are typically converted into a single climate variable through techniques such as correlation of modern measurements with modern climate properties. In reality, most proxies correspond to multiple environmental variables (see, e.g., Morey et al. 2005). The assumption that a single climate characteristic dominates the proxy's variation back in time leads to uncertainties that are difficult to quantify.

- Several issues are largely specific to planktonic proxies. First, what plankton record in their own habitat may not match what is preserved in a sediment core; for example, biases can occur due to the impact of dissolution (see, e.g., Barker et al. 2005) or diagenetic alteration (chemical changes that occur within the sediment after deposition on the seafloor). Second, the representativeness of a given organism to a specific depth range of the water column may be poorly constrained (see, e.g., Farmer et al. 2007).

- For proxies that record conditions at the seafloor, there is a mismatch between what is recorded by the proxy and what can be adequately represented in today's climate models. Benthic organisms live on or in the sediment, an area that is in a boundary layer with a very different dynamical regime than the rest of the water column. With current computational capabilities, climate models are run at resolutions far too low to accurately capture boundary layer physics. Just as an example, it is not uncommon to have vertical jumps of $500 \mathrm{~m}$ in elevation between adjacent grid cells in deep layers of ocean models (see, e.g., Forget 2010).

To make the discussion of potential uncertainties more concrete, uncertainties associated with $\delta^{18} \mathrm{O}_{\text {calcite }}$ as a proxy are now used as a case study. This proxy is chosen because there are particularly extensive uncertainty analyses available in the literature. A particularly valuable resource is a thorough review in Waelbroeck et al. [2005] of uncertainties in measurements of $\delta^{18} \mathrm{O}_{\text {calcite }}$ in planktonic foraminifera from Holocene sediment samples. Unless otherwise noted, the following discussion is based on Waelbroeck et al. [2005].

Although standard deviations of $0.05-0.06 \%$ can be obtained in repeated measurements of a single sample of $\delta^{18} \mathrm{O}_{\text {calcite }}$ on a single mass spectrometer, larger errors and/or biases 
can be introduced with small sample sizes or when the sample's isotopic composition is far from that of the reference standard. A correction of this last bias has been developed [Ostermann and Curry, 2000] and is applied to new measurements in most laboratories; unfortunately, it is sometimes unclear whether the correction has been applied to a given published record, particularly for old measurements. In addition, differences in cleaning and sample preparation techniques can introduce an uncertainty on the order of $0.2 \%$. Measured $\delta^{18} \mathrm{O}$ values have sometimes been found to depend on fossil foraminifera size, and this dependency can vary across species, size fractions, and regions.

Measurements of the $\delta^{18} \mathrm{O}$ on individual foraminifera of a given species in a specific depth or time interval will show a range of $\delta^{18} \mathrm{O}$ values. This intraspecies variability is due to the recording of seasonal and short-term water column variability (most shallowdwelling planktonic foraminifera have a 2-4 week lifecycle, Schiebel and Hemleben 2005); the influence of bioturbation and the co-occurrence of several genotypes of a morphospecies can also introduce variability. For a single interval near sediment core tops, the $\delta^{18} \mathrm{O}$ range observed amongst individual shells is $\approx 1.2-1.5 \%$ for planktonic species such as Orbulina universa, Globigerinoides sacculifer, and pink and white G. Ruber and can exceed $2 \%$ for thermocline dwelling species such as Neogloboquadrina dutertrei and Pulleniatina obliquiloculata. For a subset of data and assumed instrument precision of $0.06 \%$, Waelbroeck et al. [2005] estimate that use of 20 shells implies a precision of 0.09-0.19\% for planktonic foraminifera. Due to much lower variability in water properties at depth, precision for benthic species is significantly better. Post-depositional processes can also alter the observed $\delta^{18} \mathrm{O}_{\text {calcite }}$ signal: bioturbation may act to dampen the Holocene to LGM signal, and partial post-depositional dissolution may alter the $\delta^{18} \mathrm{O}_{\text {calcite }}$ of the foraminifera. Uncertainties associated with these processes are not well-constrained.

Records of $\delta^{18} \mathrm{O}_{\text {calcite }}$ tend to over-represent conditions during peak growing seasons and peak years, an issue that varies amongst genera. As an example, Mulitza et al. [2003] find that for a station off Mauritania at Cape Blanc, G. ruber and G. sacculifer seem to record summertime SSTs, O. universa seems to record annual mean conditions, and $G$. 
bulloides and $G$. inflata record conditions more similar to winter conditions. Another issue of concern is that many genera can be found in a wide range of depths, calling into question their use as proxies for sea surface conditions. However, Mulitza et al. [2003] show that at an eastern equatorial Atlantic station, measured $\delta^{18} \mathrm{O}_{\text {calcite }}$ is in line with expected values very near the surface, implying that although they observed foraminifera living at a wide range of depths, the calcification depth for the foraminifera genera they studied was likely in the surface mixed layer.

Although proxies are typically used to reconstruct a primary environmental variable, they are often impacted by multiple climate conditions. For example, a unit change in $\mathrm{pH}$ has an approximately $1 \%$ effect on $\delta^{18} \mathrm{O}_{\text {calcite }}$ [Zeebe, 1999]; if surface ocean $\mathrm{pH}$ was significantly higher at the LGM than today, as claimed by Sanyal et al. [1995], this effect could introduce a significant biases.

Quantifying all potential uncertainties and biases and using them to compute an overall uncertainty is a formidable challenge. Existing compilations with overall uncertainty estimates include the Waelbroeck et al. [2005] compilation of 2100 measurements of Holocene planktonic foraminifera $\delta^{18} \mathrm{O}_{\text {calcite }}$ and the Marchal and Curry [2008] compilation of 198 Holocene and $180 \mathrm{LGM}$ measurements of $\delta^{18} \mathrm{O}_{\text {calcite }}$ on benthic foraminifera. Each of these studies assigned a uniform uncertainty of $0.2 \%$ to all $\delta^{18} \mathrm{O}_{\text {calcite }}$ records.

\subsubsection{Upper ocean state}

The surface ocean is the connection between the atmosphere and the rest of the ocean: it serves as a key boundary condition on atmospheric dynamics, and is the region where nearly all deep ocean properties originate. The portion of the upper ocean that receives solar insolation, the euphotic zone, is also the most productive zone in the ocean. Fortunately, characteristics of the upper ocean from the past are relatively well-preserved in sediment cores. A number of proxy approaches are described here: four different plankton-based proxies to reconstruct temperatures near the sea surface (near sea surface temperature or NSST), two plankton-based proxies to reconstruct sea ice distributions, and multi-proxy 
approaches.

Near sea surface temperatures ${ }^{1}$. The most extensive compilations of LGM NSST proxies are those of the CLIMAP [CLIMAP Project Members, 1976], GLAMAP [Sarnthein et al., 2003], and MARGO [Kucera et al., 2005a] projects. Two factors accounts for this focused attention on reconstructing LGM NSSTs: (1) as compared to other environmental variables, NSST is relatively straightforward to reconstruct from proxy evidence, and (2) as a key boundary condition for the atmosphere, NSST is an important parameter for realistic model simulations of LGM atmospheric conditions. The MARGO project, the most recent NSST compilation effort, developed comprehensive, global compilations based on a variety of proxy types. The project focused on making data accessible to modelers, and substantial effort was dedicated to providing quantitative uncertainties on each proxy record [Waelbroeck et al., 2009].

Due to their high quality and comprehensive nature, in the context of NSST reconstruction, only the MARGO compilations are considered here. The four MARGO compilations that provide some coverage in the Atlantic Ocean are described in this section: foraminiferal assemblages, dinocyst assemblages, $\mathrm{Mg} / \mathrm{Ca}$, and alkenones. The following applies to all four compilations. A relatively narrow definition of the LGM, from 23-19 cal ka, is used; this corresponds to the period of maximum continental ice extent [Kucera et al., 2005a]. To simplify comparisons, all modern calibrations used in MARGO were made using the 1998 World Ocean Atlas. Uncertainty estimates were assigned to all data; these incorporate uncertainties associated with dating, calibration, and with the number of samples available in the 23-19 kyr bp time slice. The specific details of MARGO uncertainty estimates are provided in Appendix A. Note that the evaluation of NSST proxies for model-data synthesis is postponed until all of the NSST proxies have been described.

NSSTs from assemblages of planktonic foraminifera. Planktonic foraminifera, or forams, are zooplankton that are found throughout the modern and Last Glacial Maximum

\footnotetext{
${ }^{1}$ NSST is used throughout this thesis in place of SST to avoid confusion with the usage of SST in meteorology as a term for the ocean's surface skin temperature. Note that in the paleoceanographic literature the term SST, and not NSST, is used.
} 
oceans. Although foraminifera make up a small fraction of all zooplankton, their $\mathrm{CaCO}_{3}$ shells constitute a major component of ocean sediments [Schiebel and Hemleben, 2005]. Assemblage or transfer-function based approaches are used to reconstruct near sea surface temperatures (or other climate parameters) based on the calcium carbonate $\left(\mathrm{CaCO}_{3}\right)$ shells of foraminifera recovered from sediment cores. The assumption used in assemblage and transfer function based approaches is that the number and type of planktonic species preserved in a sediment core can be used to infer climate conditions in the past. These techniques proceed as follows: (1) catalog the species assemblages of a variety of modern habitats for which instrumental records are available, (2) analyze the species assemblages of sediment samples from past climates, (3) identify similarities between the past climate assemblages and observed modern assemblages using multiple regression, and (4) use the regression to infer past climate conditions. Reconstruction of LGM NSSTs with foraminiferal assemblages has a long history (e.g. CLIMAP Project Members [1976], Pflaumann et al. [2003], Kucera et al. [2005b]). The MARGO compilation [Kucera et al., 2005b, Hayes et al., 2005] uses five geographically constrained calibration data sets, including three in the Atlantic: the South Atlantic ( $\mathrm{N}=321$ modern observations), North Atlantic $(\mathrm{N}=862)$, and Mediterranean Sea $(\mathrm{N}=145)$. This approach reduces noise and false associations between distinct populations.

Figure 2-3 (top row) shows maps of the difference between WOA'09 and MARGO foram assemblage based annual, winter, and summer mean LGM near sea surface temperatures; the same data are plotted against latitude in Figure 2-4 (top row). There are 174 foram assemblage based NSST records in the Atlantic domain considered in this thesis (in this thesis, "record" is used to refer to individual proxy data points, not to a time-series of data). Figure 2-3 (top row) reveals notable gaps in data coverage in the Gulf Stream, mid-latitude gyre, and Labrador Seas, and the data locations are biased towards continental margins. The WOA09 - MARGO anomalies show high spatial coherence, implying high spatial coherence in the MARGO foram assemblage based NSST reconstructions. Strong LGM cooling is evident in the subpolar gyre, especially south of Iceland; in the eastern half 
of the low-latitude oceans; and in the Norwegian Sea (northeast of Iceland) (Figure 2-3). Inferred temperature changes are small in the western half of the basin and in the Greenland Sea (northwest of Iceland). Annual, winter, and summer anomaly patterns are similar with one exception: the western equatorial region shows a stronger LGM cooling in the summer than in the winter. As is especially apparent in Figure 2-4, LGM cooling inferred from the foram assemblage based estimates is particularly large between $10^{\circ} \mathrm{S}-10^{\circ} \mathrm{N}$ and $40^{\circ} \mathrm{N}-70^{\circ} \mathrm{N}$ (see Figure 2-4).

Kucera et al. [2005b] applied three independent transfer function techniques to build independent reconstructions of LGM NSSTs: Artificial Neural Networks, Revised Analog Method, and SIMMAX (see Kucera et al. [2005b] for explanations); comparison of these independent estimates permits uncertainty estimation. Kucera et al. [2005b] note that while the three techniques agree well on some features (e.g. the strong subpolar gyre cooling), in other regions they disagree with each other; in regions with strong disagreement, the reconstructed values must be used with caution. In particular, north of $60^{\circ} \mathrm{N}$ (the Nordic Seas) the reconstructed summer temperatures show significant disagreement. Reconstructed temperatures for all seasons also vary widely between techniques from $15-25^{\circ} \mathrm{S}$ due to difficulty in reconstructing the Benguela Current and the southern subtropical gyre [Kucera et al., 2005b].

There are additional potential issues associated with foraminiferal assemblage based NSST reconstructions. As with all planktonic proxies, assemblages are likely biased towards periods of high productivity, whether seasonal (e.g. high-latitude spring blooms), or longer-term (e.g. especially mild decades or centuries during the LGM); these potential biases may be latitude (or more generally climate) dependent. Additionally, foraminifera are found at a variety of depths in the upper ocean, and some inhabit different depths at different phases of their life-cycle [Schiebel and Hemleben, 2005, Farmer et al., 2007]. A positive side to this issue is that it appears possible to use a combination of different species of foraminifera to reconstruct some aspects of upper ocean stratification [Farmer et al., 2007]. An issue specific to assemblage-based techniques is a no-analog situation: it 
Annual mean
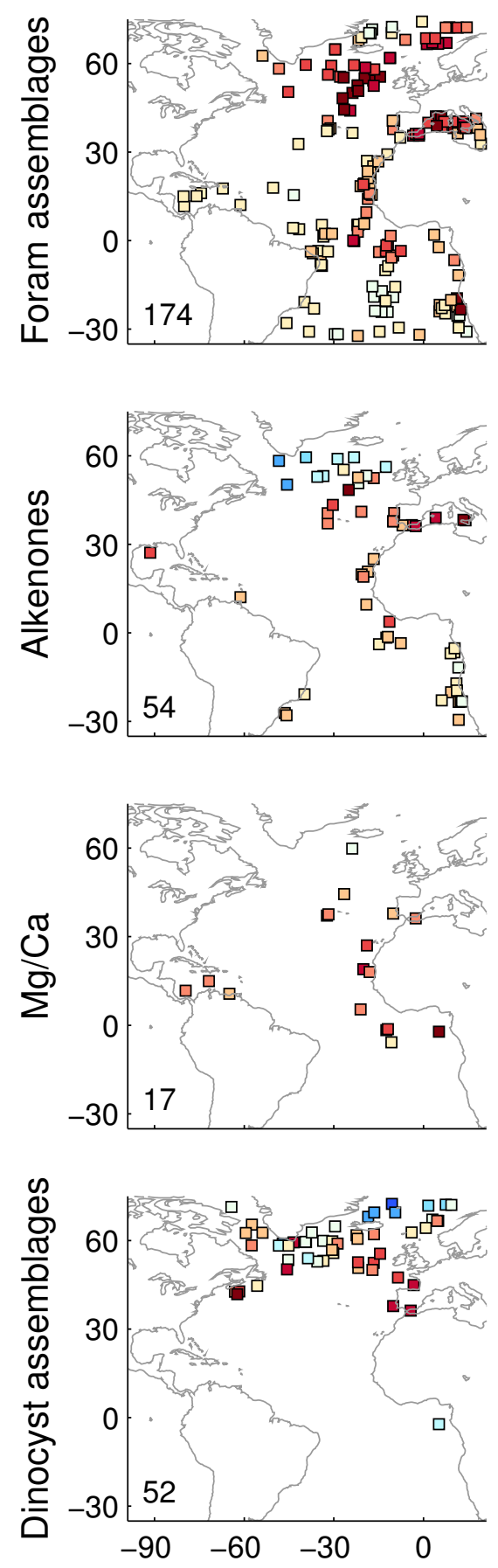

Winter mean
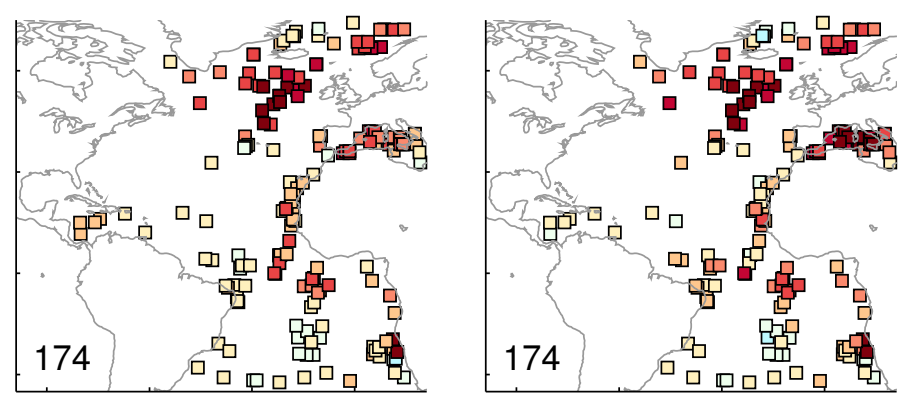
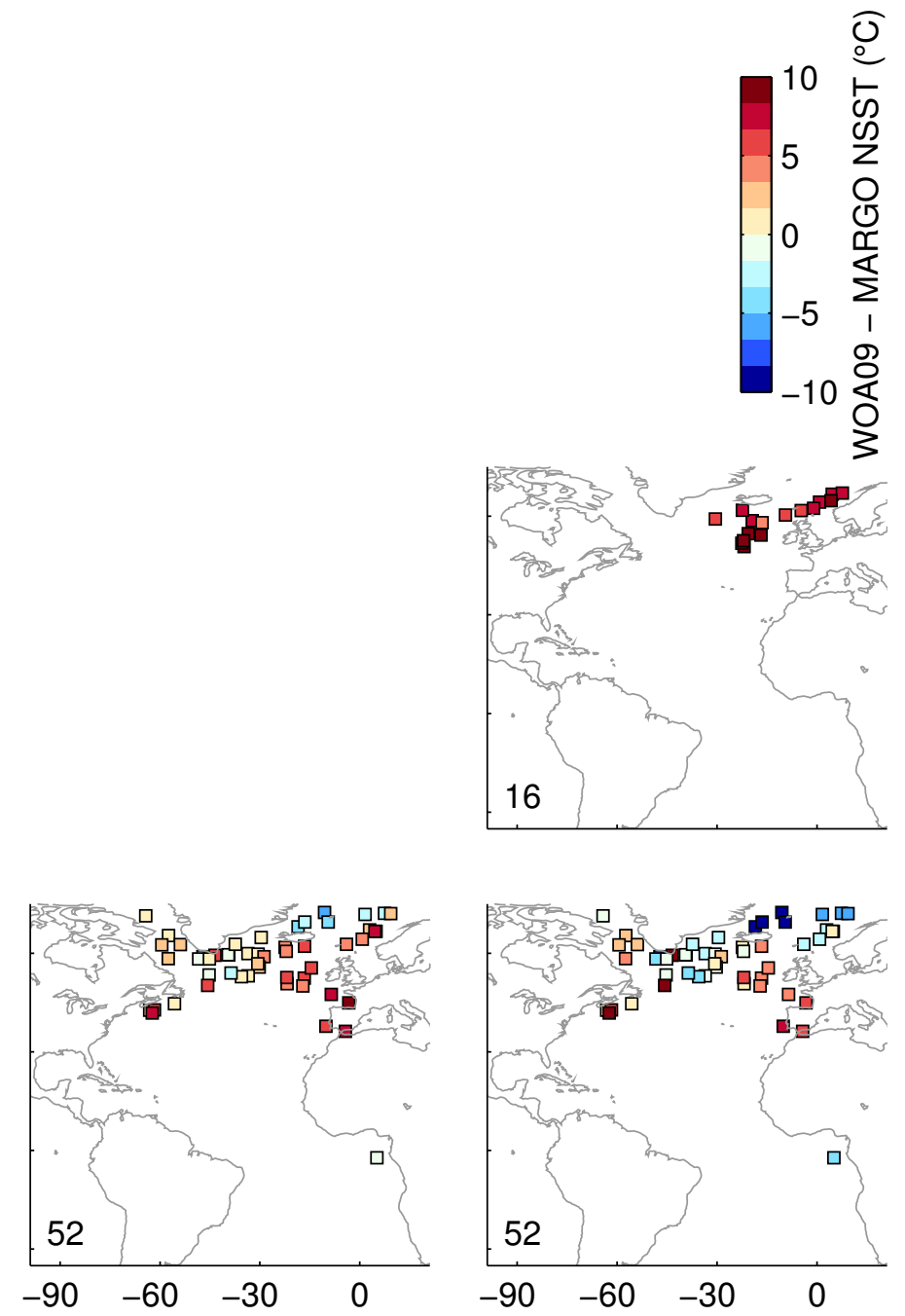

Figure 2-3: Difference in near sea surface temperatures between WOA'09 and MARGO data. The number of records in each plot is shown in the lower left-hand corners. Compilations are arranged top to bottom and seasonalities are arranged left to right. MARGO does not report winter/summer means for alkenones nor winter means for $\mathrm{Mg} / \mathrm{Ca}$. 

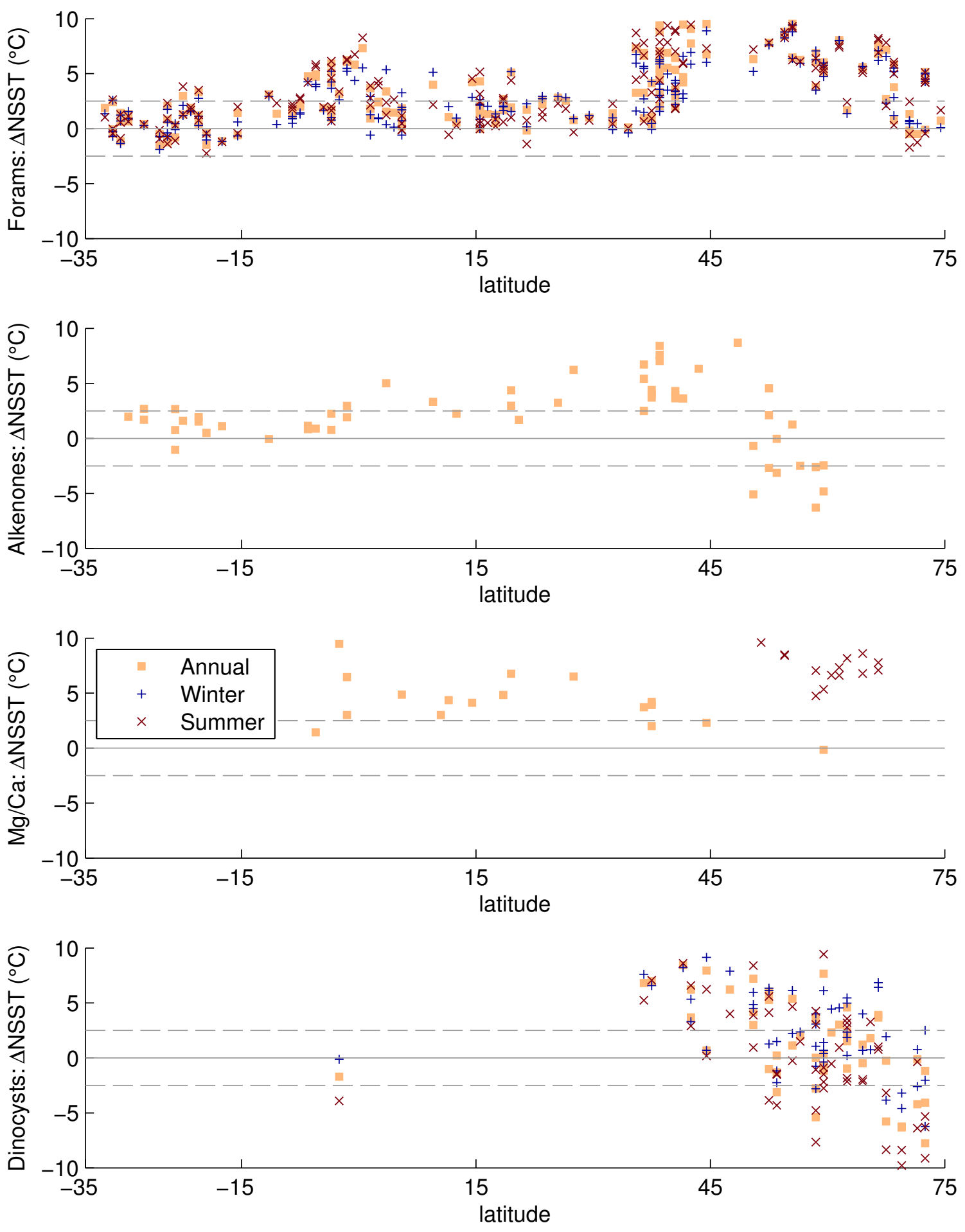

Figure 2-4: Difference in Atlantic Ocean near sea surface temperatures between WOA'09 and MARGO data plotted against latitude. Plots include anomalies for annual, winter, and summer (red crosses) mean NSSTs. Dashed lines at $\pm 2.5^{\circ} \mathrm{C}$ are included for reference. 
may be impossible to obtain a credible reconstruction of climate if an LGM assemblage does not resemble any of the modern assemblages in the database. This could be particularly problematic in the high-latitude N. Atlantic, which may have been marked by a fresh surface layer for much of the year at the LGM [De Vernal et al., 2006]; reconstruction may be particularly complicated because many foraminifera species do not thrive in low-salinity environments [De Vernal et al., 2006].

NSSTs from dinoflagellate cyst assemblages. Dinoflagellates are prevalent marine organisms found in environments from equatorial to polar and estuarine to oceanic [De Vernal et al., 2000]. Some dinoflagellate taxa are primary producers, while others are microzooplankton; all apparently live in a shallow surface layer [De Vernal et al., 2005]. Dinoflagellates themselves are not fossilizable, but dinoflagellate cysts are highly resistant to decay. These organic-walled cysts (dinocysts) are produced by some taxa to support a dormant period. Since only a subset of taxa produce cysts, transfer functions based on dinocysts represent a subset of the dinoflagellate population.

The modern reference database used for calibration in MARGO consists of 940 modern records covering the Mediterranean, northern N. Atlantic, Arctic, and N. Pacific. Canonical correspondence analysis (see De Vernal et al. 2005 for an explanation) was applied to the modern database to identify the dominant axes of variability in the modern records; cross-correlation between these axes and environmental conditions revealed that NSST and sea ice extent are primary controls on assemblage distributions, with salinity also playing a significant role. The accuracy of the dinocyst reconstruction approach is lower in ice marginal zones, estuaries, and on the continental margins, while better accuracy is achieved in open ocean regions where salinity is higher than 33 [De Vernal et al., 2005]. De Vernal et al. [2005] also note that, despite having an extensive modern calibration dataset with many Arctic and sub-Arctic samples, there is a clear lack of good analogues for Northern North Atlantic near sea surface conditions at the LGM. These authors hypothesize that the surface ocean in the high latitude Atlantic was much fresher at the LGM than it is today due to differences in the seasonal cycle of sea ice. 
The MARGO compilation of dinocyst-based NSSTs [De Vernal et al., 2005] includes 52 LGM records in our domain (see bottom row of Figure 2-3); Figure 2-4 (lower plot) shows the same data plotted against latitude. The dinocyst data in our domain are nearly all located north of $40^{\circ} \mathrm{N}$, and the WOA'09 NSST - dinocyst NSST anomalies indicate less spatial coherence than was the case for the foraminifera NSST estimates. Figure 2-4 shows strong LGM cooling between $35-50^{\circ} \mathrm{N}$, little change between $50-65^{\circ} \mathrm{N}$, and strong LGM warming between $65-75^{\circ} \mathrm{N}$; these data imply a large difference in high-latitude NSST gradients between the LGM and today. A change in seasonality is also inferred, with particularly large modern to LGM cooling in winter (Figure 2-4).

NSSTs from $\mathrm{Mg} / \mathrm{Ca}$ ratios of planktonic foraminifera. $\mathrm{Mg}$ can substitute for $\mathrm{Ca}$ in the $\mathrm{CaCO}_{3}$ during formation of a foraminifera shell; the ratio of $\mathrm{Mg}$ to $\mathrm{Ca}$ tends to increase exponentially with calcification temperature. Barker et al. [2005] compiled a variety of modern calibration equations, all of which follow the form $\mathrm{Mg} / \mathrm{Ca}=B e^{A \cdot T}$, where $\mathrm{T}$ is the calcification temperature in ${ }^{\circ} \mathrm{C}$ and $\mathrm{A}$ and $\mathrm{B}$ are species-dependent constants. The MARGO Mg/Ca compilation [Barker et al., 2005] includes 33 records in our domain, 17 of which are lower latitude and reported as annual mean and 16 of which are higher latitude and reported as summer mean (because high-latitude productivity is summer-biased). Measurements were made on mixed layer species G. ruber (w), G. sacculifer, G. bulloides, and N. pachyderma (s) and species-specific calibrations were used [Barker et al., 2005]. The MARGO Mg/Ca data are plotted in the third row of Figures 2-3 and 2-4. The data show very strong high-latitude summertime cooling at the LGM and moderate LGM cooling at low-latitudes.

A few details are important to interpreting these reconstructions. Since shallow-dwelling foraminifera complete their life cycles in 14 days (e.g. Globigerina bulloides) to 1 month (e.g. Globigerinoides ruber), each preserved test (shell) represents some averaging of conditions over a 2-4 week period. Typically, many tests are included in a $\mathrm{Mg} / \mathrm{Ca}$ analysis, so that the result is assumed to be an average over longer periods. $\mathrm{Mg} / \mathrm{Ca}$ ratios decrease with dissolution, perhaps because of preferential dissolution of high $\mathrm{Mg} / \mathrm{Ca}$ portions of 
the test [Barker et al., 2005]. Evidence of dissolution is apparent below the lysocline and little calcite is preserved below the calcite compensation depth (CCD); deeper cores are therefore likely to exhibit a dissolution-related cold-bias. Finally, since there is no standard procedure for laboratory preparation and analysis of samples, discrepancies between samples processed in different labs can lead to differences in reconstructed values of order $1^{\circ} \mathrm{C}$ [Barker et al., 2005].

NSSTs from Alkenones. The alkenone $\mathrm{U}_{37}^{K^{\prime}}$ index is a measure of the abundance of long versus short chain alkenones [Rosell-Melé et al., 2004], molecules that are produced by coccolithophores. Although the mechanism responsible for the variation of alkenone chain length with temperature is a matter of debate, laboratory and surface-sediment based empirical calibrations show a strong relationship between the index and temperature (see Rosell-Melé et al. [2004] for a discussion). As the index has been shown to correlate most highly with annual mean near sea surface temperature [Rosell-Melé et al., 2004], reconstructions are typically reported as reflecting this climate property. The MARGO alkenone-based NSST compilation [Rosell-Melé et al., 2004] includes 54 records in our domain; these data are plotted in the second row of Figures 2-3 and 2-4. The data show LGM temperature close to modern south of the equator, moderate LGM cooling from the equator to $45^{\circ} \mathrm{N}$, and LGM warming from $45-60{ }^{\circ} \mathrm{N}$.

There are complexities in interpreting the alkenone NSST proxy. The most basic is the lack of a mechanistic understanding of temperature's influence on alkenones, which makes it difficult to reason about the proxy's likely response in past climates. Also, while modern correlation studies link the index to annual mean near sea surface temperature, it is more likely that alkenones primarily record the most productive season: the warm season in high latitudes and the cold season in low latitudes [Schneider et al., 2010]. A number of concerns with application of alkenones in the Nordic Seas north of $65^{\circ} \mathrm{N}$ are raised by Rosell-Melé and Comes [1999]. Based on these concerns, the MARGO compilation excludes all alkenone-based reconstructions located north of $65^{\circ} \mathrm{N}$.

Evaluation of all NSST proxies for model-data syntheses. Each of the MARGO compi- 
lations of LGM NSST proxies satisfies the following requirements of a model-data synthesis: the data are publicly available, uncertainty estimates can be assigned to each record, and there is a clear mapping from properties that can be represented in a model (NSSTs) to properties reconstructed with the proxy. There are of course uncertainties and potential biases associated with the attribution of these proxies to NSST, and uncertainty estimates are imperfect; these issues must be considered, but do not prevent the use of the data in a model-data synthesis. The proxy types also vary in terms of the numbers of records and their geographic distribution (see Figure 2-3). If used together in a synthesis, uneven geographic distribution of proxy types that have unique biases could lead to a model-data synthesis with an unrealistic reconstruction of LGM ocean state. Relative weighting of the different proxy types is also an important consideration; results will depend on whether each individual proxy record is taken as independent and treated equally (in which case the foram assemblage based data will dominate), or whether equal weight is given to each of the four proxy types (in which case foram assemblages as a group are weighted at 25\%). With appropriate attention to these issues and considerations, the MARGO NSST compilations are well-suited to inclusion in model-data syntheses.

Sea ice distributions. It has been proposed that sea ice extent can be reconstructed from foram and dinocyst-based assemblage information, and these types of sea ice distribution estimates were made by the MARGO project [De Vernal et al., 2006]. Kucera et al. [2005b] argue that certain subpolar foraminifera species strictly require ice-free conditions for at least some of the year; their presence in an assemblage is then evidence of seasonally icefree conditions. Total absence of these foram species is believed indicative of perennial ice cover. As shown in Figure 2-5 (left), the perennial sea ice extent inferred from foram assemblages is modest, but the seasonal ice zone is relatively large, covering from the Greenland-Iceland-Norwegian (GIN) seas to Iceland and extending to the southern tip of Greenland.

Dinocyst-based sea ice distributions are shown on the right side of Figure 2-5. Perennial ice cover is indicated northwest of Iceland and in a broad swath along the coast of North 

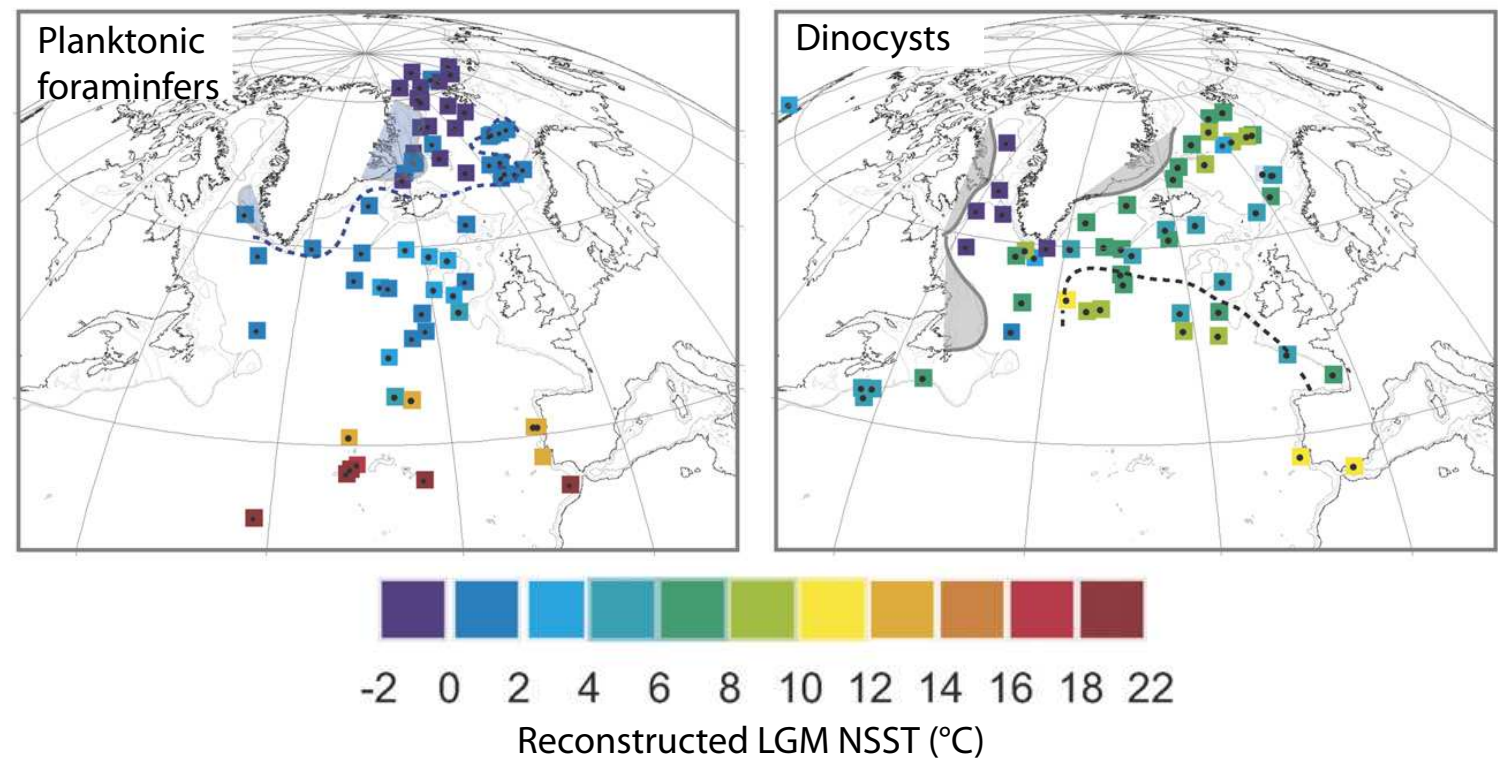

Figure 2-5: An adaptation of Figure 2 from De Vernal et al. [2006]. Reconstructed annual mean NSSTs at the LGM based on planktonic foraminifera and dinocysts. In the left diagram, blue shading marks regions with possible perennial LGM sea ice and the dashed line marks regions of seasonally ice-free conditions at the LGM. In the right diagram, grey shading marks perennial LGM sea ice and the dashed line marks the separation between seasonal sea ice and year-round ice-free conditions at the LGM.

America. The seasonal sea ice region extends deep into the subpolar gyre and far southward along the coast of Europe to northern France.

Evaluation for model-data syntheses. Sea ice is included in many models and modeldata synthesis with sea ice data would be of great value. Sea ice distributions are a strong indicator for a variety of related environmental parameters: sea ice regions are marked by NSSTs near the freezing point, air temperatures that are often extremely cold, and nearly absent air-sea fluxes of heat and other properties. The MARGO sea ice reconstruction is publicly available, and a reasonable geographic distribution of both dinocyst and foram assemblage based data is available. However, modern calibrations of sea ice proxies are less developed/robust than are modern NSST calibrations, and uncertainty estimates are not available. Also, while sea ice is included in many models, care must be taken in mapping model variables such as sea ice fraction or sea ice thickness to estimates that can be directly 
compared to the more qualitative proxy estimates (e.g. seasonal versus perennial). Inclusion of these data in model-data syntheses would be more difficult than for NSST proxies. Given the potential to constrain important properties of the climate system, the effort would be worthwhile.

Inferences based on multiple proxies. Some authors have employed multiple types of proxies to reconstruct upper ocean conditions at the LGM. Such multi-proxy approaches have the potential to reconstruct more climate characteristics than is possible with a single proxy type, but can also be more difficult to interpret as the uncertainties of each proxy type must be considered in interpreting the results. It is beyond the scope of this chapter to describe in detail such multi-proxy approaches; instead several examples of such studies are mentioned as a starting point for the interested reader.

De Vernal et al. [2002] combined dinocyst assemblage information with $\delta^{18} \mathrm{O}_{\text {calcite }}$ measurements to estimate near sea surface temperature, salinity, potential density, and sea ice presence. Meland et al. [2005] combined planktonic $\delta^{18} \mathrm{O}_{\text {calcite }}$ records and $\mathrm{Mg} / \mathrm{Ca}$-based estimates of NSST to obtain estimates of near sea surface salinity (NSSS) in the Northern North Atlantic and Nordic Seas; the $\mathrm{Mg} / \mathrm{Ca}$ and $\delta^{18} \mathrm{O}_{\text {calcite }}$ measurements were made on the same planktonic foraminifera shells, reducing or removing many sources of uncertainty that would be present in using proxy records measured on different source materials. Waelbroeck et al. [2009] employed all of the MARGO NSST compilations to create a gridded LGM NSST estimate at $5^{\circ} \times 5^{\circ}$ horizontal resolution; uncertainties were also estimated for each grid box.

Evaluation for model-data syntheses. In creating model-data syntheses, the data should be as close to a direct measurement as possible - the model itself serves as the interpretation tool. The advantages of multi-proxy inferences can be obtained in a model-data synthesis by incorporating each proxy on its own. For example, a combination of $\mathrm{Mg} / \mathrm{Ca}$-based NSST estimates with planktonic $\delta^{18} \mathrm{O}_{\text {calcite }}$ measurements is best done by constraining modeled NSSTs to the $\mathrm{Mg} / \mathrm{Ca}$ records and building an isotope model to use in conjunction with the $\delta^{18} \mathrm{O}_{\text {calcite }}$ records. 


\subsubsection{Deep ocean state}

Deep ocean proxies are defined here to be those that record conditions near the sedimentwater interface; the distinction is largely practical - in addition to recording information about the upper ocean as reviewed in the last section, sediment cores also preserve bottom dwelling organisms and properties of the sediment itself. Proxies for glacial deep ocean circulation have been reviewed by Lynch-Stieglitz [2003] and were the focus of a 2008 workshop focused on compiling deep circulation proxies for the LGM (see Paul and Mulitza 2009; note that this compilation effort is not yet complete).

This section summarizes the most promising deep ocean proxies for model-data synthesis. The reviewed proxies include properties that are associated with the sediment itself (grain size based flow speed proxies, ratios of protactinium to thorium, $\epsilon_{\mathrm{Nd}}$ of thin ferromanganese coatings on sediment particles), properties of the fluid preserved with the sediment (pore fluid measurements), or properties measured in the remains of benthic organisms $\left(\delta^{18} \mathrm{O}, \delta^{13} \mathrm{C}, \Delta{ }^{14} \mathrm{C}, \epsilon_{\mathrm{Nd}}\right.$, and $\mathrm{CaCO}_{3}$ substitution ratios of $\mathrm{Mg} / \mathrm{Ca}, \mathrm{Zn} / \mathrm{Ca}$, and $\left.\mathrm{Cd} / \mathrm{Ca}\right)$. As was done for upper ocean proxies, an evaluation is given for each proxy type of whether the proxy is appropriate at this point for model-data syntheses and what special efforts, if any, are required to incorporate each proxy type into such a synthesis.

Oxygen isotopic ratios. The use of oxygen isotopes as a proxy for past oceanic conditions has been a mainstay of the paleoceanographic community for several decades. $\delta^{18} \mathrm{O}$ is a measurement of the relative ratio of the less common ${ }^{18} \mathrm{O}$ to the more common ${ }^{16} \mathrm{O}$ and is reported relative to the PDB standard (Cretaceous belemnite from the Pee Dee River formation in North Carolina, USA) for carbonate samples and relative to the SMOW standard (Standard Mean Ocean Water) for water samples [Waelbroeck et al., 2005].

$$
\delta^{18} \mathrm{O}(\% 0)=\frac{\left({ }^{18} \mathrm{O} /{ }^{16} \mathrm{O}\right)_{\text {sample }}-\left({ }^{18} \mathrm{O} /{ }^{16} \mathrm{O}\right)_{\text {standard }}}{\left({ }^{18} \mathrm{O} /{ }^{16} \mathrm{O}\right)_{\text {standard }}} \times 10^{3}
$$

The $\delta^{18} \mathrm{O}_{\text {water }}$ of the upper ocean is strongly influenced by evaporation, precipitation, and runoff (including the melting of continental ice). Evaporation favors lighter isotopes, 
leaving the surface ocean isotopically heavier, while precipitation and runoff are isotopically light relative to mean seawater. Atmospheric water vapor becomes isotopically lighter when precipitation preferentially removes heavy isotopes (Rayleigh distillation), a process that leads to reduction in the $\delta^{18} \mathrm{O}$ of rainwater with distance from the evaporative source. Rayleigh distillation leads to an inverse correlation of $\delta^{18} \mathrm{O}_{\text {precipitation }}$ with latitude, altitude, and distance from the evaporative source. In summary, regions where evaporation dominates precipitation (E-P $>0$ ) tend to have higher $\delta^{18} \mathrm{O}_{\text {water }}$ than regions where E-P $<0$, and low latitude waters tend to have higher $\delta^{18} \mathrm{O}_{\text {water }}$ than high latitude waters. The net effect of evaporation and precipitation has a similar impact on salinity and $\delta^{18} \mathrm{O}_{\text {water }}$, which often leads to a positive correlation of ocean salinity and $\delta^{18} \mathrm{O}_{\text {water }}$. In the modern ocean, water column $\delta^{18} \mathrm{O}$ can be measured directly and a compilation of over 24,000 measurements is available [Schmidt et al., 1999].

The global mean $\delta^{18} \mathrm{O}$ ratio of seawater, $\delta^{18} \mathrm{O}_{\text {water }}$, is expected to vary over the glacialinterglacial cycles because land ice is isotopically light, leaving the ocean isotopically heavier during periods with more extensive terrestrial ice sheets. Duplessy et al. [2002] use sediment pore fluid records (see page 61 for a discussion of this proxy) and highlatitude benthic foraminiferal $\delta^{18} \mathrm{O}_{\text {calcite }}$ records to estimate that mean ocean deep waters were $1.05 \pm 0.20 \%$ heavier at the LGM than today.

Other than the few estimates of LGM $\delta^{18} \mathrm{O}_{\text {water }}$ that have been made from pore fluid measurements in sediment cores, the bulk of available records are measurements of $\delta^{18} \mathrm{O}$ in the $\mathrm{CaCO}_{3}$ of planktonic and benthic foraminifera $\left(\delta^{18} \mathrm{O}_{\text {calcite }}\right)$. Due to a temperature dependent fractionation effect that occurs during formation of biogenic $\mathrm{CaCO}_{3}$, the $\delta^{18} \mathrm{O}_{\text {calcite }}$ of foraminifera depends on both the ambient $\delta^{18} \mathrm{O}_{\text {water }}$ and on the ambient water temperature. Excluding other factors, foraminifera formed in colder waters have a higher $\delta^{18} \mathrm{O}_{\text {calcite }}$. Modern calibrations have been used to develop a variety of paleo temperature equations (see Bemis et al. [1998] for a review). As an example, Shackleton [1974] reported

$$
T=16.9-4.38 \cdot\left(\delta^{18} \mathrm{O}_{\text {calcite }}-\delta^{18} \mathrm{O}_{\text {water }}\right)+0.1 \cdot\left(\delta^{18} \mathrm{O}_{\text {calcite }}-\delta^{18} \mathrm{O}_{\text {water }}\right)^{2}
$$


where $T$ is the inferred temperature of calcification in ${ }^{\circ} \mathrm{C}$.

The most complete published compilation of LGM $\delta^{18} \mathrm{O}_{\text {calcite }}$ measurements in benthic foraminifera is that of Marchal and Curry [2008]; this compilation is Atlantic-focused and includes 180 records of LGM age (defined as 18-21 kyr BP by these authors) in addition to 198 records of Holocene age (0-3 kyr BP). As shown in Figure 2-6, about half of the cores in the compilation originate in water depths greater than $2500 \mathrm{~m}$. Section 2.2.1 includes a detailed analysis of uncertainties in $\delta^{18} \mathrm{O}_{\text {calcite }}$ as a recorder of water column properties. Marchal and Curry [2008] assume an overall uncertainty of $0.2 \%$; this estimated uncertainty is significantly larger than expected instrumental precision to compensate for the potential interlaboratory calibration differences identified by Ostermann and Curry [2000]. See appendix in Marchal and Curry [2008] for further discussion of this uncertainty estimate.

Lynch-Stieglitz et al. [1999a] reconstruct density from Holocene $\delta^{18} \mathrm{O}_{\text {calcite }}$ measurements to estimate the modern geostrophic transport through the Florida Straits. This calibration is then used in Lynch-Stieglitz et al. [1999b] to estimate that LGM transport through the Florida Straits was only about $2 / 3$ of the modern transport. To reconstruct density, an empirical relationship is identified between $\delta^{18} \mathrm{O}_{\text {calcite }}$ and potential density as follows [Lynch-Stieglitz et al., 1999a]. Salinity data from GEOSECS stations near the Florida Straits are used to predict $\delta^{18} \mathrm{O}_{\text {water }}$ based on an empirically determined relationship between $\delta^{18} \mathrm{O}_{\text {water }}$ and salinity. The empirical dependence of $\delta^{18} \mathrm{O}_{\text {calcite }}$ on $\delta^{18} \mathrm{O}_{\text {water }}$ and temperature is then used along with temperature data from nearby GEOSECS stations to estimate $\delta^{18} \mathrm{O}_{\text {calcite }}$. This computation provides an empirical relationship between $\delta^{18} \mathrm{O}_{\text {calcite }}$ and density. Next, this relationship is used to estimate density profiles at Florida Keys and Little Bahama Bank; these profiles are then used in an approximate geostrophic calculation with an assumed level of no motion at the bottom. This method provides an estimate of $32 \mathrm{~Sv}$ for the modern mean annual volume transport, an estimate the authors note is in agreement with estimates from other approaches that yield 30-32 Sv. Lynch-Stieglitz et al. [1999a] note that this approach is most applicable for warm waters $\left(\geq 5^{\circ} \mathrm{C}\right)$ that are rela- 

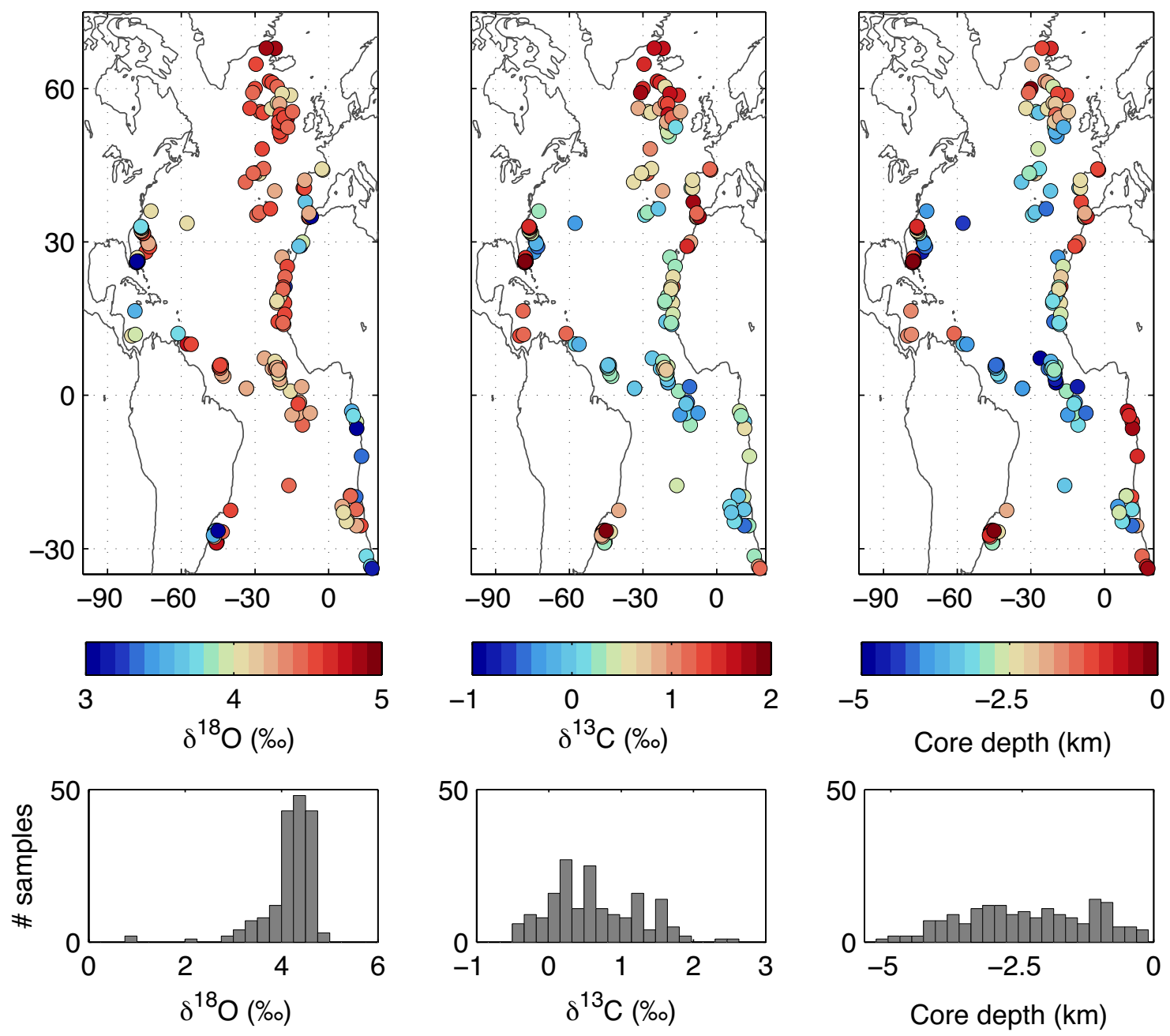

Figure 2-6: LGM $\delta^{18} \mathrm{O}_{\text {calcite }}$ (left), $\delta^{13} \mathrm{C}_{\text {calcite }}$ (middle), and core depths (right) from the Marchal and Curry [2008] compilation. Data are shown in map form in the top row (color limits may be cropped). Histograms of all values are shown in the bottom row (full data range is included).

tively shallow. The method is inappropriate at cold temperatures and for deeper waters for a variety of reasons: lines of equal density become far from parallel with lines of constant $\delta^{18} \mathrm{O}_{\text {calcite }}$ at low temperatures, density contrasts in the abyssal ocean are small and cannot be differentiated with $\delta^{18} \mathrm{O}_{\text {calcite }}$ given its uncertainties, and processes unique to polar regions such as sea ice formation lead to large errors when using standard $\delta^{18} \mathrm{O}_{\text {water }} /$ salinity relationships. The validity of this approach to reconstructing paleo transports has been 
questioned [Wunsch, 2003].

Evaluation for model-data syntheses. The Marchal and Curry [2008] compilation provides a readily accessible, quality-controlled dataset for model-data comparison with an uncertainty estimate based on calibration efforts (a uniform uncertainty of $0.2 \%$ is applied). An important restriction is that this compilation is Atlantic-only, and we are not aware of any global compilations. A priori mapping of $\delta^{18} \mathrm{O}_{\text {water }}$ to density, as in the work of Lynch-Stieglitz et al. [1999b], is not recommended for model-data syntheses; instead, the data should ideally be used without significant prior interpretation (so that interpretations made from the model-data synthesis are as direct as possible). One key complication of using the data without interpretation in a model-data syntheses is that $\delta^{18} \mathrm{O}_{\text {calcite }}$ is strongly dependent on both ambient temperature and $\delta^{18} \mathrm{O}_{\text {water }}$. State estimation with fully coupled simulations that integrate complete isotope models are the ideal tool for use of these data, but such tools are presently not available (and may not be for some time). In the meantime, two approaches are possible. In the first, it can be argued that temperature and $\delta^{18} \mathrm{O}_{\text {water }}$ are conservative tracers once a water mass leaves the surface, so that $\delta^{18} \mathrm{O}_{\text {calcite }}$ can be treated as nearly conservative; one must neglect that $\mathrm{CaCO}_{3}$ actually records in-situ temperature rather than potential temperature (see page 14 of Lund et al. 2011 for a discussion of the potential errors associated with this effect in the Atlantic). In this case, $\delta^{18} \mathrm{O}_{\text {calcite }}$ can be modeled as a conservative tracer. In the second approach, both temperature and $\delta^{18} \mathrm{O}_{\text {water }}$ are modeled independently (with $\delta^{18} \mathrm{O}_{\text {water }}$ modeled as a passive, conservative tracer) and $\delta^{18} \mathrm{O}_{\text {calcite }}$ is estimated from these two modeled quantities. In either case, these data can be incorporated in model-data syntheses with a moderate effort and the dataset is large enough to make such an effort worthwhile.

Carbon isotopic ratios. Like oxygen isotopes, carbon isotopic ratios have been an important proxy for several decades. $\delta^{13} \mathrm{C}$ is a measure of the relative ratio of ${ }^{13} \mathrm{C}$ to ${ }^{12} \mathrm{C}$ :

$$
\delta^{13} \mathrm{C}(\% 0)=\frac{\left({ }^{13} \mathrm{C} /{ }^{12} \mathrm{C}\right)_{\text {sample }}-\left({ }^{13} \mathrm{C} /{ }^{12} \mathrm{C}\right)_{\text {standard }}}{\left({ }^{13} \mathrm{C} /{ }^{12} \mathrm{C}\right)_{\text {standard }}} \times 10^{3}
$$

where the standard is Pee Dee Belemnite. In the ocean, about 99\% of the dissolved inor- 
ganic carbon (DIC) is in the form of ${ }^{12} \mathrm{C}$. Light carbon is preferentially incorporated into organic matter during photosynthesis. For this reason, organic matter in the ocean has a mean $\delta^{13} \mathrm{C}$ value around $-22 \%$, though this value varies from about $-19 \%$ in warmer regions to -30\% in colder regions [Lynch-Stieglitz, 2003]. When surface ocean DIC equilibrates with atmospheric $\mathrm{CO}_{2}$ through air-sea exchange, a fractionation effect leaves the ocean isotopically heavier; this effect is temperature dependent, causing higher surface ocean $\delta^{13} \mathrm{C}_{\text {DIC }}$ cold regions. Since the timescale of this equilibration process (about 10 years, Lynch-Stieglitz [2003]) is longer than typical surface ocean timescales, equilibrium is never reached.

Like fractionation during air-sea $\mathrm{CO}_{2}$ exchange, photosynthesis preferentially removes light carbon, leaving the surface ocean isotopically heavier. The combined effect of airsea fluxes and surface ocean photosynthesis produces relatively high $\delta^{13} \mathrm{C}_{\mathrm{DIC}}$ levels in the upper ocean. In deeper layers, remineralization of organic matter introduces light organic matter, thus lowering $\delta^{13} \mathrm{C}_{\text {DIC }}$. Because these processes affect $\delta^{13} \mathrm{C}_{\mathrm{DIC}}$ in a similar way as they do a nutrient like phosphate, the modern $\delta^{13} \mathrm{C}_{\text {DIC }}$ distribution is nutrient-like [LynchStieglitz, 2003]. Due to the relatively short residence time of waters in the deep Atlantic (see page 135 for a discussion), the initial $\delta^{13} \mathrm{C}_{\mathrm{DIC}}$ signatures of the water mass formation regions can be a much stronger signal in a deep water sample than the effect of remineralization. For study of the modern and LGM deep Atlantic Ocean, $\delta^{13} \mathrm{C}_{\mathrm{DIC}}$ is therefore sometimes treated as a nearly conservative tracer of water mass mixing [Lynch-Stieglitz, 2003].

As was the case for $\delta^{18} \mathrm{O}_{\text {calcite }}$, the Marchal and Curry [2008] compilation of $\delta^{13} \mathrm{C}_{\text {calcite }}$ is a large, quality controlled $\delta^{13} \mathrm{C}_{\text {calcite }}$ dataset with a tight time restriction on the LGM period (18-21 kyr BP). The middle plot of Figure 2-6 shows the LGM $\delta^{13} \mathrm{C}_{\text {calcite }}$ records from this compilation. A more recent compilation by Hesse et al. [2011] adds 40 additional records to the Marchal and Curry [2008] for a total of 220 LGM $\delta^{13} \mathrm{C}_{\text {calcite }}$ records; the age range used by Hesse et al. [2011] is 18-24 kyr BP, longer than that used by Marchal and Curry [2008]. Oliver et al. [2010] produced a benthic $\delta^{13} \mathrm{C}_{\text {calcite }}$ compilation focused on reconstructing the 
past 150,000 years and includes all regions of the ocean. To achieve these broader spatial and temporal goals, this compilation has weaker dating requirements and coarser temporal resolution than those of Hesse et al. [2011] and Marchal and Curry [2008].

Many of the sources of uncertainty that were reviewed for $\delta^{18} \mathrm{O}_{\text {calcite }}$ in Section 2.2.1

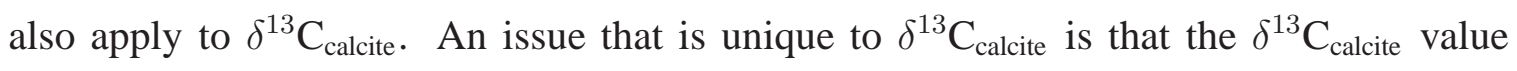
recorded by benthic species depends on the microhabitat of the species: species that live on or just above the sediment record the bottom water $\delta^{13} \mathrm{C}_{\text {DIC }}$ more faithfully, while species that live within the sediment tend to record values that are more negative due to the effect of remineralization in the sediment [Lynch-Stieglitz, 2003]. Many studies now rely on Cibicidoides or Planulina genera because they are thought to most faithfully record bottom water $\delta^{13} \mathrm{C}_{\text {calcite }}$ [Lynch-Stieglitz, 2003]. The laboratory reproducibility or external precision of $\delta^{13} \mathrm{C}_{\text {calcite }}$ measurements is about $0.04 \%$. To account for other uncertainties associated with the proxy, Marchal and Curry [2008] assign an uncertainty of 0.1 to $0.2 \%$ on their $\delta^{13} \mathrm{C}_{\text {calcite }}$ compilation. A more extensive discussion of $\delta^{13} \mathrm{C}_{\text {calcite }}$ uncertainties is provided by Oliver et al. [2010].

Evaluation for model-data syntheses. There are two compilations of Atlantic $\delta^{13} \mathrm{C}_{\text {calcite }}$ records for the LGM and reasonable uncertainty estimates exist. As there are not currently any global compilations, model-data synthesis efforts with $\delta^{13} \mathrm{C}_{\text {calcite }}$ are restricted to the Atlantic. Again, state estimation with a fully coupled model that includes an isotope model (and a carbon cycle model) would be ideal. Since this capability will not be available for some time, a simplified approach is necessary. A reasonable approximation can be obtained by treating $\delta^{13} \mathrm{C}_{\mathrm{DIC}}$ as a tracer impacted only by remineralization and ocean transport processes, as was done in an inverse modeling approach used by Marchal and Curry [2008].

Radiocarbon. Radiocarbon is a naturally occurring carbon isotope $\left({ }^{14} \mathrm{C}\right)$ that is generated cosmogenically in the atmosphere, and which decays in time with a half-life of 5730 years. Estimated oceanic $\Delta^{14} \mathrm{C}$ is obtained by correcting $\delta^{14} \mathrm{C}$ for isotopic fractionation effects using $\delta^{13} \mathrm{C}$. Oceanic $\Delta^{14} \mathrm{C}$ distributions are produced by spatially and temporally variable surface ocean $\Delta^{14} \mathrm{C}$ levels, aging of water masses once they are isolated 
from the surface ocean, and ocean advection and mixing. Surface ocean $\Delta^{14} \mathrm{C}$ levels are variable because the atmospheric generation rate of ${ }^{14} \mathrm{C}$ varies in time [Reimer and coauthors, 2004] and because atmosphere-ocean equilibration times are often longer than surface ocean residence times, so that the surface ocean only partially equilibrates with the atmosphere [Lynch-Stieglitz, 2003].

Although bottom water radiocarbon is recorded by benthic foraminifera and other organisms such as corals, ${ }^{14} \mathrm{C}$ continues to decay after these biogenic materials are buried on the sea floor. In order to use benthic $\Delta^{14} \mathrm{C}$ records to infer water mass ages, an independent estimate of the age of the core sample is required, complicating use of the proxy. Interpretation of radiocarbon records is also complicated by the fact that surface ocean $\Delta^{14} \mathrm{C}$ has changed significantly over the past 30,000 years, so that deep ocean $\Delta^{14} \mathrm{C}$ proxy records are a combination of variable surface boundary conditions and unknown water mass transport and mixing histories.

Although the number of oceanic $\Delta^{14} \mathrm{C}$ records has grown quickly over the past decade, as of yet there are no publicly available compilations of $\Delta^{14} \mathrm{C}$ records for the LGM. We are however aware of several groups that plan to undertake compilations of records that span from before the LGM to the late Holocene.

Evaluation for model-data syntheses. Radiocarbon records have the potential to constrain ocean transport timescales, making this proxy appealing for model-data synthesis. However, no publicly available compilations exist, and interpretation of the proxy is complicated. Inclusion of this proxy in a data-model synthesis would be worth consideration, but might require significant effort.

Pore-fluid measurements of $\delta^{18} \mathbf{O}_{\text {water }}$ and salinity. Ocean sediment cores contain seawater in the pores surrounding the sediment. McDuff [1985] was the first to recognize the signal of glacial cycles in pore fluids after measuring these pore fluids on Deep Sea Drilling Project site 576 in the central Pacific. Over time, measurements of $\delta^{18} \mathrm{O}$ and/or chlorinity (to reconstruct salinity) were made on pore fluids from sediment cores collected in a variety of locations [Schrag and DePaolo, 1993, Burns and Maslin, 1999, Schrag et al., 
1996, Adkins and Schrag, 2001, Schrag et al., 2002, Adkins et al., 2002, Adkins and Schrag, 2003]. The approach assumes that the evolution of pore fluid properties over time can be described using a vertical advection/diffusion equation with assumed boundary conditions at the sediment-water interface and deep in the sediment. Fixed values are chosen for all parameters except the diffusivity and amplitude of property variations at the sediment-water interface. These two properties are then found by calculating the best fit of the modeled and observed pore fluid properties with depth. An alternative, inverse approach has been used to reconstruct terrestrial temperatures from borehole temperatures [Mareschal and Beltrami, 1992, Beltrami and Mareschal, 1997], but to our knowledge has not been applied to ocean sediment data.

Figure 2-7 summarizes the six Atlantic sediment cores most relevant to our study. These records have been used to infer a global mean $\delta^{18} \mathrm{O}_{\text {water }}$ change between the LGM and today of $1.0 \pm 0.1 \%$ [Schrag et al., 1996]. Moreover, deep ocean temperatures were inferred to be at or near the freezing point of seawater [Adkins and Schrag, 2001], and AABW was inferred to be much saltier at the LGM than today [Adkins et al., 2002]. If applicable to the basin-scale, these inferences would imply that the ocean was more salt-stratified at the LGM than today.

Evaluation for model-data syntheses. The pore fluid records shown in Figure 2-7 can be easily incorporated in model-data syntheses efforts, limited only by whether the model in question simulates bottom water temperature, salinity, and/or $\delta^{18} \mathrm{O}$. The primary drawback is that few measurements of pore fluid of glacial age have been made. Apparently, a recent series of pore fluid measurements on Pacific sediment cores does support the existence of high AABW salinities at the LGM (Art Spivack, personal communication), but at this time those results remain unpublished.

Neodymium isotopic ratios. Neodymium is a trace metal in the ocean with two prevalent isotopes: (1) primordial ${ }^{144} \mathrm{Nd}$ and (2) ${ }^{143} \mathrm{Nd}$, which is formed by radioactive decay of ${ }^{147} \mathrm{Sm}$ (samarium, $\mathrm{t}_{1 / 2}=1.06 \times 10^{11}$ years). Neodymium isotopic ratios are typically 


\begin{tabular}{|l|l|l|l|}
\hline Site & ${ }^{18} \mathrm{O}_{\text {sw }}(\% \circ)$ & $\mathrm{S}$ & $\mathrm{T}\left({ }^{\circ} \mathrm{C}\right)$ \\
\hline $984^{2}$ & $0.8 \pm 0.1$ & & -1.5 \\
\hline $981^{3}$ & $\begin{array}{l}1.05 \pm 0.1^{3} \\
0.7 \pm 0.1^{2}\end{array}$ & $36.10 \pm 0.10$ & $-1.2 \pm 0.2$ \\
\hline $1063^{3}$ & $0.75 \pm 0.1$ & $35.83 \pm 0.03$ & $-2.2 \pm 0.5^{4}$ \\
\hline $925^{1}$ & $0.8 \pm 0.1$ & & \\
\hline $1088^{2}$ & $0.75 \pm>0.1$ & & \\
\hline $1093^{3}$ & $1.17 \pm 0.1$ & $37.09 \pm 0.17$ & $-1.3 \pm 0.9$ \\
\hline
\end{tabular}

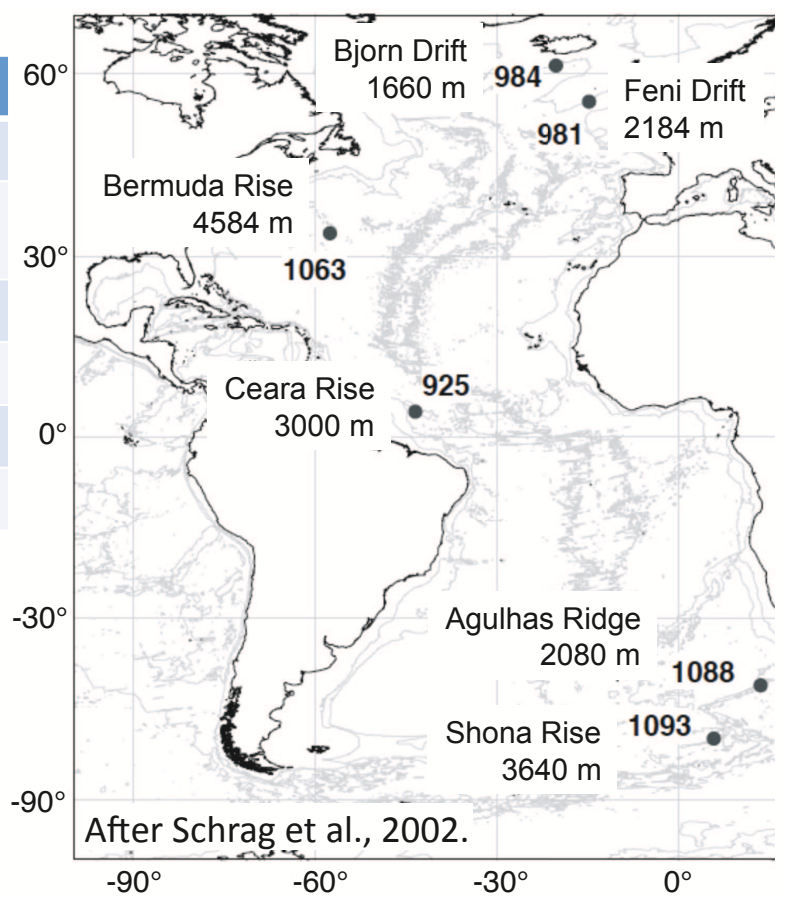

Figure 2-7: Summary of LGM pore-fluid measurements. Table (left) is a summary compiled from [Schrag et al., 1996, 2002, Adkins et al., 2002]. Some table cells are empty because temperature and salinity were not estimated for all sites. Figure (right) is a modified version from Schrag et al. [2002] (their Fig. 1).

expressed as

$$
\epsilon_{\mathrm{Nd}}=\left[\frac{\left({ }^{143} \mathrm{Nd} /{ }^{144} \mathrm{Nd}\right)_{\text {sample }}}{\left({ }^{143} \mathrm{Nd} /{ }^{144} \mathrm{Nd}\right)_{\text {standard }}}-1\right] \cdot 10^{4}
$$

The ratio of ${ }^{143} \mathrm{Nd}$ to ${ }^{144} \mathrm{Nd}$ in rocks reflects their relative composition of neodymium and samarium as well as their age [Frank, 2011]. Jeandel et al. [2007] compiled modern measurements of $\epsilon_{\mathrm{Nd}}$ and developed a map of $\epsilon_{\mathrm{Nd}}$ values for modern source regions to the oceans (i.e. along the coastlines).

The oceanic distribution of $\epsilon_{\mathrm{Nd}}$ is controlled by the composition of inputs from continental weathering, mantle sources, and aeolian inputs. Regional differences in $\epsilon_{\mathrm{Nd}}$ sources cause the modern ocean basins to have distinct $\epsilon_{\mathrm{Nd}}$ signatures (Lacan et al. [2012], see left side of Figure 2-8). To reconstruct past ocean properties, $\epsilon_{\mathrm{Nd}}$ is measured in marine precipitates as an estimate of the isotopic composition of the seawater at the time of 

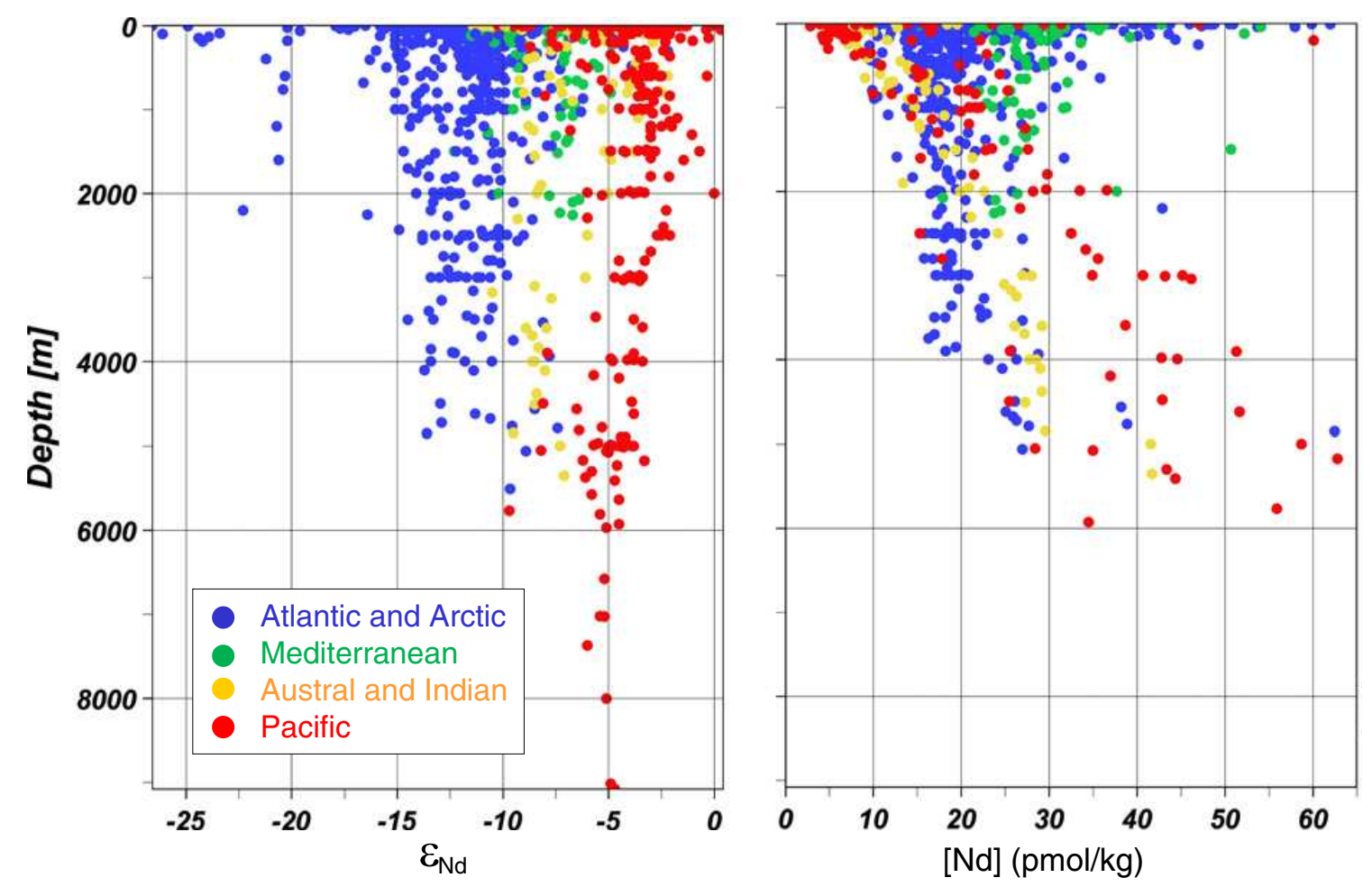

Figure 2-8: Modern $\epsilon_{\mathrm{Nd}}$ (left) and neodymium concentration (right) for four ocean basins. Figure from Lacan et al. [2012]

precipitation. Measurements have been carried out on sedimentary ferromanganese oxide leachates, benthic and planktonic foraminifera, fish teeth, deep sea corals, and ferromanganese crusts [Frank, 2011]. Note that the $\epsilon_{\mathrm{Nd}}$ ratio is thought to be unaffected by biological fractionation [Frank, 2011].

It is often assumed that away from sources and sinks of neodymium, $\epsilon_{\mathrm{Nd}}$ can be considered as a quasi-conservative tracer affected primarily by transport processes (e.g. Frank [2011]). However, $\epsilon_{\mathrm{Nd}}$ is a ratio of two concentrations, and while each concentration may individually satisfy an advection/diffusion equation, their ratio instead satisfies a highly nonlinear equation that requires additional boundary condition information. This issue could be neglected if the denominator concentration does not vary much in the ocean. As shown on the right side of Figure 2-8, the concentration of neodymium in modern seawater varies over an order of magnitude. 
Evaluation for model-data syntheses. Models of $\epsilon_{\mathrm{Nd}}$ have been incorporated in general circulation models [Jones et al., 2008, Arsouze et al., 2010] and a recent compilation of 880 modern $\epsilon_{\mathrm{Nd}}$ measurements [Lacan et al., 2012] should aid in efforts to disentangle processes affecting $\epsilon_{\mathrm{Nd}}$ in the modern ocean. However, this proxy is still relatively new and, as concluded in a review by Frank [2011], more development is needed to fully understand modern cycling of neodymium and how to use $\epsilon_{\mathrm{Nd}}$ to reconstruct past circulation. Any model treatment of $\epsilon_{\mathrm{Nd}}$ must not assume that $\epsilon_{\mathrm{Nd}}$ satisfies a simple advection-diffusion equation. Incorporation of this proxy in a model-data synthesis appears difficult at this point.

$\mathrm{Cd} / \mathrm{Ca}$ ratios of benthic foraminifera. The ratio of cadmium (Cd) to calcium $(\mathrm{Ca})$ in benthic foraminifera shells is an important proxy of past nutrient distributions [Marchitto and Broecker, 2006]. The $\mathrm{Cd} / \mathrm{Ca}$ ratio in foram shells $(\mathrm{Cd} / \mathrm{Ca}$ calcite $)$ reflects the $\mathrm{Cd} / \mathrm{Ca}$ ratio of the surrounding seawater $\left(\mathrm{Cd} / \mathrm{Ca}_{\text {water }}\right)$, with a partition coefficient $D_{\mathrm{Cd}}=$ $\left(\mathrm{Cd} / \mathrm{Ca}_{\text {calcite }}\right) /\left(\mathrm{Cd} / \mathrm{Ca}_{\text {water }}\right)$ that varies from a value of 2.9 at depths below $3000 \mathrm{~m}$ to 1.3 at depths above $1150 \mathrm{~m}$ [Marchitto and Broecker, 2006]. These partition coefficients are used to correct paleo records of $\mathrm{Cd} / \mathrm{Ca}$ for the depth effect before interpretation [Marchitto and Broecker, 2006].

Seawater cadmium concentrations follow a phosphate-like distribution, with low values in warm surface waters (caused by biological uptake of cadmium) and high values in deep waters (caused by remineralization of sinking organic material); the relationship between cadmium and phosphate is nearly linear with a slight curve that can likely be attributed to preferential uptake in organic matter of cadmium relative to phosphate [Marchitto and Broecker, 2006]. The net global ocean cadmium content can change over time due to changes in suboxic sediments (these are a primary sink of cadmium in the ocean); however, the oceanic residence time of cadmium is long, so that the total ocean $\mathrm{Cd}$ content at the LGM was likely similar to today [Boyle, 1988]. A key advantage of $\mathrm{Cd} / \mathrm{Ca}$ as a proxy of past nutrient distributions is that interspecies offsets in the uptake of $\mathrm{Cd} / \mathrm{Ca}$ are negligible [Boyle, 1988]. There are also disadvantages: cadmium is difficult to measure 
and subject to contamination, there may be inter-laboratory differences in cleaning procedures and calibration, and $\mathrm{Cd} / \mathrm{Ca}$ ratios are biased in regions bathed in undersaturated waters [Marchitto and Broecker, 2006]

Marchitto and Broecker [2006] compiled 75 cores with $\mathrm{LGM} \mathrm{Cd} / \mathrm{Ca}_{\text {calcite }}$ data; each core also included late Holocene or coretop $\mathrm{Cd} / \mathrm{Ca}_{\text {calcite }}$ measurements. Figure 2-9, a figure reproduced from Marchitto and Broecker [2006], shows Atlantic sections of inferred seawater cadmium distributions from both Holocene and LGM proxy records. As interpreted by Marchitto and Broecker [2006], inferred distributions of Holocene cadmium are consistent with modern nutrient distributions, with the exception of intermediate to mid-depth cores in the high latitude North Atlantic. In this region, inferred cadmium values are too high relative to modern nutrient distributions, the cause of which remains unknown. Inferred LGM cadmium distributions are substantially different from modern nutrient distributions, with evidence for low-nutrient Glacial North Atlantic Intermediate water (GNAIW) at shallower depths than modern NADW, and an increased presence of AABW in the North Atlantic [Marchitto and Broecker, 2006]; the transition between these water masses is inferred by Marchitto and Broecker [2006] to lie near 2200 to $2500 \mathrm{~m}$ water depth, in agreement with inferences based on Atlantic $\delta^{13} \mathrm{C}$ distributions for the LGM. A separate examination of cores from the eastern and western Atlantic by Marchitto and Broecker [2006] (not reproduced here) indicates that the LGM Atlantic may have been marked by reduced zonal gradients in cadmium below $3000 \mathrm{~m}$; this is additional evidence that the deep Atlantic was filled by a relatively uniform water mass of southern origin.

Evaluation for model-data syntheses. Marchitto and Broecker [2006] have compiled 75 Atlantic cores with LGM records of $\mathrm{Cd} / \mathrm{Ca}$, have included a detailed discussion of uncertainties, and have shown that spatially coherent patterns are present in Holocene and LGM $\mathrm{Cd} / \mathrm{Ca}$ records. Marchitto and Broecker [2006] also present straightforward mappings from $\mathrm{Cd} / \mathrm{Ca}_{\text {calcite }}$ to $\mathrm{Cd} / \mathrm{Ca}_{\text {water }}$, from which seawater nutrient distributions can be inferred. To incorporate these data in a model, seawater cadmium could be modeled and then compared with distributions inferred from data, or seawater phosphate could be modeled instead and 

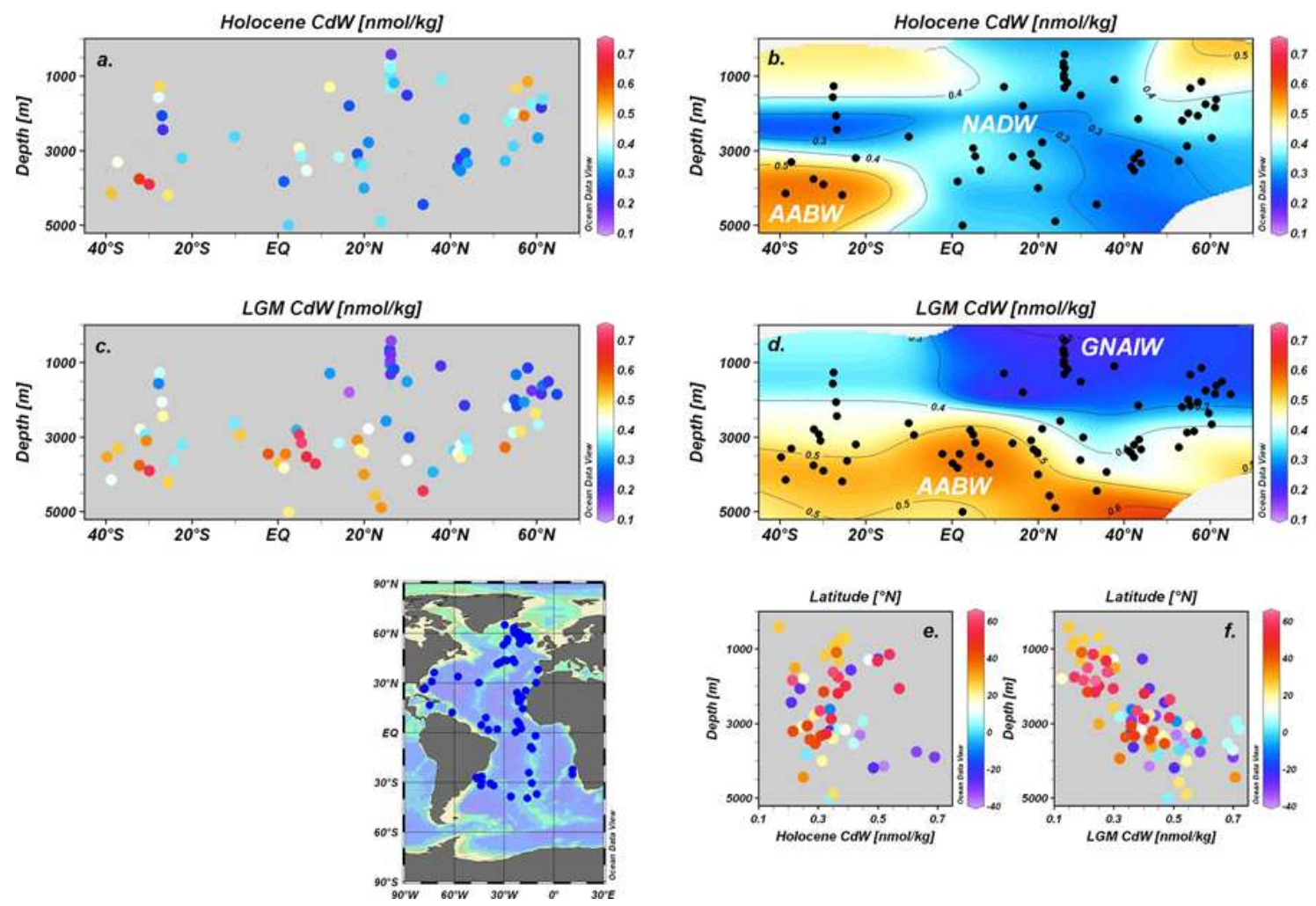

Figure 2-9: Seawater cadmium as inferred from benthic foraminiferal $\mathrm{Cd} / \mathrm{Ca}$ ratios for the Holocene (top row) and LGM (middle row) at core locations shown in bottom left map. Subplots (a) and (c) show individual records plotted against latitude, (b) and (d) show contour plots made with a strong smoothness requirement, and (e) and (f) show cadmium versus depth with colors to indicate latitude. Figure from Marchitto and Broecker [2006].

the modern $\mathrm{Cd} /$ phosphate relationship could be assumed to apply at the LGM. As an example, Winguth et al. [2000] applied an inverse approach to use $\mathrm{Cd} / \mathrm{Ca}$ proxy records to constrain LGM circulation (see Section 2.4.2 for details). Overall, with proper attention to uncertainties in paleo records of $\mathrm{Cd} / \mathrm{Ca}$, this proxy is a good choice for quantitative model-data comparisons and/or syntheses.

$\mathrm{Ba} / \mathrm{Ca}, \mathrm{Zn} / \mathrm{Ca}$ and $\mathrm{Mg} / \mathrm{Ca}$ ratios of benthic foraminifera. Like cadmium, barium $(\mathrm{Ba})$, zinc $(\mathrm{Zn})$ and magnesium $(\mathrm{Mg})$ substitute for calcium in $\mathrm{CaCO}_{3}$ shells. As was the case for cadmium, the rate at which barium and zinc are incorporated in foraminiferal shells is related to the barium and zinc content of the surrounding waters [Lynch-Stieglitz, 2003]; 
seawater barium and zinc concentrations largely follow nutrient distributions. The partition coefficient for the ratio of $\mathrm{Ba} / \mathrm{Ca}$ or $\mathrm{Zn} / \mathrm{Ca}$ in foraminifera to the ratio in seawater is highly dependent on saturation state, with partition coefficients potentially reduced by $50 \%$ or more in the abyssal Indo-Pacific [Marchitto and Broecker, 2006].

The $\mathrm{Mg} / \mathrm{Ca}$ ratio incorporated in benthic foraminifera is temperature dependent, just as it is for planktic foraminifera (see Section 2.2.2). Unfortunately, the $\mathrm{Mg} / \mathrm{Ca}$ paleothermometer is relatively insensitive at cold temperatures, so that uncertainties in the paleothermometer overwhelm the small dynamic range of deep ocean temperatures [Lynch-Stieglitz, 2003]. $\mathrm{Mg} / \mathrm{Ca}$ in benthic forams is most promising for reconstruction of temperatures at intermediate water depths where temperature variations are larger.

Evaluation for model-data syntheses. Although these proxies show potential, they are currently not well-positioned for quantitative model data synthesis. Compilations with significant numbers of proxy records do not yet exist, and uncertainties remain poorly quantified. The effort required to build a model of seawater zinc, barium, or magnesium may not be warranted until larger data sets are available.

Protactinium / thorium ratios. The ratio of ${ }^{231} \mathrm{~Pa}$ to ${ }^{230} \mathrm{Th}$ in sediments is a relatively new proxy that has shown promise for studies of ocean circulation [Mcmanus et al., 2004, Keigwin and Boyle, 2008, Burke et al., 2011]. Protactinium and thorium are radioisotopes that are both formed at a constant ratio of 0.093 from the decay of uranium [Mcmanus et al., 2004]. Thorium is more particle reactive and is thus scavenged from the water column more quickly (residence time of 20 - $40 \mathrm{yr}$ ), while protactinium persists longer in the water column (residence time of 100 - 200 years) [Mcmanus et al., 2004]. Because the Atlantic has a relatively vigorous flow of northern source waters, modern Atlantic sediments have a ${ }^{231} \mathrm{~Pa} /{ }^{230} \mathrm{Th}$ ratio that is significantly lower than 0.093 (the production rate). In the absence of any other changes, slower transport rates would result in higher ratios. For example, Mcmanus et al. [2004] observed ratios close to 0.093 during Heinrich Event 1 (H1, a period associated with extensive deposits of ice rafted detritus that occurred about 15-17 kyr bp) in North Atlantic cores and inferred that the AMOC was nearly or completely eliminated 
during this period.

Burke et al. [2011] compiled 236 measurements of modern water column dissolved or total ${ }^{231} \mathrm{~Pa}$ activity at 32 stations in the Atlantic as well as 39 sediment core records, each of which includes ${ }^{231} \mathrm{~Pa} /{ }^{230} \mathrm{Th}$ ratios for the Holocene, LGM, and $\mathrm{H} 1$ periods. Interpretation of ${ }^{231} \mathrm{~Pa} /{ }^{230} \mathrm{Th}$ ratios is complicated by several factors, which are summarized here; see Burke et al. [2011] for a thorough discussion. The ${ }^{231} \mathrm{~Pa} /{ }^{230} \mathrm{Th}$ ratio preserved in sediments is affected by the differential scavenging of the two elements, which depends not only on the rate at which particles are passing through the water column (the particle flux), but also on what types of particles are present (biogenic opal scavenges protactinium more readily than thorium, while for other particles the opposite is true) [Burke et al., 2011].

Evaluation for model-data syntheses. Burke et al. [2011] have compiled modern and paleo measurements related to ${ }^{231} \mathrm{~Pa} /{ }^{230} \mathrm{Th}$, have identified the key issues to be treated in a model of ${ }^{231} \mathrm{~Pa} /{ }^{230} \mathrm{Th}$, and have made formal uncertainty estimates. Other investigators interested in incorporating ${ }^{231} \mathrm{~Pa} /{ }^{230} \mathrm{Th}$ ratios in a model-data synthesis can base their approach on that developed by Burke et al. [2011].

Sediment properties as proxies for near bottom flow speed. It has been proposed that grain-size properties of deep-sea sediments can be used to reconstruct near-bottom flow speeds (see reviews in McCave and Hall 2006, McCave 2007). Sediment core samples are analyzed for grain-size distributions; smaller (larger) current speeds are inferred for finer (coarser) grain-sizes. Potential problems with this proxy include sediment source region changes, downslope supply on continental margins, the effect of flow over bedforms, and the influence of ice-rafted detritus on sediment properties [McCave and Hall, 2006]. The extent to which flow at the sediment water interface is representative of the flow of the overlying water mass is poorly constrained. Finally, there are few modern calibration studies to correlate grain-size properties with instrumental measurements of the flow speed.

McCave et al. [1995] and Manighetti and McCave [1995] use sediment properties to reconstruct flow speeds near Rockall Bank in the NE Atlantic Ocean. They find that production of Lower North Atlantic Deep Water (LNADW) was suppressed during the glacial 
and that intermediate waters generally had higher current speeds and may have been present over a larger depth domain (perhaps from $1100 \mathrm{~m}$ to more than $2000 \mathrm{~m}$ ). Covariation of records at 1100, 1750, and $2000 \mathrm{~m}$ suggest that one water mass may have bathed all three sites at the LGM. These authors correct for the grain size effect of ice-rafted detritus (IRD) by subtracting from each sediment record the size distribution from a site that is assumed to be unaffected by currents. Dating on most cores is done by correlation of properties with one core that is assumed to be accurately dated. Uncertainties associated with these data treatments are poorly constrained.

Evans and Hall [2008] reconstruct deepwater circulation on the Blake Outer Ridge (Western North Atlantic) during the Holocene, Younger Dryas, and Last Glacial Maximum. This study included 33 sediment cores collected in 3 depth transects. The authors infer a shift of the Deep Western Boundary Current (DWBC) core from 3000-4000 m in the Holocene to above $3000 \mathrm{~m}$ at the LGM, implying the presence of well-ventilated Glacial North Atlantic Intermediate Water (GNAIW), rather than Holocene North Atlantic Deep Water (NADW). At depths below 4000 m, Evans and Hall [2008] infer an increase in abyssal circulation and a robust presence of southern-sourced waters.

Evaluation for model-data syntheses. The availability of a bottom-water flow-speed proxy would be of great utility in reconstructing LGM circulation. The relative paucity of modern calibrations is the biggest hindrance to using these data. Nonetheless, these records could be incorporated as relative constraints: flow at site A is faster than flow at site B, or flow at site A at the LGM is faster than flow at site A at the Holocene. This type of constraint is straightforward to incorporate in model-data syntheses.

\subsection{Forward models of ocean state}

A large number of modeling studies over the past 30 years have focused on the LGM climate, with goals ranging from quantifying the impact of the ice sheets on atmospheric circulation (e.g., Manabe and Broccoli 1985), modeling the LGM dust cycle (e.g., Mahowald 
et al. 2006), and modeling the fully coupled ocean-atmosphere-sea ice system (e.g., Shin et al. 2003). The discussion here is limited to a few key studies that are particularly relevant to the thesis. The state of the art in coupled model simulations of the LGM is described in Section 2.3.1 and models of isotopes in the ocean are reviewed in Section 2.3.2. The studies described in these two sections are used later in the thesis as comparison points for the state estimates developed in the thesis.

\subsubsection{The Paleoclimate Modeling Intercomparison Project}

Although there have been many efforts to model the LGM climate, the Paleoclimate Modeling Intercomparison Project (PMIP) is the most coordinated, long-running, and extensive. PMIP's goal has been coordinated intercomparison and evaluation of paleoclimate models, with the long-term goal of improving climate models for better prediction of future climate. PMIP models use a consistent set of boundary condition definitions, permitting straightforward intercomparisons. We focus on PMIP here as the current consensus on LGM climate from the climate modeling community.

PMIP1, the first phase of PMIP, used atmosphere-only general circulation models with prescribed LGM sea surface temperatures or with single-layer slab ocean models [Joussaume and Taylor, 1995, Braconnot, 2000]. PMIP2 focused on coupled ocean-atmosphere and ocean-atmosphere-vegetation models [Braconnot et al., 2007a,b]. PMIP3 [Braconnot et al., 2012] is a relatively new effort that is coordinated with the fifth phase of the World Climate Research Program Coupled Model Intercomparison Project (CMIP5) [Taylor et al., 2012], as CMIP is now including paleoclimate simulations for the first time. In addition to continuing to model LGM and Holocene climate, PMIP3 is expanding its focus to additional time periods, particularly past warm climates, and to new aspects of the climate system, such as the carbon cycle [Braconnot et al., 2012]. Although a few LGM PMIP3 simulations are now available online, these results are very new and have not been fully evaluated by the PMIP3 community. Thus the following discussion focuses on PMIP2 simulations of LGM climate. 


\begin{tabular}{|l|l|l|l|l|}
\hline $\begin{array}{l}\text { Model name in } \\
\text { PMIP2 database }\end{array}$ & $\begin{array}{l}\text { Atm. Resol. } \\
\text { Lon x Lat }\end{array}$ & $\begin{array}{l}\text { Atm. } \\
\text { Levels }\end{array}$ & $\begin{array}{l}\text { Ocean Res. } \\
\text { Lon x Lat }\end{array}$ & $\begin{array}{l}\text { Ocean } \\
\text { Levels }\end{array}$ \\
\hline CCSM3 & $\mathrm{T} 42$ & 26 & $1^{\circ} \times 0.3-1^{\circ}$ & 40 \\
FGOALS-g1.0 & $2.8^{\circ} \times 2.8^{\circ}$ & 26 & $1^{\circ} \times 1^{\circ}$ & 33 \\
IPSL-CM4-V1-MR & $3.75^{\circ} \times 2.5^{\circ}$ & 19 & $2^{\circ} \times 1-2^{\circ}$ & 31 \\
MIROC3.2 & $\mathrm{T} 42$ & 20 & $1.4^{\circ} \times 0.5-1.4^{\circ}$ & 43 \\
HadCM3M2* $^{\circ}$ & $3.75^{\circ} \times 2.5^{\circ}$ & 19 & $1^{\circ} .25^{\circ} \times 1.25^{\circ}$ & 20 \\
ECBilt-Clio $^{\dagger}$ & $\mathrm{T} 21$ & 3 & $3^{\circ} \times 3^{\circ}$ & 20 \\
\hline
\end{tabular}

*Ocean-atmosphere-vegetation model ${ }^{\dagger}$ Intermediate complexity model

Table 2.2: Configurations used for PMIP2 LGM simulations. Spectral resolution T42 is about $2.8^{\circ} \times 2.8^{\circ}$; T21 is about $5.6^{\circ} \times 5.6^{\circ}$. Table follows Braconnot et al. [2007a] and Otto-Bliesner et al. [2007].

PMIP2 defined a standard set of LGM boundary conditions to be used by all modeling groups [Braconnot et al., 2007a]: ICE-5G ice sheets and coastlines [Peltier, 2004], atmospheric $\mathrm{CO}_{2}$ concentration of $185 \mathrm{ppmv}, \mathrm{CH}_{4}$ of $350 \mathrm{ppbv}, \mathrm{NO}_{2}$ of $200 \mathrm{ppbv}$, eccentricity of 0.018994 , obliquity of $22.949^{\circ}$, and angular precession of $114.42^{\circ}$. Changes in dust were neglected, resulting in a potential error in radiative forcing on the order of about $1 \mathrm{~W} / \mathrm{m}^{2}$ [Braconnot et al., 2007a]. The mean shift in LGM ocean salinity of about 1 or 1.1 was also neglected in the PMIP2 protocol (although one of the models, CCSM3, did incorporate a mean salinity change). In models without an active vegetation component, vegetation was set to modern-day conditions. Due to computational costs, it was not possible to require all modeling groups to follow a given protocol for a full spinup to quasi-equilibrium LGM-like ocean conditions; instead, each modeling group used their own approximate approach to spinup. Table 2.2 provides the configurations used for the PMIP2 LGM simulations that are discussed below. All six of these models have an interactive sea ice component. ECBilt-Clio used basin-mean flux adjustments; otherwise none of the models used flux adjustments.

Braconnot et al. [2007a] and Braconnot et al. [2007b] analyzed and compared all six simulations listed in Table 2.2; the rest of this paragraph refers to the results of these two related papers. PMIP2 models showed global mean temperature changes between the LGM 
and pre-industrial control runs from -3.6 to $-5.7{ }^{\circ} \mathrm{C}$, a relatively large spread indicating a wide range of modeled climate sensitivities. The PMIP2 ensemble mean showed cooling of $2-5^{\circ} \mathrm{C}$ over most of the low and mid-latitude Atlantic, with larger cooling of $5-10^{\circ} \mathrm{C}$ north of $45^{\circ} \mathrm{N}$ in the Atlantic. The ensemble mean also showed mostly large-scale drying, though the mid-latitude Atlantic ocean showed some regions with increased precipitation. LGM Atlantic sea ice is generally further south than in the pre-industrial simulations, though changes in seasonality of sea ice are model-dependent. The storm track activity shifts further south as well, partly in association with the sea ice shift and partly in association with a shift in SST gradients. Half of the Northern Hemisphere LGM cooling in PMIP2 models is due to feedbacks from mid to high latitude snow and sea ice, while reduced atmospheric $\mathrm{CO}_{2}$ and water vapor accounts for the remaining cooling [Braconnot et al., 2007b].

Otto-Bliesner et al. [2007] compare the pre-industrial (PI) and LGM Atlantic meridional overturning circulation (AMOC) in four PMIP2 models (ECBilt-CLIO, HadCM3M2, MIROC3.2.2, and CCSM3); the rest of this paragraph refers to results from this paper. The PI AMOC in these models varies from 13.8-20.8 Sv; all are somewhat too shallow relative to modern-day observations. In the LGM simulations, net AMOC transport is largely unchanged from the PI in HadCM and is weakened by about 20\% in CCSM3, while ECBilt and MIROC show increases of 20-40\%. Relative to the PI simulations, the LGM AMOC shoals significantly in CCSM3, shoals modestly in HadCM, and deepens significantly in MIROC and ECBilt. Otto-Bliesner et al. [2007] also compared deep ocean properties in the North Atlantic at Feni Drift (2184 m) to those in the South Atlantic at Shona Rise (3626 m). All four PMIP2 models simulate pre-industrial (PI) temperature and salinity (T/S) gradients between these two sites that are reasonable as compared to modern observations. However, the models strongly disagree with each other for LGM conditions; only CCSM3 simulates deep ocean LGM T/S gradients between the two sites that are similar to those reconstructed from pore fluid records.

Weber et al. [2007] focused on determining which processes control LGM AMOC 
structure in the same four PMIP2 models as Otto-Bliesner et al. [2007], as well as in the UVic ESCM2.7 model. These authors found that competition between the density of northern and southern water masses plays a key role, but that factors such as net evaporation and whether the PI control simulation was particularly cold or warm can also be important. The analyses of Weber et al. [2007] and Otto-Bliesner et al. [2007] indicate that, at least for the LGM, deep ocean circulation, deep water mass formation, and AMOC structure continue to be poorly understood and poorly constrained by climate models.

Otto-Bliesner et al. [2009] compare PMIP2 model results in the tropics $\left(15^{\circ} \mathrm{S}-15^{\circ} \mathrm{N}\right)$ to MARGO SSTs; all six models from Table 2.2 were used. The rest of this paragraph refers to results of this study. Unlike the other PMIP2 analyses described above, these authors compare to the individual MARGO proxy records, rather than the gridded MARGO SST product. The PMIP2 models find a tropical Atlantic cooling at the LGM of $1.5-2.6^{\circ} \mathrm{C}$, consistent with the MARGO estimate of $2.9 \pm 1.3^{\circ} \mathrm{C}$. The models show a cooling that is relatively uniform in space and time - there is little indication in the models of LGM shifts in meridional or zonal patterns or in seasonality. This result is in disagreement with the MARGO data, which indicate particularly strong LGM cooling in the eastern tropics, in the subpolar gyre, and in the winter season. Some tropical Atlantic processes remain difficult for these models to simulate correctly in the modern climate, making their LGM simulations difficult to interpret. Simulated PI SSTs are $2-4^{\circ} \mathrm{C}$ too warm in the upwelling regions along the western coast of Africa. All of the models also have difficulty correctly simulating upper ocean temperature structure in the equatorial region $\left(5^{\circ} \mathrm{S}\right.$ to $\left.5^{\circ} \mathrm{N}\right)$; as a result, the eastern equatorial Atlantic upper ocean temperatures are too high in all of the analyzed PMIP2 PI simulations.

\subsubsection{Isotope models}

Interpretation of $\delta^{13} \mathrm{C}_{\mathrm{DIC}}$ and $\delta^{18} \mathrm{O}_{\text {calcite }}$ proxy records, as will be done in this thesis, requires a model that can simulate (1) the physics of the ocean, atmosphere, and/or sea ice, as was done in PMIP2 and (2) the key controls on $\delta^{13} \mathrm{C}_{\text {DIC }}$ and $\delta^{18} \mathrm{O}_{\text {calcite }}$ signal recorded in 
proxies. This section reviews previous forward modeling efforts to simulate these isotopic signatures, with a particular focus on those approaches that have been tested in LGM simulations. It is beyond the scope here to provide a full description of all available approaches; instead, this section is designed to provide the interested reader with an overview of the current state of the art, and a starting place for further investigation. Note that only forward modeling studies are reviewed here; discussion of isotope modeling approaches used in previous model-data syntheses efforts in postponed to Section 2.4.2.

Oxygen isotopic ratios. Oxygen isotopes have been incorporated into several ocean circulation models such as the Geophysical Fluid Dynamics Laboratory Modular Ocean Model (GFDL MOM) [Paul et al., 1999], the CLIMBER-2 intermediate complexity Earth system model [Roche et al., 2004], the Goddard Institute for Space Studies (GISS) coupled ocean-atmosphere GCM [Schmidt et al., 2007], the Hadley Centre Climate model (HadCM3) coupled atmosphere-ocean GCM [Tindall et al., 2009], the NCAR Community Atmosphere Model (CAM3.0) [Sturm et al., 2010], and the University of Victoria Earth System Climate Model (UVic ESCM) [Brennan et al., 2011]. Note that such isotopes extensions may or may not become part of the primary model code (so they may not be available to use in new investigations). The two $\delta^{18} \mathrm{O}$ modeling studies that have included LGM-like scenarios are described below.

Roche et al. [2004] used the fluxes from the atmospheric GCM ECHAM to drive the intermediate complexity CLIMBER-2 model. The ocean component of CLIMBER-2 is a zonally averaged three basin model on a $2.5^{\circ}$ latitudinal grid, with 20 vertical levels. Oceanic $\delta^{18} \mathrm{O}_{\text {water }}$ is modeled as a passive tracer in the ocean component of CLIMBER-2, and a parameterization is developed to convert air-sea fluxes of water into air-sea fluxes of ${ }^{18} \mathrm{O} . \delta^{18} \mathrm{O}_{\text {water }}$ in a modern control simulation are compared to the Schmidt et al. [1999] database of modern $\delta^{18} \mathrm{O}_{\text {water }}$ measurements; most of the general trends in the modern observations are captured by the model, though this zonal mean, intermediate complexity model is not able to capture any east-west variation, and some details of the latitudinal variation are missed by the model as well [Roche et al., 2004]. For LGM simulations, the south 
to north gradient in deep ocean $\delta^{18} \mathrm{O}_{\text {water }}$ observed in pore fluid records (see Section 2.2.3) is reproduced in the model in terms of the sense of the gradient, but the CLIMBER-2 model does not produce a large enough LGM gradient. Roche et al. [2004] conclude that a larger $\mathrm{AABW}$ inflow to the Atlantic would be required to match the pore fluid records. Modeled $\delta^{18} \mathrm{O}_{\text {water }}$ and modeled temperature are also used to estimate modeled $\delta^{18} \mathrm{O}_{\text {calcite }}$ using a paleo temperature equation; modeled $\delta^{18} \mathrm{O}_{\text {calcite }}$ is then compared to Atlantic proxy records of LGM $\delta^{18} \mathrm{O}_{\text {calcite }}$. The deep ocean gradient in modeled $\delta^{18} \mathrm{O}_{\text {calcite }}$ is opposite of that seen in the data; based on the observation that the modeled deep ocean $\delta^{18} \mathrm{O}_{\text {water }}$ gradients are consistent with pore fluid records, Roche et al. [2004] conclude that the modeled deep ocean temperatures must not be an acceptable representation of LGM conditions.

In an unpublished discussion manuscript, Brennan et al. [2011] describe an implementation of oxygen isotopes in the UVic ESCM. The UVic ESCM is a fully coupled model that includes ocean, atmosphere, land surface, and sea ice, all simulated at a horizontal resolution of $3.6^{\circ}$ in longitude by $1.8^{\circ}$ in latitude; the ocean model has 19 vertical levels. The atmospheric model is simplified with vertically integrated equations for energy and moisture balance. Oceanic $\mathrm{H}_{2}^{16} \mathrm{O}$ and $\mathrm{H}_{2}^{18} \mathrm{O}$ are modeled as tracers which are influenced by processes such as runoff, evaporation and precipitation at the sea surface, but which are assumed conservative once water masses are isolated from air-sea exchange. Brennan et al. [2011] compare surface ocean $\delta^{18} \mathrm{O}_{\text {water }}$ in their modern simulation against the gridded $\delta^{18} \mathrm{O}_{\text {water }}$ estimate of LeGrande and Schmidt [2006]; the general trends in the two estimates are similar, though the model simulation does not sufficiently capture the range of observed $\delta^{18} \mathrm{O}_{\text {water }}$ values. For their simulation of LGM $\delta^{18} \mathrm{O}$, Brennan et al. [2011] compare the modeled $\delta^{18} \mathrm{O}_{\text {water }}$ of precipitation against a few proxy observations; the comparison is purely visual and there are few data, making it difficult to use the data to evaluate the skill of the LGM model estimate. No comparisons are made to LGM proxy records of oceanic $\delta^{18} \mathrm{O}$.

Carbon isotopic ratios. Hesse et al. [2011] focus on the impact of possible LGM changes in overturning circulation on oceanic $\delta^{13} \mathrm{C}$ distributions. Ocean circulation fields 
are generated with the Large Scale Geostrophic (LSG) Ocean Circulation Model (OGCM) and used to drive the HAMOCC2s marine carbon cycle model, which simulates chemical tracers in the ocean and the sediment (a 10 layer sediment model is included). Ocean resolution is $3.5^{\circ}$ in the horizontal with 22 vertical levels. All geochemical parameters used for both the modern and LGM simulations are set to preindustrial levels. There is no exploration of possible changes in surface ocean $\delta^{13} \mathrm{C}$ at the LGM; instead, the focus is on the potential effect of circulation changes. Several glacial simulations were developed by using two different LGM sea surface temperature reconstructions (CLIMAP and GLAMAP) and by using either standard Southern Ocean conditions or simulating increased brine release due to increased LGM export of sea ice from the Southern Ocean. Figure 2-10 compares the Hesse et al. [2011] modeled $\delta^{13} \mathrm{C}$ fields and their compilation of $220 \delta^{13} \mathrm{C}$ records; the experiments presented in this figure are as follows. "CS" uses CLIMAP SSTs and mimics increased brine release in the Southern Ocean associated with northward sea ice export; the resulting circulation has a weak (8 Sv) and very shallow (above $1000 \mathrm{~m}$ ) AMOC. "GB" uses GLAMAP SSTs and does not use increased brine release; the resulting circulation has an AMOC similar to today's (14 Sv and relatively deep). "GS" uses GLAMAP SSTs and increased increased Southern Ocean brine release; the resulting circulation has a $12 \mathrm{~Sv}$ AMOC that is shallow $(2000 \mathrm{~m})$. Although the CS and GS model estimates show similar trends to the data, many points lie outside the included $\pm 0.5 \%$ lines.

A series of papers have studied modern to LGM $\delta^{13} \mathrm{C}$ changes in the ocean and atmosphere using the CLIMBER-2 intermediate complexity model [Brovkin et al., 2002b, 2007, Bouttes et al., 2011]. This model includes an atmospheric model with a $10^{\circ}$ latitudinal resolution and a basin-scale, $51^{\circ}$ longitudinal resolution; a zonally averaged, 3-basin ocean model with 20 vertical levels; a simple sea ice parameterization; a terrestrial biosphere model; and an ocean biogeochemistry model [Brovkin et al., 2007]. The carbon cycle model and treatment of isotopes is described in Brovkin et al. [2002a]. This computationally efficient model has been applied to study the potential contribution of changes in the ocean biological carbon pump, physical circulation changes, and $\mathrm{CaCO}_{3}$ system changes 

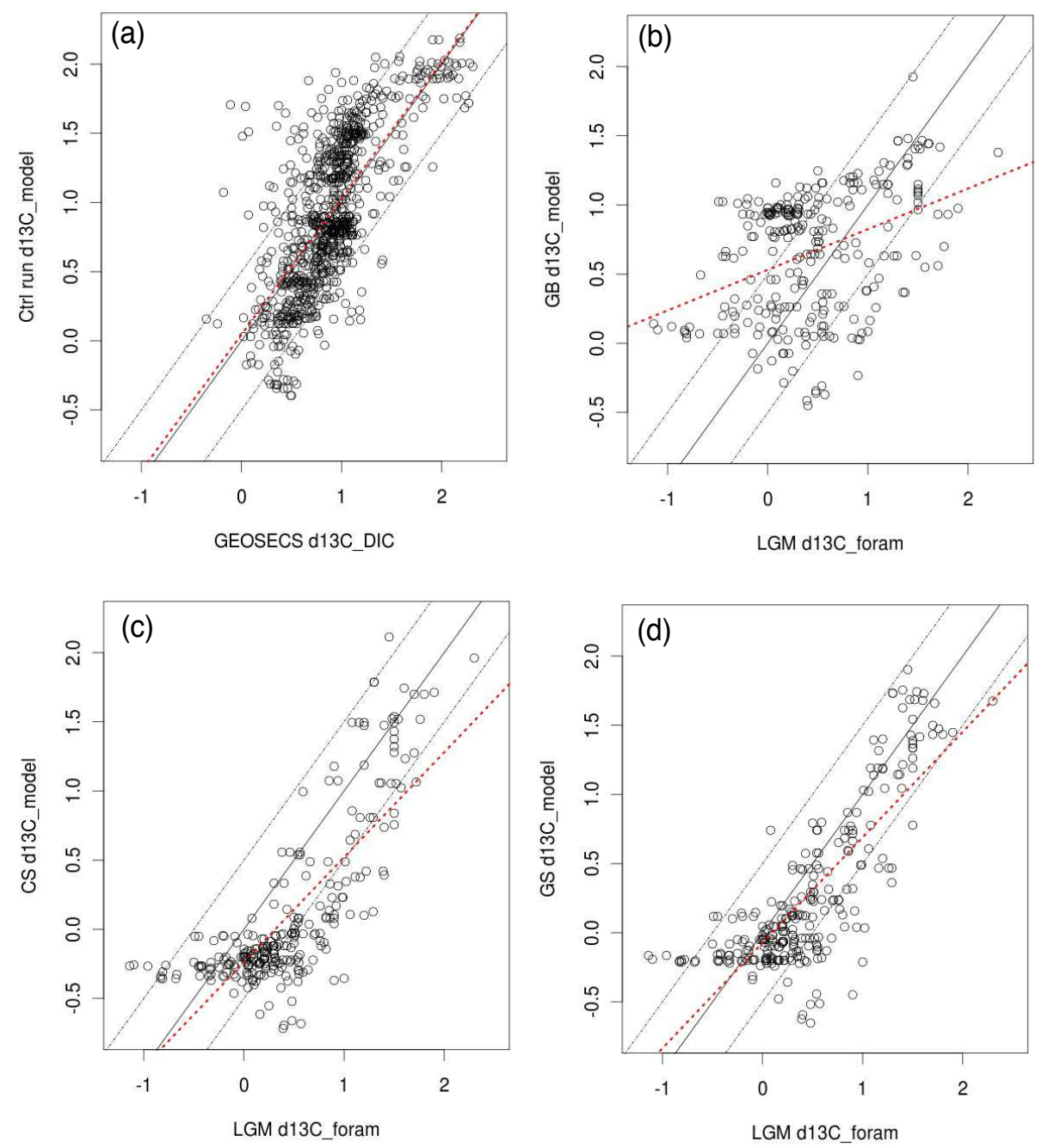

Figure 2-10: Scatter plots of modeled versus observed $\delta^{13} \mathrm{C}$ for four scenarios modeled by Hesse et al. [2011]; all axes are in \%o, the central lines show a one-to-one match, and dashed lines mark $\pm 0.5 \%$ ranges. (a) Modern control scenario compared to GEOSECS $\delta^{13}$ C. (b)(d) Three LGM scenarios compared to measured $\delta^{13} \mathrm{C}_{\text {calcite }}$ from benthic foraminifera. See the text for description of the three LGM simulations. Figure after Hesse et al. [2011], supplementary.

to glacial $\mathrm{CO}_{2}$ drawdown [Brovkin et al., 2002b, 2007, Bouttes et al., 2011]. The most direct model-data comparisons for $\delta^{13} \mathrm{C}$ are made in Brovkin et al. [2007] (their Figure 9), in which Atlantic sections of modeled and observed modern and LGM $\delta^{13} \mathrm{C}$ distributions can be visually compared. Quantitative model-data comparisons are not given; indeed, with a highly parameterized, zonal-mean three basin model of ocean circulation, this modeling 
approach is better suited to exploration of a wide parameter space and sensitivity studies than to truthfully reproducing observed characteristics of the ocean.

Tagliabue and Bopp [2008] and Tagliabue et al. [2009] employ the ORCA2 ocean GCM with a dynamic-thermodynamic sea ice model at $2^{\circ} \times 2^{\circ}$ resolution with 30 vertical levels; the global model is driven by climatological atmospheric forcing. Ocean biogeochemistry is simulated using PISCES - an ocean ecosystem model that simulates a variety of plankton types as well as the ocean carbon cycle and nutrient distributions. The concentration of ${ }^{13} \mathrm{C}$ is simulated in 10 distinct carbon pools, and a parameterization is used to simulate fractionation during photosynthesis, calcite precipitation, gas exchange, and carbonate chemistry (see Tagliabue and Bopp 2008 and references therein for a full description). Tagliabue and Bopp [2008] focus on the variability in modern ocean ${ }^{13} \mathrm{C}$ over the period of 1860-2000, and compare modeled $\delta^{13} \mathrm{C}$ against observations compiled by Gruber et al. [1999]; comparisons of model and data are largely qualitative, though correlation coefficients are reported for the fit of model to data (the model estimate which best reproduces the data has an $\mathrm{R}$ value of 0.86). In Tagliabue et al. [2009], a variety of LGM circulation scenarios are explored and the resulting $\delta^{13} \mathrm{C}$ distributions are compared against 133 records compiled by the Models and Observations to Test clImate Feedbacks project (MOTIF, http:motif.lsce.ipsl.fr). The LGM circulation scenario that best matches the data has an AMOC transport of $5 \mathrm{~Sv}$, an AMOC depth of $1750 \mathrm{~m}$ (the depth at which transport is zero), and an AABW transport of $4 \mathrm{~Sv}$. This circulation scenario gives a $\delta^{13} \mathrm{C}$ distribution that has a correlation of 0.6 with the MOTIF database, though the slope (0.31) and intercept $(-0.19 \%)$ of the fit indicate biases in the estimated $\delta^{13} \mathrm{C}$ distribution.

\subsection{Using models and data together to study the LGM}

Two methods of using models and data together to study the LGM Atlantic Ocean are described in this section: making model-data comparisons that are quantitative, rather than qualitative, in nature (Section 2.4.1); and building model-data syntheses (Section 2.4.2). 


\subsubsection{Quantitative model-data comparison}

With the long traditions of forward simulation of LGM conditions and the extensive efforts to collect and compile proxy records, quantitative model-data comparison is logical. However, most efforts to consider both model results and paleo data reconstructions have focused on visual comparisons; for example, this is true of most of the studies described in Sections 2.3.1 and 2.3.2). This section focuses on efforts to make comparisons of LGM models and data that are more quantitative in nature.

Kohfeld and Harrison [2000] presented an early effort to collect and describe compilations of diverse proxies for LGM and mid-Holocene climate and to compare these compilations to a variety of climate model results; this paper noted many of the key issues that still face the data and modeling communities in trying to move beyond qualitative comparisons. Building on the analyses of Kohfeld and Harrison [2000], Braconnot et al. [2012] (see especially the supplementary information) present updated and expanded LGM and Mid-Holocene data compilations that can be used in CMIP5/PMIP3 for quantitative model evaluation. These compilations include reconstructions of lake levels, vegetation patterns, biomass burning, dust deposition, terrestrial temperatures, and the MARGO NSST proxies. It is expected that the PMIP3/CMIP5 models will be systematically evaluated using these compilations. Although many difficulties remain, these efforts are promising collaborations between the data and modeling communities.

Hargreaves et al. [2011] present a quantitative comparison of PMIP1 and 2 model results with the gridded MARGO NSST estimate from Waelbroeck et al. [2009]. Because PMIP1 models did not have full ocean models we summarize only the PMIP2-related results. The reliability of the PMIP2 ensemble was evaluated against the MARGO data using techniques developed in the forecasting and modern climate model evaluation communities [Hargreaves et al., 2011]. As defined by Hargreaves et al. [2011], a "reliable" ensemble is one in which an observation is equally likely to be close to any given ensemble member. Consider, for example, the MARGO NSST estimate at a given geographic location and the ensemble of model estimates of NSST at that location. In a "reliable" ensemble, 
two characteristics are required: (1) the range of model estimates should, in general, encompass the observation, and (2) there should be an equal likelihood that any given model provides an NSST estimate that is closest to the observation. If MARGO uncertainties are neglected in the ensemble analysis, the PMIP2 ensemble is found to be too narrow - at many data locations, the MARGO NSST estimate is either warmer or colder than all of the model estimates. In a second test, the PMIP2 model results are modified by adding randomly generated perturbations equivalent to the MARGO error estimates. In this case, the ensemble is found to be "reliable", according to the definition of Hargreaves et al. [2011]. Regional patterns are apparent in the ensemble/data comparisons: the models tend to be too cold in the Greenland, Iceland and Norwegian Seas, too warm south of Iceland, too cold in the western low-latitude Atlantic, and too warm in the eastern low-latitude Atlantic. Hargreaves et al. [2011] did not test whether their evaluation approach could successfully reject model ensembles that are not expected to be good estimates of LGM conditions (a perturbed pre-industrial ensemble would have provided a useful check).

\subsubsection{Synthesizing models and data}

This section describes selected previous studies that built model-data syntheses for the LGM; the focus is on studies that employed real observations, rather than synthetic data.

LeGrand and Wunsch [1995] employed a simplified representation of the deep North Atlantic (from $1 \mathrm{~km}$ depth to the bottom at $1 \mathrm{~km}$ vertical resolution and from $10^{\circ} \mathrm{N}$ to $50^{\circ} \mathrm{N}$ at $10^{\circ}$ horizontal resolution). Ocean physics was described with linear vorticity and thermal wind balances, conservation of mass, potential temperature, salinity, and $\delta^{13} \mathrm{C}$. LGM records of $\delta^{18} \mathrm{O}_{\text {calcite }}$ and $\delta^{13} \mathrm{C}_{\text {calcite }}$ were shown to be consistent both with the modern circulation and with a circulation with NADW transport reduced by half from the modern.

The study of Winguth et al. [1999] is the only previous application of the adjoint technique to reconstruct LGM circulation. Winguth et al. [1999] used the Hamburg large-scale geostrophic ocean circulation model at approximately $3.5^{\circ} \times 3.5^{\circ}$ horizontal resolution with 11 vertical levels; a simple thermodynamic ice model was included, and the model was 
driven by relaxation to prescribed air temperatures and salinities. Modeled $\delta^{13} \mathrm{C}$ and $\mathrm{Cd} / \mathrm{Ca}$ were computed diagnostically from the phosphate distribution, and were constrained with benthic $\delta^{13} \mathrm{C}_{\text {calcite }}$ and $\mathrm{Cd} / \mathrm{Ca}$ data (uncertainties of $0.2 \%$ were applied for the $\delta^{13} \mathrm{C}_{\text {calcite }}$ data). Winguth et al. [1999] employed a single type of control variable: the surface salinity distribution to which the model was relaxed. Winguth et al. [1999] found that deep sea $\delta^{13} \mathrm{C}$ and $\mathrm{Cd} / \mathrm{Ca}$ data are consistent with a North Component Water transport that is reduced in magnitude by $50 \%$ and that is shallower than modern. The adjusted surface salinity field shows a strong positive freshwater anomaly in the North Atlantic.

Gebbie and Huybers [2006] applied a steady state geostrophic inverse model to reconstruct LGM meridional circulation at $30^{\circ} \mathrm{S}$ from South Atlantic $\delta^{18} \mathrm{O}$ measurements by Lynch-Stieglitz et al. [2006]. A $1^{\circ}$ horizontal resolution and $120 \mathrm{~m}$ vertical resolution were used, and model variables included the two dimensional fields of temperature, salinity, and meridional velocity. $\delta^{18} \mathrm{O}_{\text {calcite }}$ was computed as a linear combination of salinity and temperature, with empirical relationships used to determine the temperature and salinity dependencies. Holocene $\delta^{18} \mathrm{O}_{\text {calcite }}$ proxy records were shown to be consistent with a modern circulation estimate. LGM $\delta^{18} \mathrm{O}_{\text {calcite }}$ proxy records were then shown to also be consistent with the modern circulation, assuming an uncertainty of $0.2 \%$ on the data. Indeed, Gebbie and Huybers [2006] show that the data require a circulation different from today's only if three assumptions are made: temperature and salinity distributions are spatially smooth, the dependence of $\delta^{18} \mathrm{O}_{\text {water }}$ on salinity is linear and spatially invariant, and LGM temperatures along the margins at $30^{\circ} \mathrm{S}$ are known within $1^{\circ} \mathrm{C}$.

Marchal and Curry [2008] applied a 3-dimensional inverse model to the abyssal Atlantic circulation in conjunction with $180 \mathrm{LGM} \delta^{18} \mathrm{O}_{\text {calcite }}$ and $\delta^{13} \mathrm{C}_{\text {calcite }}$ proxy records. The model used a domain of $46^{\circ} \mathrm{S}$ to $64^{\circ} \mathrm{N}$ at $2^{\circ}$ resolution and covered depths of $1000-4750 \mathrm{~m}$ with three vertical levels. Ocean physics include hydrostatic and geostrophic balance, volume conservation, and a vertical diffusion term. $\delta^{18} \mathrm{O}$ and $\delta^{13} \mathrm{C}$ are represented as passive tracers with a simple approach to remineralization for $\delta^{13} \mathrm{C}$. Marchal and Curry [2008] found that more than $80 \%$ of LGM proxy records of $\delta^{18} \mathrm{O}_{\text {calcite }}$ and $\delta^{13} \mathrm{C}_{\text {calcite }}$ are consistent with the 
modern circulation, given assumed uncertainties of 0.1 to $0.2 \%$ on the isotope records. $\delta^{13} \mathrm{C}_{\text {calcite }}$ records could be shown to require a circulation different from the modern by including a stricter assumption: that deep ocean $\delta^{13} \mathrm{C}_{\mathrm{DIC}}$ at the LGM was dominated by advection and remineralization (i.e., that mixing had a negligible influence on the $\delta^{13} \mathrm{C}_{\mathrm{DIC}}$ distribution).

Burke et al. [2011] used a $4^{\circ} \times 4^{\circ}$ horizontal resolution inverse model of the abyssal Atlantic to test if LGM, H1, and/or Holocene ${ }^{231} \mathrm{~Pa} /{ }^{230} \mathrm{Th}$ proxy records require a circulation different from the modern one; the model used was similar to that used by Marchal and Curry [2008]. The authors showed that most modern water column ${ }^{231} \mathrm{~Pa}$ measurements are consistent with the modern circulation. These authors then showed that very few of the Holocene, LGM, or $\mathrm{H} 1{ }^{231} \mathrm{~Pa} /{ }^{230} \mathrm{Th}$ proxy records had to be adjusted by more than $2 \sigma$ to reach consistency with the modern circulation, indicating that these data are not sufficient to require a circulation different from today's. It was, however, possible to reject a state of no flow based on the modern, LGM, and H1 data.

A new and as yet unpublished inversion of LGM Atlantic Ocean proxy data by G. Gebbie (pers. comm.) is relevant to this thesis. The study used Total Matrix Intercomparison, a steady state inversion approach designed for the study of water mass decompositions and the transport pathways connecting the interior ocean with the surface [Gebbie and Huybers, 2010]. For study of the LGM, a $4^{\circ} \times 4^{\circ}$ horizontal resolution with 33 vertical levels was used. Modern circulation was estimated by inversion of modern $\delta^{18} \mathrm{O}_{\text {water }}$ and $\delta^{13} \mathrm{C}_{\text {DIC }}$ data as well as WOCE climatology of temperature, salinity, phosphate, nitrate, and oxygen. LGM data considered by the study include the gridded MARGO NSST estimate, porewater measurements, and benthic records of $\delta^{18} \mathrm{O}_{\text {calcite }}, \delta^{13} \mathrm{C}_{\text {calcite, }}$, and $\mathrm{Cd} / \mathrm{Ca}$. A key result found by Gebbie is that the LGM $\delta^{13} \mathrm{C}_{\text {calcite }}$ data are not consistent with the modern circulation. A limitation of the study is that dynamical constraints are limited to requirements of mass conservation and gravitational stability. 


\subsection{Selection of data constraints for the thesis}

Table 2.1 provided an advance preview of the data used in the thesis to constrain our ocean state estimates. These data were chosen because compilations were readily available and included substantial numbers of records located in the Atlantic Ocean, uncertainty estimates had been made, we were able to identify clear approaches to including the data in a model-data synthesis, and the selected data had been previously interpreted as showing significant LGM changes. There are a number of other datasets that would have been relatively straightforward to include, particularly $\mathrm{Cd} / \mathrm{Ca}$, sea ice estimates, and pore fluid records. With a greater investment of time and effort, radiocarbon and protactinium/thorium could have been incorporated as well; these proxies have the potential to constrain rates and timescales. In an ideal world, a single ocean state estimate could be developed that includes all of these proxies, as well as others. Such an effort was outside the scope of the thesis.

\subsection{Chapter summary}

This chapter has provided an overall picture of the LGM Atlantic with an emphasis on previous results of relevance to the rest of the thesis, and on providing the interested reader with a reference-rich starting point for further investigation. Upper and deep ocean proxies of ocean state were reviewed, and our judgement was given as to the difficulty with which each type of proxy could be included in a model-data synthesis. Previous forward modeling studies of the LGM coupled climate state were described, with an emphasis on PMIP. Models of LGM $\delta^{18} \mathrm{O}$ and $\delta^{13} \mathrm{C}$ were also described. Quantitative model-data comparison and synthesis are key topics of this thesis; particular attention was given in this chapter to previous efforts in these areas. 


\section{Chapter 3}

\section{State estimation methodology with application to modern circulation}

Adjoint-based ocean state estimation has been applied to reconstruct paleo ocean circulation in only one previous study [Winguth et al., 2000]. As described in Section 2.4.2, that study demonstrated the feasibility of the approach, but was limited in a number of important ways. Given the paucity of previous applications of adjoint models to paleo circulations, an appropriate ocean state estimation setup is not available. In this chapter, a new framework appropriate to paleo ocean state estimation is presented, and the utility of this framework is explored in the context of the modern ocean circulation - a system that is much better observed and understood than the LGM ocean. In testing these approaches in the modern context, the goal is to obtain a physically acceptable and interpretable solution that is similar to the modern ocean state. The modern state estimates presented here are case studies, useful for thinking about approaches to paleo ocean state estimation, but are not designed to answer problems of modern oceanography.

This chapter is organized as follows. Section 3.1 describes the numerical model of ocean circulation and its configuration (the forward model description). Section 3.2 describes how we apply a least-squares formulation to improve the fit of the model to observations (the inverse model description). Section 3.3 presents a modern ocean state estimate 
that is constrained by modern temperatures and salinities. In Section 3.4, extensions to the model for simulation of $\delta^{18} \mathrm{O}$ and $\delta^{13} \mathrm{C}$ are presented; these extensions are then applied to build a state estimate that is constrained by modern hydrography as well as modern water column measurements of $\delta^{13} \mathrm{C}$ and $\delta^{18} \mathrm{O}$. Section 3.5 describes new techniques for the application of state estimation to seasonal steady state problems. Finally, Section 3.6 provides a summary of novel contributions in the chapter, and discusses remaining limitations in state estimation tools for climate research.

\subsection{Forward model configuration}

The model configuration is described in two sections: the numerics, domain, and bathymetry are described in Section 3.1.1, while the choice of first guess initial, southern boundary, and atmospheric forcing are described in Section 3.1.2.

\subsubsection{Numerics and domain}

The MIT General Circulation Model [Marshall et al., 1997, Adcroft et al., 2004] is used to simulate ocean circulation under hydrostatic, Boussinesq, and linear implicit free surface assumptions. The model is under active development and is constantly changing; up-todate information and the code itself are available at http://mitgcm.org. The ocean model setup uses third order advection with direct space time treatment, and vertical diffusive fluxes are treated implicitly. A $1^{\circ} \times 1^{\circ}$ horizontal resolution latitude-longitude Arakawa Cgrid [Arakawa and Lamb, 1977] is used. Since eddies are not resolved at $1^{\circ}$ horizontal resolution, eddy-induced mixing of properties along isentropes is parameterized with GM/Redi: the Redi scheme applies a diffusion operator to mix properties along isentropes [Redi, 1982] and the GM scheme applies an advective flux to parameterize the transport effect of eddies [Gent and McWilliams, 1990]. A turbulent kinetic-energy based model is used to parameterize vertical mixing (GGL, Gaspar et al. [1990]). Table 3.1 lists model parameters used here. Side (bottom) boundary conditions are free-slip (no-slip). GCMs applied at 
a low resolution, such as is used here, suffer from gridscale noise associated with spatial averaging of the Coriolis terms. The C-D scheme [Adcroft et al., 1998] is applied here to address this issue by carrying a second set of velocity variables computed on a different grid (the D grid) in addition to those computed on MIT GCM's C grid.

\begin{tabular}{|lll|}
\hline Lateral diffusivity & $10^{2}$ & $\mathrm{~m}^{2} / \mathrm{s}$ \\
Vertical diffusivity & $10^{-5}$ & $\mathrm{~m}^{2} / \mathrm{s}$ \\
Laplacian lateral viscosity & $10^{4}$ & $\mathrm{~m}^{2} / \mathrm{s}$ \\
Laplacian vertical viscosity & $10^{-3}$ & $\mathrm{~m}^{2} / \mathrm{s}$ \\
GM thickness diffusivity & $10^{3}$ & $\mathrm{~m}^{2} / \mathrm{s}$ \\
Quadratic bottom drag & 0.002 & \\
\hline
\end{tabular}

Table 3.1: Model parameters used in all state estimates.

Sea ice is modeled following Losch et al. [2010]. Air-sea boundary conditions are prescribed for air temperature, $\mathrm{u} / \mathrm{v}$ wind speeds, relative humidity, precipitation, runoff, and incoming shortwave/longwave radiation. Bulk formulae are used to compute wind stress, evaporation and outgoing longwave radiation following Large and Yeager [2004]. In the standard configuration, momentum and tracer equations are advanced at the same timestep of 50 minutes (synchronous timestepping); an alternative asynchronous timestepping approach is described and tested in Section 3.5.

Later in this chapter the modern OCean Comprehensible Atlas (OCCA) of ocean properties over 2004-2006 [Forget, 2010] is employed as a surrogate for modern hydrographic data. While this estimate should not be regarded as "truth", it is a useful estimate of ocean properties that is consistent with a numerical model of ocean circulation and with a large number of observations, especially ARGO float profiles that have become available since the mid-2000s. To simplify comparisons of ocean state estimates made here and the OCCA estimate, the OCCA configuration is adopted where possible. A regional subset of the OCCA bathymetry and of the OCCA $1^{\circ} \times 1^{\circ}$ resolution model grid is used that extends from $40^{\circ} \mathrm{S}$ to $80^{\circ} \mathrm{N}$ and from $99^{\circ} \mathrm{W}$ to $21^{\circ} \mathrm{E}$. As in OCCA, there are 50 levels in the vertical, ranging in thickness from $10 \mathrm{~m}$ near the surface to $450 \mathrm{~m}$ near the bottom. The influence 
of inflow and outflow through the $40^{\circ} \mathrm{S}$ southern boundary of the model is represented by prescribing temperatures, salinities, and horizonal velocities at all longitudes and all depths of the section across $40^{\circ} \mathrm{S}$. A model boundary with prescribed conditions such as this one is called an "open boundary" and the prescribed conditions are called "open boundary conditions" (open boundary condition numerics are described by Zhang and Marotzke 1999); the specific properties we prescribe are described in Section 3.1.2.

Interior ocean properties are gradually relaxed to prescribed boundary condition properties over a sponge layer that extends from $36^{\circ} \mathrm{S}-40^{\circ} \mathrm{S}$; the relaxation timescale is linearly increased from $1 / 2$ day at $40^{\circ} \mathrm{S}$ to 10 days at $36^{\circ} \mathrm{S}$. This approach reduces discontinuities and wave generation associated with the boundary. Although there are important transports to and from the Arctic at $80^{\circ} \mathrm{N}$ in the modern Atlantic, the details of the exchange are uncertain. Available ocean state estimates, such as OCCA and ECCO V3 [Wunsch et al., 2009] do not include the Arctic and thus cannot be used as a reasonable first estimate of boundary conditions at $80^{\circ} \mathrm{N}$. The LGM exchange is particularly uncertain as the Bering Straits were closed at that time. A wall is thus imposed at $80^{\circ} \mathrm{N}$. This choice is likely to lead to overly warm and salty conditions in the northern portion of our domain since the normal exchange of warm salty Atlantic waters with cold, fresh Arctic waters is prevented; inflow from the Arctic to the Atlantic in the East Greenland Current is also a source of sea ice to the Northern North Atlantic, so the imposition of a wall may interfere with the model's capacity to produce enough sea ice in this region. Due to the relatively unphysical nature of the model configuration near the northern and southern boundaries, all simulations are run on the $40^{\circ} \mathrm{S}-80^{\circ} \mathrm{N}$ domain, but all analyses and figures are restricted to $35^{\circ} \mathrm{S}$ and $75^{\circ} \mathrm{N}$. It is worth remembering that, especially over long time periods, the influence that remote boundary conditions have on the interior is not well-understood, even for the modern circulation.

The configuration of the MIT GCM used here is conventional; MIT GCM model configurations with numerical schemes and resolution similar to that used here have been shown to provide an adequate representation of a wide variety of modern ocean regions and pro- 
cesses. The interested reader is referred to the OCCA estimate of 2004-2006 global ocean state [Forget, 2010] and the ECCO V3 estimate of 1992-2007 global ocean state [Wunsch et al., 2009], both of which were computed on a latitude-longitude grid at $1^{\circ}$ resolution, as is used here. The ECCO state estimates have been heavily analyzed to study many modern processes, including, for example, analysis of Atlantic meridional overturning and heat flux [Wunsch and Heimbach, 2006], the time required to reach tracer equilibrium in the deep ocean [Wunsch and Heimbach, 2008], and seasonal and decadal variability [Wunsch and Heimbach, 2009]. In summary, model setups similar to those used in this thesis, including mixing parameterizations, model resolution, air-sea bulk formulae, mixed layer scheme and Ekman layer representations, have proven sufficient for investigating a variety of problems in modern oceanography.

\subsubsection{Initial and boundary conditions}

An initial ocean circulation estimate is required before proceeding with improving the fit of the model to observations. First guess initial and boundary conditions are imposed and the forward model, described in the previous section, is used to make the initial circulation estimate. The first guess initial and boundary conditions used for this process are as follows.

The model is initialized with the OCCA estimate of January temperatures and salinities. Open southern boundary conditions are given by monthly mean OCCA properties at $40^{\circ} \mathrm{S}$; prescribed variables are temperature, salinity, and zonal and meridional velocities. In a simplified representation of the modern ocean, as is used here, a seemingly logical choice for atmospheric forcing is a monthly mean over many years of modern atmospheric forcing. In practice, averaging atmospheric conditions over long time periods leads to low-energy, overly smooth atmospheric properties. A specific year of modern atmospheric forcing is thus a more appropriate first guess. Following OCCA, the NCEP/NCAR reanalysis product is used (an updated version of Kalnay and coauthors [1996]); while OCCA uses 6-hourly forcing, daily mean forcing is employed here as this choice is required for asynchronous timestepping, an approach to be described and applied in Section 3.5. In using daily mean 
forcing, it is assumed that the diurnal cycle is not of first order importance in setting the large scale properties of the Atlantic Ocean.

Atmospheric forcing from 2006 was chosen arbitrarily from the three years estimated by OCCA. Given that these fields are adjusted to build a state estimate, the choice of year is not expected to be important. Indeed, analysis of adjustments made in modern ocean state estimates [Stammer et al., 2004] has shown that the adjusted fields (e.g. the wind field) can be more consistent with observations (e.g. satellite wind field observations) than was the first guess (an estimate from a reanalysis product ${ }^{1}$ Nonetheless, given sufficient resources, the state estimation process should be repeated with several different first guess estimates (e.g. different years of NCEP/NCAR and other reanalysis products such as Dee et al. 2011 or Griffies et al. 2009).

\subsection{Ocean state estimation}

The previous section defined a regional, forward model for simulating Atlantic Ocean circulation. An unconstrained ocean model such as this forward model will not generally be in good agreement with the target observations, making it difficult to draw links between the results of numerical studies and the real ocean. Many factors contribute to disagreement between ocean models and ocean observations; in addition to biases and uncertainties in the observational record, there are errors in the model initial and boundary conditions, errors introduced through the parameterization of subgrid-scale processes, errors in the numerical method of solution, and missing physics.

Ocean state estimation permits the formal treatment of these uncertainties in the search for a model-data synthesis that agrees with both the observations and the model, given their respective estimated uncertainties. An overview of the approach is presented below, but the technique description is kept brief as extensive overviews are available in the references.

\footnotetext{
${ }^{1}$ Reanalyses products such as the NCEP/NCAR reanalysis are produced by assimilating data from an extended period of time into a single numerical model or analysis system.
} 


\subsubsection{A least-squares problem and the method of Lagrange multipli- ers}

We seek an "ocean state estimate": an estimate of the modern ocean circulation that is consistent with known oceanic physics, as described by a numerical ocean circulation model, and with available observations of the modern ocean. A consistent solution is one in which the modeled ocean state is within uncertainty of observations. External boundary conditions in the model, including atmospheric forcing and initial conditions, are assumed to be the most uncertain aspects of the model. These boundary conditions are treated as control variables, meaning they can be adjusted to provide an improved fit of the modeled ocean state to the observations. Prior uncertainty estimates on control variables are accounted for by penalizing control variable adjustments; the goal is an improved estimate of control variables where the adjusted controls remain within the prior uncertainty of the initial estimate. To make progress, simplifying assumptions must be made. All variables are assumed to be Gaussian and all reported uncertainties represent one standard deviation $(1 \sigma)$. Uncertainties do not incorporate any estimates of covariance in space or time - all data and control variables are assumed to be independent.

This problem is formalized as a large model-observation least-squares minimization. The goal is to minimize a cost function

$$
J=J_{d a t a}+J_{c t r l}
$$

where $J_{\text {data }}$ is the cost of model-data misfit and $J_{c t r l}$ is the penalty on control variable adjustments. The general structure of $J_{d a t a}$ and $J_{c t r l}$ is presented here; full details are given in Sections 3.2.2 and 3.2.3. This thesis presents a number of different ocean state estimates; as needed, modifications to $J_{d a t a}$ and $J_{c t r l}$ are included in the methods specific to each estimate. The goodness of model-data fit is given by

$$
J_{\text {data }}=\sum(\text { model }- \text { data })^{T} \mathbf{W}_{\text {data }}(\text { model }- \text { data })
$$


where bold face is used to denote vectors or matrices, the sum is taken over all observations, data are observations arranged as a vector, model are the modeled values of those observation, and $\mathbf{W}_{\text {data }}$ is a weighting matrix that controls the relative contribution of each observation to the cost function. As shown by Lorenc [1986], the minimum variance solution is obtained when the weight matrix is the inverse of the noise covariance matrix; if the error statistics are jointly normal, this choice of weights also leads to the maximum likelihood solution [Lorenc, 1986, Gebbie, 2004]. The weights are therefore chosen to be the inverse of the estimated noise or error in the observation (i.e. weight $=1 / \sigma^{2}$ ). This error estimate should account not only for the measurement error, but should include the expected error in representing the observation with the model.

Control variable penalties are given by

$$
J_{c t r l}=\sum(\text { control }- \text { prior })^{T} \mathbf{W}_{\text {ctrl }}(\text { control }- \text { prior })
$$

where the sum is taken over all control variables, prior is a vector of prior estimates for all control variables, control is a vector of the adjusted control variables, and $\mathbf{W}_{\text {ctrl }}$ is the control variable weighting matrix.

In structure this cost function is typical and could be solved in theory using standard least squares minimization techniques including direct matrix inversion. In practice, the problem is too large to solve in this fashion given current computational resources. Lagrange multipliers are used to recast the problem such that efficient solution techniques are possible.

Overview of Lagrange multipliers methodology. Excellent reviews of the application of Lagrange multipliers to ocean state estimation are available [Gebbie, 2004, Wunsch, 2006, Heimbach and Bugnion, 2009]. The terminology of Lagrange multipliers is chosen for its explicit reference to the mathematical approach. However, other terminologies are also popular: the adjoint method [Thacker and Long, 1988, Heimbach et al., 2005], the Pontryagin Principle [Wunsch, 2006], and 4D-Var [Courtier, 1997]. The terminology 4DVar is popular in atmospheric modeling contexts, where the adjoint method is applied to 
short estimation periods (on the order of hours). The term data assimilation is avoided here as it is often associated with sequential filtering approaches such as the Kalman filter [Kalman, 1960] that are more appropriate to forecasting than to climate studies. The methodology used here ensures that the model trajectory is free-running at all times; due to this key characteristic, it is possible to sensibly compute climate-relevant properties such as heat and mass budgets. A detailed discussion of the differences between data assimilation schemes such as the Kalman filter and state estimation approaches such as that used here is given in Wunsch and Heimbach [2012, in press].

The cost function $J$ of the previous section defines a constrained least squares problem. An application of the method of Lagrange multipliers transforms the problem into an unconstrained problem with an extended cost function that includes the model equations. To provide an illustrative but simplified discussion, the following discussion is limited to initial condition control variables $\mathbf{u}=\mathbf{x}\left(t_{0}=0\right)$. Note that there is no conceptual difference between control variables for initial conditions versus those for surface forcing or open boundary conditions - there is simply more bookkeeping for other control variables. The development given here follows that in Heimbach and Bugnion [2009]; interested readers can find the full analysis in Wunsch [2006]. Consider a time-dependent model integrated from $t_{0}=0$ to $t=t_{f}$ :

$$
\mathbf{x}(t)-L[\mathbf{x}(t-1)]=0
$$

where $\mathbf{x}(t)$ is the model state at time $t$ and $L$ is the model operator that steps the model forward in time. The derivatives of the cost function with respect to the control variables are required to estimate improved control variables (see next section for the algorithm). Renaming the traditional cost function (such as that of Equation 3.1) as $J_{0}$, an extended cost function is defined that appends the model equations:

$$
J=J_{0}[\mathbf{x}]-\sum_{t=1}^{t_{f}} \boldsymbol{\mu}^{T}(t)\{\mathbf{x}(t)-L[\mathbf{x}(t-1)]\}
$$

where $\boldsymbol{\mu}(t)$ are the Lagrange multipliers for each model state variable $\mathbf{x}(t)$. If the model 
Equation 3.2 holds, the numerical value of $J$ is equal to $J_{0}$ because the second term is identically zero. However, in the extended cost form, the model state, control and Lagrange multipliers can be treated as independent variables. The minimum of the extended cost function is found by requiring the partial derivatives of the cost function with respect to each variable to vanish independently for $t>0$.

$$
\begin{array}{rlrl}
\frac{\partial J}{\partial \boldsymbol{\mu}(t)} & =\mathbf{x}(t)-L[\mathbf{x}(t-1)]=0 & 1 \leq t \leq t_{f} \\
\frac{\partial J}{\partial \mathbf{x}(t)}=\frac{\partial J_{0}}{\partial \mathbf{x}(t)}-\boldsymbol{\mu}(t)+\left[\frac{\partial L[\mathbf{x}(t)]}{\partial \mathbf{x}(t)}\right]^{T} \boldsymbol{\mu}(t+1)=0 & 0<t<t_{f} \\
\frac{\partial J}{\partial \mathbf{x}\left(t_{f}\right)}=\frac{\partial J_{0}}{\partial \mathbf{x}\left(t_{f}\right)}-\boldsymbol{\mu}\left(t_{f}\right)=0 & t=t_{f} \\
\frac{\partial J}{\partial \mathbf{x}(0)}=\frac{\partial J_{0}}{\partial \mathbf{x}(0)}-\left[\frac{\partial L[\mathbf{x}(0)]}{\partial \mathbf{x}(0)}\right]^{T} \boldsymbol{\mu}(1) & t=t_{0}=0
\end{array}
$$

The model equations are recovered in Equation 3.3a. To find the Lagrange multipliers $\boldsymbol{\mu}(t)$, the final state at time $t_{f}$ (Equation 3.3c) is used to initialize a recursive, backwards in time computation using Equation 3.3b. The full gradient of the cost function with respect to the controls is obtained at time $t_{0}=0$ via Equation 3.3d. Thus the Lagrange multiplier accumulates the partial derivatives $\partial J_{0} / \partial \mathbf{x}$ from all time steps. These equations are solved iteratively.

In practice, the unavailability of the adjoint model, given by

$$
\left[\frac{\partial L[\mathbf{x}(t)]}{\partial \mathbf{x}(t)}\right]^{T}
$$

in the preceding equations, is the primary roadblock to the application of the method of Lagrange multipliers to nonlinear optimization problems using a complex numerical model such as the MIT GCM. The development of a hand-coded adjoint model is as difficult as the development of the original forward model. Such an effort is intractable for a large numerical code such as the MIT GCM, especially when the code is constantly being modified as is the case here. Fortunately, source code to source code translation tools have been devel- 
oped to automatically create the model adjoint through automatic differentiation [Giering and Kaminski, 1998]. A commercial tool called "Transformations of Algorithms in Fortran" (TAF) [Giering et al., 2005] is employed here. Since its origins, the MIT GCM has been developed with careful consideration to compatibility with the TAF tool, and more specifically with its non-commercial predecessor TAMC. The MIT GCM adjoint model has been successfully applied to estimate the state of the modern ocean in both global (e.g. Stammer et al. [2002], Wunsch and Heimbach [2009]) and regional (e.g. Gebbie et al. [2006], Mazloff et al. [2010], Fenty and Heimbach [2012]) configurations.

There are several components of the MIT GCM forward model code that are strongly nonlinear and which can cause severe instabilities in the adjoint model code. As has often been done in the above mentioned applications of the adjoint of the MIT GCM, we exclude from the adjoint the sea ice model, the GM/Redi eddy parameterization, and the GGL parameterization of vertical mixing. With this exclusion, the forward model state remains exact, but the adjoint model (i.e. the sensitivities of the cost function to the control parameters) is approximate.

A number of modest extensions to the MIT GCM have been made in this thesis. There have been no problems generating an appropriate model adjoint using TAF and there is every reason to believe that the generated adjoint is appropriate to our purposes.

A recipe for nonlinear optimization using the model adjoint. A broad approach has been outlined: to improve the fit of a model to data by computing improved initial and boundary conditions using the model adjoint. The following recipe details how this is done in practice and is based on the description given by Fenty [2010]. An iterative procedure is used to proceed from a first guess model solution to an improved estimate.

1. Obtain an initial estimate of ocean state by running the forward model with first guess initial and boundary conditions. Compute the cost function. This model state is called iteration 0 .

2. Use the cost function value to initialize the backwards integration of the model adjoint. Accumulate the Lagrange multipliers back to time $t_{0}=0$, at which point the 
sensitivity of the cost function to all control variables is known.

3. Use the sensitivity information in a downhill search to compute improved control variable estimates. The quasi-Newton, variable-storage algorithm of Gilbert and LeMarechal [1989] is used for this thesis, as in all of the ECCO solutions. Key aspects of this step include choosing the best downhill direction and deciding how large a change to make to the controls in any given iteration (small changes can result in unnecessary iterations; large changes can result in overshoots that can be difficult to recover from). As is the case with all non-linear optimization techniques, there is also the potential to become stuck in a local minimum. Ad-hoc approaches are used to recover from local minima.

4. Re-run the forward model using the improved estimate of initial and boundary conditions and compute the new cost function value. Generally the new solution is an improvement and the cost function will be smaller (the exception is that escaping from a local minimum in the cost function can require a few iterations in which the model-data fit worsens).

5. Repeat from step 2. Stop when the model-data misfit is acceptably small. Evaluate the posterior misfit statistics to check whether the solution satisfies the prior data and control variable uncertainty assumptions.

\subsubsection{Data constraints and uncertainties}

The model-data misfit component of the cost function, $J_{d a t a}$, is unique to each state estimate presented in this thesis. The form of $J_{d a t a}$ for the conceptually simplest state estimate - that 
of Section 3.3 - is useful as an an illustrative example:

$$
\begin{aligned}
J_{d a t a} & =\frac{2}{n y} \sum_{y=n y / 2}^{n y} \sum_{m=1}^{12}\left(\overline{\mathbf{T}}(y, m)-\mathbf{T}_{\mathbf{o c c a}}(m)\right)^{T} \mathbf{W}_{\mathbf{T}}\left(\overline{\mathbf{T}}(y, m)-\mathbf{T}_{\mathbf{o c c a}}(m)\right) \\
& +\frac{2}{n y} \sum_{y=n y / 2}^{n y} \sum_{m=1}^{12}\left(\overline{\mathbf{S}}(y, m)-\mathbf{S}_{\text {occa }}(m)\right)^{T} \mathbf{W}_{\mathbf{S}}\left(\overline{\mathbf{S}}(y, m)-\mathbf{S}_{\text {occa }}(m)\right)
\end{aligned}
$$

where the sums are taken over the second half of the state estimation period and the targets, $\mathbf{T}_{\text {occa }}(m)$ and $\mathbf{S}_{\mathbf{o c c a}}(m)$, are the OCCA estimates of monthly mean ocean temperatures and salinities (T/S) for month $m$ [Forget, 2010]. $\mathbf{W}_{\mathbf{T}}$ and $\mathbf{W}_{\mathbf{S}}$ are the weighting matrices on the $\mathrm{T} / \mathrm{S}$ misfits, given by the squared inverse of the prior uncertainty; prior uncertainty is given by the T/S uncertainties defined for modern hydrography by Forget and Wunsch [2007]. As noise covariances are poorly constrained, even for the modern ocean, data noise is assumed to be independent (off-diagonal elements in the weighting matrix are 0); this assumption is made throughout the thesis. $\overline{\mathbf{T}}(y, m)$ and $\overline{\mathbf{S}}(y, m)$ are the mean model temperature and salinity in month $m$ of simulation year $y$ and $n y$ is the number of years simulated in the model. Unstable model behavior can result from requiring the model to too tightly fit data directly adjacent to the northern and southern boundaries. To stabilize the solution, T/S uncertainties are linearly increased inside the 5-degree bands adjacent to the northern and southern boundaries (i.e. between $35^{\circ} \mathrm{S}-40^{\circ} \mathrm{S}$ and between $75^{\circ} \mathrm{N}$ and $80^{\circ} \mathrm{N}$ ).

This cost function form has been designed to permit a search for seasonal steady state solutions. As compared to typical ECCO cost functions, key differences include the following.

- Initial condition adjustments can introduce noise that is not of interest here. To focus on identifying a model state that is stable and close to the data at the end of the run, the cost is computed over the second half of the calculation only (i.e. $y=$ $n y / 2, n y / 2+1, \ldots n y)$.

- To search for a solution with an annual cycle that is stable in time, the model is asked to match the same seasonally varying target data set for every year included in the 
cost function.

- The factor $2 / n y$ is used to normalize the cost function by the number of years included; $J$ then represents an average per-year cost. In this formulation, the cost function shape does not depend directly on the run length, a property that proves useful as run length is extended (see Section 3.5).

\subsubsection{Control variables and uncertainties}

The choice of control variables is an important one: they should be quantities that have a significant impact on the solution and that are relatively uncertain. Here, the only control variables are model initial conditions, atmospheric forcing, and boundary conditions at $40^{\circ} \mathrm{S}$; although internal model parameters (such as the diapycnal diffusivity) could also be useful control variables, it is assumed here that internal model parameters are not as uncertain as atmospheric forcing and southern boundary conditions.

The model used here is non eddy-permitting, and is focused on large-scale ocean circulation. In these conditions, control adjustments that are spatially smooth and large-scale are preferred. Non-diagonal weighting matrices can be used to enforce a smoothness constraint on initial condition and atmospheric controls [Gebbie, 2004]. Recently, an alternative approach called the Smooth Package has been added by G. Forget to the MIT GCM. This implementation of Weaver and Courtier [2001] integrates a diffusion equation to smooth the control vector and gradients. A prescribed correlation length scale determines the diffusion coefficient. This new tool provides (1) an improved ability to enforce smoothness in control adjustments and (2) better conditioning of gradients, which assists the line search in improving the solution. In the configurations used here, this approach has typically reduced the number of iterations required to reach a satisfactory solution and produced atmospheric controls that are more spatially smooth. 
The basic form of $J_{c t r l}$ is as follows.

$$
\begin{aligned}
& J_{c t r l}= \\
& \left(\mathbf{T}_{\mathbf{0}}-\mathbf{T}_{\text {occaJan }}\right)^{T} \mathbf{W}_{\mathbf{T}}\left(\mathbf{T}_{\mathbf{0}}-\mathbf{T}_{\text {occaJan }}\right) \\
& +\left(\mathbf{S}_{\mathbf{0}}-\mathbf{S}_{\text {occaJan }}\right)^{T} \mathbf{W}_{\mathbf{S}}\left(\mathbf{S}_{\mathbf{0}}-\mathbf{S}_{\text {occaJan }}\right) \\
& +\sum_{v=1}^{6} \sum_{m=1}^{12}\left(\mathbf{A}(v, m)-\mathbf{A}_{\text {ncep }}(v, m)\right)^{T} \mathbf{W}_{\mathbf{A}}(v)\left(\mathbf{A}(v, m)-\mathbf{A}_{\text {ncep }}(v, m)\right) \\
& +\sum_{m=1}^{12}\left(\mathbf{T}_{\mathbf{O B}}(m)-\mathbf{T}_{\mathbf{O C C A}, 40^{\circ} \mathbf{S}}(m)\right)^{T} \mathbf{W}_{\mathbf{O B} \mathbf{T}}\left(\mathbf{T}_{\mathbf{O B}}(m)-\mathbf{T}_{\mathbf{O C C A}, 40^{\circ} \mathbf{S}}(m)\right) \\
& +\sum_{m=1}^{12}\left(\mathbf{S}_{\mathbf{O B}}(m)-\mathbf{S}_{\mathbf{O C C A}, 40^{\circ} \mathbf{S}}(m)\right)^{T} \mathbf{W}_{\mathbf{O B} \mathbf{S}}\left(\mathbf{S}_{\mathbf{O B}}(m)-\mathbf{S}_{\mathbf{O C C A}, 40^{\circ} \mathbf{S}}(m)\right) \\
& +\sum_{m=1}^{12}\left(\mathbf{U}_{\mathbf{O B}}(m)-\mathbf{U}_{\mathbf{O C C A}, 40^{\circ} \mathbf{S}}(m)\right)^{T} \mathbf{W}_{\mathbf{O B} \mathbf{U}}\left(\mathbf{U}_{\mathbf{O B}}(m)-\mathbf{U}_{\mathbf{O C C A}, 40^{\circ} \mathbf{S}}(m)\right) \\
& +\sum_{m=1}^{12}\left(\mathbf{V}_{\mathbf{O B}}(m)-\mathbf{V}_{\mathbf{O C C A}, 40^{\circ} \mathbf{S}}(m)\right)^{T} \mathbf{W}_{\mathbf{O B} \mathbf{V}}\left(\mathbf{V}_{\mathbf{O B}}(m)-\mathbf{V}_{\mathbf{O C C A}, 40^{\circ} \mathbf{S}}(m)\right)
\end{aligned}
$$

Equations $3.5 \mathrm{a}$ and $3.5 \mathrm{~b}$ penalize departures of the adjusted model starting conditions in January from the OCCA fields for January; uncertainties are given by Forget and Wunsch [2007] (the same uncertainties that were used in the data cost function in Equation 3.4).

Equation $3.5 \mathrm{c}$ describes the cost function penalty terms for atmospheric adjustments where $v$ indexes the six atmospheric variables used as controls: shortwave radiation, 2-m air temperature, 10-m zonal wind speed, 10-m meridional wind speed, 2-m specific humidity, and precipitation. $\mathbf{A}(v, m)$ is the adjusted estimate for atmospheric forcing variable $v$ in month $m$ and $\mathbf{A}_{\text {ncep }}(v, m)$ is the prior estimate for the same variable. The adjusted atmospheric forcing is a repeating annual cycle with monthly fields. Since atmospheric adjustments are likely to be sensitive to assumed uncertainties, the choice of these uncertainties is important. Modern atmospheric forcing is more poorly known than might be expected [Bromwich et al., 2007]. Prior uncertainties on atmospheric adjustments are chosen to be spatially uniform with values defined in Table 3.2. $\mathbf{W}_{\mathbf{A}}(v)$, the weighting matrix 
for atmospheric variable $v$, is given by the squared inverse of the relevant uncertainty from Table 3.2. As was the case with data constraints, masking is employed in 5-degree bands adjacent to the northern and southern boundaries. Within these bands, atmospheric control uncertainties are linearly reduced as the boundary is approached; this approach serves to stabilize the model by reducing unrealistically large atmospheric adjustments that otherwise occur near the boundary.

\begin{tabular}{|l|l|}
\hline Shortwave radiation & $10 \mathrm{~W} / \mathrm{m}^{2}$ \\
2-m air temperature & $1{ }^{\circ} \mathrm{C}$ \\
10-m zonal wind speed & $1 \mathrm{~m} / \mathrm{s}$ \\
10-m meridional wind speed & $1 \mathrm{~m} / \mathrm{s}$ \\
2-m Specific humidity & $10^{-3} \mathrm{~kg} / \mathrm{kg}$ \\
Precipitation & $210^{-8} \mathrm{~m} / \mathrm{s}$ \\
\hline
\end{tabular}

Table 3.2: Prior uncertainties for atmospheric controls in modern state estimates.

In a regional model, and particularly over long simulation times, appropriate estimates of open boundary conditions are key. As these conditions are uncertain, control variables are defined for the southern boundary conditions of monthly mean temperature, salinity, and zonal/meridional velocities. Equations $3.5 \mathrm{~d}, \mathrm{e}, \mathrm{f}$, and $\mathrm{g}$ define penalties on the T/S/U/V adjustments where $\mathbf{T} / \mathbf{S} / \mathbf{U} / \mathbf{V}_{\mathbf{O B}}$ are the adjusted properties, $\mathbf{T} / \mathbf{S} / \mathbf{U} / \mathbf{V}_{\mathbf{O C C A}, \mathbf{4 0}}{ }^{\circ}$ are the first guess estimates, and $\mathbf{W}_{\mathbf{O B} \text { T }} / \mathbf{W}_{\text {OB S }} / \mathbf{W}_{\text {OB U }} / \mathbf{W}_{\text {OB V }}$ are the weighting matrices. Uncertainties in $40^{\circ} \mathrm{S}$ temperature and salinity are assumed to be uniform in longitude but varying in depth; the mean of Forget and Wunsch [2007] computed for each model layer is used. Computing appropriate adjustments to meridional and zonal velocities has historically been a major technical challenge (see Gebbie [2004] for a thorough review). A particular difficulty has been that the ocean state is highly sensitive to barotropic flow at open boundaries and even small adjustments to the barotropic component lead to large and often physically unreasonable net volume fluxes into or out of the domain. An addition to the MIT GCM was made recently by M. Mazloff that permits an improved conditioning on this problem by decomposing the control problem into barotropic and baroclinic modes, with the mode 
amplitude as the control variable. To use this new capability, a modal decomposition was generated using the mean OCCA stratification at $40^{\circ} \mathrm{S}$. Mode uncertainties impact how much energy is placed in different vertical wavenumbers. Acceptable adjustments were found with relative (nondimensional) uncertainties of 1 for the first 15 baroclinic modes, 0.5 on the last 35 baroclinic modes, and 0.05 on the barotropic mode. Consideration of interannual changes in mass storage in the Atlantic Ocean are not permitted by our seasonal steady state formulation. A low relative uncertainty is used for the barotropic mode so that meridional velocity adjustments have nearly balanced net transport at each longitude of the boundary. Any remaining net flux across the boundary is compensated for using a small barotropic correction term applied uniformly at all grid points of the boundary.

\subsubsection{A focus on equilibrium ocean estimation}

Chapter 1 introduced the potential scientific benefit of equilibrium ocean estimation (EOE): the development of estimates of ocean state that are (1) within error bounds of a given dataset, (2) consistent with known oceanic dynamics, and (3) consistent with a seasonal steady state assumption. The following features of the state estimation approach described in this section have been specifically designed to facilitate EOE.

- The cost function is applied over only the second half of the years simulated in the state estimate. This de-emphasizes the direct effects of initial condition adjustments and favors simulations with minimal drift.

- For simulation years included in the cost function, the model is required to fit the same data in every year, a framing that favors low-drift solutions with low interannual variability.

- Atmospheric forcing and southern boundary conditions are applied in a repeating seasonal cycle, both for first guess values and for control adjustments.

- Significant effort was made to stabilize the adjoint model so that meaningful solution improvement could be obtained, even in longer state estimates. As an example, the 
graduated masking of uncertainties at the northern and southern boundaries improved the utility of the adjoint model in longer runs.

\subsection{State estimation with modern T/S information: the Modern_TS estimate}

In this section, we evaluate whether our regional model can adequately reproduce the properties of the OCCA state estimate in the second year of a two-year state estimate. Success is not a given as differences between the model configurations are many: OCCA is a global model while ours is regional, the OCCA atlas is an average of three one-year state estimates whereas our approach considers the fit of the second year of a two-year estimate, OCCA works to fit observational records whereas we work to fit the OCCA atlas properties, OCCA uses 6-hourly atmospheric forcing while we use daily mean, and OCCA permits 10-day control adjustments while we permit monthly mean.

\subsubsection{Cost reduction}

This section is focused on the reduction of cost via the optimization procedure; spatial distributions of misfits and controls will be described in Section 3.3.2. Figure 3-1 (upper plot) shows that the iterative optimization procedure reduces the total cost by over $50 \%$ in 20 iterations; control variable costs (see expanded view in the lower plot of Figure 3-1) start at zero in iteration zero (an unadjusted iteration with zero control variable adjustments) and then increase as larger control variable adjustments are used. Although control adjustments increase in size with iteration number, their cost contribution remains a minor fraction of the total - the control costs are barely visible as compared to data misfit costs in the upper plot of Figure 3-1.

Several features of Figure 3-1 are typical of the many optimizations performed for this thesis: early iterations employ small control adjustments and achieve relatively small total 


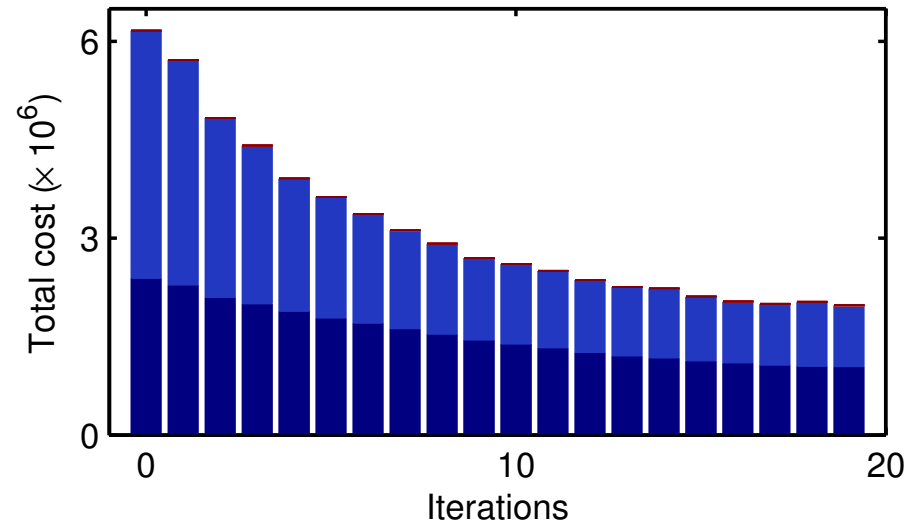

\begin{tabular}{|l}
\hline S. boundary controls \\
\hline Shortwave radiation \\
\hline Precipitation \\
Specific humidity \\
Air temperature \\
V wind speed \\
U wind speed \\
Initial salinity \\
Initial temperature \\
Misfit to salinity \\
Misfit to temperature
\end{tabular}

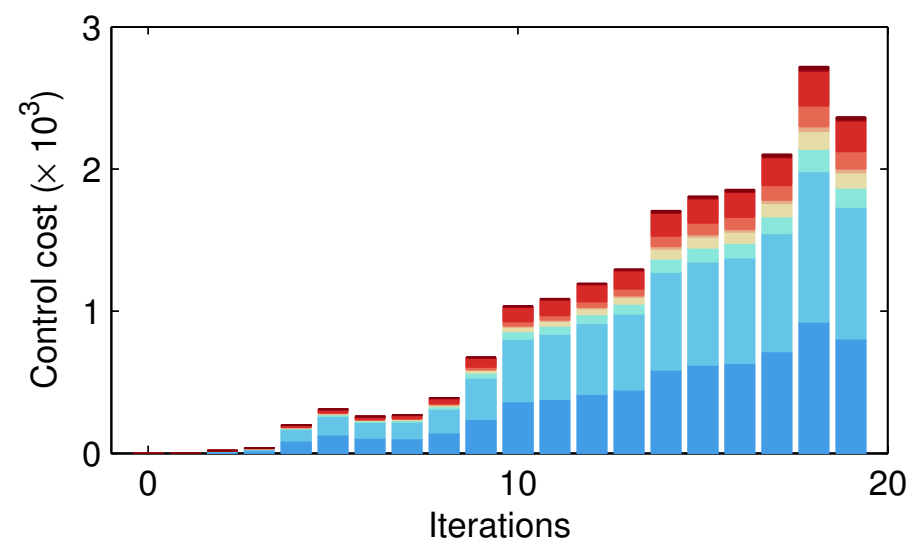

20

Figure 3-1: Analysis of cost components with iteration number for year two of a two-year state estimate constrained by OCCA temperatures and salinities. A significant reduction in total cost (upper plot) is achieved from the unadjusted iteration 0 to the final iteration 19; the cost components for misfit to OCCA temperature and salinity (the two darkest blue bars) dominate the total cost so that control costs (all other colors) are nearly invisible. The lower plot shows just the evolution of control costs on an expanded vertical axis.

cost reductions, increasingly large adjustments in later iterations achieve lower and lower total costs, and eventually any additional increases in the magnitude of the control variables result in increases in overall cost, rather than decreases (due to the penalty terms on control variable adjustments). For the configurations used in this thesis, our experience has been that continuing the optimization beyond the leveling off phase seen in the later iterations of Figure 3-1 has generally provided only very minimal additional reductions in cost. On the other hand, temporarily changing the relative weighting of different terms (thereby 
changing the cost function shape) did prove a useful strategy in achieving additional cost reductions. Note that such changes in the shape of the cost function are temporary only; all costs reported in the thesis are computed using unmodified cost functions (that is, using the cost functions described in the thesis).

The strategy of changing cost function shape was employed to further reduce the costs beyond those achieved in iteration 19 of Figure 3-1. After an additional 15 iterations, total costs were reduced by an additional 15\% beyond those of iteration 19. The final improved estimate will hereafter be referred to as state estimate "Modern_TS".

Before proceeding it should be noted that in general in this thesis we do not go to extraordinary lengths to find the absolute lowest possible cost; other minima with lower cost values likely exist and could potentially be found through additional effort (more iterations or additional temporary changes in cost function shape). Our goal here is to obtain an estimate of ocean state that is within uncertainty of the data, beyond which point, different solutions are statistically indistinguishable (i.e. the data are insufficient to differentiate between the solutions).

\subsubsection{Consistency of the state estimate}

The consistency of the Modern_TS solution will now be evaluated; all analyses apply to year two of this estimate. The empirical cumulative distribution function (CDF) of signed, normalized misfits (model-target $/ \sigma$ ) is used as a diagnostic for the fit of the state estimate to the target dataset (e.g. OCCA in this case). The CDF is a plot of $F(x)$ against $x$ where

$$
F_{\mathrm{X}}(x)=\frac{\text { number of occurrences for which } \mathrm{X} \leq x}{\text { total number of occurrences }} .
$$

Under the assumption that the normalized misfits are normally distributed, a solution can be accepted at the $95 \%$ confidence level if $95 \%$ or more of the signed, normalized misfits are inside the range of $[-2,+2]$ (i.e. $95 \%$ or more of the model values are within $2 \sigma$ of the data). Figure 3-2 shows that, within rounding errors, 91\% (100\%) of modeled temperatures 

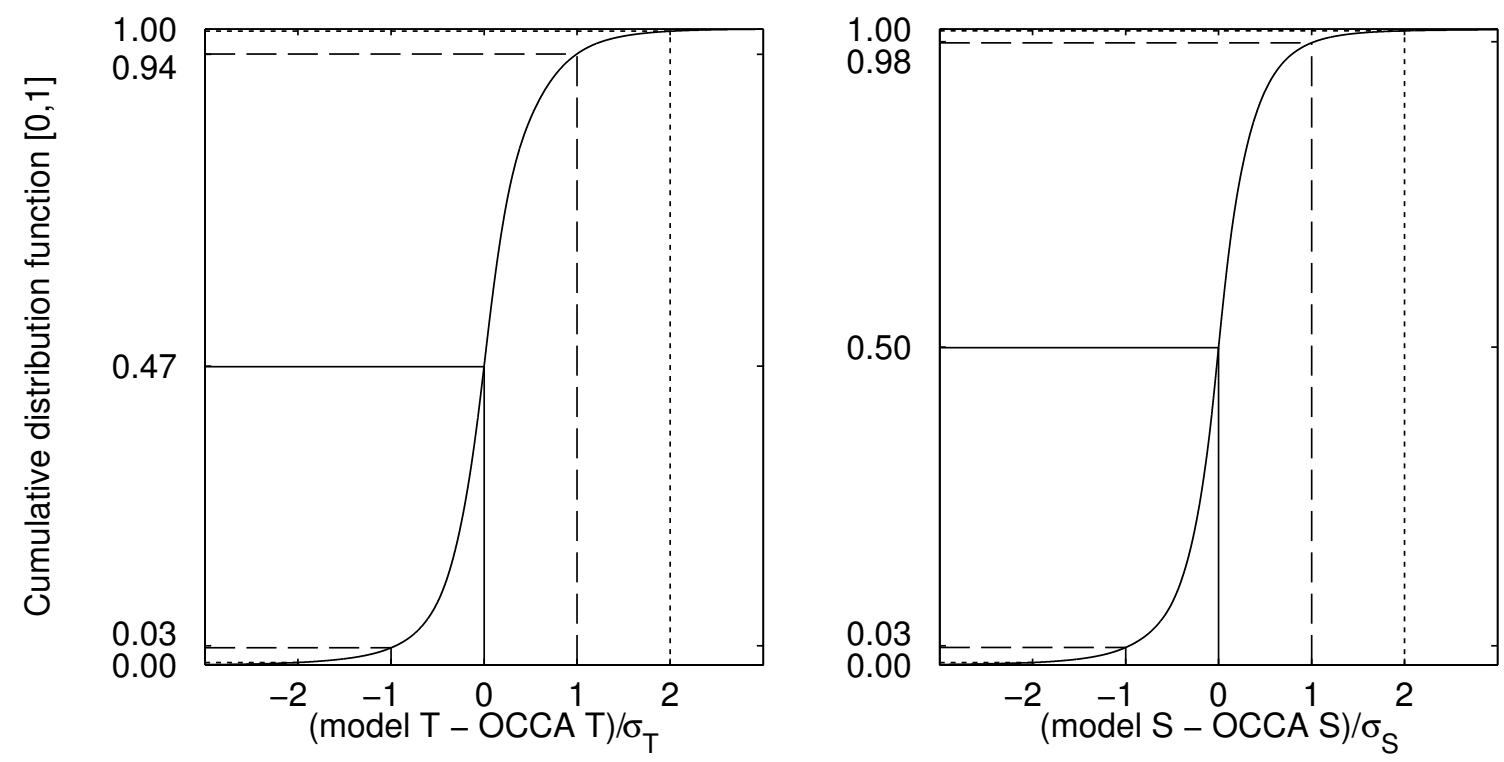

Figure 3-2: Cumulative distribution functions for signed, normalized misfits of Modern_TS temperatures (left) and salinities (right) to OCCA. The full y-scale is [0,1] in all CDF plots of this thesis; y-axis labels specify the fraction of values below \pm 2 (dotted lines), below \pm 1 (dashed lines), and below 0 (solid line). The fraction below zero indicates whether Modern_TS is biased relative to OCCA, and the fraction inside the $\pm 1( \pm 2)$ lines indicates what fraction of Modern_TS values are within $1 \sigma(2 \sigma)$ of OCCA.

are within $1 \sigma(2 \sigma)$ of OCCA and $95 \%(100 \%)$ of modeled salinities are within $1 \sigma(2 \sigma)$ of OCCA. Also, 47\% (50\%) of the modeled temperatures and salinities are lower than those of OCCA, indicating there is no serious bias in the Modern_TS T/S relative to OCCA.

Control variable adjustments should also be checked a posteriori to ensure consistency with the prior uncertainty assumptions. Figure 3-3 shows CDFs of the normalized control adjustments ( $\left.\frac{\text { adjusted control - prior control }}{\sigma}\right)$ for all of the atmospheric control variables; prior uncertainties $\sigma$ were defined in Table 3.2. Nearly 100\% of the adjusted control variables are within $\pm 2 \sigma$ of the prior (first guess) estimates. The horizontal lines in Figure 3-3 show what fraction of normalized control variable adjustments falls below zero. Specific humidity, air temperature, and shortwave radiation adjustments show particularly large biases; model adjustments more frequently cause reductions, rather than increases, in incoming solar radiation, air temperature, and specific humidity. Increases are about as frequent as 
decreases for precipitation and the wind speed variables.

Figure 3-4 shows a similar analysis for initial condition and southern boundary control variables. Initial condition uncertainties are given by the hydrographic uncertainties of Forget and Wunsch [2007]; southern boundary uncertainties were defined on page 100. As was the case for atmospheric controls, adjusted initial condition controls are nearly all within $\pm 2 \sigma$ of prior estimates, and appear relatively unbiased. Adjusted southern boundary controls are extremely small - note the x-axis in the right-hand plot of Figure 3-4 is highly expanded. Control adjustment size is related not only to the prior uncertainty, but also to the sensitivity of the model to the controls in question; we hypothesize that in a short simulation such as the current one, relatively few regions of the model are affected by the southern boundary conditions. In this case, modifying these variables does not significantly reduce the cost function and so is not warranted.

Based on the CDF diagnostics described above, the Modern_TS solution appears acceptable. However, it is important to examine the spatial distributions of properties and misfits to identify potential issues such as large scale biases. As shown in the top row of Figure 3-5, near sea surface temperatures and salinities (NSST and NSSS) in Modern_TS appear plausible. Misfits to OCCA (Figure 3-5, middle row) are small relative to the assumed prior uncertainty estimates of Forget and Wunsch [2007], as evidenced by the observation that normalized misfits (Figure 3-5, bottom row) are below one nearly everywhere (indicating that Modern_TS is within uncertainty of OCCA nearly everywhere). The primary exception is seen in the eastern equatorial region, where normalized NSST misfits are order two or higher. Model properties in this region may be particularly sensitive to the choice of internal model parameters, such that it is not possible to find adjustments in the initial and boundary condition control parameters that cause Modern_TS to reproduce OCCA properties here. This region is also one that may be difficult to simulate accurately in coupled models: the PMIP2 coupled models reviewed in Section 2.3.1 all showed temperatures in this region that were $2-4^{\circ} \mathrm{C}$ warmer than modern in simulations of the pre-industrial climate (see page 74). 

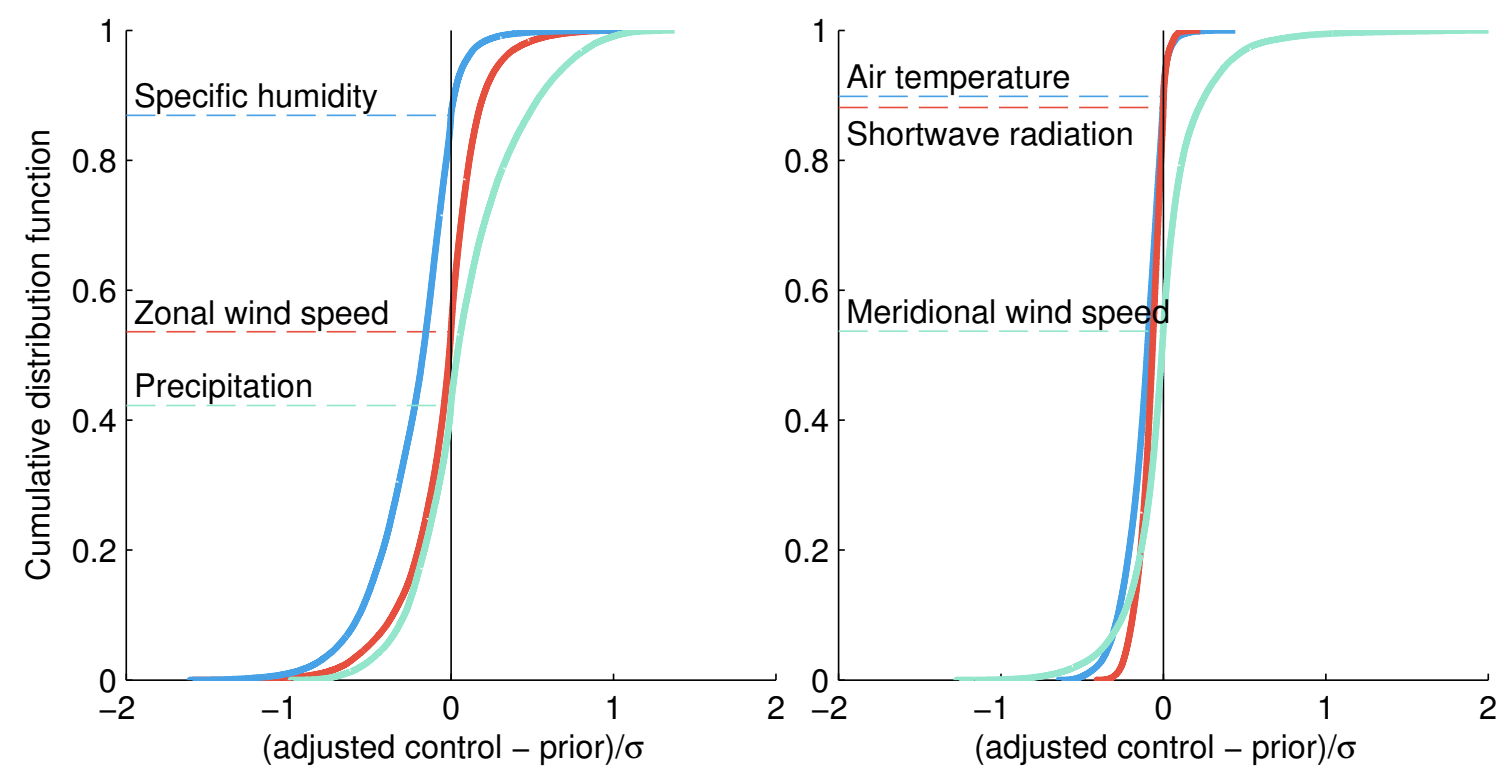

Figure 3-3: Cumulative distribution functions for Modern_TS adjustments to atmospheric control variables, normalized by prior uncertainty assumptions (final control - prior control) $/ \sigma$. Horizontal lines indicate what fraction of values fall below zero.
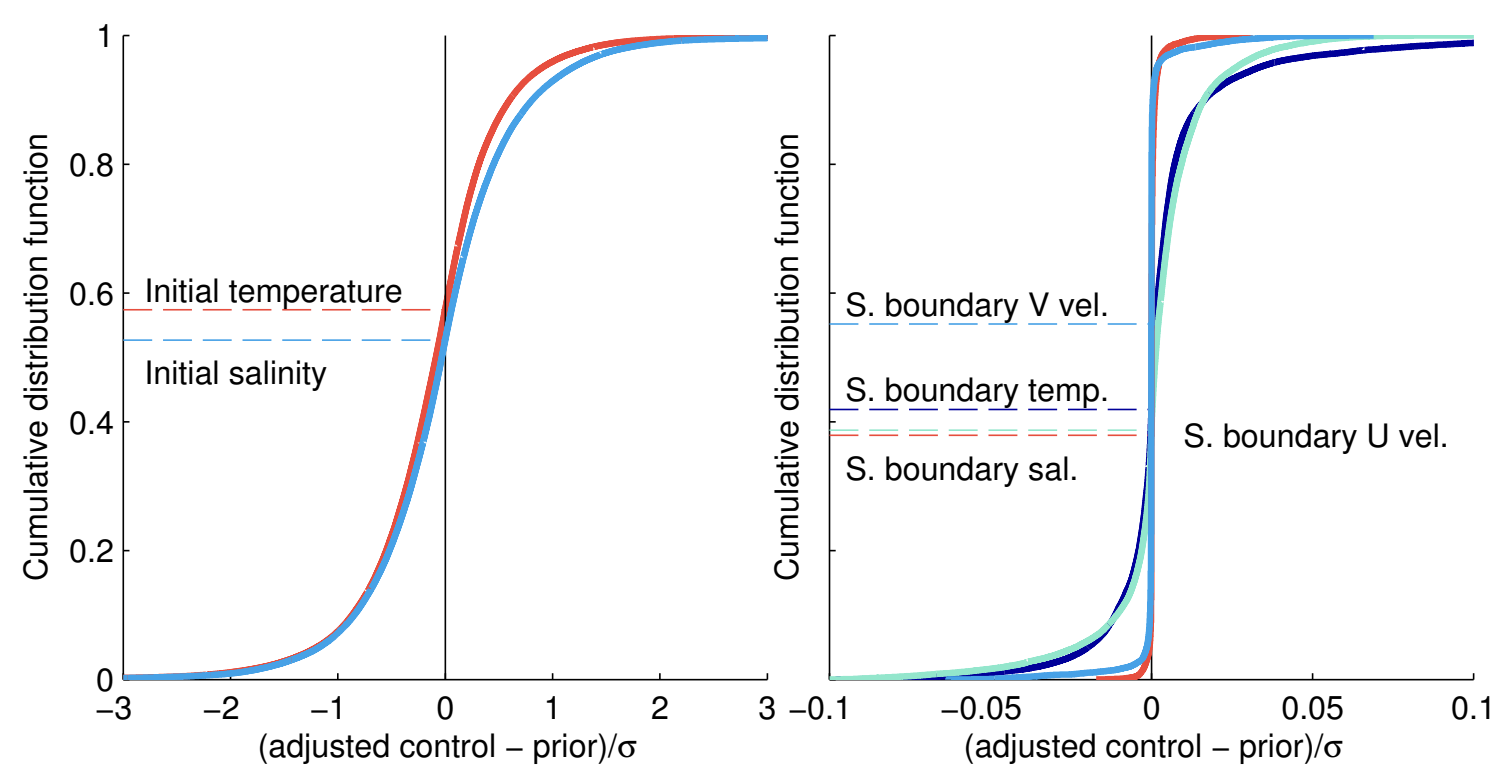

Figure 3-4: Cumulative distribution functions for Modern_TS adjustments to initial conditions (left) and to conditions at the southern boundary of the model (right); all are normalized by prior uncertainty assumptions (final control - prior control) $/ \sigma$. Southern boundary condition controls are very small relative to prior uncertainties (note the expanded $\mathrm{x}$-axis on the right). 


\section{Near sea surface conditions}
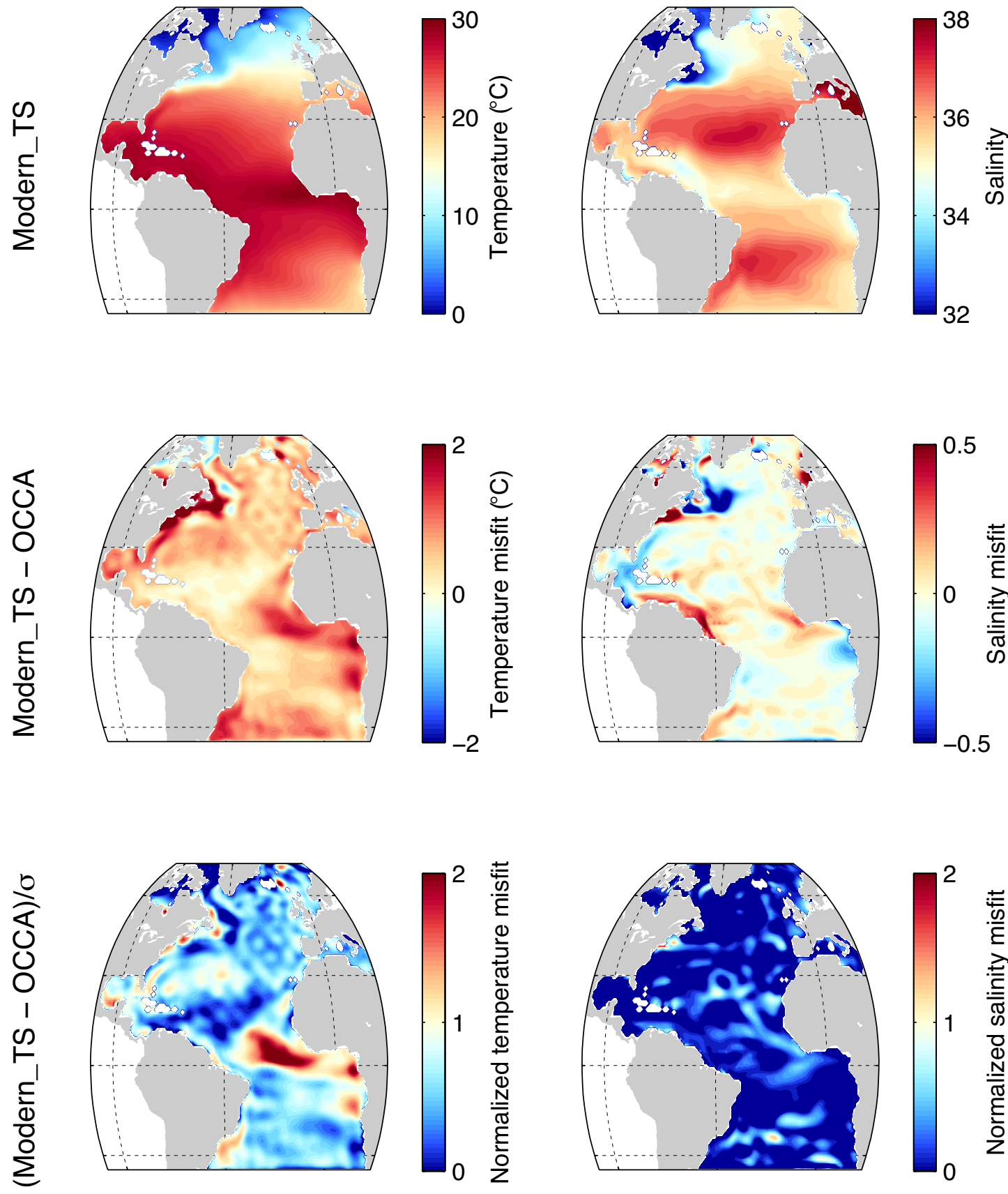

Figure 3-5: Modern_TS annual mean near sea surface temperature (top left) and salinity (top right), misfit to OCCA (middle row), and normalized misfits (bottom row). Normalized misfits (Modern_TS - OCCA)/ $\sigma$ of order one imply that Modern_TS is within uncertainty of OCCA. 
The Gulf Stream region also shows large misfits (middle row of Figure 3-5); the Gulf Stream path is a feature that is often very difficult to accurately capture in numerical models, and that may be quite sensitive to chosen parameter values [Chassignet and Marshall, 2008]; it is therefore not surprising that the models have different Gulf Stream paths. Note that the Gulf Stream is also a region of high variability so that the assumed prior uncertainties are large in this region, which explains why normalized misfits for this region are relatively small. A final observation is that temperature misfits are positive nearly everywhere (Figure 3-5, middle left), a clear indication that Modern_TS exhibits a warm bias in NSSTs relative to OCCA. The source of this bias is unknown, but should be kept in mind.

A similar analysis of Modern_TS and its misfits at a depth of $300 \mathrm{~m}$ is shown in Figure 3-6. Misfits at this depth are order $\pm 1{ }^{\circ} \mathrm{C}$ and 0.1 in salinity; normalized misfit maps show that these differences are small compared to the assumed prior uncertainties. Interesting patterns are evident in the misfit maps: the Gulf Stream region and southern edge of the model are both generally too cold and fresh in Modern_TS as compared to OCCA. Similar to the discussion for surface properties, the Gulf Stream path is highly dependent on the specifics of the model, so that an exact match between Modern_TS and OCCA may not be possible. The Modern_TS to OCCA differences near the southern boundary may be associated with difficulties in properly controlling the $40^{\circ} \mathrm{S}$ open boundary conditions.

At 4000 m (Figure 3-7), Modern_TS properties are fairly uniform and misfits are small (as are normalized misfits). In a two-year state estimate such as this one, properties at 4000 $\mathrm{m}$ are defined largely by initial conditions. Thus, the properties of OCCA are reproduced very closely via adjustments to initial condition controls.

That the normalized misfits are generally much less than one in Figures 3-5-3-7 is an indication that either the prior uncertainties were too large, or that the model is overfitting the target. Indeed, the uncertainties of Forget and Wunsch [2007] are not designed for the current exercise of using an existing ocean state estimate (OCCA) as a target for a new state estimate. These issues should be considered in future investigations; for our purposes here, we simply conclude that the Modern_TS estimate setup appears sufficient to reproduce 


\section{Properties at $300 \mathrm{~m}$}
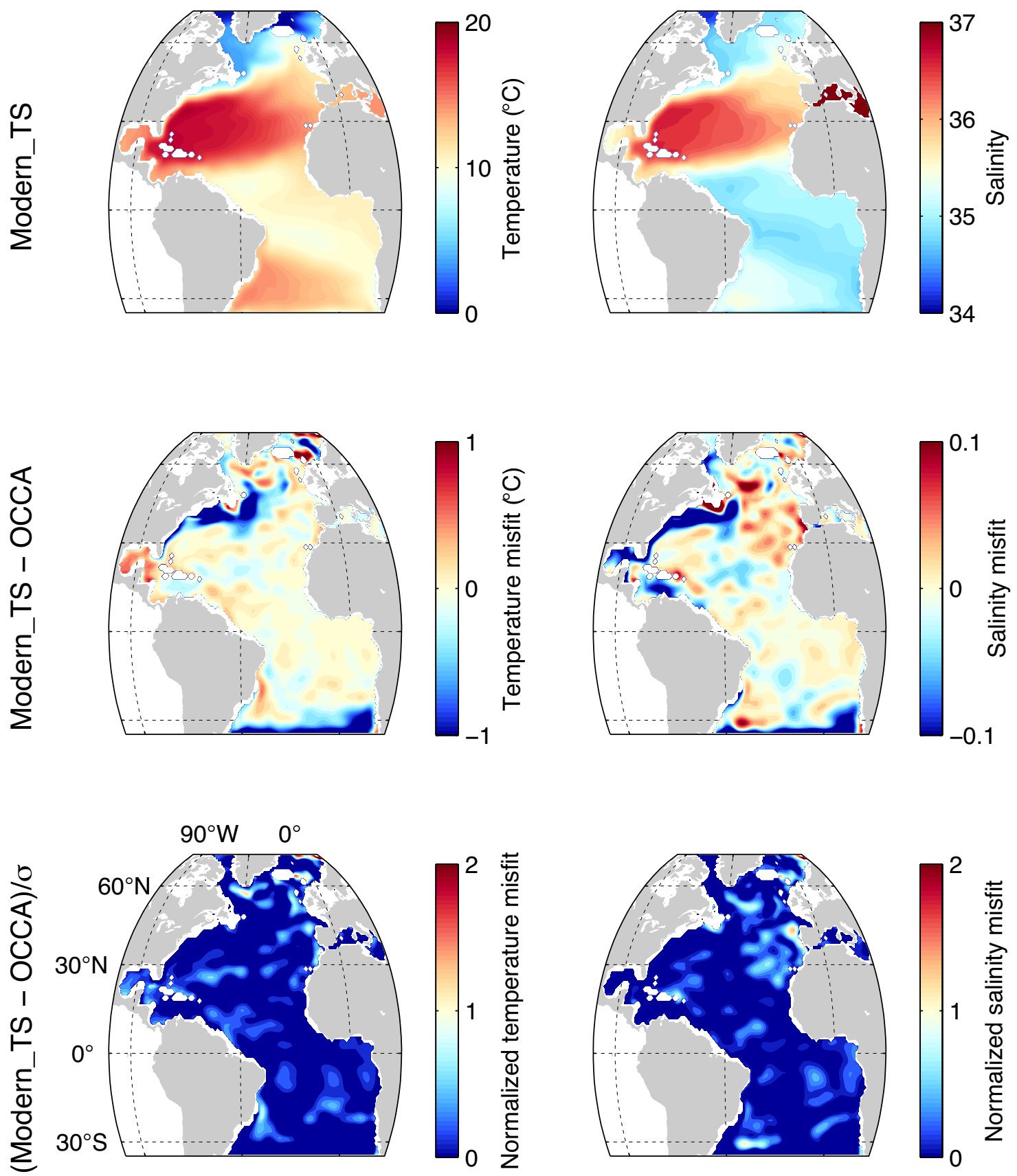

Figure 3-6: Modern_TS annual mean $300 \mathrm{~m}$ temperature (top left) and salinity (top right), misfit to OCCA (middle row), and normalized misfits (bottom row). 


\section{Properties at $4000 \mathrm{~m}$}
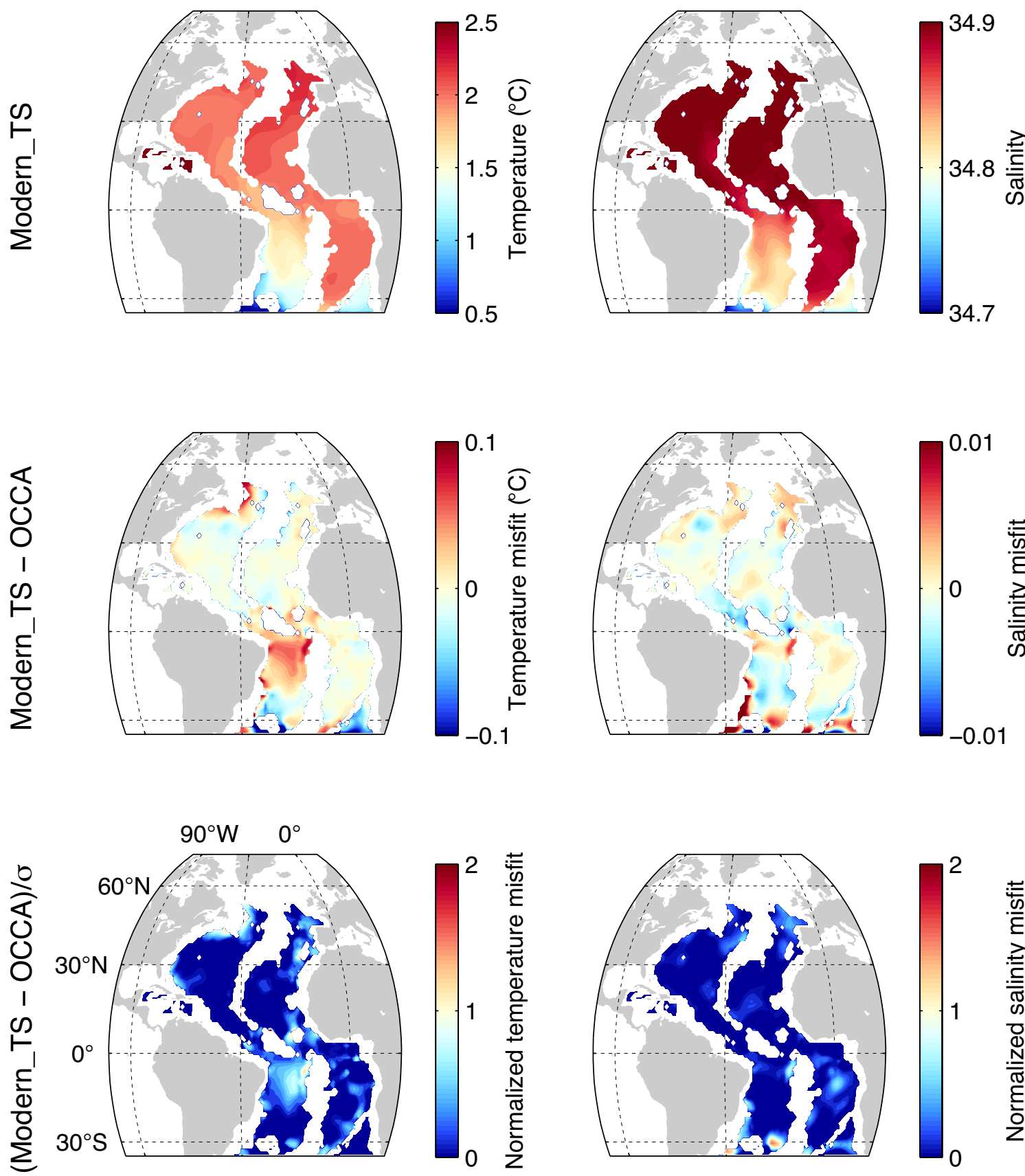

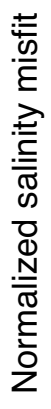

Figure 3-7: Modern_TS annual mean $4000 \mathrm{~m}$ temperature (top left) and salinity (top right), misfit to OCCA (middle row), and normalized misfits (bottom row). 
OCCA properties in a two year state estimate.

\subsubsection{Inferred properties}

As Modern_TS employs only T/S-based constraints, all other model properties are inferences based on these data, the first guess initial and boundary conditions, and the equations of motion. As an example, there is no transport-based constraint specifying that western boundary currents must exist, nor do we use altimetric constraints; the existence of all transports is inferred from the equations of motion and the $\mathrm{T} / \mathrm{S}$ constraints.

Flow patterns. Annual mean horizontal velocities for Modern_TS and OCCA, and the difference in magnitude, are shown in Figure 3-8 at depths of $55 \mathrm{~m}$ and $1160 \mathrm{~m}$. The dominant flow patterns in Modern_TS and OCCA are very similar. Misfits in current speeds are about $20 \%$ of the magnitude of the OCCA current speed at a depth of $55 \mathrm{~m}$. At a depth of $1160 \mathrm{~m}$, regions with relatively high current speeds (e.g. near $30^{\circ} \mathrm{N}$ ) show misfits that are also about $20 \%$ of OCCA speeds, while regions with lower current speeds in OCCA (e.g. near $30^{\circ} \mathrm{N}$ ) show misfits that are on the same order as the OCCA current speeds. Note that normalized misfits in temperature and salinity at a depth of $1160 \mathrm{~m}$ are within prior uncertainties almost everywhere (not shown). Since Modern_TS is constrained solely by OCCA temperature and salinity, it can be inferred that, given our prior uncertainty assumptions, the target temperatures and salinities are not sufficiently well known to resolve the smaller scale flow patterns at $1160 \mathrm{~m}$ in Modern_TS.

Mean dynamic topography (MDT) is the difference between the time averaged sea surface and an equipotential surface of Earth's gravity field; assuming the large-scale ocean circulation is close to geostrophic balance, MDT is a useful representation of the long term mean circulation. Figure 3-9 compares MDT in Modern_TS to that in the Earth Gravitational Model (EGM, http://earth-info.nga.mil/GandG/wgs84/gravitymod/egm2008/). To permit comparison of the two estimates, the MDT in Modern_TS has been shifted by a uniform offset of $0.17 \mathrm{~m}$ (this is the mean offset between the two estimates between $20^{\circ} \mathrm{S}$ and $20^{\circ} \mathrm{N}$ ). Overall, the Modern_TS MDT field appears somewhat flatter (less energetic) 


\section{$55 \mathrm{~m}$ depth}
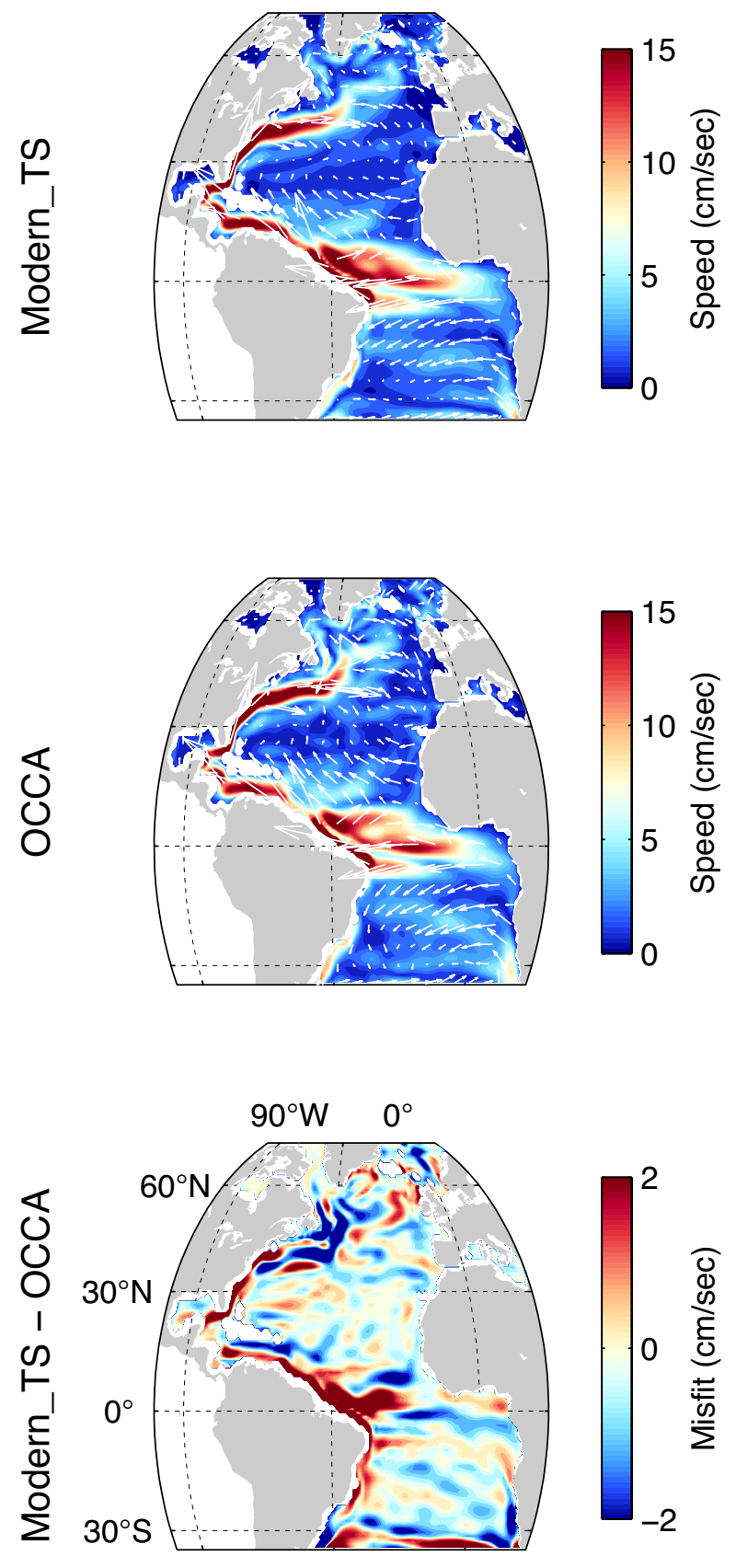

1160 m depth
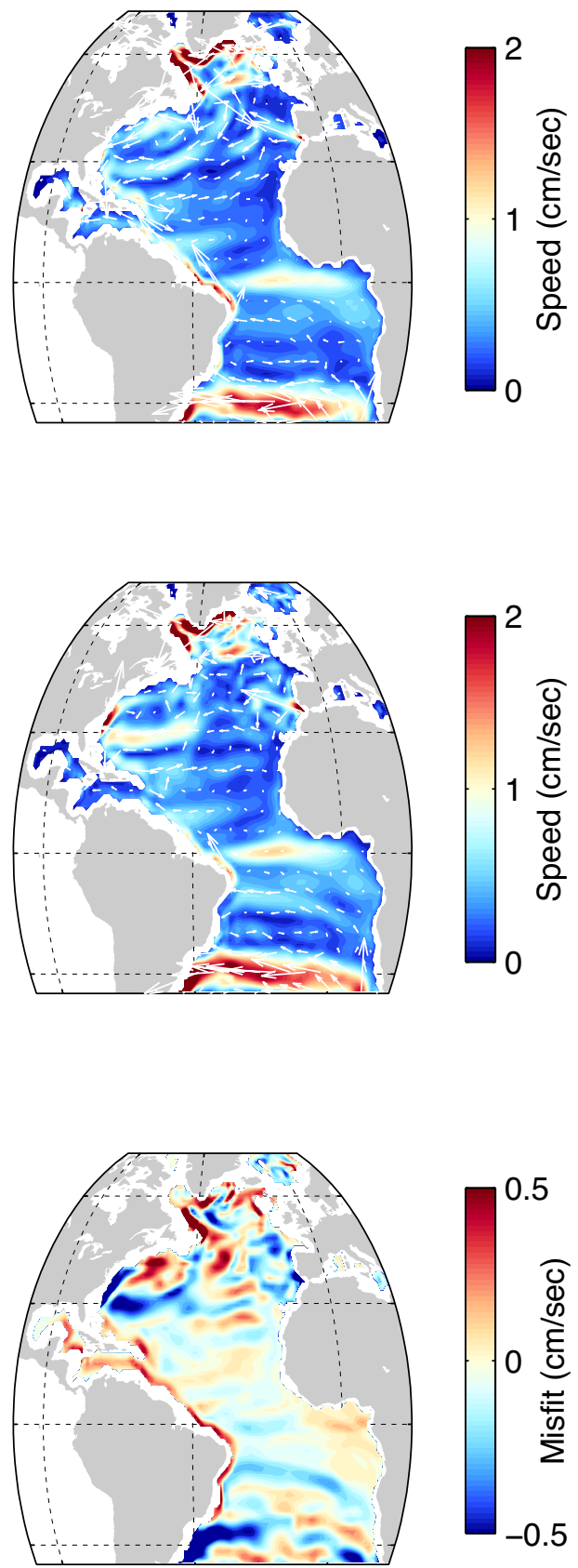

Figure 3-8: Annual mean current speed (contours) and direction (arrows) at $55 \mathrm{~m}$ (lefthand plots) and at $1160 \mathrm{~m}$ (right-hand plots). The plots include Modern_TS currents (top row), OCCA currents (middle row) and the difference in speed (Modern_TS - OCCA, lower plots). 
than the EGM estimate: Modern_TS MDT is less positive in the subtropical gyre and less negative in the subpolar gyre. The Gulf Stream path also appears to be shifted southward in Modern_TS relative to the EGM estimate. Significant differences are also apparent near the northern and southern boundaries, differences that are likely attributable to the artificial nature of these boundaries in the region model configuration used in Modern_TS.

Transports. As shown in Figure 3-10 (left side), the Modern_TS Atlantic meridional overturning circulation (AMOC) has the same maximum strength of $16 \mathrm{~Sv}$ (1 Sv $=10^{6}$ $\mathrm{m}^{3} / \mathrm{sec}$ ) as OCCA and the general shape of the two streamfunctions is similar. These AMOC transports agree with the estimate of $16 \pm 2 \mathrm{~Sv}$ found by Ganachaud [2003] and are close to the estimate of 18 Sv found by Talley et al. [2003].

In considering MOC transports, it is important to remember that the MOC is a highly integrated quantity and much important information about the circulation is missing. Indeed, additional information about the transports making up the MOC is required to gain an understanding of the underlying ocean circulation. Figure 3-10 (right side) shows a breakdown of transports at $29^{\circ} \mathrm{N}$ for OCCA and Modern_TS. Net transports are computed inside longitude-depth ranges as follows: Gulf Stream: $80^{\circ} \mathrm{W}$ to $73.4^{\circ} \mathrm{W}, 900 \mathrm{~m}$ to surface; Deep Western Boundary Current (DWBC): $77^{\circ} \mathrm{W}$ to $68.4^{\circ} \mathrm{W},-5700$ to $-900 \mathrm{~m}$; Upper Interior flow: $73.4^{\circ} \mathrm{W}$ to $12^{\circ} \mathrm{W},-900 \mathrm{~m}$ to surface; Middle Interior flow: $68.4^{\circ} \mathrm{W}$ to $12^{\circ} \mathrm{W},-2800$ to $-900 \mathrm{~m}$; and Deep Interior flow: $68.4^{\circ} \mathrm{W}$ to $12^{\circ} \mathrm{W},-5900$ to $-2800 \mathrm{~m}$. The Modern_TS Gulf Stream is 5\% stronger than that of OCCA, while the Modern_TS DWBC is 32\% stronger. Increased DWBC transport in Modern_TS is compensated by damped interior return flow so that the net deep return transport (the MOC) is similar in the two circulation estimates.

OCCA and Modern_TS inferred transports are now compared against independent estimates (these estimates are independent because neither OCCA nor Modern_TS is directly constrained by volume transport estimates). Baringer and Larsen [2001] compute net transport of the Florida Current at $27^{\circ} \mathrm{N}$ based on cable measurements between Florida and the Bahamas. They find a mean transport over 1982 - 1998 of 32 Sv. The estimated Gulf Stream transports shown in Figure 3-10 are computed slightly farther north at $28.5^{\circ} \mathrm{N}$, a 

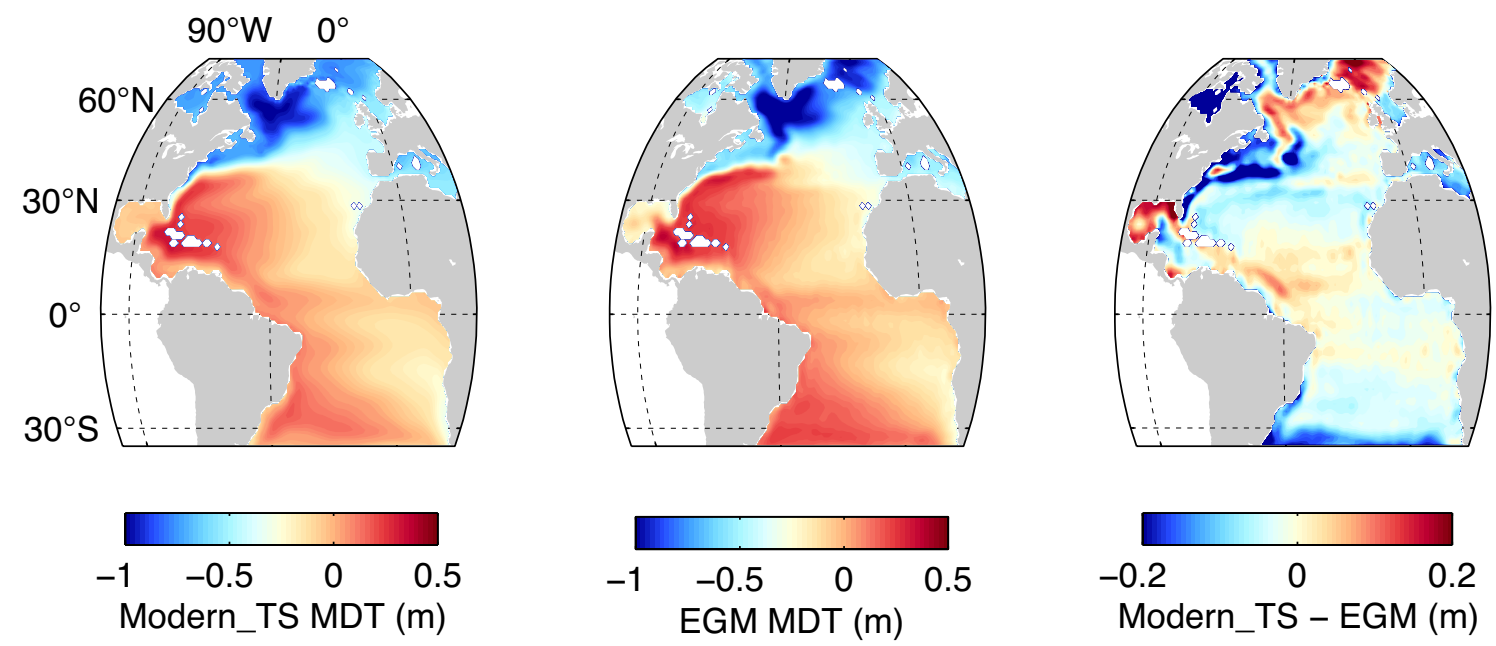

Figure 3-9: Mean dynamic topography (MDT) in meters for Modern_TS (left) and for the Earth Gravitational Model (EGM) estimate (center). Differences are shown on the right.

OCCA MOC and transports at $29^{\circ} \mathrm{N}$
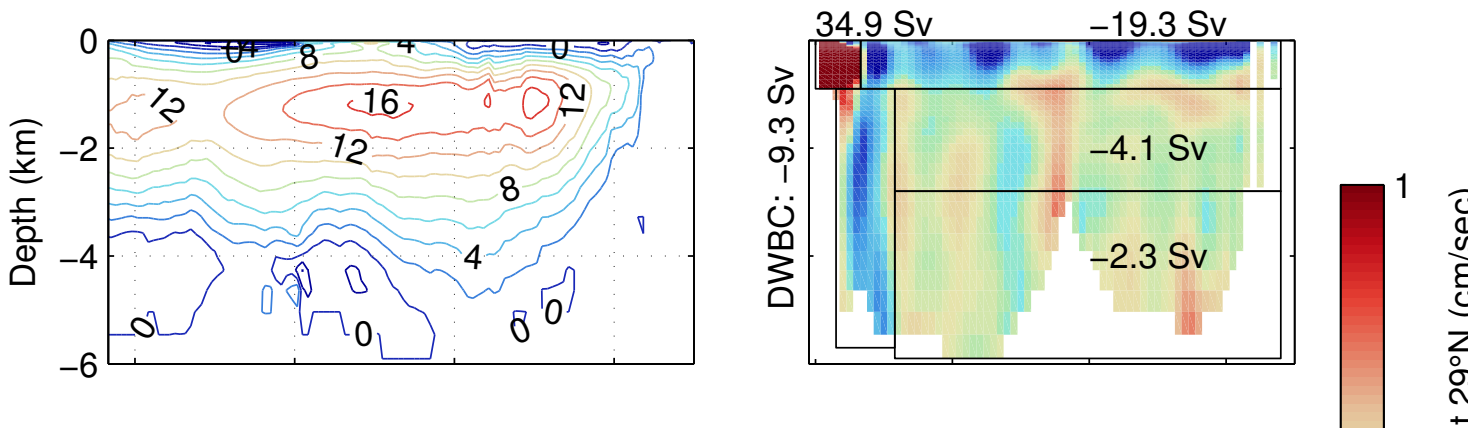

Modern_TS MOC and transports at $29^{\circ} \mathrm{N}$
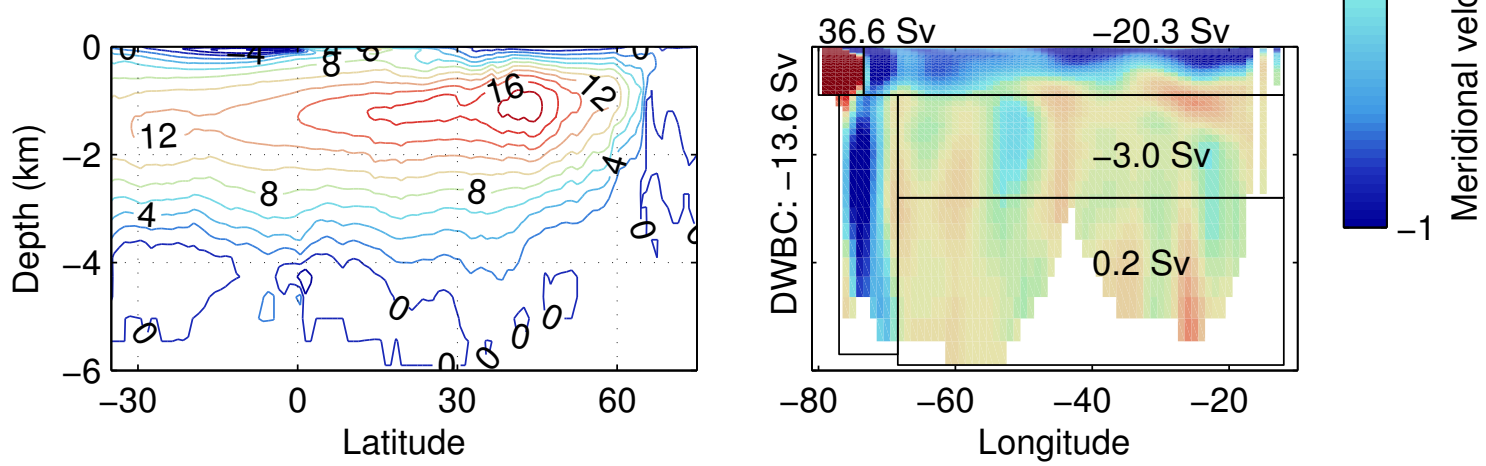

Figure 3-10: Annual mean meridional streamfunction in Sv (left) and meridional velocities at $29^{\circ} \mathrm{N}$ (right) for OCCA (top row) and Modern_TS (bottom row). Zero-line not shown in MOC plot for clarity. Net transports across $29^{\circ} \mathrm{N}$ are shown on the right for the Gulf Stream, DWBC, and upper, middle and deep interior flows. 

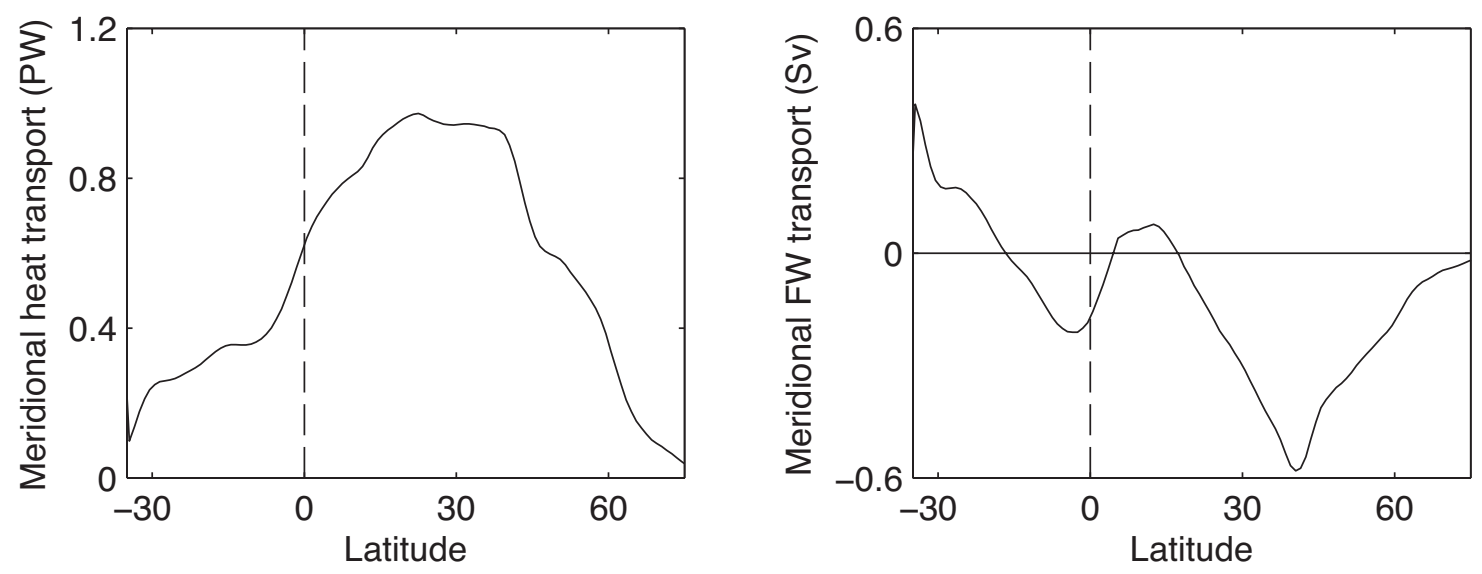

Figure 3-11: Meridional heat (left) and freshwater (right) transport in Modern_TS. Positive values imply northward transport of heat or freshwater.

location that is likely to experience increased flow due to transport east of the Bahamas and from recirculation. Given these differences, the Gulf Stream transport of both OCCA (35 Sv) and Modern_TS (37 Sv) appear reasonable. Meinen et al. [2006] compute DWBC transport as the integrated transport between 800 and $4800 \mathrm{dbar}$ from the shelf to $72^{\circ} \mathrm{W}$; they find a total transport of $-39 \pm 17 \mathrm{~Sv}$. Noting that much of the DWBC flow recirculates locally, the authors compute a net through-flow of $-11 \pm 18 \mathrm{~Sv}$. A strong recirculation in the depth range of the DWBC is not seen in the Modern_TS or OCCA velocity fields. However, the net throughflow of Modern_TS and OCCA are in agreement with those of Meinen et al. [2006].

Figure 3-11 shows the net heat and freshwater transported by the ocean at each latitude of the Modern_TS estimate. The Modern_TS heat transport is consistent with the conventional view that Atlantic heat transport is northward at all latitudes and peaks at about $1 \mathrm{PW}$ around $10-30^{\circ} \mathrm{N}$ [Ganachaud and Wunsch, 2003]. Oceanic freshwater transport closes the freshwater budget remaining from the processes of evaporation, precipitation and runoff (see Schanze et al. [2010] for a review). Roberts et al. [2004] compare Atlantic Ocean freshwater transport in the HadCEM and HadCM3 models with the transports inferred from a variety of observational studies (their figure 12). The Modern_TS transport is consistent 
with the conventional view summarized by Roberts et al. [2004]: the Atlantic Ocean loses freshwater to the atmosphere in the subtropical gyres (a negative slope in Figure 3-11) and gains it from the atmosphere in the inter-tropical convergence zone and in the N. Atlantic subpolar gyre (a positive slope in Figure 3-11).

Sea ice. Figure 3-12 shows February and August mean sea ice fraction in Modern_TS. As can be seen by comparing Figure 3-12 with a modern climatology (Figure 3-13), the Modern_TS distributions are close to modern ones. There is no August sea ice along the northeast Greenland coast in Modern_TS, while there is a small amount in the modern climatology. In today's ocean, cold, fresh waters of Arctic origin reach this region via the East Greenland Current, bringing with them sea ice exported from the Arctic; the imposition of a solid wall at $80^{\circ} \mathrm{N}$ in the model cuts off these inputs, making it difficult to maintain temperatures and sea ice distributions similar to real-world conditions.

\subsection{Modern state estimation with $\delta^{13} \mathbf{C}_{\text {DIC }}$ and $\delta^{18} \mathbf{O}_{\text {water }}$ observations}

As noted in Chapter $2, \delta^{18} \mathrm{O}_{\text {calcite }}$ and $\delta^{13} \mathrm{C}_{\text {calcite }}$ records from benthic foraminifera are key proxies that can help constrain deep water mass distributions and transport pathways; these proxies will be used in LGM state estimates presented in Chapter 5. As the MIT GCM does not include an isotope model, extensions were required to enable use of these proxies. The most important part of these extensions is a simple approach to modeling (1) the $\delta^{18} \mathrm{O}$ of seawater $\left(\delta^{18} \mathrm{O}_{\text {water }}\right)$, an important contributor to $\delta^{18} \mathrm{O}_{\text {calcite }}$, and (2) the $\delta^{13} \mathrm{C}$ of dissolved inorganic carbon $\left(\delta^{13} \mathrm{C}_{\mathrm{DIC}}\right)$, an important contributor to $\delta^{13} \mathrm{C}_{\text {calcite }}$. In this section, our approach to modeling $\delta^{18} \mathrm{O}_{\text {water }}$ and $\delta^{13} \mathrm{C}_{\mathrm{DIC}}$ is presented and then tested using modern water

column measurements of $\delta^{13} \mathrm{C}_{\text {DIC }}$ and $\delta^{18} \mathrm{O}_{\text {water }}$. Although coretop records of $\delta^{13} \mathrm{C}_{\text {calcite }}$ and $\delta^{18} \mathrm{O}_{\text {calcite }}$ are available [Marchal and Curry, 2008], they are difficult to interpret as they may be more representative of the late Holocene than of the modern ocean; coretop $\delta^{13} \mathrm{C}_{\text {calcite }}$ and $\delta^{18} \mathrm{O}_{\text {calcite }}$ records are therefore not used. 


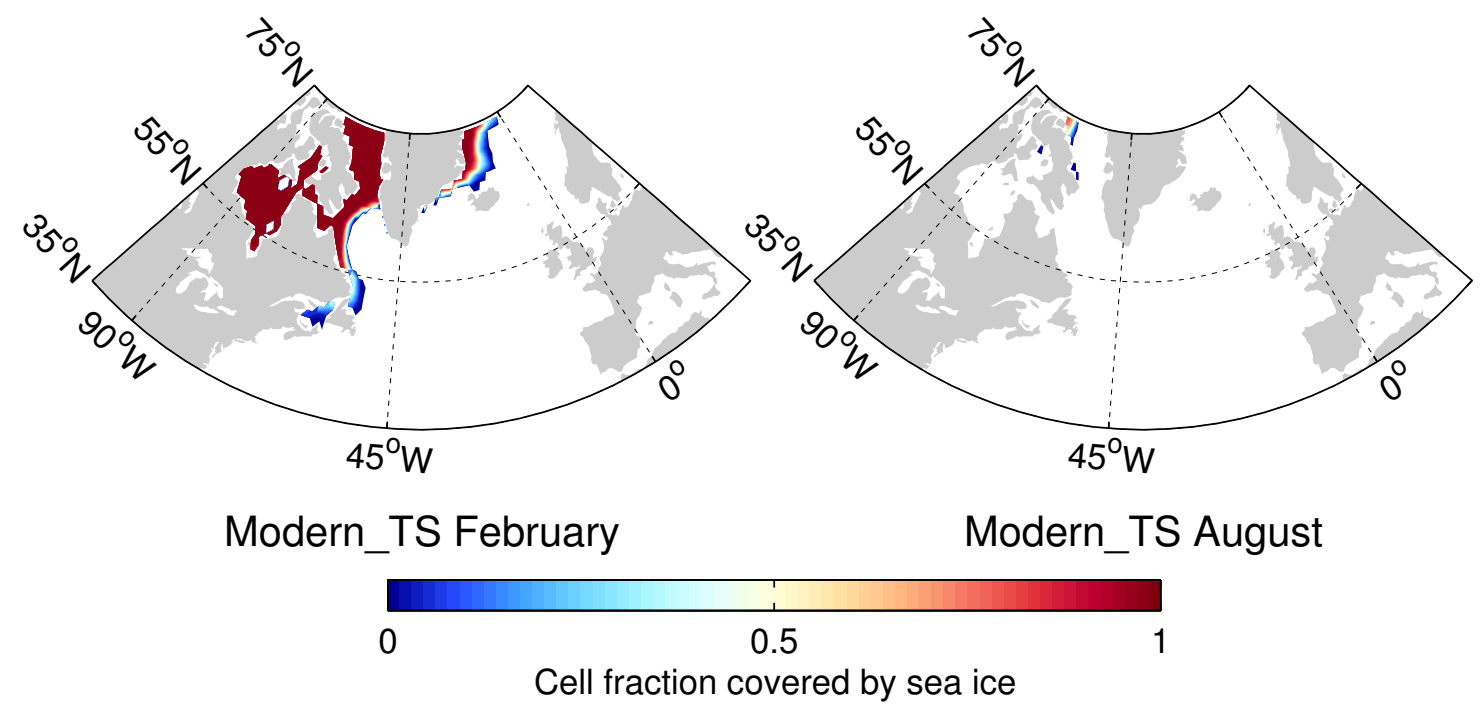

Figure 3-12: Fraction of grid cell covered by sea ice for February (left) and August (right) in Modern_TS.

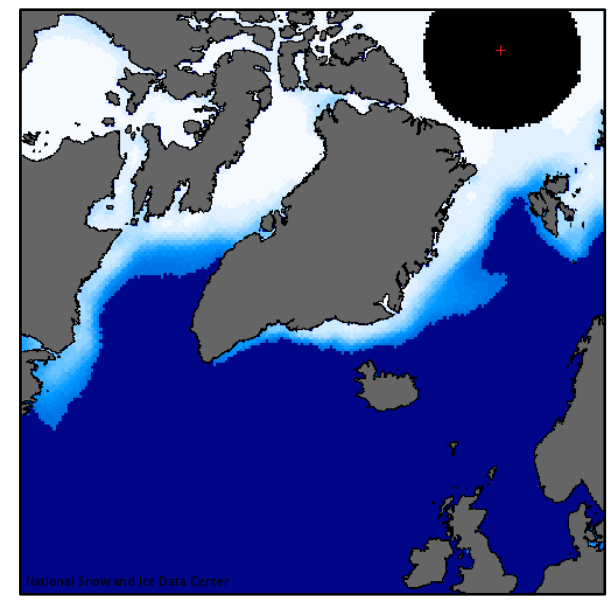

February sea ice concentration

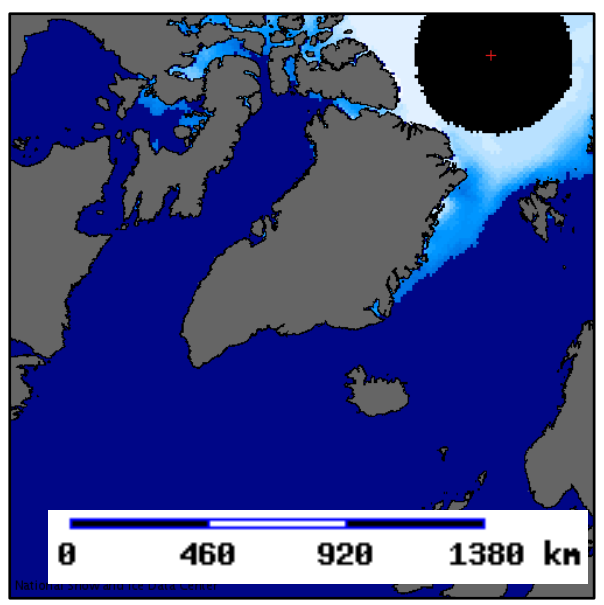

August sea ice concentration

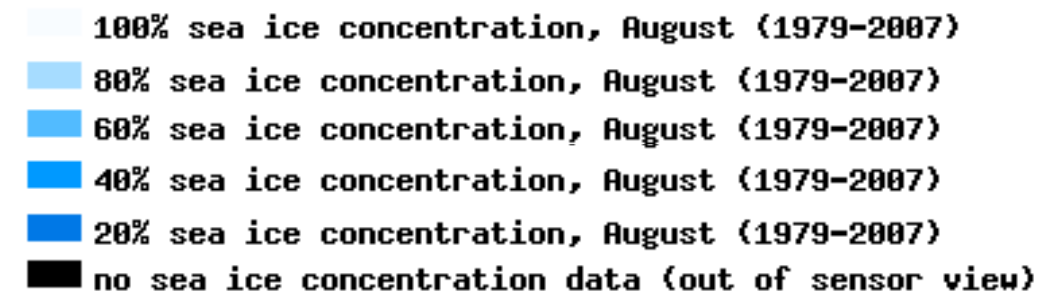

Figure 3-13: Climatology of sea ice concentration from 1979-2007 for February (left) and August (right). Subfigures from Maurer [2007]. 


\subsection{1 $\delta^{18} \mathbf{O}_{\text {water }}$ : forward modeling and state estimation}

Section 2.2.3 provided background on the definition of $\delta^{18} \mathrm{O}$, the main factors influencing the distribution of $\delta^{18} \mathrm{O}_{\text {water }}$ in the ocean, and the use of $\delta^{18} \mathrm{O}$ measurements in calcite as a proxy record. The goal here is to use $\delta^{18} \mathrm{O}_{\text {water }}$ as a water mass signature to constrain deep water mass distributions and pathways. For this purpose, the details of air-sea exchange and other surface ocean processes are not fundamental; instead, the focus is on the impact of advection and diffusion on the $\delta^{18} \mathrm{O}_{\text {water }}$ signature of water masses after they are isolated from the surface ocean. In this context, $\delta^{18} \mathrm{O}_{\text {water }}$ is a conservative property whose signature is set in the surface ocean.

$\delta^{18} \mathrm{O}_{\text {water }}$ is modeled as a passive, conservative tracer that is initialized with an estimate of the modern $\delta^{18} \mathrm{O}_{\text {water }}$ distribution; the surface ocean $\delta^{18} \mathrm{O}_{\text {water }}$ value is imposed using a Dirichlet boundary condition (concentration in surface waters is fixed); $\delta^{18} \mathrm{O}_{\text {water }}$ at the southern boundary are also imposed with a Dirichlet boundary condition. Surface ocean $\delta^{18} \mathrm{O}_{\text {water }}$ levels are highly dependent on the volume flux and isotopic signature of freshwater sources and have a strong seasonal cycle. Given uncertainties in these processes, $\delta^{18} \mathrm{O}$ data are not considered above a depth of $135 \mathrm{~m}$, and the effect of processes in the top 135 $\mathrm{m}$ on the deeper ocean are represented with a concentration boundary condition applied in the model at a depth of $135 \mathrm{~m}$. Boundary conditions on $\delta^{18} \mathrm{O}_{\text {water }}$ are fixed in time; seasonal variation is not considered. A gridded estimate of modern mean $\delta^{18} \mathrm{O}_{\text {water }}$ [LeGrande and Schmidt, 2006] is used as the first guess initial and boundary conditions.

To permit $\delta^{18} \mathrm{O}_{\text {water }}$ data constraints, the Modern_TS cost function (Equation 3.4) is augmented to include $\delta^{18} \mathrm{O}_{\text {water }}$ model-data misfit terms:

$$
\begin{gathered}
J_{\text {data, },{ }^{18} \mathbf{O}_{\text {water }}}=m u l t \cdot \frac{2}{n y} \sum_{y=n y / 2}^{n y}\left(\overline{\boldsymbol{\delta}^{\mathbf{1 8}} \mathbf{O}_{\text {water,mod }}(y)}-\boldsymbol{\delta}^{18} \mathbf{O}_{\text {water,obs }}\right)^{T} \\
\mathbf{W}_{\boldsymbol{\delta}^{18} \mathbf{O}_{\text {water }}}\left(\overline{\boldsymbol{\delta}^{\mathbf{1 8}} \mathbf{O}_{\text {water,mod }}(y)}-\boldsymbol{\delta}^{\mathbf{1 8}} \mathbf{O}_{\text {water,obs }}\right)
\end{gathered}
$$

where mult is a balancing factor described in the next paragraph, $\overline{\boldsymbol{\delta}^{\mathbf{1 8}} \mathbf{O}_{\text {water,mod }}(y)}$ is the 
modeled annual mean tracer signatures for year $y, \delta^{18} \mathbf{O}_{\text {water,obs }}$ is the observations, and $\mathbf{W}_{\boldsymbol{\delta}^{\mathbf{1 8}} \mathbf{O}_{\text {water }}}$ is the weighting matrix.

Observations are taken from the database of Schmidt et al. [1999] - the most comprehensive database of modern $\delta^{18} \mathrm{O}_{\text {water }}$ measurements available. A variety of flags are used in this database to note potential concerns. Of the 14 defined flag types, 6 flag types are identified as too serious for present purposes and those data are excluded from analysis. These records were flagged because the data were sign corrected or rescaled by the database authors after publication (flag C), sub-datasets were re-calibrated to ensure deep water mass properties agree (flags $\mathrm{G}, \mathrm{H}, \mathrm{I}, \mathrm{J}$ ), or data were identified as likely to be heavily influenced by nearshore freshwater input (flag L). After exclusion of flagged data, there are 1,928 $\delta^{18} \mathrm{O}_{\text {water }}$ records in our model domain; Figure 3-14 (upper plot) shows the distribution of the remaining observations with depth and latitude.

A variety of uncertainties impact how well these data can be represented with a model: sampling error, laboratory analysis error, time variation in $\delta^{18} \mathrm{O}_{\text {water }}$, and errors due to oversimplification of complex air-sea interactions and freshwater inputs with a Dirichlet boundary condition. An uncertainty of $0.15 \%$ is assigned here for data below a depth of $510 \mathrm{~m}$, $0.3 \%$ is assigned between the depths of 510 and $135 \mathrm{~m}$, and data above $135 \mathrm{~m}$ are excluded from consideration. Histograms of observations in these depth ranges are compared with these assumed uncertainties in the lower plots of Figure 3-14; the assumed uncertainties are much smaller than the dynamic range in the data for both data between 135 and $510 \mathrm{~m}$ and for data below $510 \mathrm{~m}$.

The extended cost function includes $3 \times 10^{6}$ data elements from the gridded OCCA temperature and salinity data and 1,928 point measurement $\delta^{18} \mathrm{O}_{\text {water }}$ records. This mismatch in number of data constraints implies a stronger emphasis on OCCA by a factor of 1600 , a relative weighting that is not representative of the information contained in each dataset. The OCCA T/S data do not all provide independent constraints on the model, particularly given our requirement for relatively large-scale atmospheric adjustments. A rough calculation is used to downweight the T/S data relative to the isotope data: assuming 

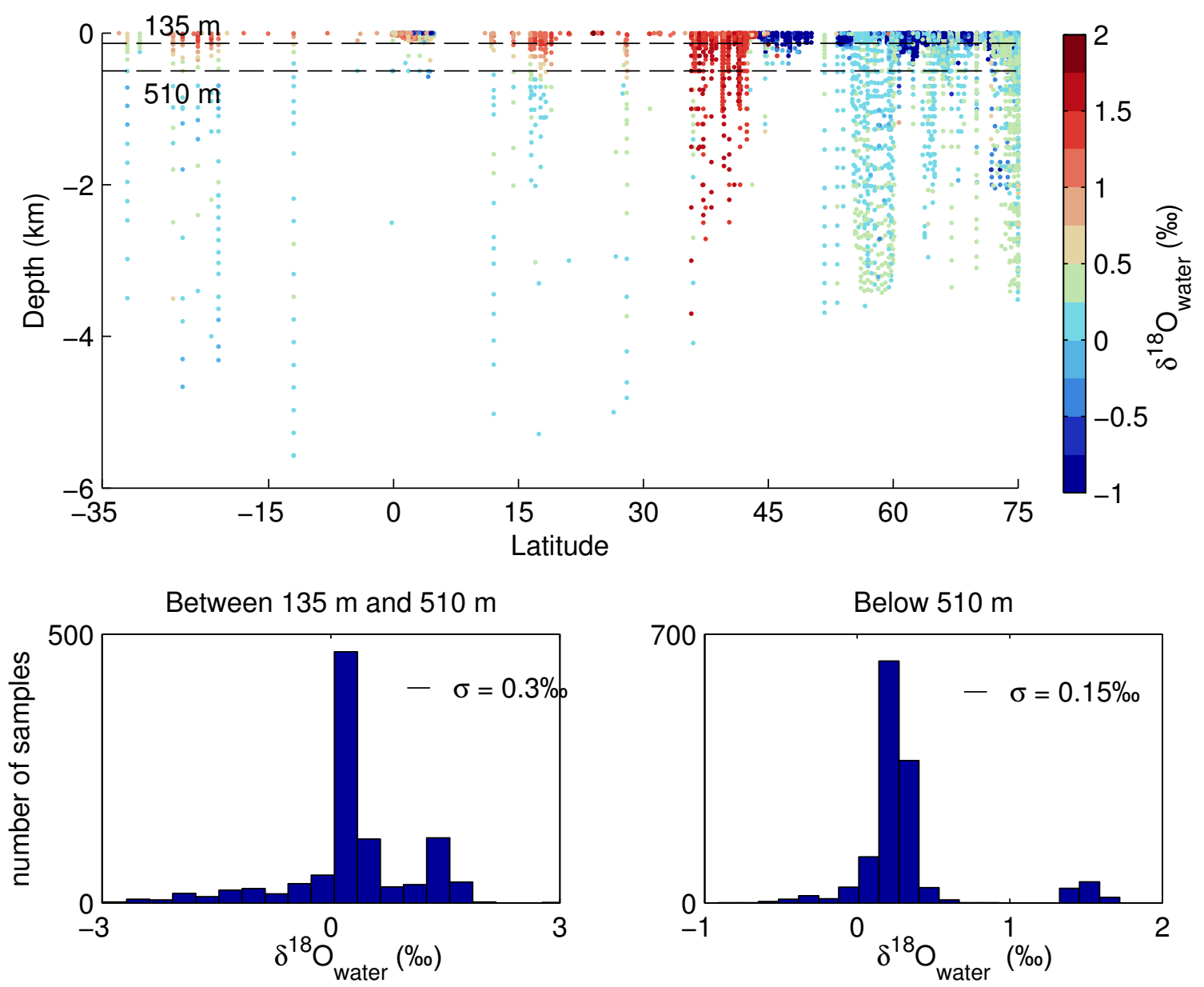

Figure 3-14: The Schmidt et al. [1999] $\delta^{18} \mathrm{O}$ observations used in this thesis plotted against latitude and depth (top plot, data above $135 \mathrm{~m}$ are excluded from consideration). Histograms are shown on the bottom left for samples collected between 135 and $510 \mathrm{~m}$ water depth and on the bottom right for samples collected below $510 \mathrm{~m}$ water depth. Horizontal lines show assumed uncertainties of $0.3 \%$ and $0.15 \%$; the dynamic range of the data is much larger than assumed uncertainties.

covariance scales for both temperature and salinity of $300 \mathrm{~km}$ in the horizontal and $100 \mathrm{~m}$ in the vertical implies on the order of every 3rd model cell is independent in the horizontal and in the vertical for a downweighting factor of $3^{3}=27$. These assumed length scales are chosen to be conservative so that the downweighting factor is unlikely to be too large. In keeping with our emphasis on a cost function normalized to one full year, and assuming deep ocean T/S data at monthly frequency deserves the same emphasis as deep ocean tracer 
data sampled at an annual rate, a factor of 12 is included to compute a final downweighting of OCCA T/S data relative to the isotopic data of $27 \times 12=324$. For convenience, this factor is included as the mult term in Equation 3.6.

Model initial and boundary conditions for $\delta^{18} \mathrm{O}_{\text {water }}$ are uncertain and improvements in these parameters have the potential to significantly improve the model fit to the $\delta^{18} \mathrm{O}_{\text {water }}$ measurements. The model is thus augmented with new control variables for $\delta^{18} \mathrm{O}_{\text {water }}$ initial conditions, southern boundary conditions, and surface concentration boundary conditions. $J_{c t r l}$ is augmented so that adjustments to these new control variables are penalized. Table 3.3 shows the full list of control variables; these will be used throughout the rest of the thesis.

$\underline{\text { Initial conditions (XYZ) }}$

$\begin{array}{ll}\text { Temperature } & { }^{\circ} \mathrm{C} \\ \text { Salinity } & \\ \delta^{18} \mathrm{O}_{\text {water }} & \% 0 \\ \delta^{13} \mathrm{C}_{\text {DIC }} & \% 0\end{array}$

Atmospheric forcing (XYT)

Shortwave radiation

2-m air temperature

$10-\mathrm{m}$ zonal wind speed

10-m meridional wind speed

2-m Specific humidity

Precipitation

\begin{tabular}{lll} 
& \multicolumn{2}{c}{ Surface B.C. (XY) } \\
\cline { 2 - 2 }${ }^{\circ} C$ & $\delta^{18} \mathrm{O}_{\text {water }}$ & $\% 0$ \\
$\delta^{13} \mathrm{C}_{\text {DIC }}$ & $\% 0$ \\
& & \\
$\% 0$ & &
\end{tabular}

$\frac{\text { Southern B.C. }(\mathrm{XZT})}{\text { Temperature }{ }^{\circ} \mathrm{C}}$

Salinity

U velocity $\mathrm{m} / \mathrm{s}$

$\mathrm{V}$ velocity $\mathrm{m} / \mathrm{s}$

$\delta^{18} \mathrm{O}_{\text {water }} \quad \%$

$\delta^{13} \mathrm{C}_{\text {DIC }} \quad \%$

Table 3.3: A full list of control variables used in the thesis. Codes in parenthesis are defined as follows: $\mathrm{XYZ}$ indicates whether the control variables span longitude, latitude, and/or depth and $\mathrm{T}$ is appended for controls that are permitted to vary on a monthly basis (no $\mathrm{T}$ indicates the control variables are initial conditions or are fixed in time).

\subsection{2 $\delta^{13} \mathbf{C}_{\text {DIC }}$ : forward modeling and state estimation}

Section 2.2.3 provided background on $\delta^{13} \mathrm{C}$, the importance of remineralization in its distribution, and its use as a proxy for paleoceanographic studies. This thesis considers $\delta^{13} \mathrm{C}_{\text {DIC }}$ 
below $1000 \mathrm{~m}$ only: the impact of remineralization on $\delta^{13} \mathrm{C}_{\mathrm{DIC}}$ above $1000 \mathrm{~m}$ is expected to be large but is poorly understood, and only a small number of LGM sediment core $\delta^{13} \mathrm{C}$ records fall above $1000 \mathrm{~m}$. Also, the modeling approach for $\delta^{13} \mathrm{C}_{\text {calcite }}$ of Marchal and Curry [2008] is a useful base for the model used here, but that approach has been developed for, and tested with, a deep-ocean model that starts at $1000 \mathrm{~m}$. As was done by Marchal and Curry [2008], $\delta^{13} \mathrm{C}_{\mathrm{DIC}}$ is used here to constrain deep water mass distributions and pathways. For this purpose, it is assumed that $\delta^{13} \mathrm{C}_{\mathrm{DIC}}$ can be adequately modeled by considering the effects of remineralization, ocean mixing, and transport processes [Marchal and Curry, 2008].

Since the remineralization rate deeper than $1000 \mathrm{~m}$ is small [Aristegui et al., 2004] and Atlantic waters are relatively recently ventilated [Wunsch and Heimbach, 2008], it can be expected that the Atlantic $\delta^{13} \mathrm{C}_{\text {DIC }}$ distribution below $1000 \mathrm{~m}$ is mostly dominated by surface end-member values and ocean mixing and transport processes, rather than by the influence of remineralization [Marchal and Curry, 2008]. Thus, while including the impact of remineralization is important, a simple approximation appears to be sufficient. Marchal and Curry [2008] assume that, at leading order, advection dominates diffusion in controlling the $\delta^{13} \mathrm{C}_{\mathrm{DIC}}$ distribution and that, in terms of concentrations, ${ }^{12} \mathrm{C}+{ }^{13} \mathrm{C} \approx{ }^{12} \mathrm{C}$ (since ${ }^{12} \mathrm{C}$ constitutes about $99 \%$ of natural carbon). The transport of $\delta^{13} \mathrm{C}_{\mathrm{DIC}}$ can then be described by equation 14 of Marchal and Curry [2008], reproduced here for completeness:

$$
\mathbf{u} \cdot \nabla \delta^{13} \mathrm{C}_{\mathrm{DIC}}=\frac{1}{\lambda}\left(\delta^{13} \mathrm{C}_{\mathrm{org}}-\delta^{13} \mathrm{C}_{\mathrm{DIC}}\right)+\epsilon
$$

where $\mathbf{u}$ is the three-dimensional vector velocity, $\lambda$ is a relaxation timescale, $\delta^{13} \mathrm{C}_{\text {org }}$ is the $\delta^{13} \mathrm{C}$ of organic matter, $\delta^{13} \mathrm{C}_{\mathrm{DIC}}$ is the current model value, and $\epsilon$ accounts for (1) uncertainty in $\delta^{13} \mathrm{C}_{\text {org }}$ and $\lambda$ and (2) errors associated with neglecting the effect of mixing and time-dependency. The effect of remineralization takes the form of a relaxation term because $\delta^{13} \mathrm{C}$ is a ratio of carbon isotope concentrations; the effect of remineralization on a standard concentration like phosphate would take the form of a direct source term. The relaxation timescale is obtained from $\lambda=C / J$ where $C$ is the concentration of total 
dissolved inorganic carbon in seawater and $J$ is the rate of production of inorganic carbon by remineralization of organic carbon (unrelated to cost function $J$ ). Constant values $\frac{1}{\lambda}=0.6 \cdot 10^{-4} \mathrm{yr}^{-1}$ and $\delta^{13} \mathrm{C}_{\text {org }}=-20 \%$ are assumed (see Marchal and Curry [2008] for full details). Note that the estimate of $\frac{1}{\lambda}$ used here is only applicable for the deep ocean; this estimate is sufficient here as we do not consider data above $1000 \mathrm{~m}$.

With the exception of the added remineralization term, the treatment of $\delta^{13} \mathrm{C}_{\mathrm{DIC}}$ in the MIT GCM and state estimation framework follows that described in Section 3.4.1 for $\delta^{18} \mathrm{O}_{\text {water }}$. The surface ocean boundary condition is applied above $1000 \mathrm{~m}$ for $\delta^{13} \mathrm{C}_{\mathrm{DIC}}$. No gridded $\delta^{13} \mathrm{C}_{\mathrm{DIC}}$ products were available as first guess initial and boundary conditions; instead, GEOSECS $\delta^{13} \mathrm{C}_{\mathrm{DIC}}$ measurements ${ }^{2}$ were used to guide these first guess estimates. The initial interior $\delta^{13} \mathrm{C}_{\mathrm{DIC}}$ is set to $0.8 \%$, the $1000 \mathrm{~m}$ boundary condition is set to $0.7 \%$, and the southern boundary condition is set to $0.6 \%$. These values are similar because the modern $\delta^{13} \mathrm{C}_{\mathrm{DIC}}$ distribution is itself relatively uniform.

As was done for $\delta^{18} \mathrm{O}_{\text {water }}$, the Modern_TS cost function is augmented to include modeldata misfit terms for $\delta^{13} \mathrm{C}_{\mathrm{DIC}}$ :

$$
\begin{gathered}
J_{d a t a, \delta^{13} C_{D I C}}=\text { mult } \cdot \frac{2}{n y} \cdot \sum_{y=n y / 2}^{n y}\left(\overline{\boldsymbol{\delta}^{13} \mathbf{C}_{\text {DIC,mod }}(y)}-\boldsymbol{\delta}^{\mathbf{1 3}} \mathbf{C}_{\mathbf{D I C}, \mathbf{o b s}}\right)^{T} . \\
\mathbf{W}_{\boldsymbol{\delta}^{13} \mathbf{C}_{\mathbf{D I C}}} \cdot\left(\overline{\boldsymbol{\delta}^{\mathbf{1 3}} \mathbf{C}_{\mathbf{D I C}, \mathbf{m o d}}(y)}-\boldsymbol{\delta}^{\mathbf{1 3}} \mathbf{C}_{\mathbf{D I C}, \mathbf{b b s}}\right),
\end{gathered}
$$

where mult is the same balancing factor described for Equation 3.8, $\overline{\boldsymbol{\delta}^{13} \mathbf{C}_{\mathbf{D I C}, \bmod }(y)}$ are the modeled annual mean tracer signatures for year $y, \boldsymbol{\delta}^{\mathbf{1 3}} \mathbf{C}_{\mathbf{D I C}, \text { obs }}$ are GEOSECS measurements of water-column $\delta^{13} \mathrm{C}_{\mathrm{DIC}}$, and $\mathbf{W}_{\delta^{13} \mathrm{C}_{\text {DIC }}}$ is the weighting matrix. There are 431 GEOSECS observations in the domain of our model [Kroopnick, 1985]. Figure 3-15 (top plot) shows the distribution of these observations with latitude and depth. Although large numbers of water-column $\delta^{13} \mathrm{C}_{\text {DIC }}$ data are available from the GLODAP [Key et al., 2004] and CARINA [Key et al., 2010] databases, these data were collected more recently than

\footnotetext{
${ }^{2}$ Data obtained from http://iridl.ldeo.columbia.edu/SOURCES/.GEOSECS. These data have been corrected by Kroopnick [1985] for incorrect sample handling on deck.
} 

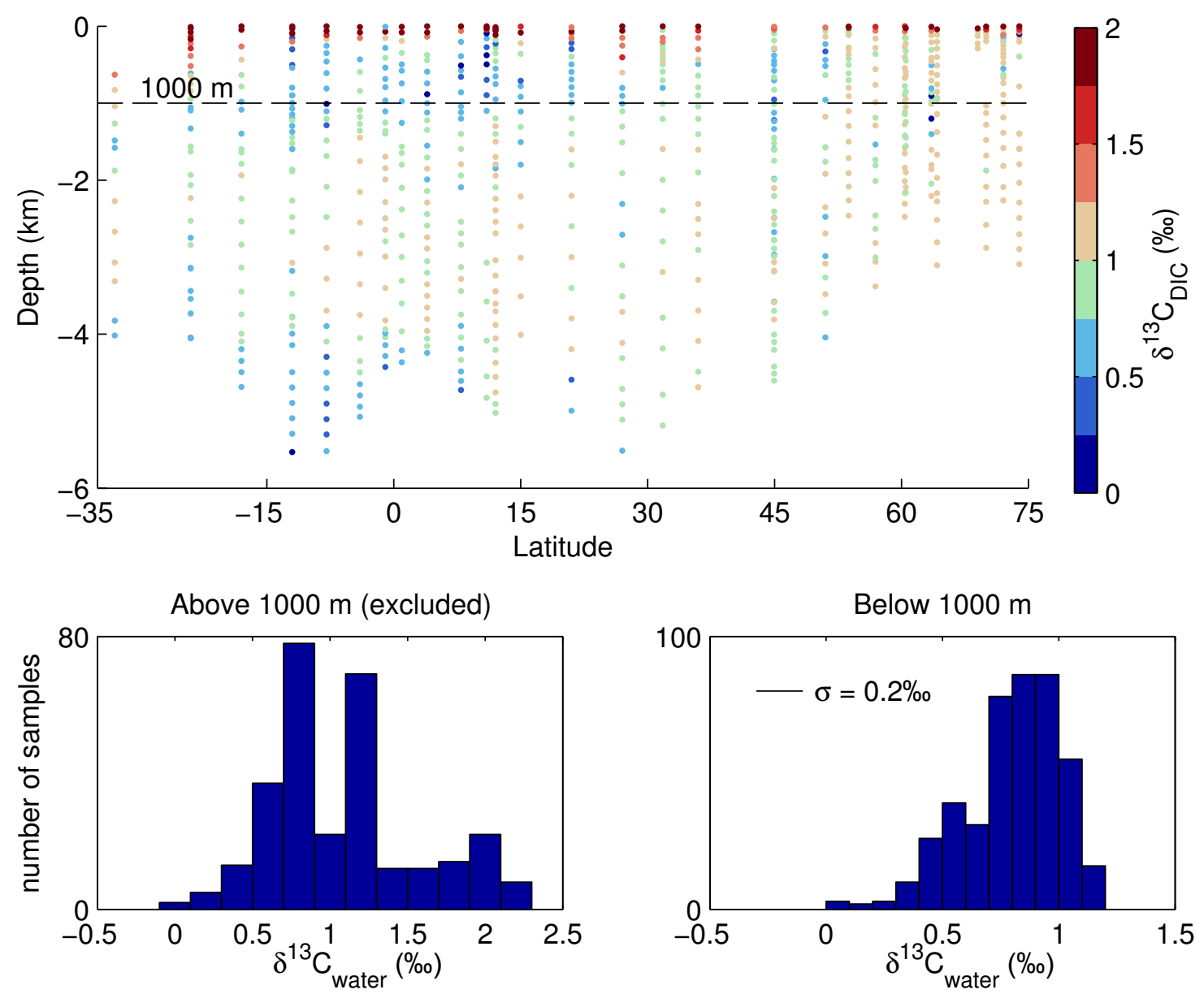

Figure 3-15: The GEOSECS $\delta^{13} \mathrm{C}_{\text {DIC }}$ observations used in this thesis plotted against latitude and depth (top plot, data above $1000 \mathrm{~m}$ are excluded from consideration). Histograms are shown on the bottom left for samples collected above $1000 \mathrm{~m}$ water depth and on the bottom right for samples collected below $1000 \mathrm{~m}$ water depth. The horizontal line on the bottom right shows the assumed uncertainties of $0.2 \%$ for comparison.

the GEOSECS measurements, and thus are likely to have been more significantly affected by the impact of anthropogenic $\mathrm{CO}_{2}$ with its low ${ }^{13} \mathrm{C} /{ }^{12} \mathrm{C}$ ratio (see Quay et al. 2007 for an Atlantic-focused discussion of this effect). Anthropogenic impacts are inconsistent with the steady state assumptions used in this thesis. An uncertainty of $0.2 \%$ is assigned below $1000 \mathrm{~m}$ (data above $1000 \mathrm{~m}$ are excluded from the state estimate); the weighting matrix, $\mathbf{W}_{\boldsymbol{\delta}^{13} \mathbf{C}_{\text {DIC }}}$, thus contains a value of $1 / 0.2^{2}$ on all diagonals, and a value of 0 on all off- 
diagonal elements (the data are assumed independent). Although instrumental precision for measuring $\delta^{13} \mathrm{C}$ is much better than $0.2 \%$ (see Section 2.2.3), this larger uncertainty is chosen to accommodate uncertainties in the remineralization formulation. The assumed uncertainty is compared with the dynamic range in the data in the lower right hand plot of Figure 3-15.

The model is augmented with new control variables for $\delta^{13} \mathrm{C}_{\text {DIC }}$ initial and boundary conditions (see Table 3.3), and $\mathbf{J}_{c t r l}$ is modified to penalize adjustments to these control variables.

\subsubsection{The Modern_Iso estimate}

As a first test of the isotope modeling approaches described above, the Modern_TS configuration is extended to include $\delta^{18} \mathrm{O}_{\text {water }}$ and $\delta^{13} \mathrm{C}_{\text {DIC }}$. The cost function is applied to the second year of a two year state estimate. A total of 32 iterations were used to reduce the total cost of the misfit to $\mathrm{T} / \mathrm{S} / \delta^{13} \mathrm{C}_{\mathrm{DIC}} / \delta^{18} \mathrm{O}_{\text {water }}$ from $1.2 \times 10^{7}$ to $2.3 \times 10^{6}$, a reduction of $81 \%$. Cost was still going down slowly after 32 iterations, but the solution at iteration 32 is statistically acceptable (see below) so further optimization is not warranted. The iteration 32 solution is hereafter called the "Modern_Iso" estimate.

The mean cost for each data type in Modern_Iso is 0.32 for temperature, 0.26 for salinity, 0.43 for $\delta^{13} \mathrm{C}_{\text {DIC }}$ and 0.63 for $\delta^{18} \mathrm{O}_{\text {water }}$. These costs are only $13 \%$ (4\%) higher for temperature (salinity) than those obtained for the Modern_TS estimate, which was constrained by only temperature and salinity data. This indicates that large changes to the model temperature and salinity distributions were not required to fit the $\delta^{13} \mathrm{C}_{\mathrm{DIC}}$ and $\delta^{18} \mathrm{O}_{\text {water }}$ data. Cumulative distribution functions (CDFs) were examined for the fit of Modern_Iso to the $\mathrm{T} / \mathrm{S}$ data (not shown); the distributions are nearly the same as those shown in Figure 3-2 for Modern_TS, another indication that the T/S distributions in the Modern_Iso solution are not significantly modified from those of Modern_TS. The CDFs of the fit of the model to the isotope data (Figure 3-16) indicate a statistically satisfactory solution: $97 \%$ (99\%) of the modeled $\delta^{18} \mathrm{O}\left(\delta^{13} \mathrm{C}\right)$ values are within $2 \sigma$ of the observations. 

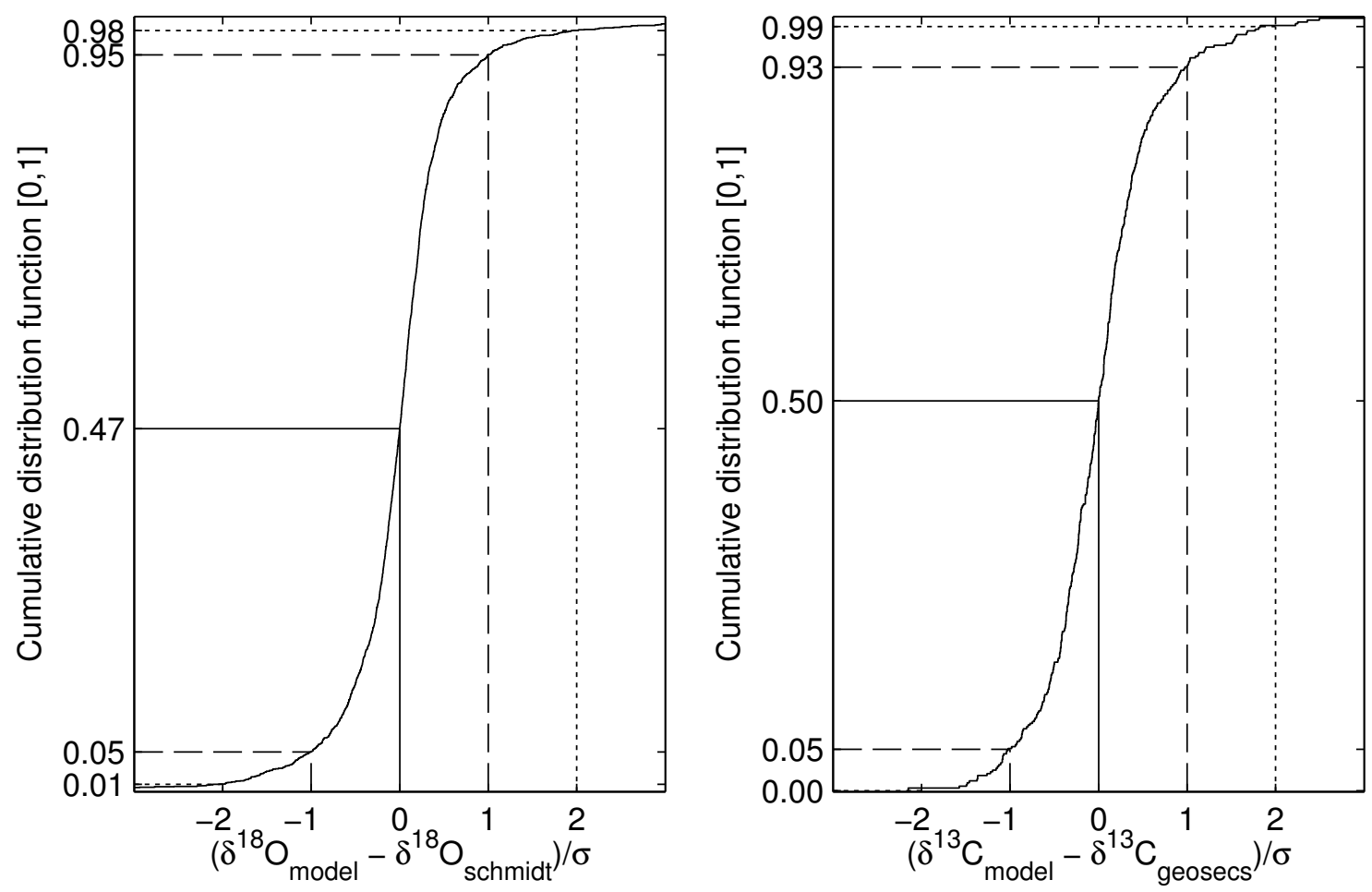

Figure 3-16: CDFs for signed, normalized misfits of Modern_Iso $\delta^{18} \mathrm{O}_{\text {water }}$ (left) and $\delta^{13} \mathrm{C}_{\text {DIC }}$ values (right). The solution fits the Schmidt et al. [1999] $\delta^{18} \mathrm{O}_{\text {water }}$ and GEOSECS $\delta^{13} \mathrm{C}_{\text {DIC }}$ data closely.

Consider how the state estimate improves the agreement between model and data. The approach used in this thesis depends on the model integration to compute the forward state and the adjoint sensitivities. The physical connection between a given data point and the control parameters is limited to the physical distance over which advection and mixing processes act in the period simulated in the state estimate. Accumulation of sensitivities to compute control adjustments can be thought of as a process akin to backtracking particle trajectories, except the full fluid behavior including mixing is considered. Since the data are below $135 \mathrm{~m}\left(\delta^{18} \mathrm{O}\right)$ or $1000 \mathrm{~m}\left(\delta^{13} \mathrm{C}\right)$ and the state estimate duration is short relative to deep ocean advection and mixing timescales, most of the data are connected by fluid motion to only a small region near the data location. In these conditions, tracer initial condition adjustments provide much of the improvement in fit to the data. Figure 3-17 shows that the 


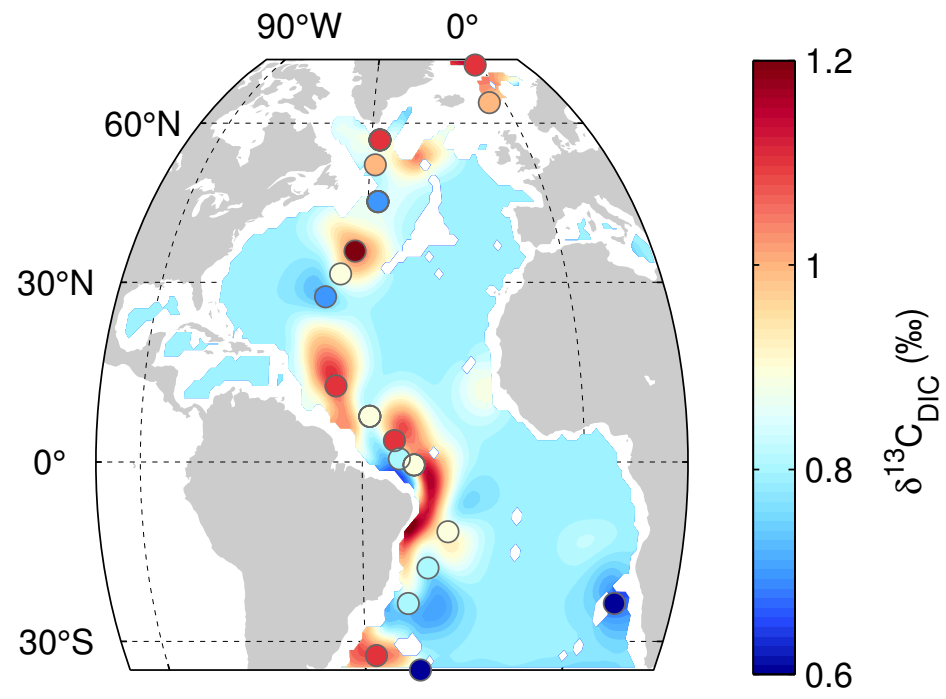

Figure 3-17: The Modern_Iso $\delta^{13} \mathrm{C}_{\text {DIC }}$ distribution at a model depth of $2730 \mathrm{~m}$ (colored contours) compares well with GEOSECS $\delta^{13} \mathrm{C}_{\text {DIC }}$ observations from a similar depth (colored circles, data from depths 2605 to $2854 \mathrm{~m}$ ). At this depth, the $\delta^{13} \mathrm{C}_{\text {DIC }}$ distribution should be smooth and large scale; instead, the distribution is unrealistically patchy in this 10 year state estimate.

resulting solution, while providing a good fit to the data, implies local, small-scale sources and sinks of $\delta^{13} \mathrm{C}_{\mathrm{DIC}}$ that are not realistic. Such small-scale patchiness could be removed by imposing large-scale smoothness constraints in the cost function, but such an approach may introduce physically unrealistic tracer distributions and is not taken here. Two observations can be made from the inclusion of $\delta^{18} \mathrm{O}_{\text {water }}$ and $\delta^{13} \mathrm{C}_{\mathrm{DIC}}$ data as constraints in the Modern_TS state estimate configuration: (1) it is relatively straightforward to obtain a statistically good fit to deep ocean tracer data in a short state estimate, and (2) without adding smoothing constraints (which may physically unrealistic), sparse tracer data are insufficient for constraining deep ocean circulation in decadal length state estimates. These observations motivate a search for longer state estimates, a goal that is pursued in the next section. 


\subsection{Extending state estimate length: the search for a sea- sonal steady state}

An equilibrium ocean estimate (EOE) is a term defined in this thesis to describe an estimate of ocean state that is consistent with (1) known dynamics, (2) available data given their uncertainties, and (3) a steady state assumption (whether fully steady or cyclic in nature). Chapter 1 introduced the potential benefits of EOE, Section 3.2.4 described aspects of our state estimation framework that are specifically adapted for EOE, and Section 3.4 provided a concrete example of the limitations of short state estimates to motivate the potential benefits of EOE. As we know of no ocean state estimates that satisfy these three goals simultaneously, it is not known whether an EOE is an achievable goal.

This section describes two approaches used in this thesis to extend the number of model years that can be included in an ocean state estimate while remaining as close as possible to a target data set. The effectiveness of these approaches, called asynchronous timestepping and carry-over control, is evaluated through a number of experiments; limitations of the approaches are identified. The work presented here represents a step towards EOE, and the section concludes with a discussion of avenues for future investigations.

\subsubsection{Asynchronous timestepping}

All state estimates presented earlier in the chapter employed synchronous timestepping. Asynchronous timestepping refers to the practice of timestepping the model tracer equations forward at a longer timestep than the momentum equations; see Danabasoglu [2004] for an evaluation of this approach for forward model integrations. Simulation performance is a key roadblock in achieving long-running ocean state estimates, and this approach can provide speedups of a factor of 10. Although asynchronous timestepping has been used previously in exploratory ocean state estimates (G. Forget, pers. comm.), to our knowledge a quantitative evaluation of the approach in state estimation has not previously been made. This section presents such an evaluation. 
The primary advantage of asynchronous timestepping is faster simulation times. The disadvantage is less accurate simulation of oceanic physics; in particular, modeled wave propagation speeds and speeds suffer relatively large errors. Waves are an important mechanism by which dynamical information propagates in the model (and in the ocean), and the adjoint relies on tracking their information propagation to infer dynamical control adjustments. The following test is employed to evaluate the extent to which these issues impact the state estimation configurations used in this thesis.

1. The model configuration used in the Modern_Iso state estimate is applied in a new 10 year simulation, this time run with asynchronous timestepping (synchronous timestepping was used for the Modern_Iso estimate). The resulting unadjusted model estimate will be referred to as the first guess async (this is a forward simulation).

2. The optimization process is then applied with asynchronous timestepping used in all iterations. At iteration 20, the optimization is stopped as the iteration 20 estimate is sufficiently improved. This iteration 20 estimate is referred to as the improved async estimate. This estimate has a good fit to the target data, but it is possible that the model physics are so heavily distorted that the fit to the data is achieved through control adjustments that would be meaningless in a model with more accurate physics. Such a result would invalidate asynchronous timestepping as a viable option for the state estimation configuration used here. The following step tests for this possibility.

3. The iteration 20 configuration (including the control variable adjustments inferred through asynchronous timestepping) is then repeated in a new simulation that uses synchronous timestepping; no other modifications to the configuration are made and no optimization is done in synchronous mode. The resulting estimate is referred to as the improved sync estimate.

There is a general philosophy in nonlinear optimization that any approach to finding a lower minimum of the cost function is acceptable: that the cost function is reduced is taken 
as evidence that the solution is an improvement (the cost function is assumed to be a reasonable measure of solution quality). The idea of the test outlined step three above is that if the improved sync estimate is indeed a reasonable solution, it implies that the control adjustments found through the optimization process are appropriate ones. That they were found via an asynchronous timestepping approach with inexact physics is not important. On the other hand, if the improved sync estimate is not a good solution (i.e. it has a high cost), the implication is that the errors associated with the asynchronous timestepping approach are too large, the inferred controls are not appropriate, and asynchronous timestepping cannot be applied.

The misfits of the first guess async, improved async, and improved sync estimates to OCCA temperature and salinity are now examined. Mean temperature and salinity costs are computed for each model layer in each month of the estimates (recall that cost $=$ $\left.(\text { model }- \text { data })^{2} / \sigma^{2}\right)$; the results are shown in Figure 3-18. The 1 st guess costs (top row) show that in the first years of the unadjusted estimate, the solution remains close to OCCA and costs are low. By year 10, costs have increased greatly: the model is drifting away from OCCA T/S (as expected for a forward model). After 20 iterations in the optimization process, the improved async estimate (Figure 3-18, middle row) has greatly reduced costs; costs are particularly low for years 6-10, the years that are included in the cost function. In fact, in order to obtain low costs in years 5-10, the optimization has worsened the fit in the early model years; this result is expected given that these years are not included in the cost function, but is an indication that model drift has not been eradicated.

A comparison of the middle and bottom rows of Figure 3-18 shows that costs are very similar for the improved async and improved sync estimates. While slight differences are visible, the improved sync estimate has acceptably low costs. A comparison of other model characteristics are also favorable. For example, the Gulf Stream/DWBC transports of the improved async estimate (35.0/-8.5 Sv) are similar to those of the improved sync estimate (34.1/-8.9 Sv); these transports are much more similar to each other than either is to the first guess async transports (37.9/-15.8 Sv). 

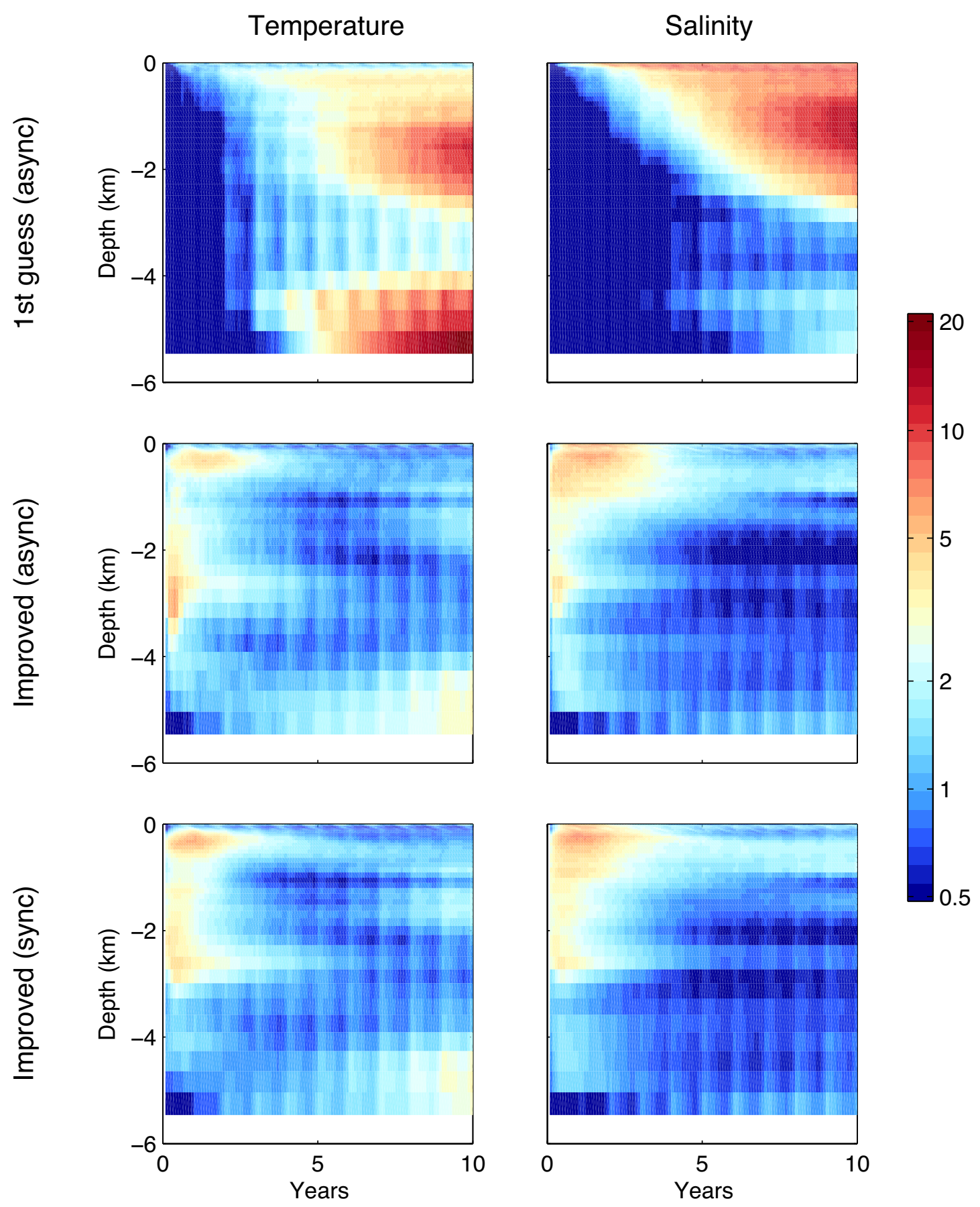

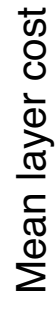

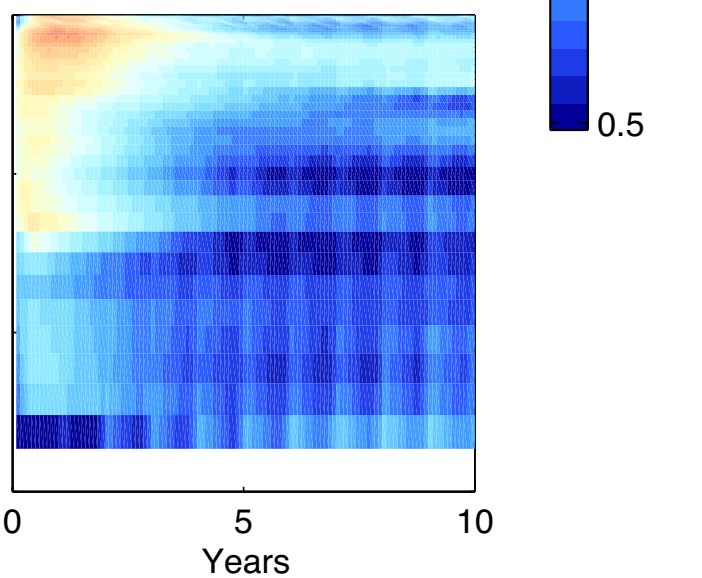

Figure 3-18: Mean layer costs for temperature (left) and salinity (right) in first guess async (top row), improved async (middle row), and improved sync (bottom row). Banded patterns occur because costs are seasonally-varying. Note the nonlinear colorbar. 
Based on the results presented in Figure 3-18, it appears that asynchronous timestepping can be applied to improve the computational efficiency in our state estimation framework. The primary evidence presented is that the resulting inferred controls can be directly used with synchronous timestepping to produce a state estimate that also has a good fit to the target data. It should be noted that this equivalence is likely not universal. In particular, it is possible that model setups that are more highly nonlinear (such as those that are eddypermitting) will not achieve satisfactory results. A similar test should be applied before using asynchronous timestepping in other applications of state estimation.

\subsubsection{Carry-over control}

Carry-over control is an approach developed to address the impact of model drift on the practical application of state estimation. When first guess boundary and initial conditions are used directly in the first iteration of a long state estimate, the model state can drift far from the target data set. As with all nonlinear optimization, the availability of a good first guess that is relatively close to the target data is key to success. Thus, when the model state is allowed to drift very far from the target data, improving the solution through the adjoint method becomes difficult or impossible.

An alternative approach, called carry-over control, works as follows. The key observation is that the estimation procedure is highly effective at reducing cost in shorter estimates and that, in the process, model drift is drastically reduced (see, for example, the top and middle rows of Figure 3-18). Therefore, one starts with a short state estimation period (e.g. 10 years) to obtain an improved estimate of initial and boundary conditions. These adjusted controls are then "carried-over" to a slightly longer estimation period (e.g. 20 years). In the first iteration of the longer estimate, the model will drift from the target data, but the drift is significantly less than that of an experiment without carry-over control. Because the first guess drift is much reduced, the gradients carry more useful information and the estimation procedure is again able to significantly reduce cost. This procedure is repeated for state estimates of increasing length. 

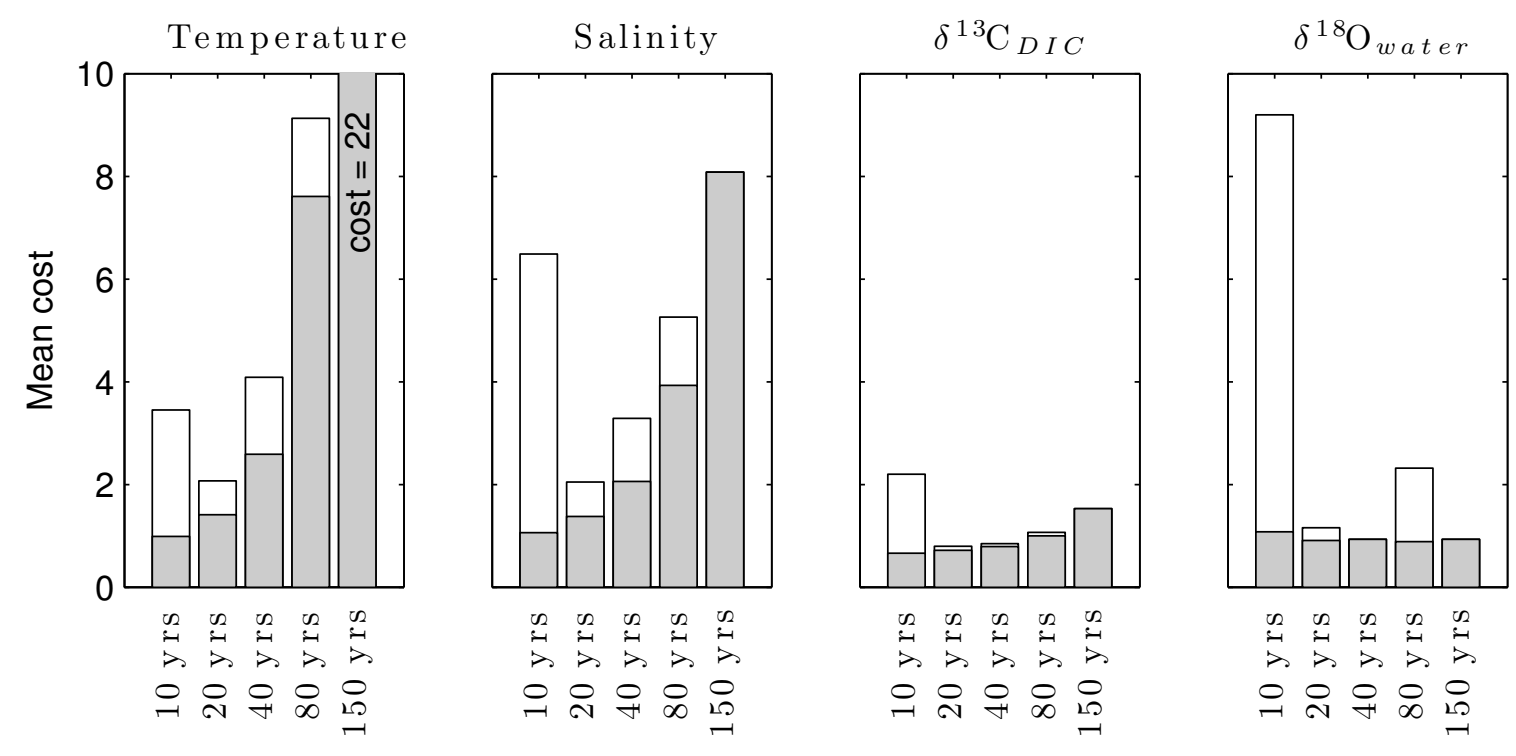

Figure 3-19: An evaluation of mean costs for each data type as run length is increased. The four subfigures show results for each data type, outlined bars shows the first guess solution for each run length, and the solid gray bars show the best solution found for each run length. The first guess solution for the 10 year solution contains no adjustments so the fit is poor. Carry-over control is used for the first guess solution for run lengths 20 - 150, leading to much better solution quality than would otherwise be possible.

To evaluate this approach, carry-over control is applied in a series of state estimates 10, 20, 40, 80, and 150 years in length (Figure 3-19). These experiments use asynchronous timestepping and the model is constrained by OCCA T/S, $\delta^{18} \mathrm{O}_{\text {water }}$, and $\delta^{13} \mathrm{C}_{\text {DIC }}$ data. The 10 year state estimate is initialized from unadjusted first guess controls (black outlined bars at 10 years in Figure 3-19); after 20 iterations, the solution is significantly improved (solid gray bars at 10 yrs in Figure 3-19).

The controls inferred from the 10 year state estimate are carried-over to the first guess estimate for $20 \mathrm{yrs}$, at which point the 20 year solution is optimized to achieve further improvements. This procedure is continued until the 150 year first guess solution is reached. For the configuration used here, the first guess 150 year solution cannot be improved upon because the gradients produced by the adjoint do not carry useful information; the procedure is therefore stopped at 150 years. Costs are not shown for 20 - 150 year estimates 
computed without carry-over controls; these costs would be significantly larger than those of the white bars in Figure 3-19.

Carry-over control successfully enables longer state estimates that have a greatly improved fit to the target data than would be possible without carry-over control. As run length is increased, the fit to $\delta^{13} \mathrm{C}_{\mathrm{DIC}}$ and $\delta^{18} \mathrm{O}_{\text {water }}$ data remains very good (Figure 3-19). However, temperature and salinity costs increase rapidly with run length and become unacceptably large. A detailed analysis of the characteristics of these longer state estimates is provided in the next section; this analysis will show which aspects of carry-over control are effective and what the limitations are.

\subsubsection{Analysis of a longer state estimate: Modern_80yrs}

Since the 150 year solution cannot be improved using the model adjoint, the longest true state estimate in the series is the 80 year estimate. Although the exact time to equilibrium is sensitive to a number of assumptions, the order of magnitude can be understood by considering the results of Wunsch and Heimbach [2008]. These authors estimate that it takes on the order of 500 years for Atlantic waters at a depth of about $2000 \mathrm{~m}$ to come close to equilibrium with a global injection of tracer in the surface ocean, and the time required increases to around 1000 years at about $4000 \mathrm{~m}$. Gebbie and Huybers [2012] use radiocarbon data to estimate ages for the Atlantic Ocean that vary from about 300 to about 800 years at depths below $2000 \mathrm{~m}$. So although 80 years is longer than any modern ocean state estimates we are aware of, this duration is still far too short for the deep ocean to be fully equilibrated with the atmosphere. Keeping this limitation in mind, the characteristics of the 80 year solution, hereafter referred to as "Modern_80yrs", are now examined.

For the isotope data, mean costs for Modern_80yrs are low: 1.0 for $\delta^{13} \mathrm{C}_{\text {DIC }}$ and 0.9 for $\delta^{18} \mathrm{O}_{\text {water }}$. Figure 3-20 shows the same model depth $(2730 \mathrm{~m})$ and GEOSECS data as in Figure 3-17; unlike the 10 year solution, the 80 -year $\delta^{13} \mathrm{C}_{\mathrm{DIC}}$ distribution is physically realistic without obvious evidence of small-scale internal $\delta^{13} \mathrm{C}_{\text {DIC }}$ sources and sinks. CDFs of the normalized misfits of the 80 year solution to the isotopes data are shown in Figure 3- 


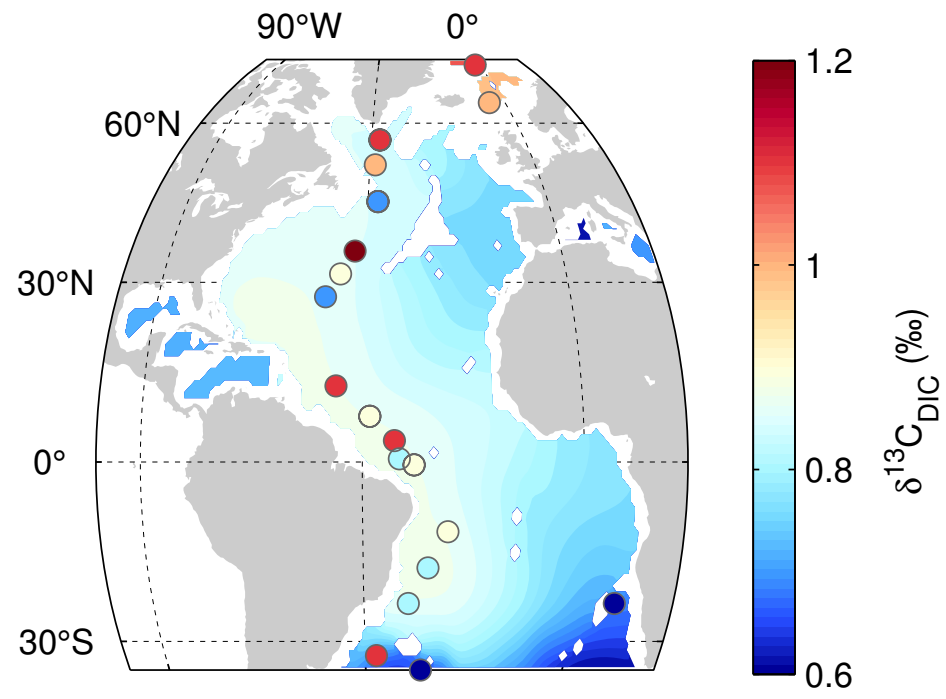

Figure 3-20: The $\delta^{13} \mathrm{C}_{\mathrm{DIC}}$ distribution at $2730 \mathrm{~m}$ for an 80 year modern state estimate.

21. These plots show that $95 \%$ of the modeled $\delta^{18} \mathrm{O}_{\text {water }}$ and $96 \%$ of the modeled $\delta^{13} \mathrm{C}_{\text {DIC }}$ are within $2 \sigma$ of the data - a satisfactory fit. There is some evidence that the model $\delta^{13} \mathrm{C}_{\mathrm{DIC}}$ is biased too low: $70 \%$ of the modeled $\delta^{13} \mathrm{C}_{\text {DIC }}$ values are lower than the GEOSECS values. This bias may be an indication that the remineralization rate assumed here is too low; sensitivity to the assumed rate should be tested in future work.

Table 3.4 shows that the unadjusted (first guess) 10 year solution has a modern-like MOC transport of $15.5 \mathrm{~Sv}$ (see page 114 for a discussion of modern MOC estimates). When the optimization approach is applied to the 10 year estimate to improve the fit of the estimate to the target OCCA properties, the MOC transport decreases to $9.1 \mathrm{~Sv}$; a discussion of individual transport component contributions to this net transport estimate will be provided in the next paragraph. As run length is increased, the estimates with a better fit to the OCCA temperatures and salinities (the "improved estimates") have decreased net MOC transports. That the unadjusted solution has a 15.5 Sv MOC transport implies that the model itself is capable of sustaining a modern-like MOC. Thus, it is the optimization process that is responsible for the reduced MOC transports. Note that the MOC transport in the improved estimates does not decrease over the course of the simulation period; in 

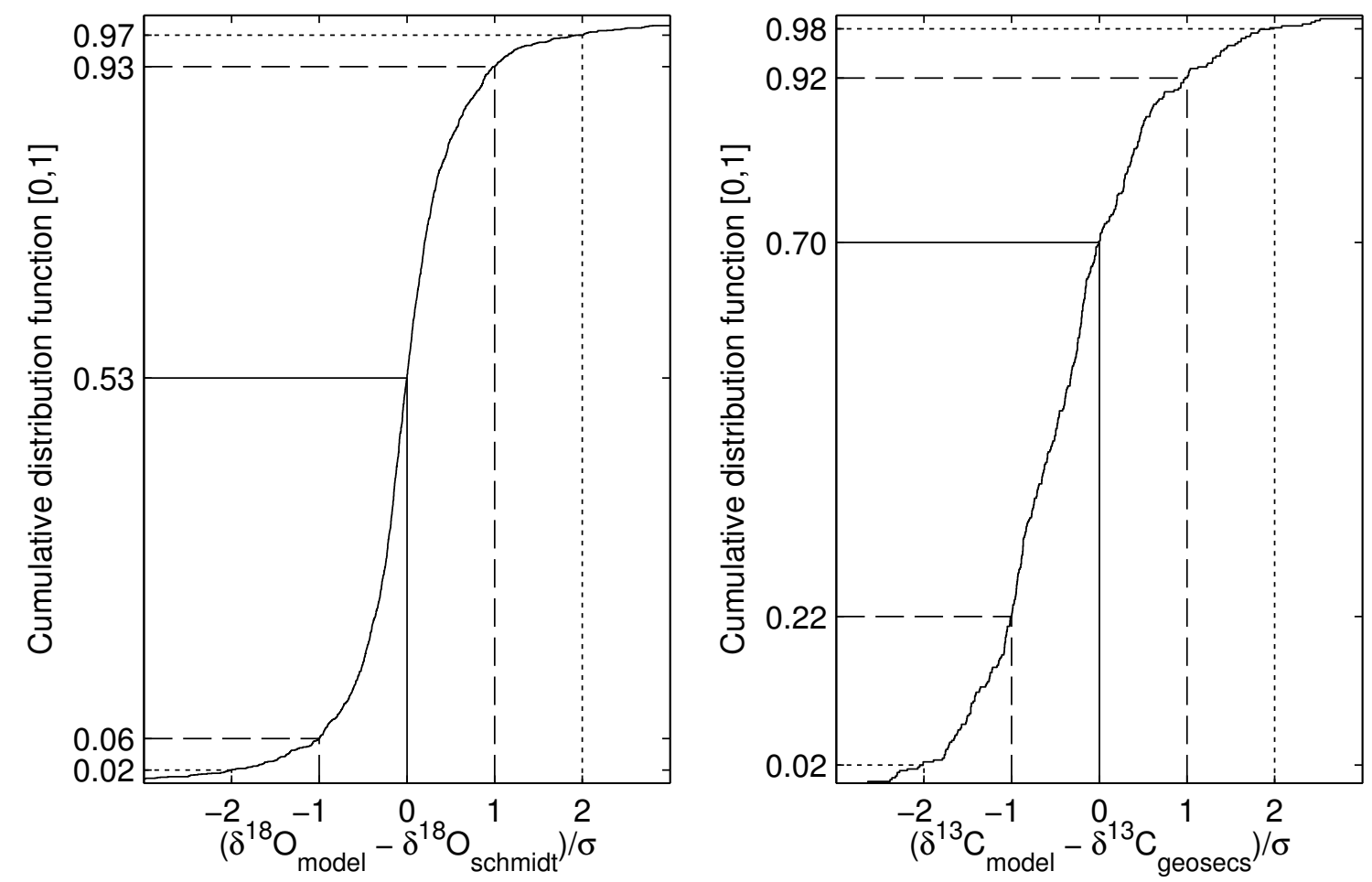

Figure 3-21: CDFs for signed, normalized misfits of the 80 year modern state estimate $\delta^{18} \mathrm{O}_{\text {water }}$ (left) and $\delta^{13} \mathrm{C}_{\text {DIC }}$ (right).

\begin{tabular}{|l|lllll|}
\hline & 10 yrs & 20 yrs & 40 yrs & 80 yrs & 150 yrs \\
\hline First guess & 15.5 & 9.7 & 8.8 & 8.6 & 6.4 \\
Improved estimate & 9.1 & 8.0 & 6.5 & 5.7 & \\
\hline
\end{tabular}

Table 3.4: A comparison of MOC transports in Sv in first guess and improved estimates of 10 to 150 years in length. The MOC transport is defined here as the net transport above $1050 \mathrm{~m}$ at $40^{\circ} \mathrm{N}$; all values are means computed over the second half of the simulation.

fact, each state estimate has MOC transports that are relatively stable in time (not shown). Instead, the MOC transports decrease with iterations of the optimization.

To understand what is happening in the state estimation process, it is useful to compare the 10 year unadjusted, first guess solution against the 10 year improved estimate. As shown in Figure 3-22, the first guess solution has about 1 Sv more transport in both the Gulf Stream and in the surface return flow than OCCA does; since these increases compensate each 
other, the first guess AMOC transport is similar to that of OCCA. In contrast, the improved estimate has a similar Gulf Stream transport as OCCA does, but the improved estimate has a surface return transport that is $23.9 \mathrm{~Sv}$, significantly larger than that of OCCA $(19.3 \mathrm{~Sv})$. The reduced AMOC transport in the improved estimate is therefore due to an increase in the surface return flow, rather than to a reduced Gulf Stream transport.

Figure 3-22 shows a significant shallowing of the MOC in both the first guess and improved estimate solutions as compared to OCCA. There is also notable sloping of streamlines below $1000 \mathrm{~m}$ in the two estimates such that these streamlines are shallower in the north and become deeper towards the low latitudes, while the streamlines for the OCCA estimate tend to be deepest near $35^{\circ} \mathrm{N}$ and to shallow towards the south from that latitude. In a recent review of the modern MOC [Lozier, 2012], a number of different estimates of the AMOC streamfunction are shown in similar latitude-depth plots as shown here; some estimates have relatively flat streamlines, some have streamlines that slope up towards the north, and some have streamlines that slow up towards the south. Apparently the sloping of streamlines in the deep AMOC flow is an aspect of the circulation that is not well constrained.

As shown in Figure 3-23, the fit to OCCA near sea surface temperature and salinity is greatly improved between the first guess and improved estimates (near surface is defined here to be the top most 10 meters of the model). The normalized misfits for the improved estimate (Figure 3-23, bottom row) are acceptable in most regions; an exception is evident in the temperatures of the eastern equatorial region, which remain too high. OCCA temperatures in this region were difficult to reproduce in the Modern_TS estimate as well (see Figure 3-5). Figure 3-24 shows the same analysis as in Figure 3-23 but at a depth of $1100 \mathrm{~m}$. The most notable characteristic of the first guess solution is that the waters north of $45^{\circ} \mathrm{N}$ are too warm and salty. In the adjusted solution, the western half of this region remains too warm and most of the region north of $45^{\circ} \mathrm{N}$ has become too fresh instead of too salty. The next section discusses a number of potential explanations for these observations. 
OCCA -- MOC and transports at $29^{\circ} \mathrm{N}$
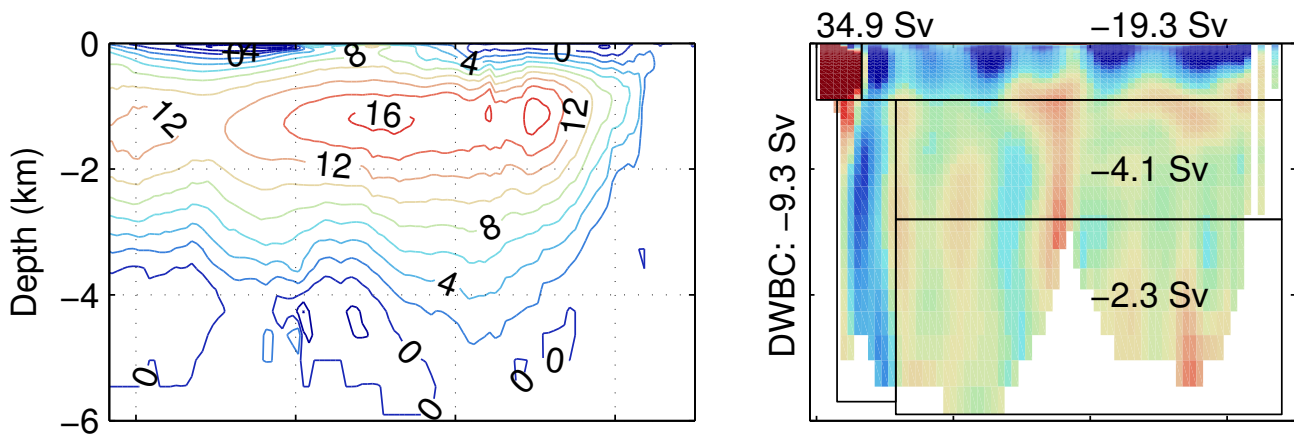

1st guess estimate $--\mathrm{MOC}$ and transports at $29^{\circ} \mathrm{N}$
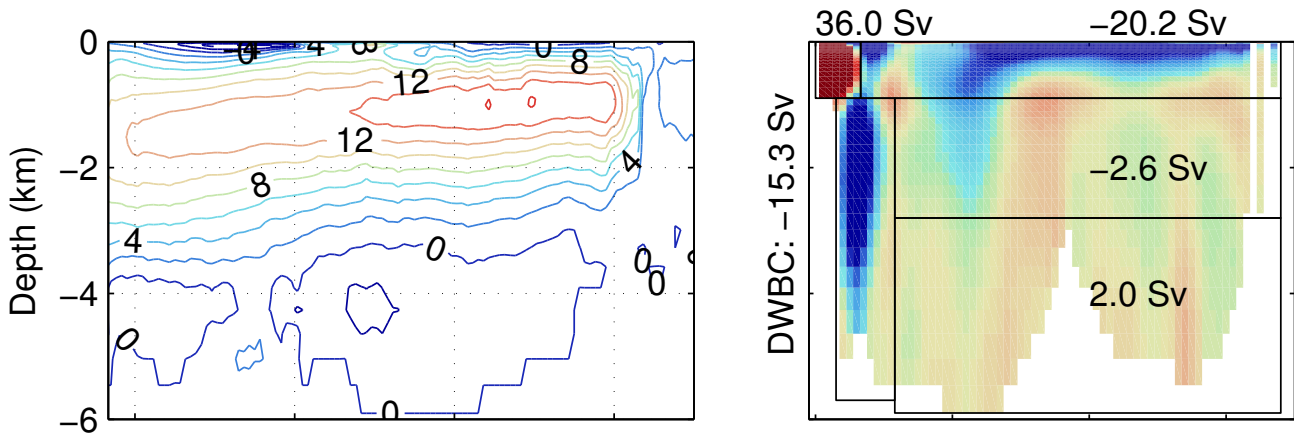

Improved estimate -- MOC and transports at $29^{\circ} \mathrm{N}$
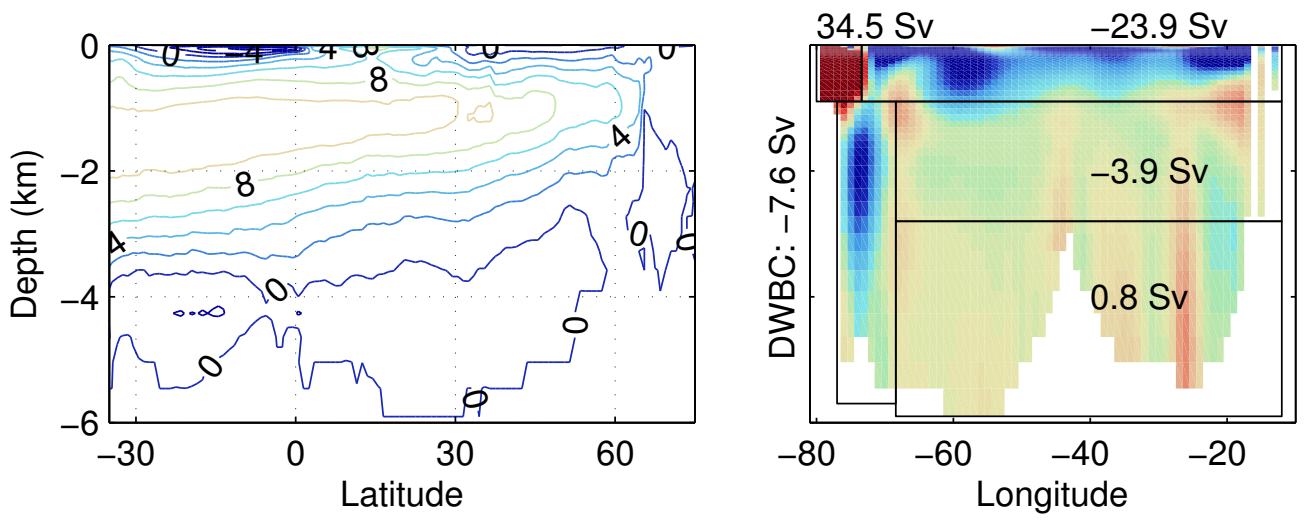

Figure 3-22: The MOC streamfunction in Sv (left) and meridional velocities in $\mathrm{cm} / \mathrm{s}$ (right) for OCCA (top row), the first guess 10 year state estimate (middle row), and the improved 10 year state estimate (bottom row). Although the improved estimate has a better fit OCCA temperatures and salinities overall, the MOC is a worse representation of the modern Atlantic MOC. 
Temperature
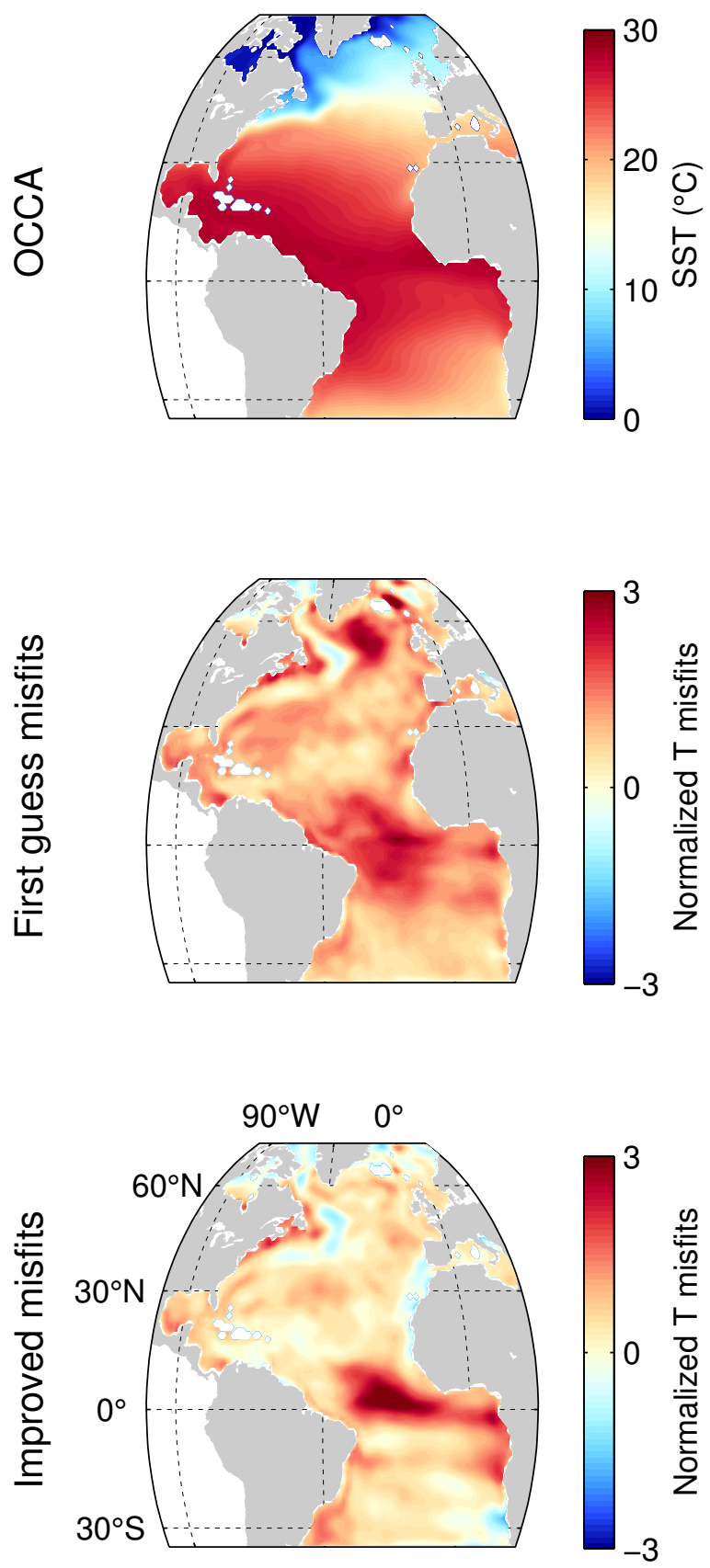

Salinity
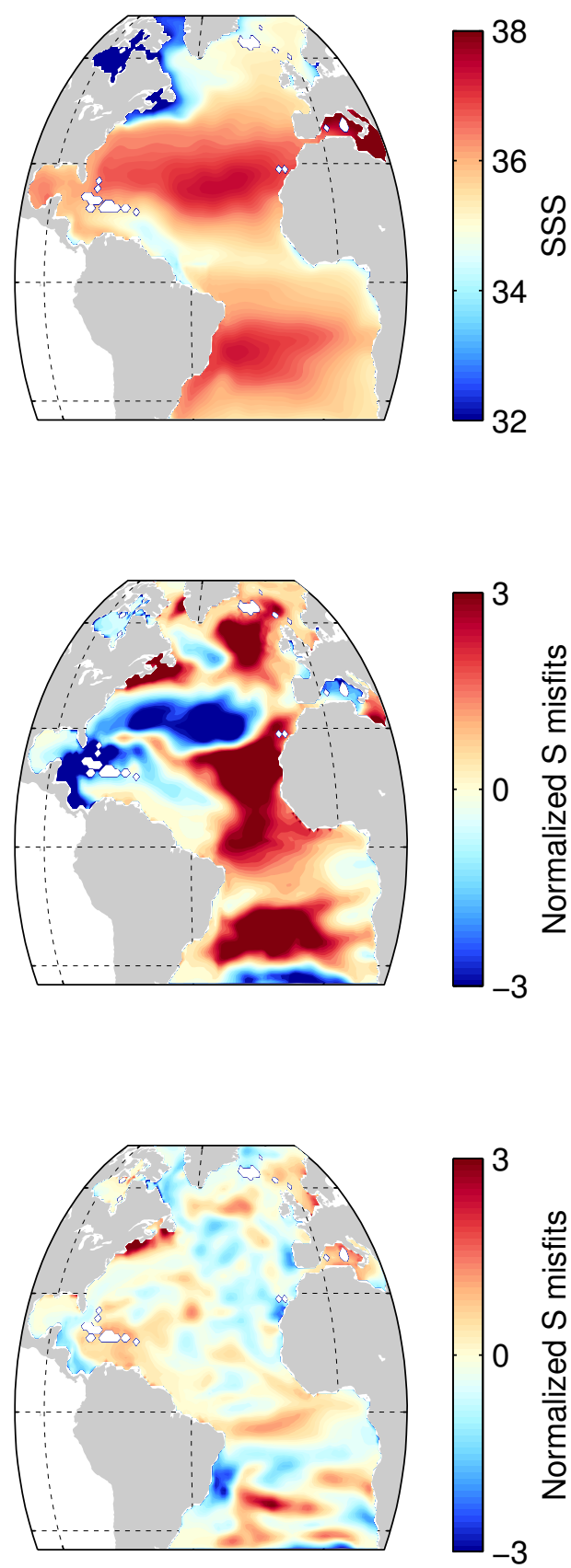

Figure 3-23: Annual mean near sea surface temperature and salinity in OCCA (top row) and normalized misfits (model - occa/ $\sigma$ ) for the same fields in first guess async (middle row) and improved async (bottom row) estimates. 
Temperature
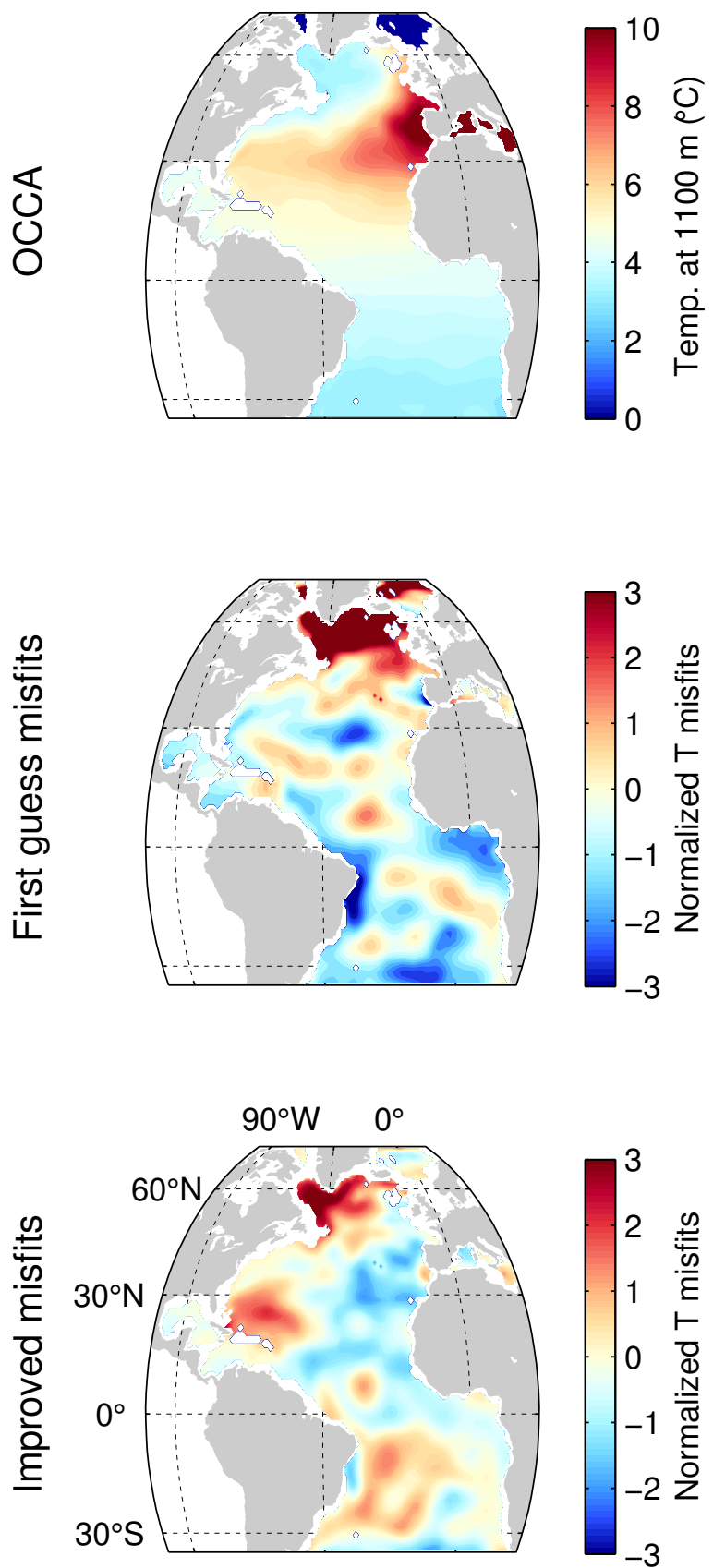

Salinity
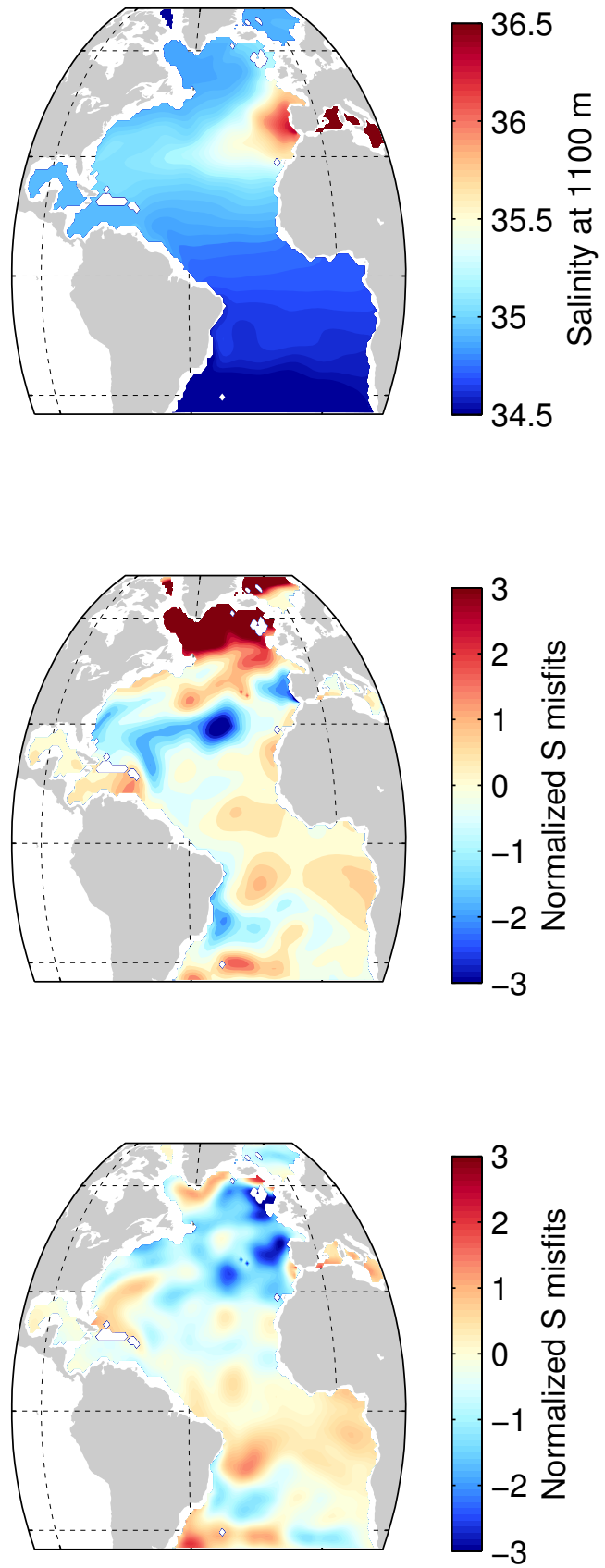

Figure 3-24: Annual mean temperature and salinity at $1100 \mathrm{~m}$ in OCCA (top row) and normalized misfits (model - occa/ $\sigma$ ) for the same fields in the first guess (middle row) and improved (bottom row) estimates. 


\subsubsection{Current limitations in equilibrium ocean estimation}

Analysis of the Modern_TS estimate (Section 3.3) showed that the state estimate configuration used in this thesis is sufficient for reproducing OCCA temperature and salinity conditions in a two year state estimate. OCCA is an average of three one year state estimates of modern ocean conditions from 2004, 2005, and 2006. It is reassuring, though not particularly surprising, that these conditions can be reproduced here. Section 3.5.3 showed that the same statement can not be made when the estimate length is extended to 10 years or longer; it has not been possible here to obtain ocean state estimates that have (1) a close fit to OCCA T/S, (2) have an AMOC that is similar to today's, and (3) that are 10 years in length or longer. It appears relatively straightforward to obtain two of these three characteristics: Modern_TS has properties (1) and (2), the first guess estimate described on page 137 has properties (2) and (3), and the improved estimate (also described on page 137) has properties (1) and (3). The specific source of the difficulty in achieving properties (1)-(3) in the same estimate is unknown. A number of possible explanations are discussed below.

OCCA is an inappropriate target. The OCCA representation of the modern ocean may be an inappropriate target for such an effort. Deep ocean observations remain sparse, and OCCA is based on only three years of observations (in addition to climatology). Thus, OCCA's deep ocean properties are underconstrained and may not be an adequate representation of the modern ocean for current purposes. Longer estimates of modern ocean state that include more years of observations could be used to explore this idea (e.g., ECCO Version 3 includes 17 years of observations, Wunsch et al. 2009).

Others types of temporal variability must be incorporated. The model configurations used here lack many types of temporal variability. Atmospheric control adjustments are restricted to monthly means, under the assumption that long term mean atmospheric conditions are key controls on the large scale properties of the ocean. It is possible that higher frequency variability is pivotal (i.e. the diurnal cycle, or high intensity, short timescale storm events). It is also possible that interannual or longer period variability is key to setting mean ocean properties. 
The model is inadequate. The test we put to the model (to produce a long running state estimate with stable properties that are close to a given target) is a demanding one. It is possible that the parameterizations used here for horizontal and vertical mixing are insufficient representations of these processes for this test. A case in point is provided by recent development on ECCO Version 4: the model exhibits decreases in AMOC strength over the simulation period unless the model is augmented with an additional parameterization for the effect of salt plumes in sea ice formation regions (P. Heimbach, pers. comm.); the original parameterizations for vertical convection were inadequate. It is possible that model configuration inadequacies are also responsible for the decreased AMOC strength seen here. Other model configurations could be tested, or model parameters such as the diapycnal diffusivity could be used as control variables.

The steady state assumption can not be applied to the modern ocean. Although some form of the steady state assumption is employed relatively often in oceanography (e.g., consider the popularity of climatology for describing modern climate), the extent to which the modern ocean is consistent with a steady state assumption is unknown. It is possible that the modern ocean state is quite far from equilibrium with the modern atmosphere. For example, if atmospheric properties have changed significantly over the timescales of deep ocean ventilation, it would be impossible to find a single, long running ocean state estimate in which surface and deep ocean properties are both close to modern conditions. An evaluation of the extent to which the modern ocean is consistent with some sort of steady state assumption would be of great value, both to the work in this thesis and to many other areas of oceanography. The following test may prove useful to this evatluation: a very strong penalty on model drift could be added to the state estimate configuration used in this chapter. Such a constraint may permit identification of an ocean state estimate that is close to steady state, and yet not as far from modern ocean conditions as forward model simulations often are. If such a solution could be identified, the differences between the model state and the modern ocean could provide clues as to the ways in which the modern ocean is out of equilibrium with the atmosphere. 


\subsection{Chapter summary}

This chapter has described the ocean model and state estimation techniques used in this thesis. These techniques have been tested using modern temperature, salinity, $\delta^{18} \mathrm{O}_{\text {water }}$, and $\delta^{13} \mathrm{C}_{\text {DIC }}$ data in state estimates from 2 - 150 years long. The Modern_TS solution, a two year state estimate, was shown to be consistent with OCCA and with independent estimates of modern ocean transports. The basic functionality of the isotope modeling approach was demonstrated through the 2-year Modern_Iso solution, though this estimate also revealed the inadequacy of short state estimates for reconstructing deep ocean conditions using sparse data. A series of state estimates 10 - 150 years in length showed that longer state estimates are possible, and are advantageous for inversion of sparse deep ocean tracer data. Several new approaches were described: (1) the inclusion of a simple approach to modeling $\delta^{18} \mathrm{O}_{\text {water }}$ and $\delta^{13} \mathrm{C}_{\text {DIC }}$ in the MIT GCM, and (2) the application of asynchronous timestepping and carry-over control to permit longer state estimates.

The state estimation configurations employed here were specifically designed with the goal of identifying a seasonal steady state estimate (i.e. an equilibrium ocean estimate or EOE). This work has identified significant challenges to the goal of EOE: it has not been possible to build a state estimate that simultaneously satisfies all three desired characteristics: long-running, close to the OCCA T/S estimate, and with a modern-like MOC. Issues that may have contributed to the difficulty in obtaining an EOE were identified in Section 3.5.4, and possible avenues for future investigation were discussed. Although it has not been possible to achieve full equilibrium ocean estimates, the estimation approach described in this chapter appears to have significant skill in reproducing many aspects of the modern ocean state. In the next two chapters, this state estimation approach is applied to build estimates of the Last Glacial Maximum ocean state. In interpreting the results of those chapters, the reader is encouraged to use this chapter as a reference for the skills and limitations of the estimation approach. 


\section{Chapter 4}

\section{Upper ocean conditions at the Last Glacial Maximum}

In this chapter, the state estimation approach described in Chapter 3 is used to build estimates of LGM upper ocean conditions that are constrained by proxy records. We do not claim to know the actual LGM ocean state - rather, the goal is to build an estimate that is consistent with the available data and with known ocean dynamics. Such an estimate is likely to be a good representation of some aspects of the LGM ocean and a poor representation of others. Comparisons with included and withheld data, and with the results of other LGM models, help to identify strengths and weaknesses of the estimate.

Many assumptions are required to proceed with this effort. In a hypothetical (and nonexistent) global ocean model with perfectly prescribed sea surface conditions and perfect physics, the full three-dimensional flow found by running the model out to equilibrium would be a good representation of the real ocean state in question (assuming the real ocean is well-represented by an equilibrium or steady state solution). In practice, LGM sea surface conditions are imperfectly known, the model physics are imperfect, running to equilibrium is not possible, and the extent to which the LGM ocean was in equilibrium is unknown. Thus, important assumptions must be made. These are as follows.

- It is assumed that the forcing and response of the upper ocean at the LGM can be 
reasonably approximated by a repeated seasonal cycle.

- The model representation of Ekman pumping, buoyancy forcing, and mixed layer dynamics is assumed to be appropriate. This assumption is supported by the many previous applications of the MIT GCM (see Section 3.1). The upper ocean state estimates in this chapter are therefore assumed to have some skill through the mixed layer and the thermocline, though the uncertainty of the estimates must be assumed to increase with depth.

- To permit exploration of a variety of state estimate configurations, state estimates 10 years in length are used. It is assumed that this estimate length is sufficiently long that upper ocean properties are largely defined by atmospheric forcing, rather than by initial conditions.

- Amongst satisfactory solutions, solutions that are close to the modern climate are preferred over solutions that are wildly different. In the absence of evidence from data or theory requiring large LGM changes, they should be avoided.

- The state estimates in this chapter are constrained by the MARGO proxy reconstructions of LGM near sea surface temperatures (Waelbroeck et al. [2009], see Section 2.2.2 for background and full references). Other NSST proxy records exist, and their incorporation would provide improved geographic coverage, among other benefits. However, significant effort would be required for each additional record added; for example, considerations include proper age control, identification of any interlaboratory calibration issues, and assignment of uncertainty estimates in a manner consistent with the MARGO compilations. These are issues best evaluated by experts in the specific proxy type under consideration.

Chapter 3 described the general structure of the forward model (Section 3.1) and the state estimation approach (Section 3.2). Adaptations of the forward model (Section 4.1) and the state estimation approach (Section 4.2) for the study of the LGM upper ocean 

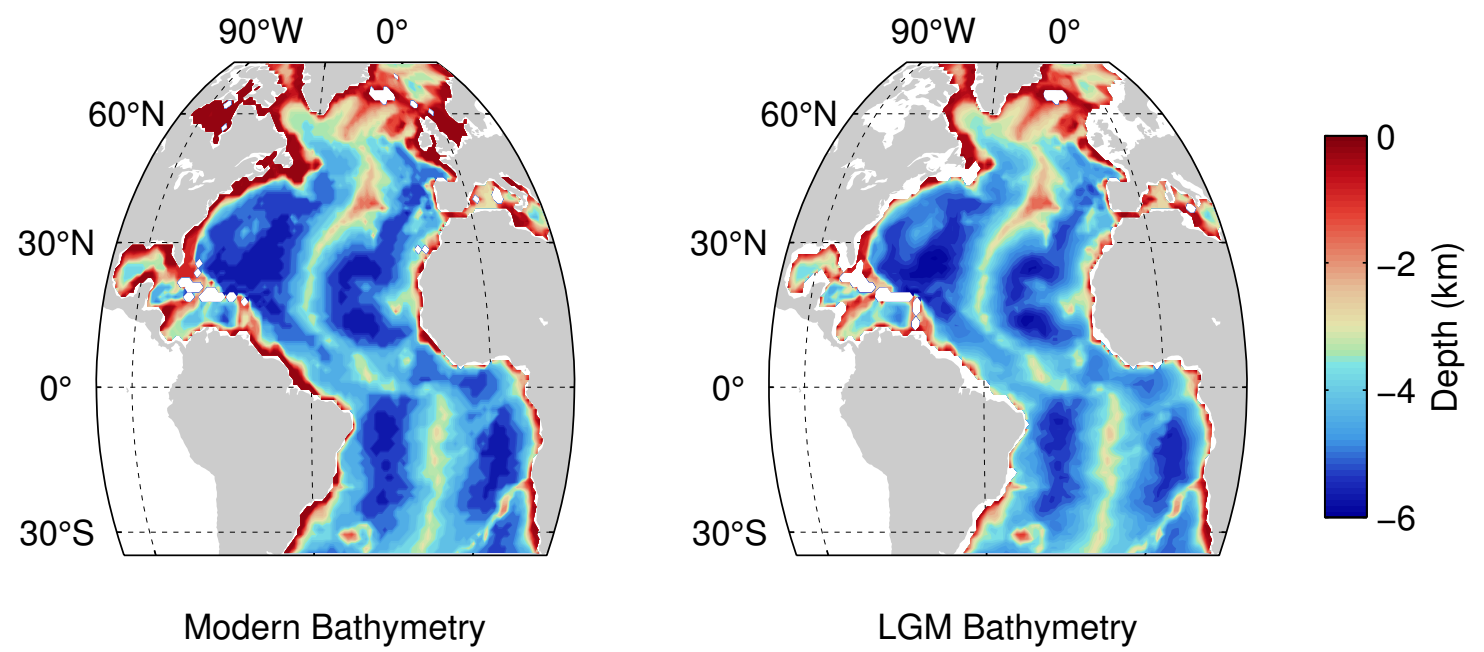

Figure 4-1: The modern bathymetry, adapted from OCCA (left), and the LGM bathymetry, adapted from ICE-5G (right, data from Peltier [2004]). Dry regions in each bathymetry are white; the modern continents are shaded in gray for reference.

are described here. A variety of different state estimates are presented in Section 4.3 to explore assumptions of the state estimation setup. These explorations are used to motivate assumptions used for our best estimate solution, called LGM_Upper, which is is described and analyzed in Section 4.4. The results are compared to previous work in Section 4.5 and Section 4.6 concludes the chapter.

\subsection{Forward model}

The LGM forward model follows most aspects of the modern forward model (see Section 3.1); modifications for the LGM case include the bathymetry, initial conditions, and southern boundary conditions. An interpolated, smoothed version of the ICE-5G [Peltier, 2004] bathymetry is used (Figure 4-1). Key differences with the modern bathymetry include closure of Hudson Bay, a reduction in depth of the Straits of Gibraltar and the Florida Straits, and exposure of the shallow continental shelves along eastern North America and off the coast of Europe. 


\subsubsection{First guess initial conditions}

A first guess must be made for initial conditions of temperature and salinity; note that these are simply first guess estimates and that adjustments to these estimates will be permitted. Since all state estimates presented in this chapter are 10 years in length, upper ocean conditions are significantly affected by atmospheric forcing, which will also be adjusted as part of the optimization process. The choice of first guess initial conditions is thus most important for deep ocean conditions, and it is on these that we focus. One practical issue must be considered: the state estimation approach used in this thesis works more effectively when starting from more strongly stratified ocean conditions. When starting from first guess conditions that are weakly stratified, convective events are common; these ocean mixing processes are strongly nonlinear, and complicate use of the adjoint model to improve the solution.

Consider first the choice of an initial temperature distribution. As discussed in Section 2.2.3, evidence from pore fluid records indicates that the LGM deep ocean may have been significantly colder than today. Differences between MARGO NSST records and modern temperatures in the high latitude North Atlantic indicate that LGM NSSTs may also have been significantly colder than today (see Figure 2-3); assuming some deep Atlantic waters formed in the North Atlantic at the LGM, colder NSSTs are an indication that deep waters may have been significantly colder as well. The modern deep Atlantic Ocean is marked by temperatures that vary from near $0.4^{\circ} \mathrm{C}$ in regions with strong $\mathrm{AABW}$ influence to temperatures near $3^{\circ} \mathrm{C}$ around $2000 \mathrm{~m}$ in the subpolar gyre ${ }^{1}$. The minimum possible temperature is constrained by the freezing point of seawater, which is $-2^{\circ} \mathrm{C}$ for a canonical modern ocean salinity of 35 , but increases at lower salinities. Based on these considerations, the initial temperature distribution is given by OCCA temperatures made $3^{\circ} \mathrm{C}$ colder everywhere; since this distribution results in temperatures below the freezing point in many subsurface locations (an unphysical situation), all temperatures below $-1.7^{\circ} \mathrm{C}$ are set to $-1.7^{\circ} \mathrm{C}$. The minimum is set to $-1.7^{\circ} \mathrm{C}$ to avoid below-freezing temperatures for

\footnotetext{
${ }^{1}$ See, e.g., http://www.ewoce.org/gallery/A16_TPOT.gif.
} 
fresher-than-average ocean waters.

Next, consider the choice of an initial salinity distribution. Due to freshwater storage in land-based ice sheets, the LGM mean ocean salinity was higher by about 1.1 [Adkins and Schrag, 2001]. Evidence from pore fluid records indicates that the LGM Atlantic Ocean may also have been marked by southern source waters that were particularly salty as compared to northern source waters (see Section 2.2.3). If deep ocean waters were generally significantly colder, deep ocean thermal stratification would likely have been weaker (due to the minimum set by the freezing point of seawater); that the haline stratification was stronger than today's at the LGM seems a promising hypothesis. An initial salinity field is thus generated from OCCA salinities by increasing both the total salt content and the haline stratification as follows. Salinities below $4000 \mathrm{~m}$ are increased by 2.75 , salinities between 4000 and $3000 \mathrm{~m}$ are increased by 2.06, salinities between 3000 and $2000 \mathrm{~m}$ are increased by 1.38 , salinities between 2000 and $1000 \mathrm{~m}$ are increased by 0.69 , and salinities between $1000 \mathrm{~m}$ and the surface are not modified. These adjustments result in a mean salinity change over the model domain of 1.1 .

Sensitivity to the choice of first guess initial conditions has not been considered in this thesis, but should be investigated in future work. In particular, the choice to modify the modern salinity field in a stepped fashion by depth level was made for reasons of convenience; an alternative choice that should be tested is to modify the modern salinity field by adding a linear profile of salinity with depth (where the profile was defined to still modify the mean salinity by 1.1 ).

\subsubsection{First guess atmospheric forcing}

As was the case for initial conditions, selection of a first guess for atmospheric forcing is a challenge: the characteristics of the LGM atmosphere are not well constrained. Ideally, the choice of a first guess atmospheric forcing would be largely irrelevant - one could start from a variety of first guesses and obtain the same inferred LGM atmospheric conditions. In practice, optimization generally benefits from the availability of a decent first guess. 
Forward model estimates of LGM atmospheric conditions vary widely (see Section 2.3.1), and initial attempts to use the results of a coupled model simulation to drive our model were unsuccessful. It is not expected that the LGM atmosphere was drastically different from today's - available data are more indicative of relatively modest changes (see Section 2.1). Given the absence of a better first guess, the modern atmospheric forcing used in Chapter 3 (NCEP 2006) is used here. The sensitivity of the solution to the choice of a first guess atmospheric forcing should be explored in future work.

\subsection{Adjoint model}

The state estimation configuration follows the modern setup (Section 3.2) with a few exceptions. A key difference is in the assignment of uncertainty estimates for control variables - the LGM initial and boundary conditions are clearly more poorly known than those of the modern ocean. However, choosing large uncertainties can easily lead to ocean states that are wildly different than the modern ocean (e.g. with Gulf Stream transports twice the modern). As laid out in the introduction to this chapter, the philosophy taken here is that estimates that are closer to the modern circulation are preferred, unless there is proxy evidence that requires large LGM changes. As a conservative initial estimate, uncertainties on atmospheric forcing for the LGM are taken to be twice the modern uncertainties; these are listed under the column labeled LGM_AllSSTs in Table 4.1. A number of different assumptions are tested later in the chapter; these uncertainties are listed in other columns of the table for later reference.

Southern boundary condition uncertainties on temperature and salinity are taken to be $1^{\circ} \mathrm{C}$ and 0.2 at all depths; the same ratio of baroclinic to barotropic mode uncertainty is used as for the modern. The modern T/S uncertainties of Forget and Wunsch [2007] are used as initial condition uncertainties. Although larger T/S changes are expected for the LGM case, this choice was made for practical reasons - with uniform and/or larger uncertainties, the optimization is not able to achieve sufficient solution improvement; this behavior may 
be related to ill-conditioning of gradients or inadequate data. In any case, upper ocean behavior, the topic of this chapter, is dominated by atmospheric forcing after short integration times. Therefore, the choice of initial conditions and initial condition adjustments is not expected to be of primary importance here.

The cost function is defined as follows. The $J_{c t r l}$ cost contribution is that of Modern_TS (Section 3.2.3), but with the LGM-specific uncertainties defined above. The model-data misfit contribution is

$$
\begin{aligned}
& J_{M A R G O}=\frac{2}{n y} \sum_{y=n y / 2}^{n y}[ \\
& \frac{1}{3}\left(\overline{\mathbf{N S S T}_{\text {ann }}}-\mathbf{N S S T}_{\text {annFor }}\right)^{T} \mathbf{W}_{\text {annFor }}\left(\overline{\mathbf{N S S T}_{\text {ann }}}-\mathbf{N S S T}_{\text {annFor }}\right) \\
& +\frac{1}{3}\left(\overline{\mathbf{N S S T}_{\text {win }}}-\mathbf{N S S T}_{\text {winFor }}\right)^{T} \mathbf{W}_{\text {winFor }}\left(\overline{\mathbf{N S S T}_{\text {win }}}-\mathbf{N S S T}_{\text {winFor }}\right) \\
& +\frac{1}{3}\left(\overline{\mathbf{N S S T}_{\text {sum }}}-\mathbf{N S S T}_{\text {sumfor }}\right)^{T} \mathbf{W}_{\text {sumfor }}\left(\overline{\mathbf{N S S T}_{\text {sum }}}-\mathbf{N S S T}_{\text {sumFor }}\right) \\
& +\left(\overline{\mathbf{N S S T}_{\text {ann }}}-\mathbf{N S S T}_{\text {annAlk }}\right)^{T} \mathbf{W}_{\text {annAlk }}\left(\overline{\text { NSST }_{\text {ann }}}-\text { NSST }_{\text {annAlk }}\right) \\
& +\left(\overline{\mathbf{N S S T}_{\text {ann }}}-\mathbf{N S S T}_{\text {annMgCa }}\right)^{T} \mathbf{W}_{\text {annMgCa }}\left(\overline{\mathbf{N S S T}_{\text {ann }}}-\mathbf{N S S T}_{\text {annMgCa }}\right) \\
& +\left(\overline{\mathbf{N S S T}_{\text {sum }}}-\mathbf{N S S T}_{\text {sumMgCa }^{T}}\right)^{T} \mathbf{W}_{\text {sumMgCa }}\left(\overline{\mathbf{N S S T}_{\text {sum }}}-\mathbf{N S S T}_{\text {sumMgCa }_{\text {a }}}\right) \\
& +\frac{1}{3}\left(\overline{\mathbf{N S S T}_{\text {ann }}}-\mathbf{N S S T}_{\text {annDino }}\right)^{T} \mathbf{W}_{\text {annDino }}\left(\overline{\text { NSST }_{\text {ann }}}-\text { NSST }_{\text {annDino }}\right) \\
& +\frac{1}{3}\left(\overline{\mathbf{N S S T}_{\text {win }}}-\mathbf{N S S T}_{\text {winDino }}\right)^{T} \mathbf{W}_{\text {winDino }}\left(\overline{\mathbf{N S S T}_{\text {win }}}-\mathbf{N S S T}_{\text {winDino }}\right) \\
& \left.+\frac{1}{3}\left(\overline{\mathbf{N S S T}_{\text {sum }}}-\mathbf{N S S T}_{\text {sumDino }}\right)^{T} \mathbf{W}_{\text {sumDino }}\left(\overline{\mathbf{N S S T}_{\text {sum }}}-\mathbf{N S S T}_{\text {sumDino }}\right)\right]
\end{aligned}
$$

where the sum is over the second half of the model run and costs are summed for annual, winter, and summer seasons for foraminifera (a-c), alkenones (d, annual mean only), $\mathrm{Mg} / \mathrm{Ca}$ (e-f, annual mean and summer only), and dinoflagellate cysts (g-i). $\overline{\mathbf{N S S T}_{\mathbf{a n n}}}$, $\overline{\mathbf{N S S T}_{\text {win }}}$, and $\overline{\mathbf{N S S T}_{\text {sum }}}$ are the annual mean, January-March, and July-September means of the modeled temperatures over depths of 0-30 m. The weighting matrices are given by the squared inverses of $\sigma_{M A R G O}$, the MARGO defined uncertainties (see Appendix A for full 


\begin{tabular}{|l|l|l|l|l|}
\hline Variable & Units & LGM_AllSSTs & LGM_ForamsX2 & LGM_Upper \\
\hline Shortwave radiation & $\mathrm{W} / \mathrm{m}^{2}$ & 20 & 40 & 20 \\
2-m air temperature & ${ }^{\circ} \mathrm{C}$ & 2 & 4 & 4 \\
10-m zonal wind speed & $\mathrm{m} / \mathrm{s}$ & 2 & 4 & 2 \\
10-m merid wind speed & $\mathrm{m} / \mathrm{s}$ & 2 & 4 & 2 \\
2-m Specific humidity & $\mathrm{kg} / \mathrm{kg}$ & $2 \cdot 10^{-3}$ & $4 \cdot 10^{-3}$ & $2 \cdot 10^{-3}$ \\
Precipitation & $\mathrm{m} / \mathrm{s}$ & $4 \cdot 10^{-8}$ & $8 \cdot 10^{-8}$ & $4 \cdot 10^{-8}$ \\
\hline
\end{tabular}

Table 4.1: Prior uncertainties for atmospheric controls in three different LGM state estimate configurations.

details on these uncertainty estimates). To ensure equal weight is given to each independent measurement, a factor of $1 / 3$ is applied to the foraminifera and dinocyst assemblagebased records. For these proxies, a single sample is used to reconstruct annual, winter, and summer means; although all three seasons are used in the cost function, they are not independent.

Note that all analyses presented in this chapter describe model conditions in year 8 of the 10 year estimates, unless otherwise noted. This year is close to the midpoint of the period included in the cost function (years 6 - 10, inclusive); as will be shown, the state estimation approach applied here is effective at reducing model drift for this cost function period, so that it is expected that the results of the analyses would be similar if a different year were analyzed (or indeed, if an average of the last 5 years were analyzed).

\subsection{Exploration of assumptions}

A number of state estimates are examined in this section to explore the assumptions of the state estimation setup. In Chapter 3, cumulative distribution functions (CDF), cost analyses, and misfits were used to analyze experiments. Here, it is practical to evaluate the fit of the model to the data overall, but also to evaluate the fit to each data subset individually. The following diagnostics have been identified as particularly useful for these purposes. The mean cost is computed over a given subset of data: mean $\left.[(\operatorname{model}-d a t a) / \sigma)^{2}\right]$; a value 
of order one is desirable. The fraction within $2 \sigma$ is the fraction of modeled values that fall within $\pm 2 \sigma$ of the data (as can be read from a CDF plot); a value $\geq 0.95$ is desirable. The fraction below 0 is the fraction of model values that are colder than the corresponding data values (as can be read from a CDF plot); a value below 0.5 indicates the model is too warm relative to the data while a value near 0.5 indicates bias is not a significant problem.

\subsubsection{Using all MARGO data as published: LGM_AllSSTs}

A state estimate is computed using all MARGO data and uncertainties as published. After 30 iterations, cost is reduced by $68 \%$ from the first guess solution; cost did not decrease with additional iterations. The iteration 30 solution will hereafter be referred to as "LGM_AllSSTs". Figure 4-2 shows that only 86\% of the LGM_AllSSTs model values fall within $2 \sigma$ of the data and that $34 \%$ of modeled values are too cold relative to the data. In CDFs for annual, winter, and summer mean misfits alone (not shown), 33\%, 38\%, and 31\%

of the modeled values are too cold, respectively. Thus, LGM_AllSSTs is too warm relative to the data in general, and the bias is stronger in summer than in winter.

As shown in Figure 4-3, the LGM_AllSSTs solution is an improvement over the first guess solution on nearly all metrics. However, the improved solution is still unacceptable as significantly less than $95 \%$ of the modeled values fall within $2 \sigma$ of the data. There is also bias in the model-data misfits: the model is too cold relative to a majority of the dinoflagellate cyst data but is too warm relative to a majority of the foram assemblage and $\mathrm{Mg} / \mathrm{Ca}$ data. The datasets are inconsistent with each other and the state estimate is a compromise. That LGM_AllSSTs is statistically unacceptable implies that one or more assumptions made in the state estimation framework is incorrect. Key possibilities include the following.

1. Given the results seen for the LGM_AllSSTs estimate, it is likely that data uncertainties are not large enough. MARGO uncertainties are published as semi-quantitative and known only within a constant multiplier (for LGM_AllSSTs a multiplier of one was used). These uncertainties consider calibration error, dating uncertainty, the 


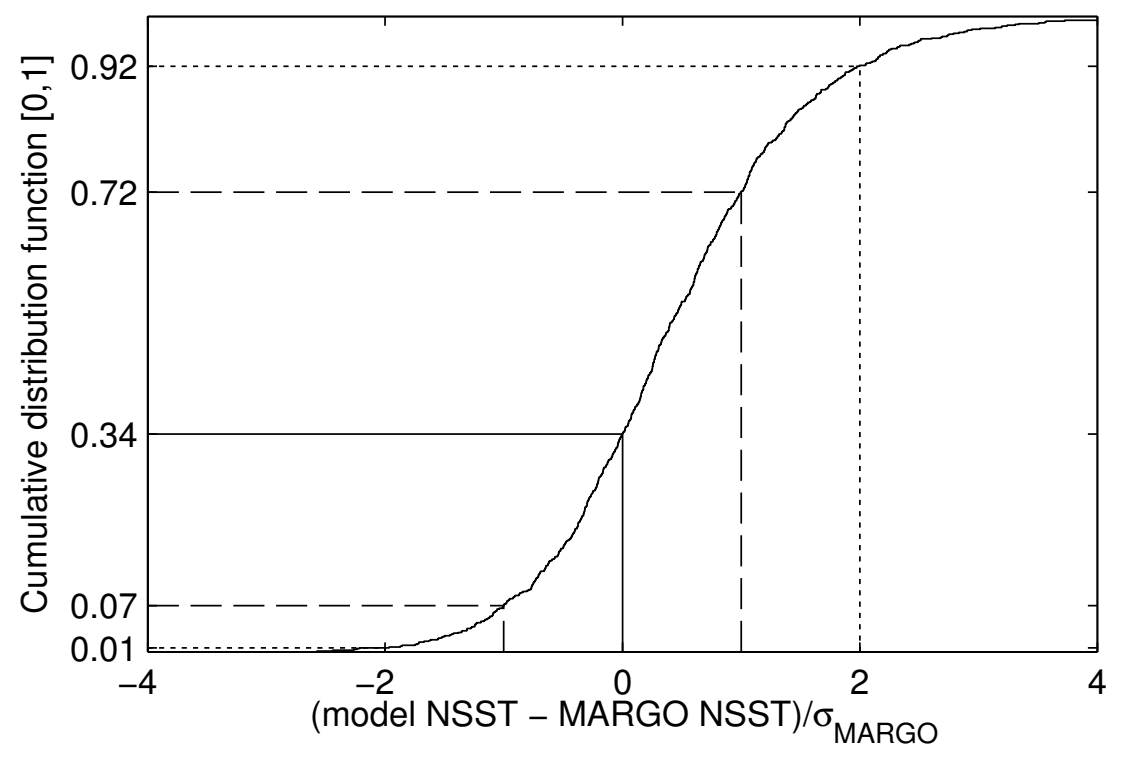

Figure 4-2: CDF of the normalized misfit of NSSTs in LGM_AllSSTs to MARGO.
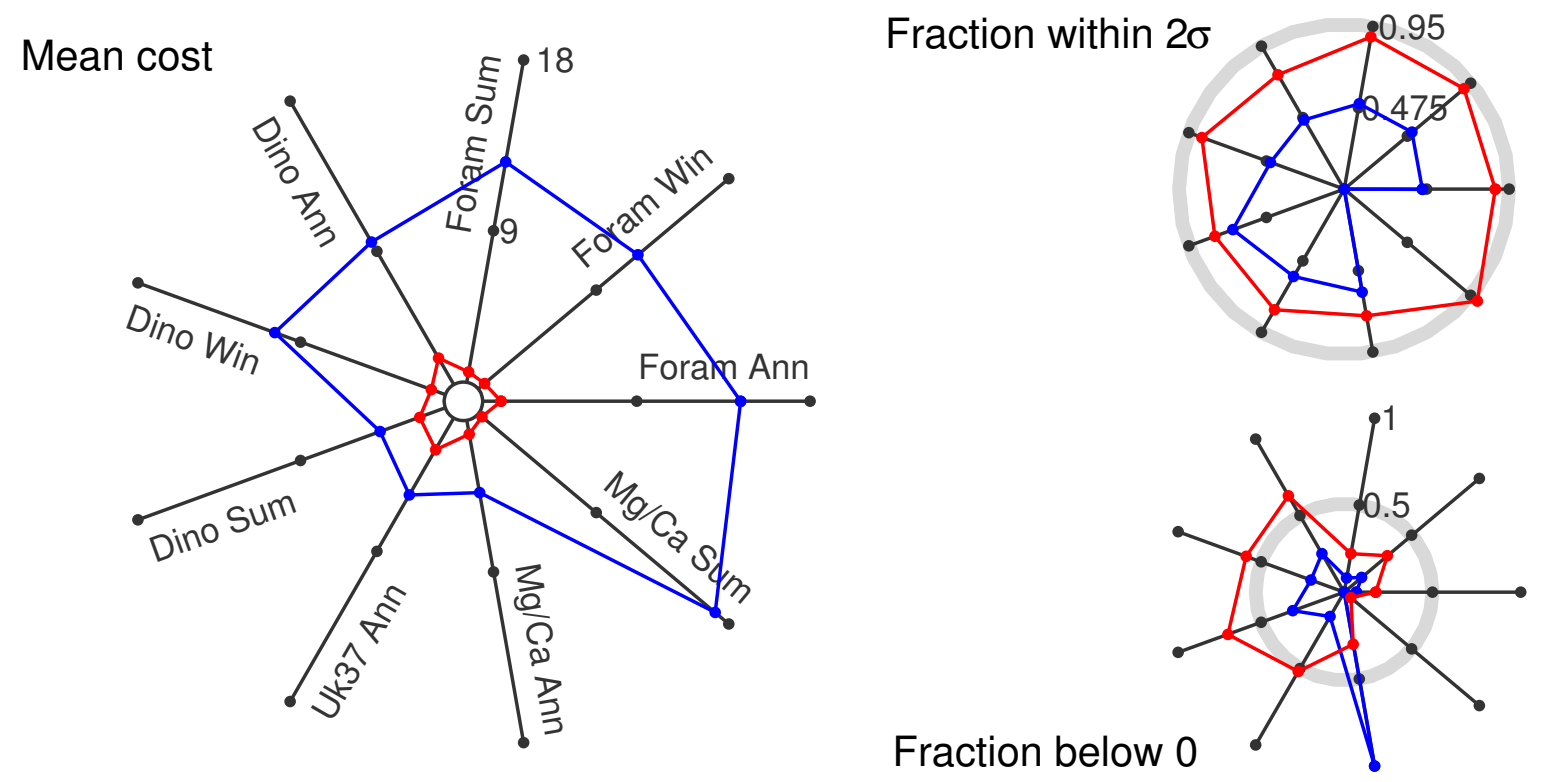

Figure 4-3: Fit of the first guess (blue) and improved LGM_AllSSTs (red) estimates against MARGO. The LGM_AllSSTs solution is improved over the first guess estimate, but is still unacceptable. The fit of the models to a given proxy and seasonality is shown on each spoke of the wheels; spokes correspond to the same data in all subplots. Solution acceptability can be evaluated as follows in this type of plot: left plot - solutions with mean cost order 1 fall near the inner circle; upper right - solutions for which $95 \%$ of the modeled values are within $2 \sigma$ of the data fall near outer circle; lower right - solutions for which half the model values are warmer and half colder fall near the ring at 0.5 (an unbiased solution). 
number of samples included, and sample reliability. Some components of the composite uncertainty may be underestimated. There are also additional uncertainties that have not been considered: whether MARGO proxies represent annual, winter, and summer conditions (as opposed to, for example, spring bloom conditions) and how well the proxies represent NSST (as opposed to, for example, the seasonal thermocline or chlorophyll maximum zone temperature). These representation errors may vary regionally, with proxy type, and with the mean climate state (i.e. glacial vs. interglacial).

2. Assumed uncertainties on initial and boundary conditions may be too small. The magnitude of control variable adjustments is directly linked to the assigned uncertainties; since the LGM initial and boundary conditions are unknown, these uncertainties are an educated guess.

3. It may be necessary to formally include model error in the computation of modeldata misfit statistics. It has been assumed that the largest model errors originate in initial and boundary conditions; these model parameters have been defined as control variables. Other model errors have been neglected under the assumption that data uncertainties are much larger than model uncertainties, an assumption that may not be valid. For example, the extent to which parameterizations of convection and eddy mixing are appropriate for the LGM Atlantic is unknown.

4. A steady state assumption may not be appropriate. The variability of the LGM Atlantic may not be well represented with a quasi-equilibrium state. Proxies could record episodic behavior, such as early spring blooms in high latitudes associated with unusually stable water column conditions [Dale, 2001]. Or it may be that interannual and longer-period variability must be incorporated to reasonably reproduce the state of the LGM Atlantic.

5. The optimization process, like all nonlinear optimizations, can become stuck in a local minimum. It is possible that an acceptable minimum exists, but that it has not 
been identified. Temporarily changing the weighting of different components in the cost function can be a useful technique for identifying alternative, and sometimes globally better, solutions. Trying alternative gradient descent algorithms is another approach to identifying alternative solutions.

That there are so many potential pitfalls and issues is an indication of the difficulties associated with paleo ocean state estimation. While it is not possible to test all of these possibilities here, some of the most interesting ones are explored in the following sections.

\subsubsection{Using subsets of data: LGM_NoForams and LGM_Forams}

The previous section indicated that the four proxy types included in LGM_AllSSTs are not consistent with each other. Two state estimates are used to test this idea: LGM_NoForams is constrained by $\mathrm{Mg} / \mathrm{Ca}$, alkenone, and dinoflagellate cyst data (foram assemblages are excluded) while LGM_Forams is constrained by foram assemblage data only. LGM_Forams is constrained by 187 independent records and LGM_NoForams is constrained by 141 independent records; note that the dinocyst, $\mathrm{Mg} / \mathrm{Ca}$, and alkenone datasets are sparse and are not evenly distributed geographically, so that state estimates constrained by any one of those datasets in isolation would be difficult to interpret.

Figure 4-4 compares the model-data fit for the LGM_NoForams estimate against the fit of LGM_AllSSTs. Excluding the foraminifera data permits the state estimate to provide a significantly improved fit to the remaining data, confirming the indication from the previous section that disagreements between the datasets contribute to the LGM_AllSSTs model-data misfits. Although LGM_NoForams fits the three included data types better than LGM_AllSSTs does, the fit to the data remains unsatisfactory: LGM_NoForams mean costs remain above one for all included datasets, less than $95 \%$ of the model values are within $2 \sigma$ of the data, and there is significant bias in the model against the data.

The LGM_Forams estimate is compared against the LGM_AllSSTs estimate in Figure 4-5. LGM_Forams shows order one mean costs, and about $95 \%$ of modeled values are within $2 \sigma$ of the foraminiferal assemblage-based data. These results are encouraging - the 
Mean Cost

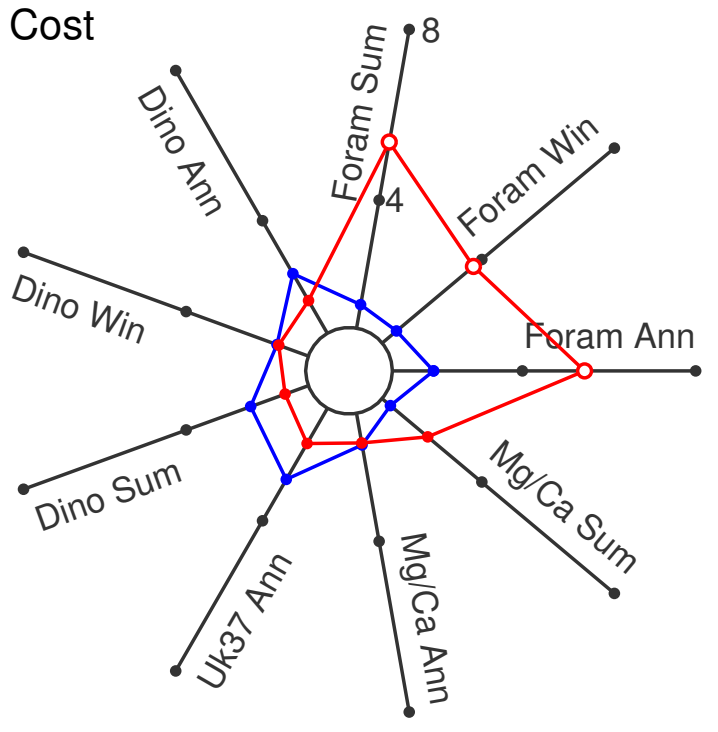

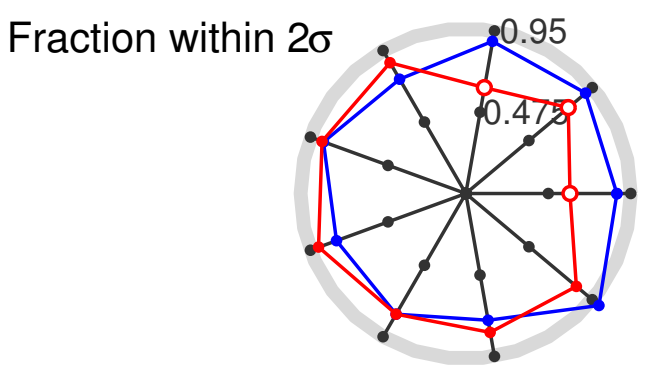

Fraction below 0

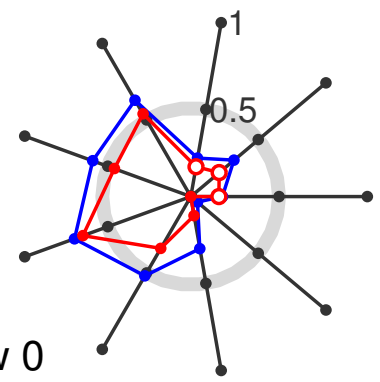

Figure 4-4: Fit of the LGM_AllSSTs (blue) and LGM_NoForams (red) estimates against MARGO. LGM_NoForams achieves an improved fit to the non-foram MARGO data on nearly all metrics (summer $\mathrm{Mg} / \mathrm{Ca}$ is an exception). The fit to excluded data (marked with open circles) is diagnostic only.

Mean Cost

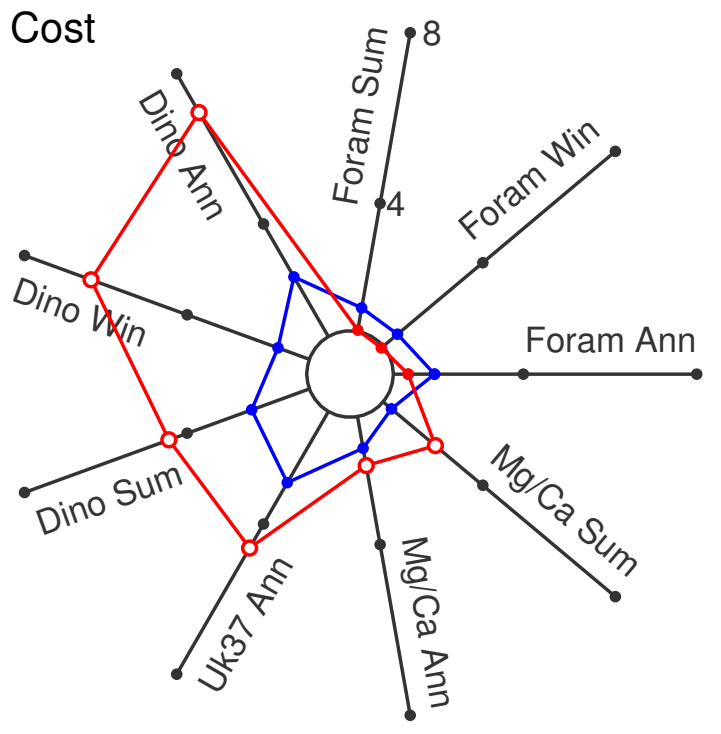

Fraction within $2 \sigma$

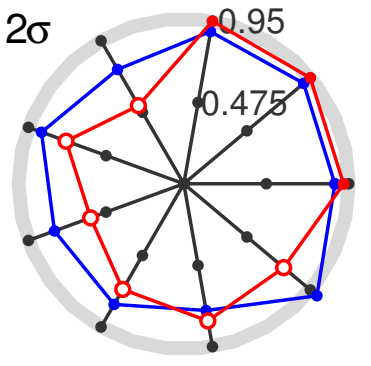

Fraction below 0

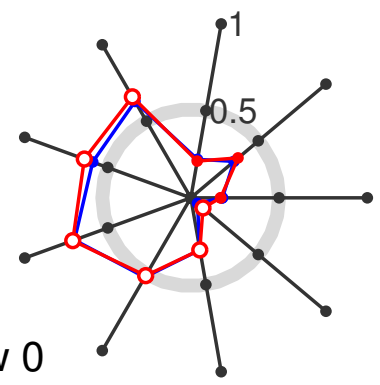

Figure 4-5: Fit of the LGM_AllSSTs (blue) and LGM_Forams (red) estimates against MARGO. Excluding non-foram data permits LGM_Forams to achieve a better fit to the foram data. The fit to excluded data (marked with open circles) is diagnostic only. 
state estimation approach is able to fit the foraminifera data within the errors assumed by the MARGO project. One aspect of the LGM_Forams solution is problematic: the model is generally too warm relative to the foram assemblage-based data, an indication that the warm bias of LGM_AllSSTs against the data was not due solely to disagreement between the datasets. This bias in model-data misfits is most likely an indication of a failure of the state estimation approach to achieve the magnitude of cooling required by the data, a possibility that is explored in the next section.

\subsubsection{Using larger atmospheric uncertainties: LGM_ForamsX2}

A potential source of the model biases observed in the estimates presented so far is that the prior uncertainties assigned to control variables are too small, leaving the state estimation approach too little latitude to make adjustments. To test this idea, a state estimate, called "LGM_ForamsX2", is developed that is constrained by foram assemblage-based data, like LGM_Forams, but for which uncertainties on atmospheric control variables are twice as large (see Table 4.1). Figure 4-6 shows that, in terms of data fit, the LGM_ForamsX2 solution is an improvement over LGM_Forams, with better foraminifera mean costs and more model values within $2 \sigma$ of the data. The fraction below 0 plot is significantly closer to 0.5 for the foraminifera data, indicating the estimate's warm bias is less pronounced. This experiment provides key evidence that the modern to LGM atmospheric changes may be larger than assumed in the LGM_AllSSTs uncertainties.

\subsection{A best estimate: LGM_Upper}

Innumerable configurations could be tested, and a variety of reasonable assumptions are likely to lead to statistically acceptable solutions. For example, using a uniform MARGO uncertainty factor of 1.5 on all data and large atmospheric uncertainties (like those in the LGM_ForamsX2 solution) may permit an acceptable solution to be found. Exploration of all possible configurations is not possible, but it is hoped that the explorations in the previ- 

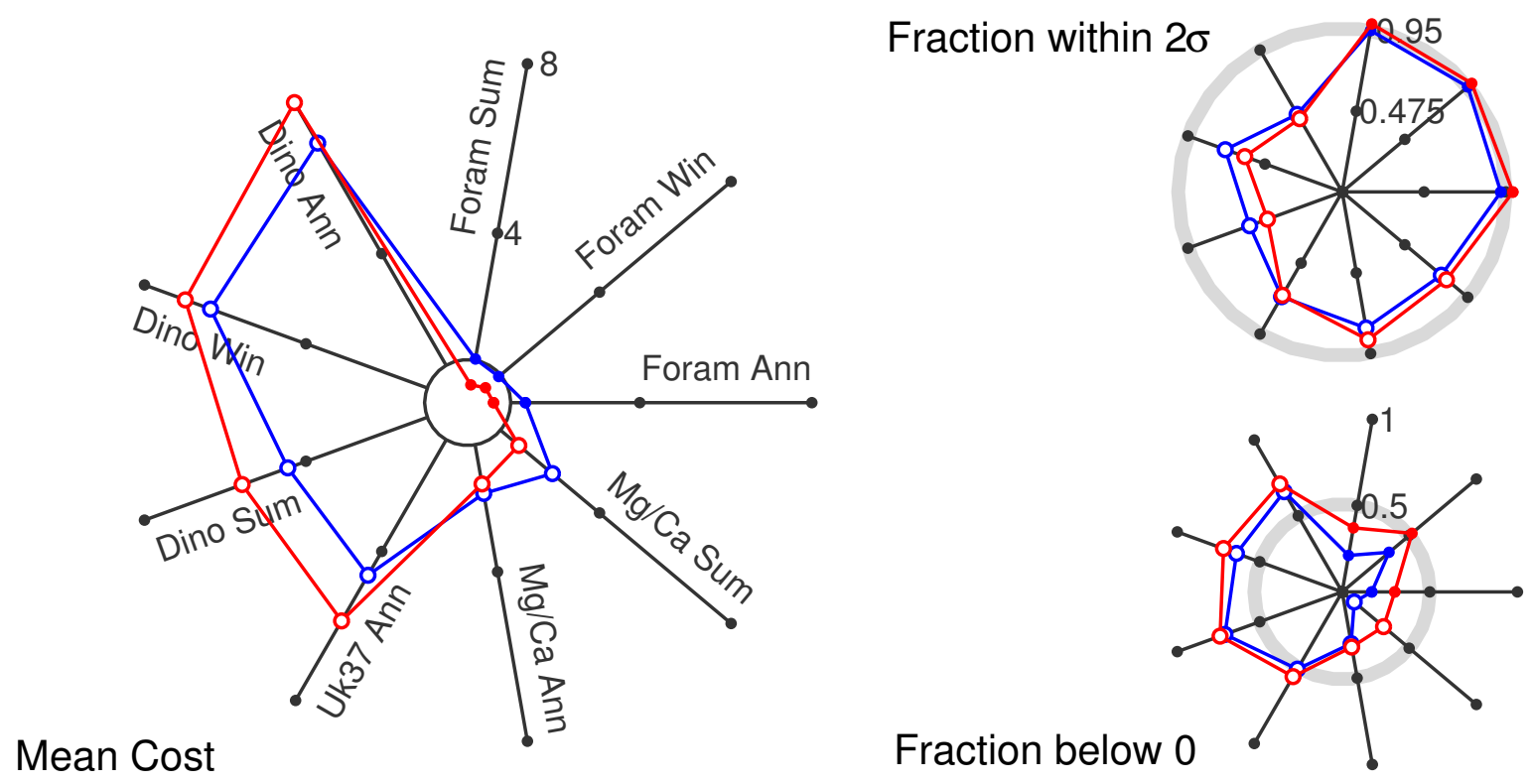

Figure 4-6: Fit of the LGM_Forams (blue) and LGM_ForamsX2 (red) estimates against MARGO. With uncertainties on atmospheric controls that are twice as large, the LGM_ForamsX2 solution achieves a better fit to the target foraminifera data. The fit to other data (marked with open circles) is diagnostic only.

ous section have given a sense of how different assumptions affect the solution. Defining what constitutes the "best" set of assumptions is as much an art as a science, and depends on the investigator's own biases. This section presents our best estimate of LGM upper ocean properties, called LGM_Upper.

\subsubsection{Assumptions}

LGM_Upper is based on the LGM_AllSSTs configuration with the following modifications; these choices are justified in the following paragraphs.

1. Uncertainty on air temperature is increased from $2^{\circ} \mathrm{C}$ to $4^{\circ} \mathrm{C}$; all other atmospheric control uncertainties are as for LGM_AllSSTs (see summary in Table 4.1).

2. Uncertainties on dinoflagellate cyst data are doubled (i.e. $f a c=2$, instead of 1 , in Equation A.1 for these data). 
3. Uncertainty on alkenone data is doubled north of $40^{\circ} \mathrm{N}$ (i.e. $f a c=2$ ).

These assumptions are taken for the following reasons. The LGM_ForamsX2 estimate showed that permitting the system to make large atmospheric adjustments helped reduce the warm model bias. Are such large atmospheric adjustments truly required by the data? That is, does an acceptable solution exist with smaller atmospheric adjustments? For air temperature, it seems probable that the data do indeed require these larger uncertainties: this is the most direct way to address a warm model bias and, in hindsight, the LGM_AllSSTs uncertainty of $2^{\circ} \mathrm{C}$ seems overly restrictive as compared to the often $>2^{\circ} \mathrm{C}$ NSST changes reconstructed by the proxies. The LGM_Upper configuration is based on the hypothesis that the smaller uncertainties of LGM_AllSSTs are sufficient in general, but that the air temperature uncertainty must take the larger value used in LGM_ForamsX2.

Proxy uncertainties are modified to address the large disagreement between the proxy types north of $45^{\circ} \mathrm{N}$. In this region, either uncertainties are too small, or one or more of the proxy types actually records conditions other than winter/summer/annual mean NSST. Rather than excluding some data altogether, the uncertainties are increased on the alkenone and dinocyst data north of $40^{\circ} \mathrm{N}$. This modification, which is equivalent in practice to downweighting these data, is done for the following reasons. The alkenone data are downweighted because (1) alkenones are thought to record warm season temperatures in colder regions [Schneider et al., 2010], as opposed to the annual mean, as reported in MARGO; and (2) the sensitivity of the calibration is low at cold temperatures [Conte et al., 2006].

Dale [2001] note a variety of serious concerns regarding reconstructions of past ocean conditions with dinocysts: that in cold regions, dinoflagellates survive the winter in a dormant cyst phase, and thus are unlikely to record characteristics of winter-time conditions; that dinocysts are small and easily transported over long distances, so that many of the cysts in sediment samples may have originated in distant ocean regions; and that many of the species used for reconstruction have broad tolerances for temperature, salinity, and sea ice, limiting their utility in reconstructing these conditions. Based on some of these same concerns, Telford [2006] re-analyzed the same modern training set and LGM dinoflag- 
ellate cyst assemblage data as used in the MARGO analysis of De Vernal et al. [2005], and found significant issues affecting interpretation of these data. Of particular note here, Telford [2006] find that MARGO uncertainty estimates on dinocyst LGM NSST estimates are probably much too low, and that there is little evidence that winter-time NSSTs can be independently estimated from these data.

\subsubsection{Consistency of the state estimate}

This section focuses primarily on the extent to which the LGM_Upper estimate is consistent (or not) with the MARGO NSST data, given prior uncertainty assumptions. At the end of the section, the consistency of the control variable adjustments with the prior uncertainty assumptions is also evaluated. This section does not present analyses such as maps of LGM_Upper properties or maps of control variable adjustments; this type of model state analysis will be presented in Section 4.4.3.

Fit to MARGO NSSTs data. As shown in Figure 4-7, the fit of LGM_Upper to the MARGO NSST data is acceptable: for all datasets, mean costs are order one and about $95 \%$ of model values are within $2 \sigma$ of the data. The state estimate is able to more closely match the foram assemblage, $\mathrm{Mg} / \mathrm{Ca}$, and low-latitude alkenone data by relaxing the fit to the high-latitude alkenone and dinocyst data. The fraction below 0 subplot in Figure 4-7 shows that LGM_Upper is generally too warm relative to the foram assemblage and $\mathrm{Mg} / \mathrm{Ca}$ data, and too cold relative to the dinocyst data.

Figure 4-8 provides a latitudinal analysis of the misfit of the LGM_Upper estimate against MARGO as compared to the WOA'09 misfits against MARGO. The WOA'09 misfits show strong latitude-dependence. For the foraminiferal assemblage data and, to some extent, for the $\mathrm{Mg} / \mathrm{Ca}$ data, the state estimate has successfully reproduced this latitudinal dependence so that the LGM_Upper misfits for these two datasets are not strongly latitudedependent. Figure 4-8 makes clear that the remaining biases between datasets north of $45^{\circ} \mathrm{N}$ cannot be simply corrected by adding an offset to one or more datasets. Particularly in the high-latitudes, the proxies disagree not only on the mean difference in temperature 

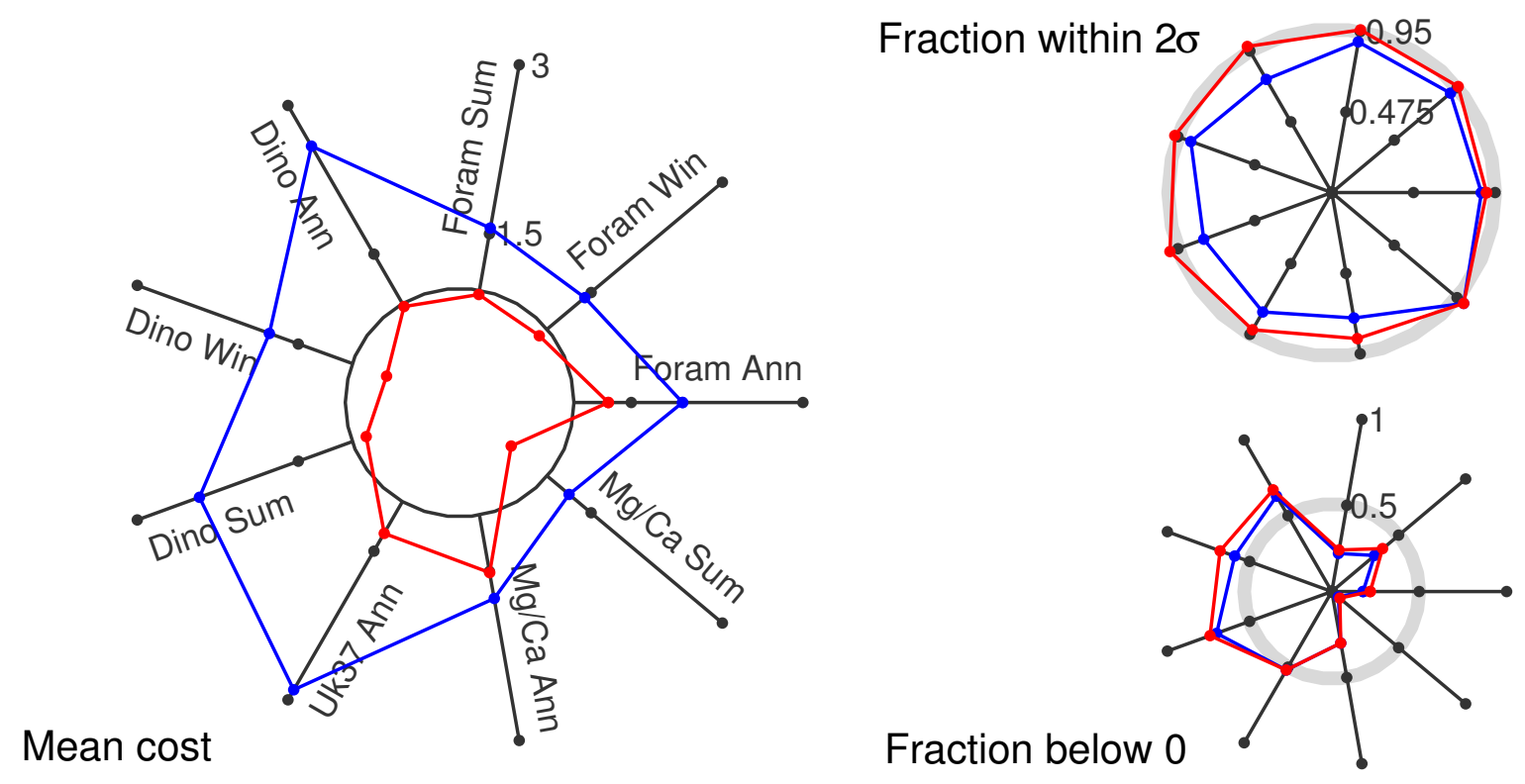

Figure 4-7: Fit of the LGM_AllSSTs (blue) and LGM_Upper (red) estimates against MARGO. The LGM_Upper estimate is statistically acceptable. Much of the remaining bias in the model against the datasets is due to the model compromising between datasets that disagree.

between today and the LGM, but also on the latitudinal gradient of change between the modern and LGM NSSTs.

Maps of normalized misfits for LGM_Upper are shown in Figure 4-9 and are herein discussed, starting from the north. North of about $60^{\circ} \mathrm{N}$ in the GIN Seas, normalized misfits are order one for the two datasets with coverage in this region: foraminifera and dinocysts. In the subpolar gyre $\left(45-60^{\circ} \mathrm{N}\right)$, all four datasets are represented. Normalized misfits remain unacceptable in the core of this region southwest of Iceland - a two-fold increase in alkenone and dinocyst uncertainties in this region is not sufficient to permit an acceptable fit to the data. Misattribution of one or more proxies to seasonal NSSTs and/or other unaccounted for errors appear to be especially severe in this region.

South of $45^{\circ} \mathrm{N}$, normalized misfits are dominated by east-west patterns (Figure 4-9). In the western half of the basin, there is unfortunately very little non-foraminifera data, and in a large region off the east coast of N. America, there is little data of any type. Misfits to the 

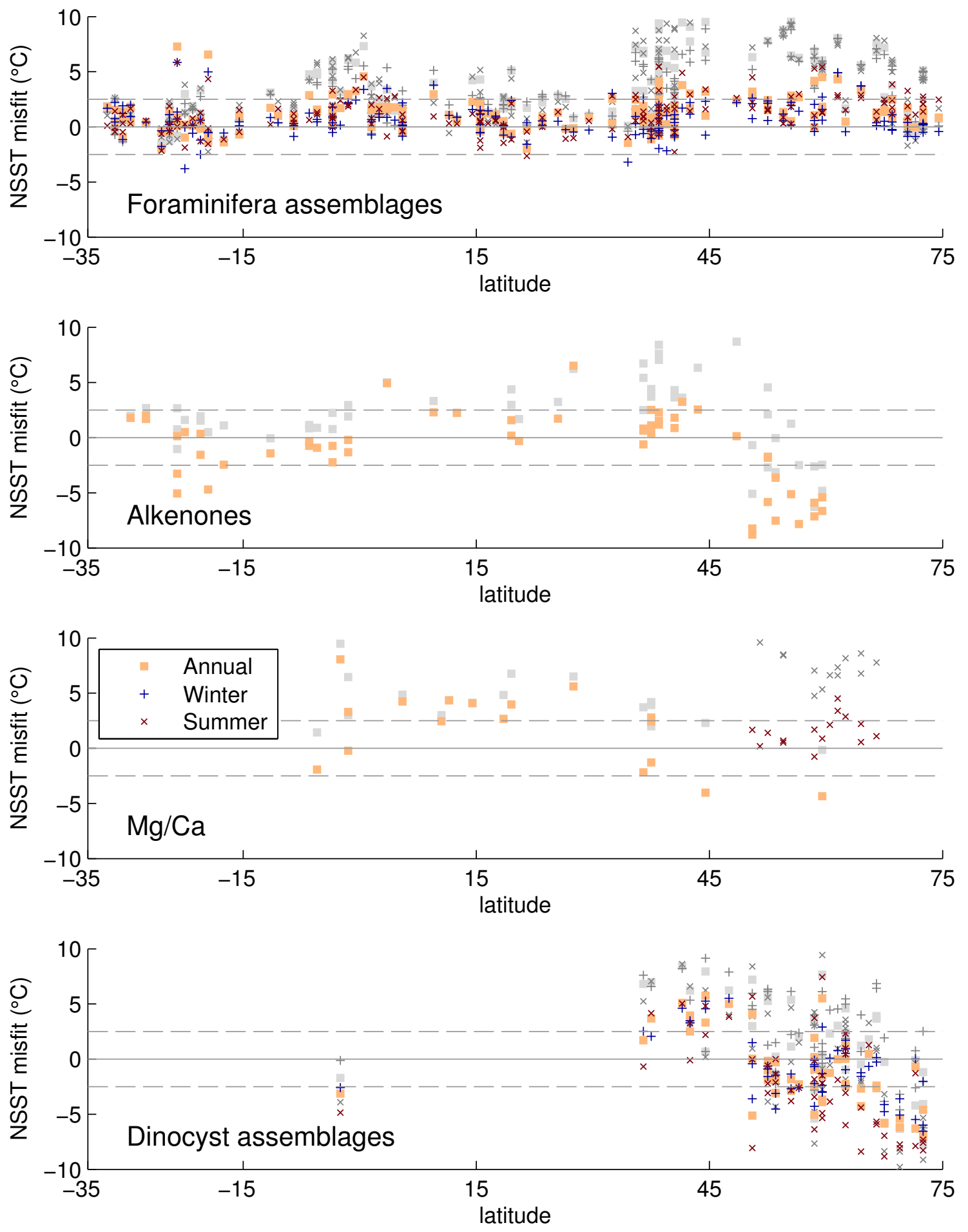

Figure 4-8: LGM_Upper - MARGO NSST misfit in ${ }^{\circ} \mathrm{C}$ (colored markers) as compared to WOA'09 - MARGO NSST misfit (gray markers) as a function of latitude. Dashed lines at $\pm 2.5^{\circ} \mathrm{C}$ are included as a visual reference (see Figure A-1 for distribution of actual MARGO uncertainties). 
Annual mean
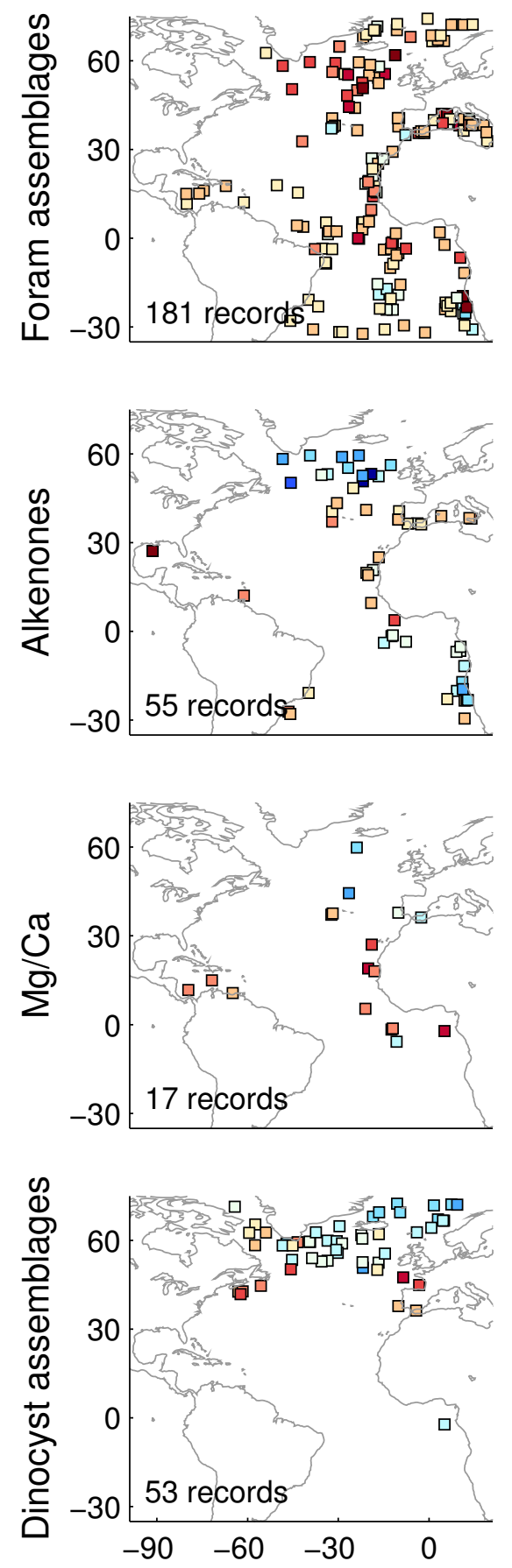

Winter mean
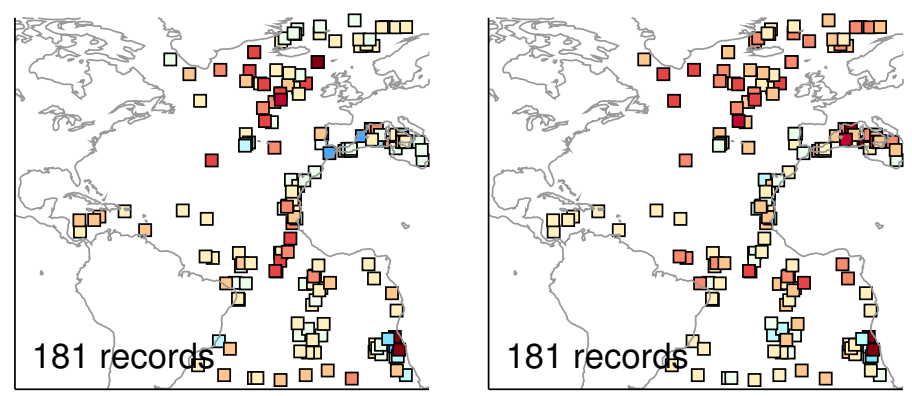
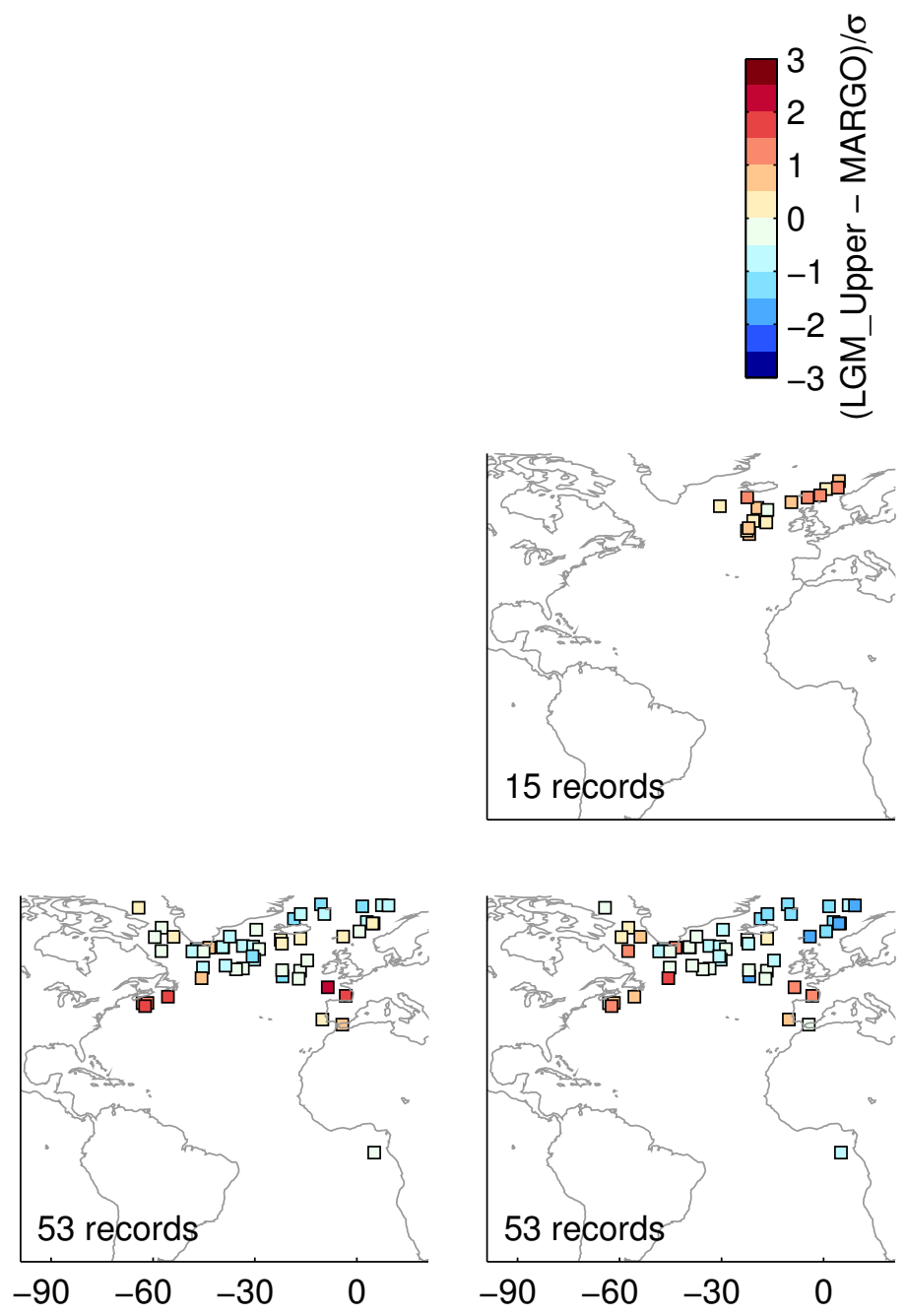

Figure 4-9: Normalized misfits of the LGM_Upper solution to the MARGO NSST proxies. Values near the range of -1 to +1 indicate LGM_Upper is within assumed uncertainties of the MARGO data (the desired result). 
few available data are generally acceptable in these western regions, with a few exceptions: the model is too warm relative to a lone alkenone point in the Gulf of Mexico and relative to dinoflagellate cyst data in the Gulf Stream separation region. The Mediterranean Sea is dominated by foram assemblage records; although the fit is statistically acceptable, it is worth noting that the model fits the winter foraminifera temperatures especially well while the model remains too warm in the annual mean and summer mean comparisons.

In the rest of the eastern half of the basin a better distribution of proxy types is available and there are no similarly large gaps in coverage. The model is generally able to reproduce increased LGM cooling in the eastern basin, as observed in the data. Normalized misfits are acceptable, with the following exceptions. As shown in Figure 2-3, the MARGO data are consistent with strong LGM cooling in the eastern equatorial region $\left(8^{\circ} \mathrm{S}\right.$ to $\left.8^{\circ} \mathrm{N}\right)$; Figure 49 shows that LGM_Upper is not able to reproduce this change. There are likely one or more model deficiencies in this region that makes such a shift infeasible in the model; equatorial dynamics are challenging, and particularly so for low resolution models (see discussion of PMIP2 challenges in this region in Section 2.3.1).

The upwelling regions off the coast of Africa around $10-30^{\circ} \mathrm{S}$ and $10-30^{\circ} \mathrm{N}$ also show particularly large LGM changes in the data (see Figure 2-3). As shown in Figure 4-9, the northern upwelling region is too warm in LGM_Upper relative to the annual and winter mean foraminifera data and relative to the $\mathrm{Mg} / \mathrm{Ca}$ annual mean data, but is acceptable relative to the summer mean foraminifera data. In the southern upwelling region, the MARGO foraminifera data show an abrupt transition from small reconstructed LGM shifts around $30^{\circ} \mathrm{S}$ to order $10^{\circ} \mathrm{C}$ shifts around $20^{\circ} \mathrm{S}$ (see Figure 2-3). The alkenone data show small LGM NSST changes throughout this region. Given these disagreements between the proxy types, large normalized misfits are to be expected in this $10^{\circ} \mathrm{S}-30^{\circ} \mathrm{S}$ upwelling zone.

Consistency of control variable adjustments. Figure 4-10 shows cumulative distribution functions for normalized adjustments to atmospheric control variables for LGM_Upper, where normalized adjustments are given by (adjusted control - prior control)/ $\sigma$. Figure 411 shows the same for initial and southern boundary condition control variables. Prior 

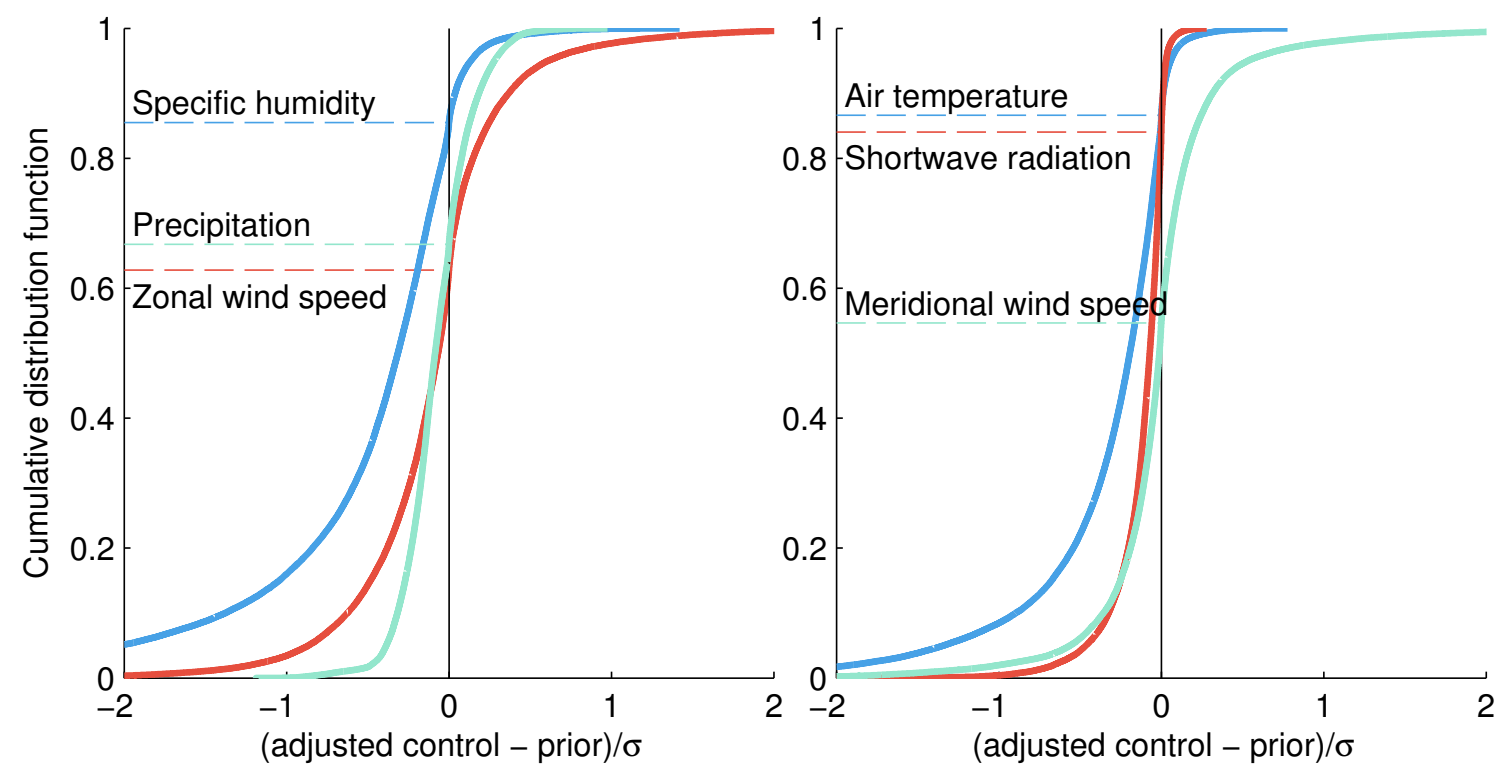

Figure 4-10: Cumulative distribution functions for adjustments to LGM_Upper atmospheric control variables, normalized by prior uncertainty assumptions (final control - prior control) $/ \sigma$. Horizontal lines indicate what fraction of values fall below zero. For most variables, nearly $100 \%$ of the adjusted control variables fall within $\pm 2 \sigma$ of prior estimates; for specific humidity the value is $95 \%$.

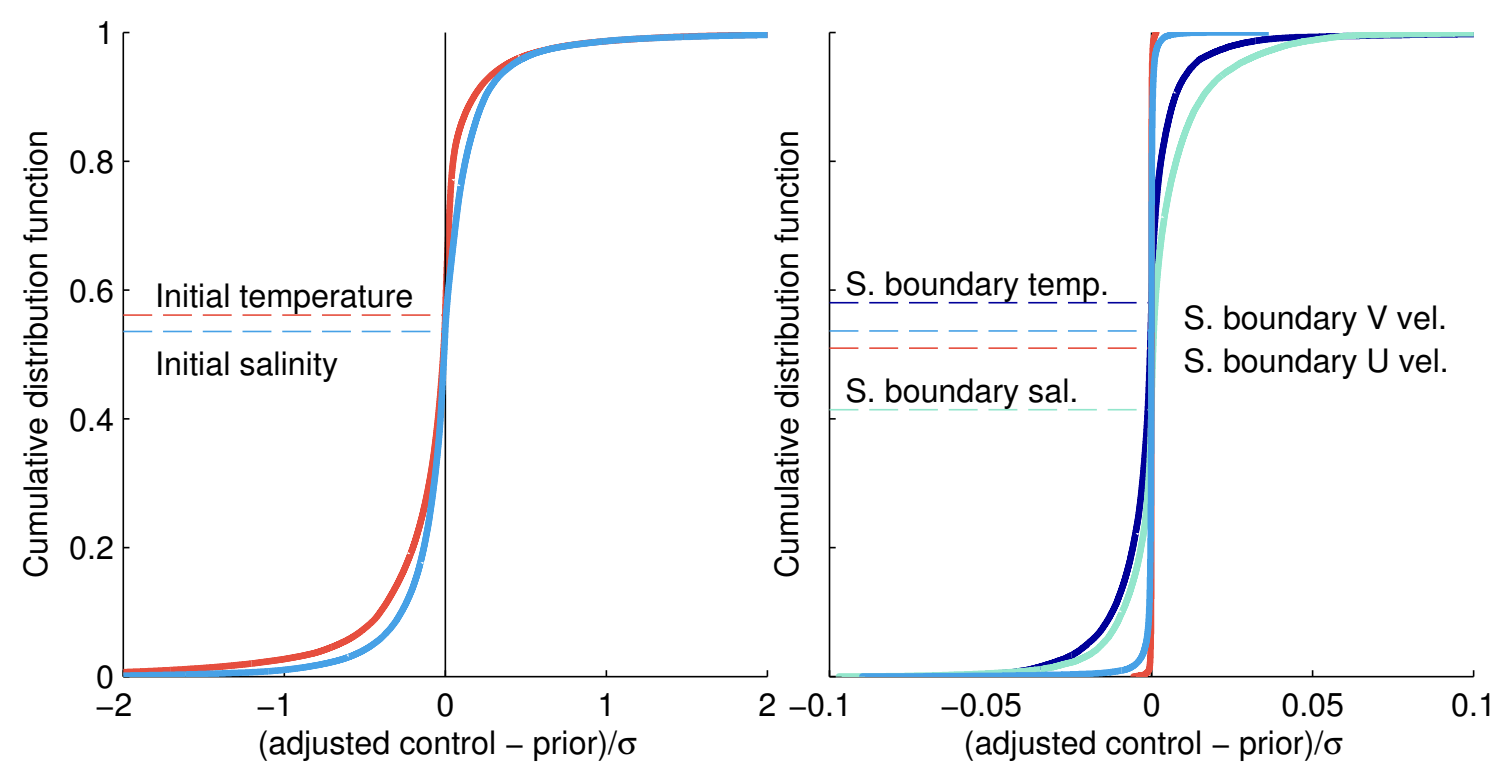

Figure 4-11: Cumulative distribution functions for adjustments to LGM_Upper initial conditions (left) and to conditions at the southern boundary of the model (right); all are normalized by prior uncertainty assumptions. Note the expanded axis on the right. 
uncertainty estimates were described in Section 4.2. For every control variable shown in these two figures, $95 \%$ or more of the adjusted controls are within $\pm 2 \sigma$ of the prior control variables. Significant bias is seen in the atmospheric control variables, a property that is to be expected here - Atlantic Ocean scale changes are to be expected in moving from a modern climate (the prior atmospheric forcing) to an LGM-like climate. Most air temperature and shortwave radiation adjustments are negative, indicating large-scale cooling was required to reach a more LGM-like climate. Precipitation and specific humidity changes are also largely negative: large-scale drying occurred in the model in moving from a modern state to the LGM_Upper state. That adjustments to the initial temperature distribution do not show a strong bias is interesting: perhaps the $3^{\circ} \mathrm{C}$ shift from modern conditions used to generate first guess initial conditions was appropriate. As was the case for the Modern_TS solution (see Figure 3-3), control adjustments for southern boundary conditions are very small relative to prior uncertainty assumptions. The same argument can be made here as was made there: most of the model properties are not affected by the southern boundary conditions in the course of a short simulation such as the 10 years used here.

\subsubsection{Analysis of solution}

In the previous section, we showed that the LGM_Upper estimate achieves an acceptable fit to the MARGO data in most regions, and that the control variable adjustments are consistent with prior uncertainty assumptions. This section focuses on analysis of model properties such as near sea surface temperature, sea ice extent, atmospheric forcing, and volume transports. Near sea surface temperature can be thought of as a mapped or interpolated field, while other analyzed properties are inferences based on model dynamics and the incorporated data records. This analysis can be compared to the following figures of similar properties for the Modern_TS solution: Figures 3-5 (annual mean NSST), 3-10 (MOC and transports at $29^{\circ} \mathrm{N}$ ), and 3-12 (sea ice distribution). Note also that additional analyses are describe in Section 4.5, where the LGM_Upper estimate will be compared to PMIP2 model results. 

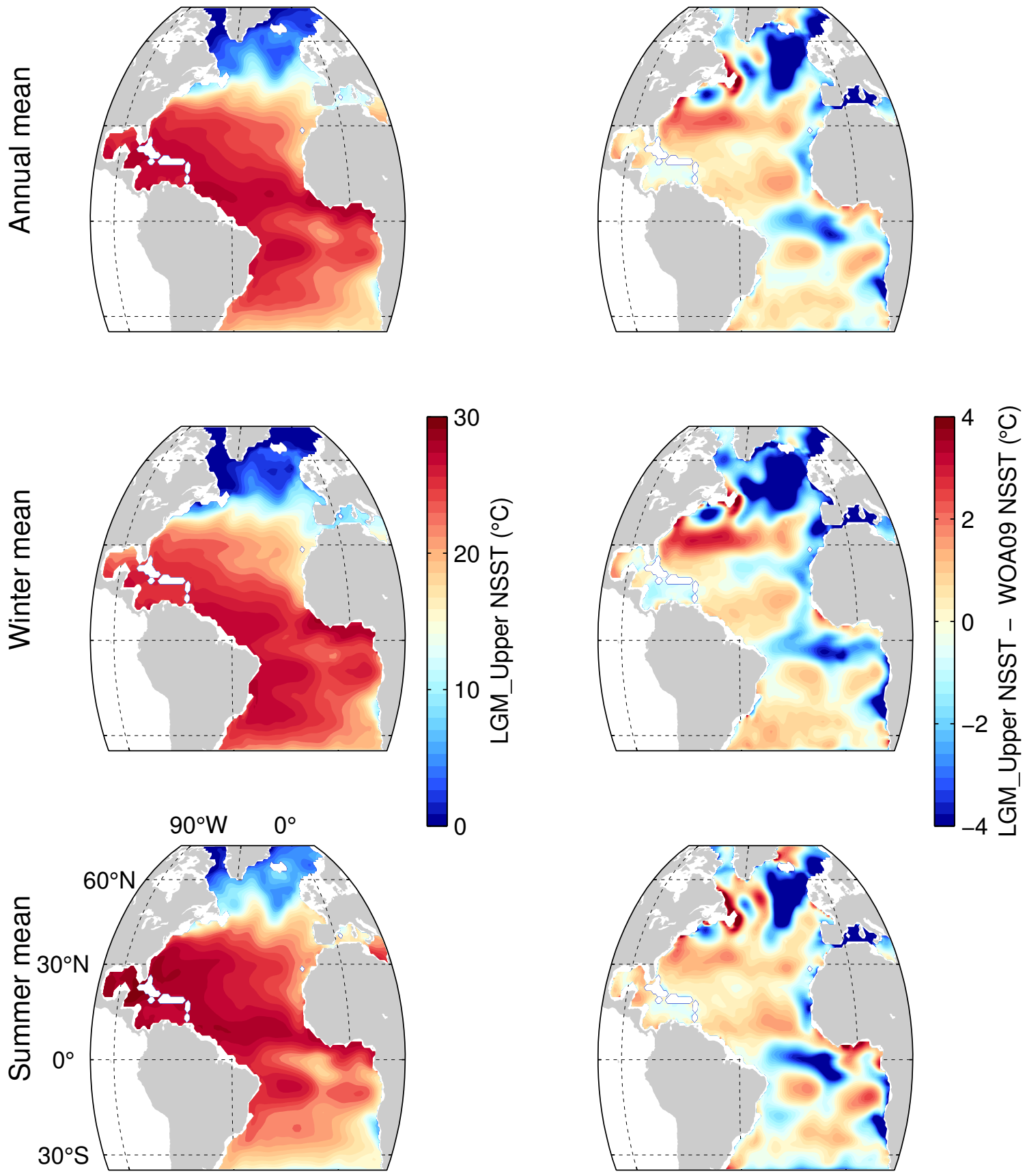

Figure 4-12: Mean near sea surface temperature (NSST, mean over the top 30 meters) for LGM_Upper (left) and difference with WOA'09 (right). Rows show annual mean (top), winter mean (middle), and summer mean (bottom). 
Near sea surface temperatures. Figure 4-12 shows LGM_Upper annual, winter and summer mean NSSTs and the difference between LGM_Upper and WOA'09. All three anomaly plots show increased zonal NSST gradients in the LGM solution relative to the modern; these changes are associated with relatively strong cooling along the eastern boundary. There is a consistent pattern of LGM warming around $30^{\circ} \mathrm{N}$ and relatively intense cooling in the subpolar gyre, leading to a strong increase in the meridional NSST gradient in the mid-latitudes. The increased zonal and meridional NSST gradients in LGM_Upper are strongest in the winter mean fields.

Sea ice fraction. The LGM_Upper sea ice fraction and the difference from Modern_TS are shown in Figure 4-13. The August sea ice extent is minimal in both LGM_Upper and Modern_TS, though there is slightly more sea ice in LGM_Upper. In February, LGM_Upper has significantly more sea ice than Modern_TS. This increase is largely consistent with a southward shift of the sea ice edge without a major re-organization of the sea ice distribution.

As discussed in Chapter 2, sea ice proxies are not as well-tested as NSST proxies and many open questions remain. Nonetheless, a qualitative comparison is worthwhile. Figure 4-14 compares the sea ice extent in LGM_Upper against MARGO sea ice reconstructions based on foraminifera and dinocysts (see also Figure 2-5 for a summary picture of these sea ice reconstructions). The model field is the sea ice fraction in February and August, as in Figure 4-13. The foram-based sea ice estimate is reported in MARGO as "presence" or "no presence" for JFM and JAS; these fields are mapped directly in Figure 414. The MARGO dinocyst sea ice estimates are reported in months per year of sea ice coverage of at least 50\%. For Figure 4-14, winter (summer) sea ice is defined to be coverage greater than 2 (10) months; note that the resulting maps are sensitive to the cutoffs used for winter/summer. LGM_Upper and the proxies show limited summer sea ice, indicating relatively mild summer conditions in the high-latitude N. Atlantic at the LGM. In winter, the data are highly variable with proxies consistent with either sea ice presence or absence sprinkled through the high-latitude N. Atlantic. 


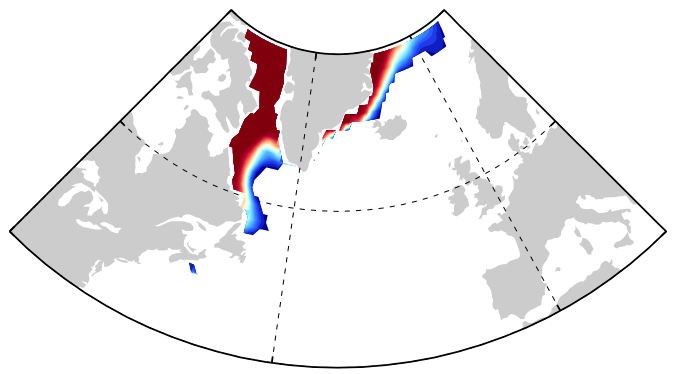

LGM_Upper, February

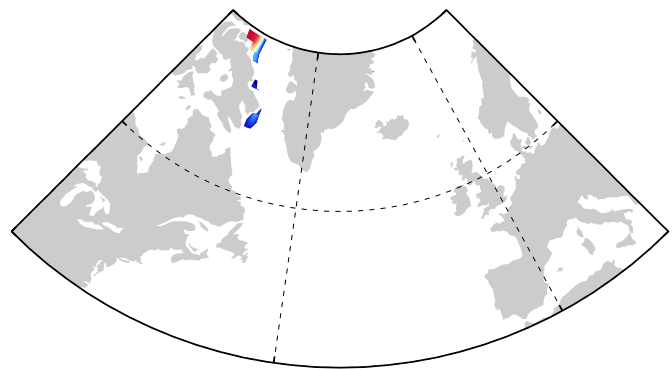

LGM_Upper, August
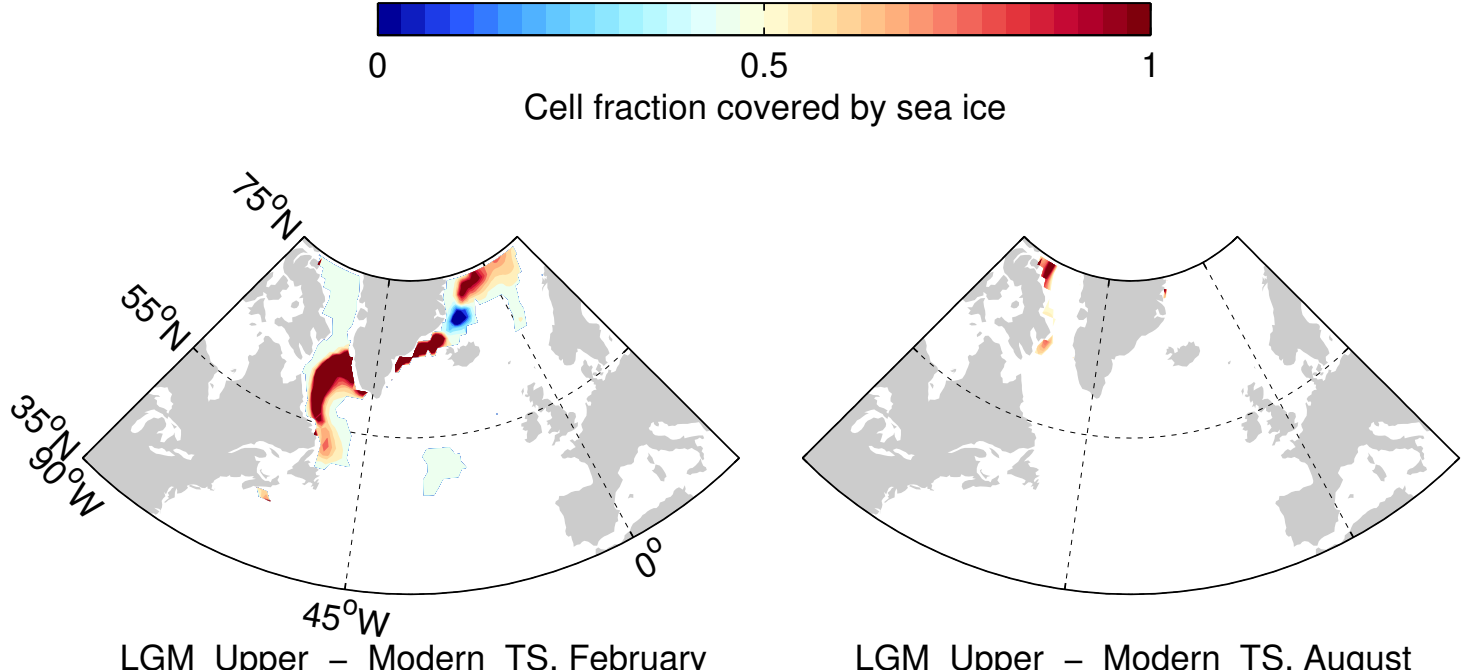

LGM_Upper - Modern_TS, February

LGM_Upper - Modern_TS, August

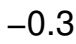

0

0.3

Change in sea ice fraction

Figure 4-13: LGM_Upper cell fraction covered by sea ice in February and August (top row) and difference from Modern_TS (bottom row). Sea ice fractions below 0.02 are not plotted to avoid the implication of substantial sea ice where there is not. The LGM_Upper sea ice extent is somewhat more extensive than modern, especially in winter.

Taken as a whole however, LGM_Upper and the data agree on winter time sea ice cover in the Labrador Sea, the model agrees with the foraminifera reconstruction of sea ice presence off the northeast coast of Greenland (though the dinocyst reconstruction indicates an absence of sea ice), and in the central subpolar gyre both data types indicate a greater sea ice extent than does LGM_Upper. The uncertainties on atmospheric adjustments used to 

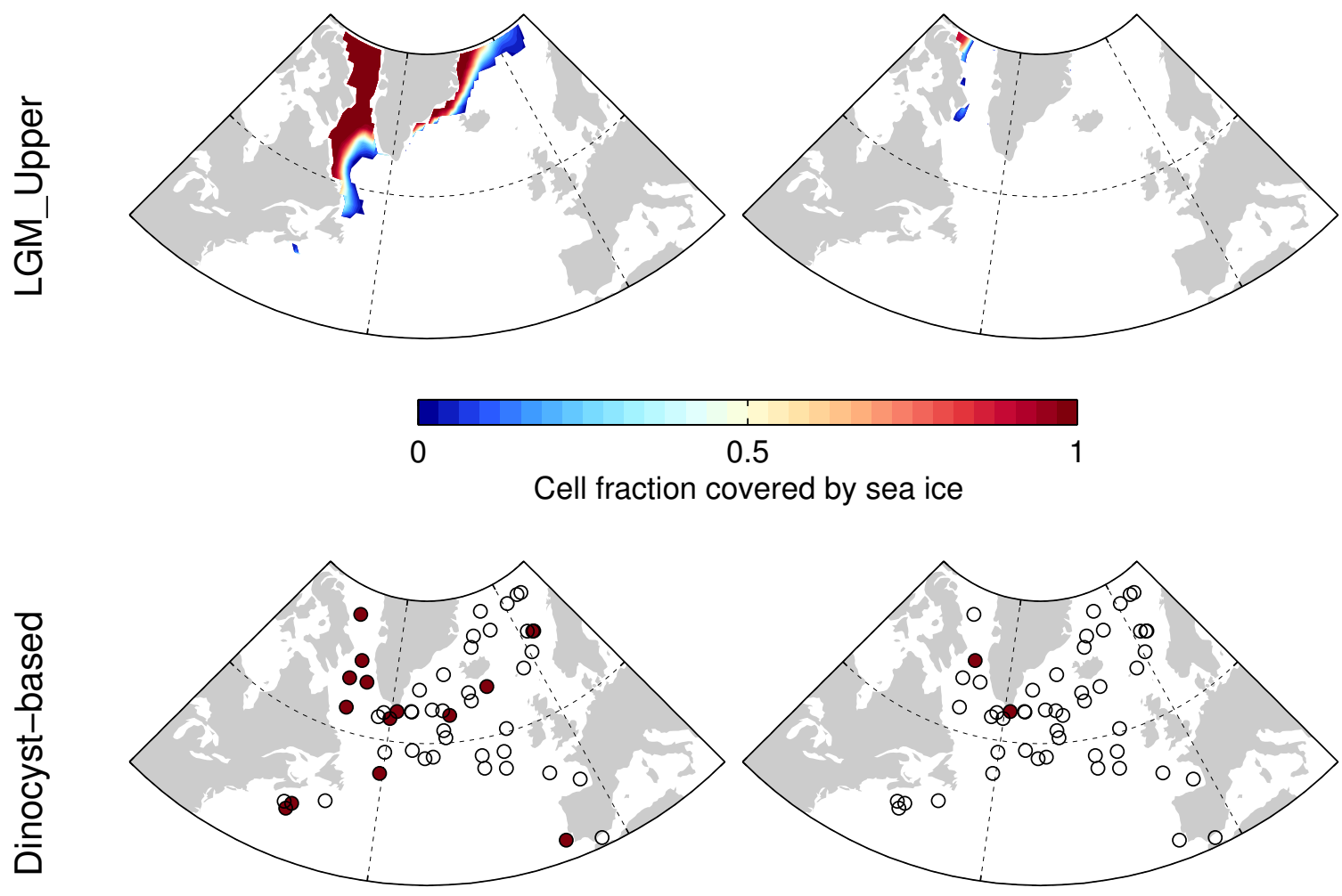

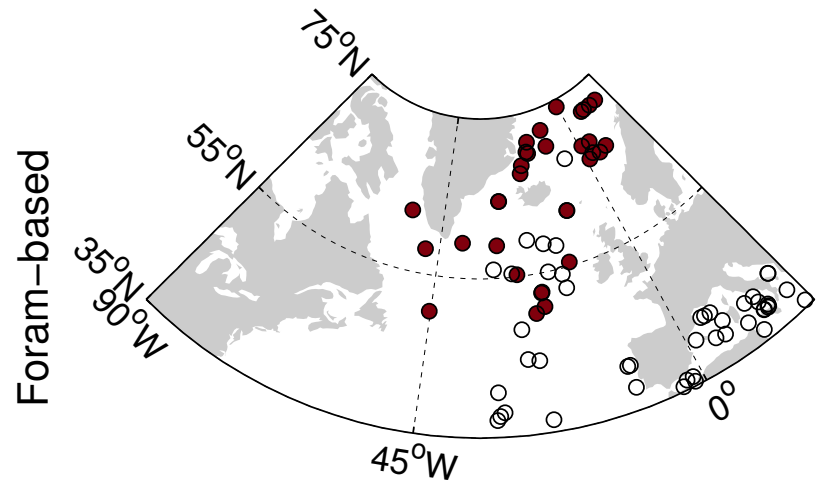

Winter

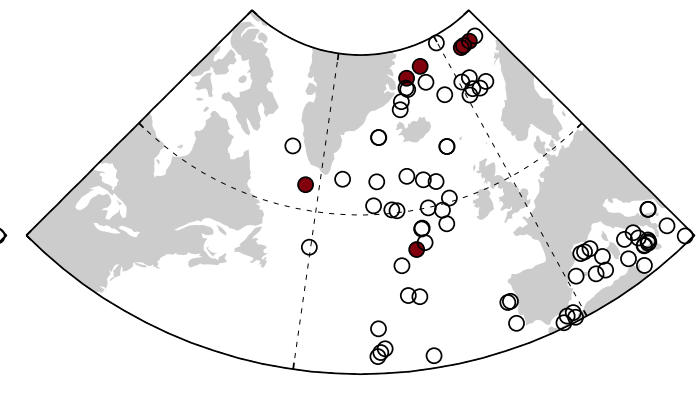

Summer

Figure 4-14: Comparison of sea ice fraction in LGM_Upper (top) and estimates of sea ice presence based on dinocysts (middle) and foraminifera (bottom) for winter (left) and summer (right). In the top plot, sea ice fractions below 0.02 are not plotted to avoid the implication of substantial sea ice where there is not. In proxy plots, red indicates sea ice presence, empty circles indicate sea ice absence; see text for definitions of sea ice presence for the proxies. 

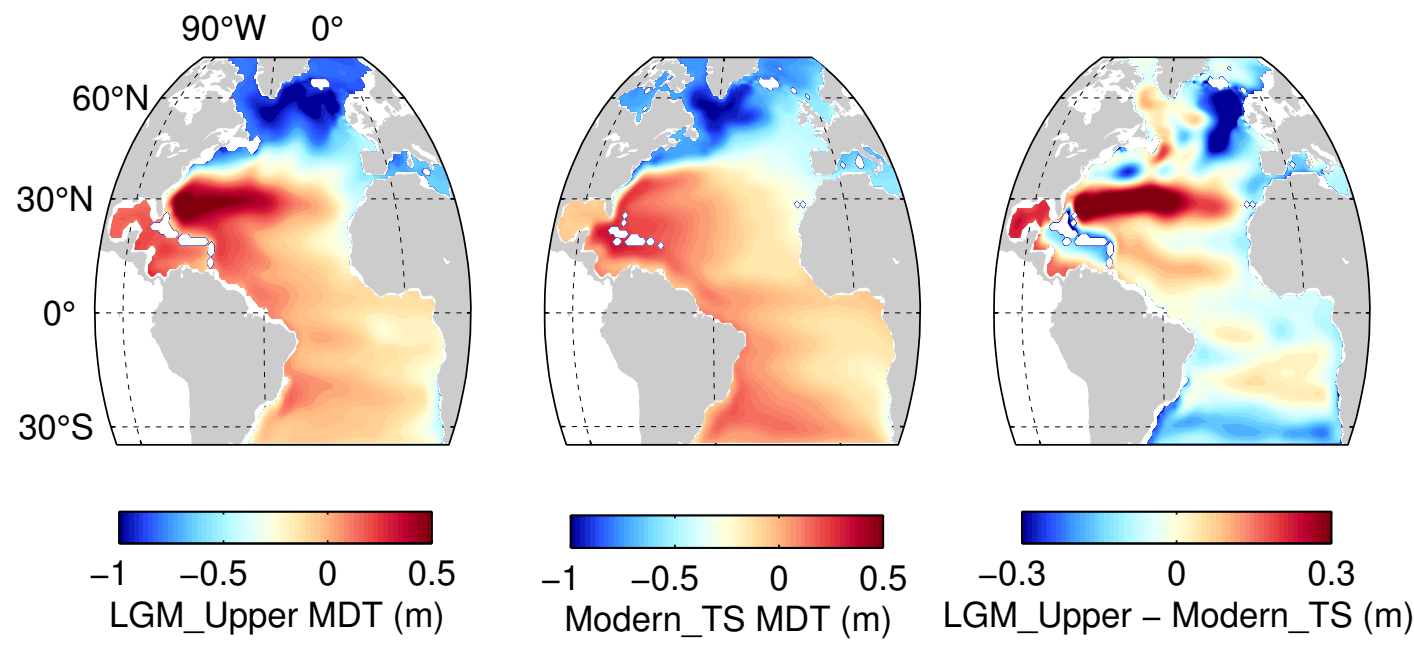

Figure 4-15: Mean dyamic topography (MDT) in meters for LGM_Upper (left) and for the Modern_TS estimate (center). Differences are shown on the right.

develop the LGM_Upper estimate are conservative; that the sea ice extent in LGM_Upper may be too limited in the subpolar gyre is an indication that these uncertainties may have been too restricted in this region. Indeed, moving from a scenario without sea ice to one with sea ice can require enormous shifts in air temperature and heat fluxes; improving the choice of atmospheric control uncertainties (and understanding the sensitivity to those choices) is an interesting avenue for future investigation.

Mean dynamic topography, transports, and the AMOC. Mean dynamic topography (MDT) was discussed in Chapter 3 as a useful representation of the long term mean circulation (see Figure 3-9 and surrounding discussion). Figure 4-15 compares MDT in LGM_Upper against that from Modern_TS. Relative to the Modern_TS estimate, the LGM_Upper MDT is more strongly elevated in the subtropical gyre and is more strongly depressed in the subpolar gyre; these changes indicate an intensified gyre circulation. The subpolar gyre is also expanded eastward in LGM_Upper relative to Modern_TS; this is the dynamical shift employed by the state estimate to provide an improved fit to the very cold MARGO NSST temperatures in this region (see Figure 2-3).

Since the deep ocean is not equilibrated in the 10-year LGM_Upper estimate, the AMOC 
and deep ocean transports are somewhat dependent on initial conditions (though these transports do satisfy geostrophy and the other dynamical equations of the model). With these limitations in mind, meridional transports in LGM_Upper and OCCA can be compared (Figure 4-16). The LGM_Upper AMOC is shallow (negligible net flow below 2500 $\mathrm{m}$ ) and weak (maximum overturning of about $8 \mathrm{~Sv}$ ). Weak DWBC transport in LGM_Upper is in line with the reduced AMOC strength. The surface wind-driven circulation is substantially stronger in LGM_Upper than in the modern circulation, with most of the Gulf Stream transport returned at the surface, rather than at depth. This arrangement is in contrast to an assumption sometimes seen that the Gulf Stream or Florida Straits transport is related in a direct way to AMOC strength (e.g. Lynch-Stieglitz et al. [1999b]). Further discussion of the deep circulation is postponed to Chapter 5 .

Atmospheric control variable adjustments. The modern to LGM ocean property changes described in the preceding paragraphs are produced by control adjustments in the model's initial conditions, southern boundary conditions, and atmospheric forcing. Since plotting all of the control variables is not practical, we focus on atmospheric adjustments; these adjustments dominate the surface ocean circulation so are most relevant to the focus of this chapter. As shown in the top row of Figure 4-17, LGM_Upper shows large regions of enhanced LGM wind speed with minimal regions of decreased wind speed. Upwellingfavorable winds are increased in the eastern equatorial zone and in upwelling zones off the coast of Africa. An increase in strength of the Northern Hemisphere westerly and easterly winds implies an increased wind-driven circulation, consistent with the transports reported in Figure 4-16. LGM_Upper air temperatures (Figure 4-17, middle row) show intense cooling of $3-5^{\circ} \mathrm{C}$ in the eastern low-latitudes and in the subpolar gyre; elsewhere, differences between modern and inferred LGM air temperatures are smaller than $1-2^{\circ} \mathrm{C}$.

Given the lack of reliable LGM salinity data, evaporation and precipitation are poorly constrained. Nonetheless, the rates of evaporation and precipitation are affected by the control adjustments, and should be understood as they impact ocean circulation through salinity. Figure 4-17 (bottom left) shows that LGM_Upper has a conventional pattern of 
OCCA MOC and transports at $29^{\circ} \mathrm{N}$
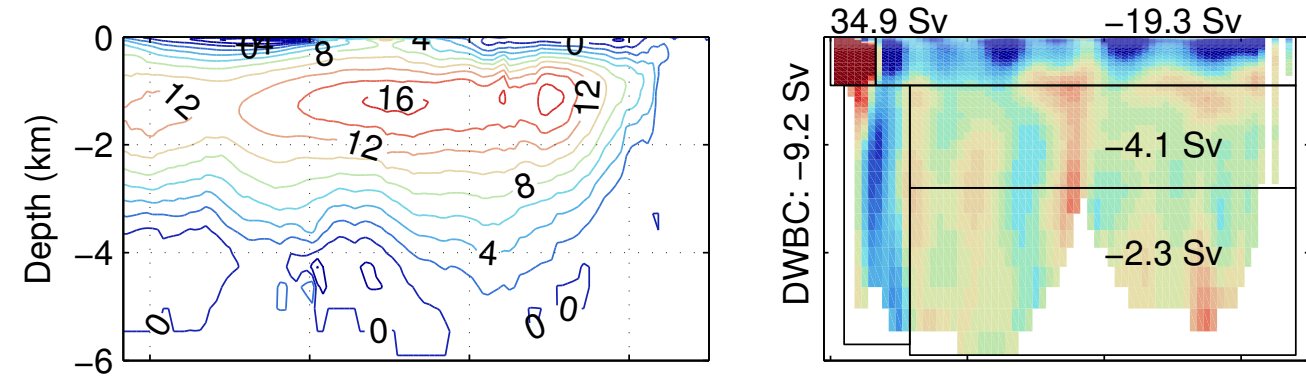

LGM_Upper MOC and transports at $29^{\circ} \mathrm{N}$
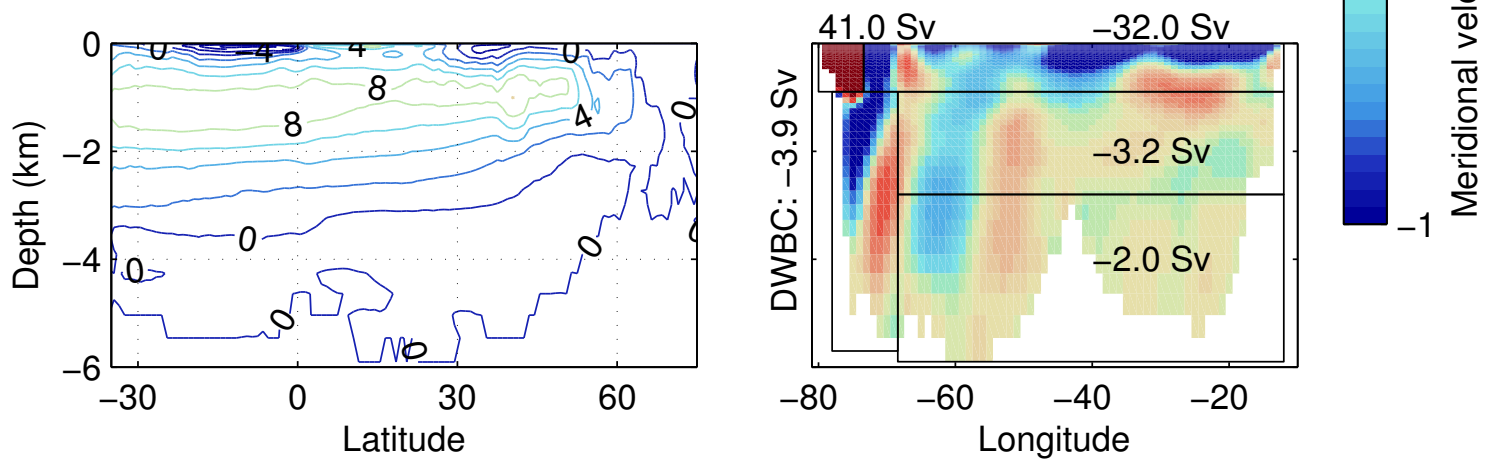

Figure 4-16: The LGM_Upper AMOC (bottom left) is weaker and shallower than the OCCA AMOC (top left); contour lines show zonally integrated transports in Sv. LGM_Upper has an enhanced wind-driven surface circulation with 41 Sv in the Gulf Stream and $31 \mathrm{~Sv}$ of surface return flow (bottom right) as compared to OCCA with transports of $35 \mathrm{~Sv}$ and $19 \mathrm{~Sv}$, respectively (top right). The weaker LGM_Upper AMOC is especially associated with the increased surface return flow. 


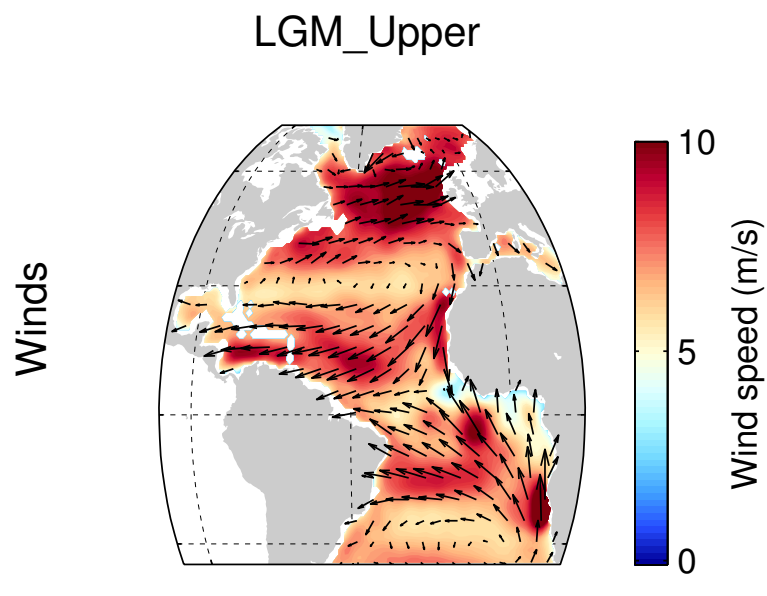

\section{LGM Upper - NCEP}
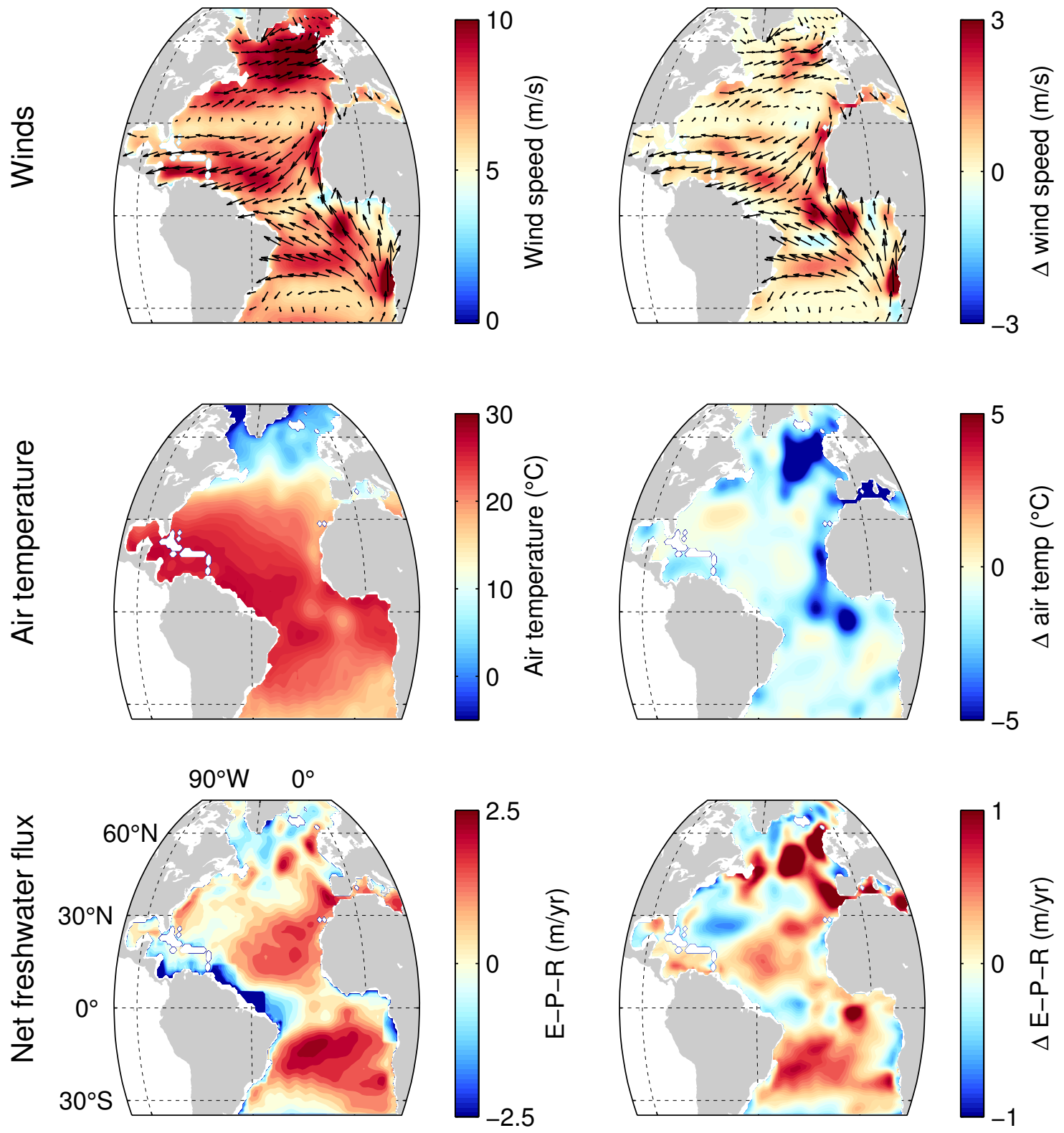

Figure 4-17: Annual mean adjusted atmospheric forcing for LGM_Upper (left column) and the difference with the Modern_TS estimate (right column). Wind speed and wind speed anomaly (top row) are shown in colors; vectors in both top row plots show the LGM_Upper wind direction. The middle row shows air temperature and anomaly. The bottom left plot shows net freshwater flux out of the ocean (evaporation - precipitation - runoff); positive values indicate net evaporation. In the bottom right plot, positive values indicate that, relative to Modern_TS, LGM_Upper has more evaporation and/or less runoff and precipitation (i.e. that the inferred LGM climate is drier). 
net evaporation in the subtropical gyres and net freshwater input near the Amazon and in the subpolar gyre. The plot of LGM-modern anomalies (Figure 4-17, bottom right) shows that LGM_Upper has increased subtropical gyre evaporation and a weakening of freshwater input in the subpolar gyre.

\subsection{Comparison to PMIP2 models}

The state estimates presented in this chapter are data-constrained but do not incorporate atmospheric dynamics. The PMIP2 simulations (see Section 2.3.1 for background) include a full dynamic atmosphere and ocean, but are not data-constrained. Much can be learned by comparing the results of the two methods; here we focus on misfit of modeled NSSTs to MARGO, changes in seasonality of NSSTs at the LGM, differences in the modeled wind fields, and comparison of the modeled sea ice distributions to sea ice proxy records. The results of five LGM PMIP2 simulations are considered here: CCSM3, MIROC 3.2, HAD CM3, FGOALS, and IPSL CM4 (see Table 2.2 for details). All PMIP2 analyses in this thesis were performed using version May-4-2012 of the database at http://pmip2.lsce.ipsl.fr/.

\subsubsection{Near sea surface temperatures}

Annual mean near sea surface temperatures (NSST) fields are shown in Figure 4-18 for LGM_AllSSTs and for five PMIP2 models. The PMIP2 models differ widely in their simulated LGM NSST patterns, both in terms of the magnitude and spatial patterns of LGM cooling. Misfits between PMIP2 and annual mean MARGO foraminiferal assemblagebased NSSTs (Figure 4-19) show that the PMIP2 models generally have larger misfits and stronger regional patterns than LGM_Upper does. This figure highlights known issues with the PMIP2 models in low latitudes: (1) these models have difficulty reproducing equatorial sea surface temperatures in the modern and in the LGM, and (2) the increased east-west temperature gradients seen in the MARGO NSST records are not reproduced in the PMIP2 models. Of all six models, only CCSM3 and LGM_Upper reproduce the very cold temper- 

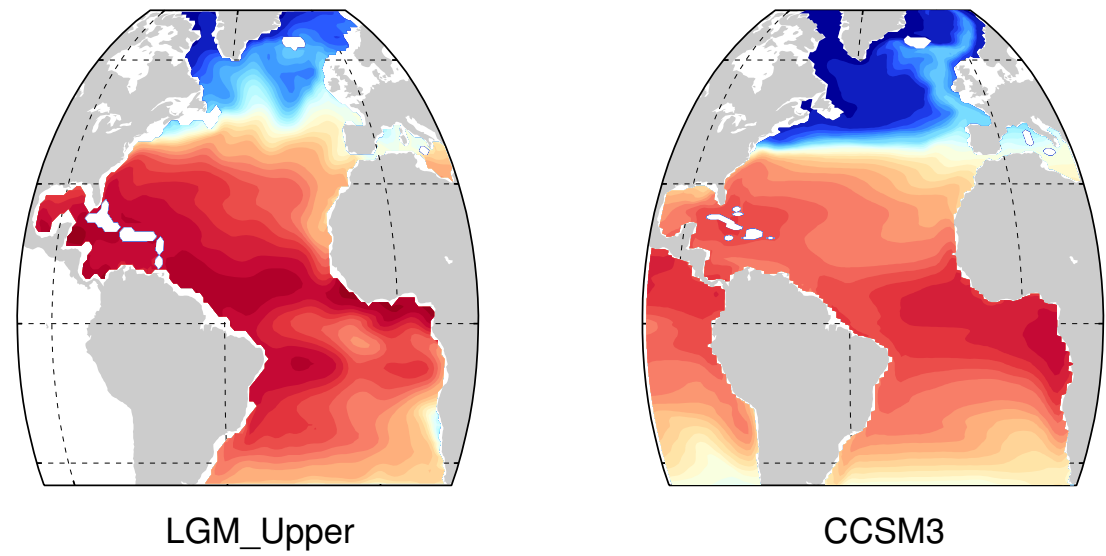

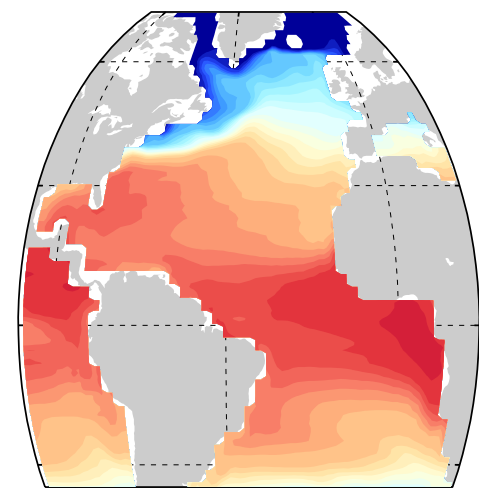

MIROC 3.2

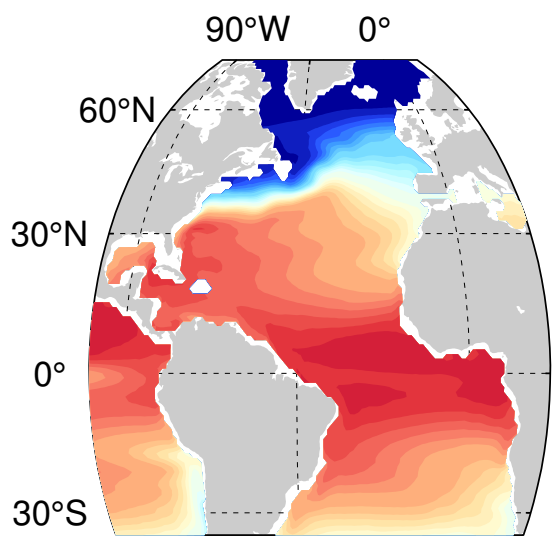

FGOALS
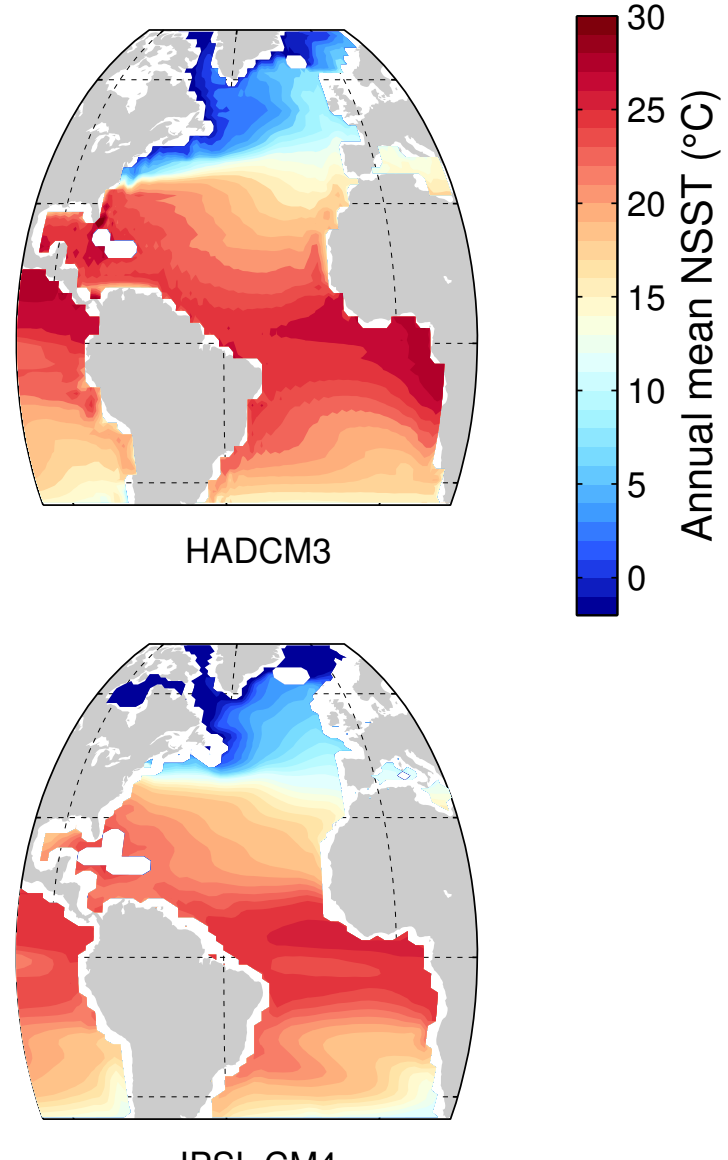

IPSL CM4

Figure 4-18: Annual mean near sea surface temperatures (NSSTs) for LGM_Upper and for five PMIP2 LGM simulations. 

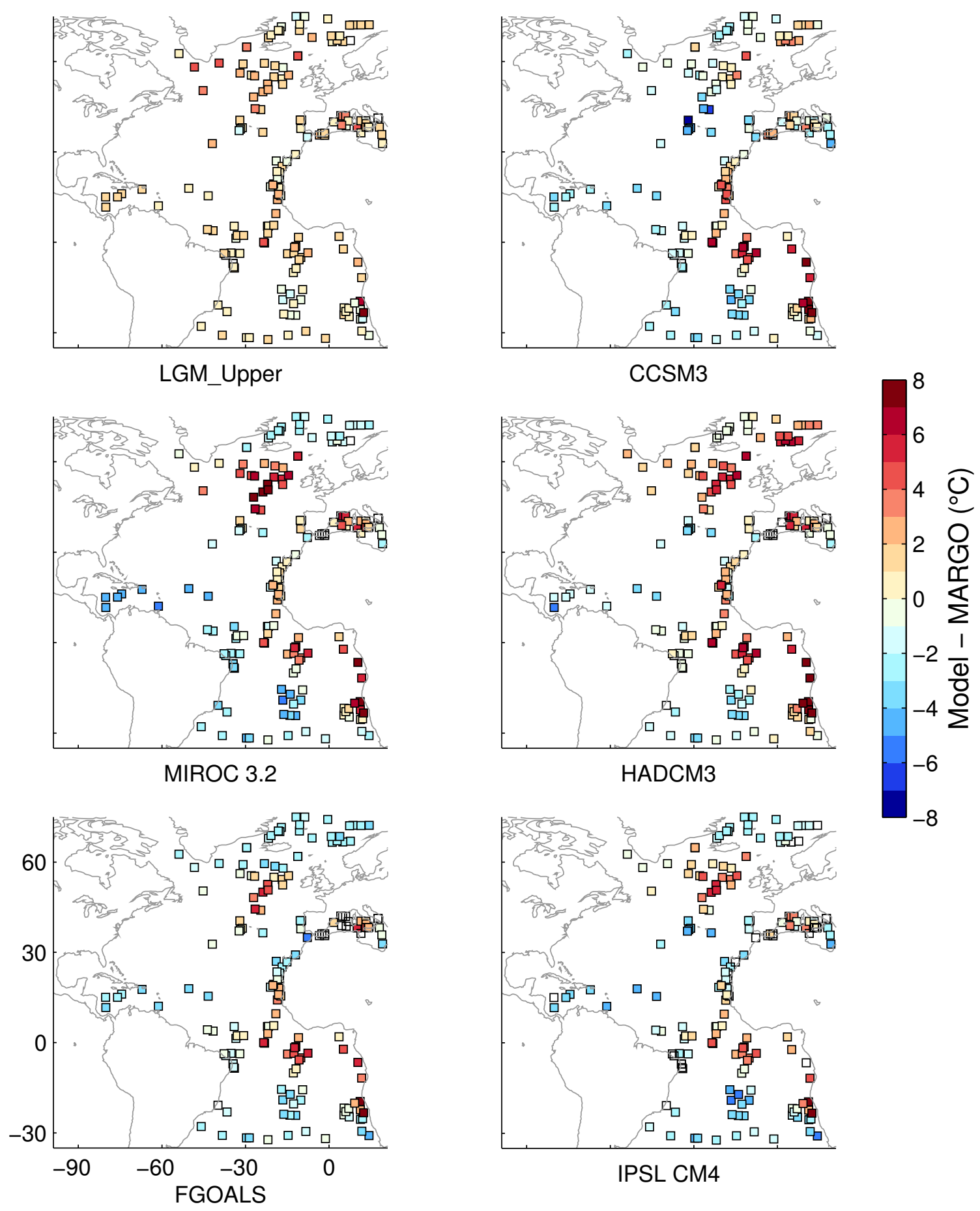

Figure 4-19: Misfit of annual mean NSSTs for LGM_Upper and five PMIP2 models to foram assemblage-based NSSTs from MARGO. Misfits of LGM_Upper are generally smaller than those of the PMIP2 models. 
atures seen in the foraminifera data southwest of Iceland in the subpolar gyre.

Cost functions are a convenient way to summarize the fit of models to data and will now be applied to analyze model-data fit for PMIP2; it should be remembered that since these models are not data-constrained, they are not expected to achieve the same level of fit to data as a data-constrained model would. The $J_{\text {data }}$ cost function used for LGM_AllSSTs is applied here (see Equation 4.1); this cost function is appropriate for analysis of the PMIP2 models as does not incorporate any of our biases - it uses the original, MARGO-reported data uncertainties. Figure 4-20 compares the mean cost for the fit of each PMIP2 model to each proxy type in each season. Costs are also shown for LGM_Upper using the same MARGO-reported data uncertainties; costs are relatively high for the alkenone and dinocyst data since the cost function used to compute these costs is not the cost function that was used to constrain the LGM_Upper estimate.

In terms of mean cost against all records (the bottom-most plot in Figure 4-20), the LGM_Upper estimate has a better fit to the data than the PMIP2 models do. Amongst the PMIP2 models, the IPSL CM4 model has a somewhat better fit than the other PMIP2 models for annual mean and summer mean temperatures; the FGOALS model has a somewhat better fit than the other PMIP2 models for winter mean temperatures. In terms of mean costs against each individual dataset (the upper four plots in Figure 4-20), different models perform particularly well against different individual datasets; this is because the datasets disagree with each other in some regions so that a model with warmer temperatures may agree better with one dataset, while a model with colder temperatures may agree better with a different dataset.

The high costs shown Figure 4-20 indicate that the PMIP2 models are not consistent with the MARGO data and their assigned uncertainties. This finding is in contrast to the results of Hargreaves et al. [2011], who applied ensemble evaluation techniques to find that the PMIP2 model ensemble is a reliable estimate of the MARGO NSST data (see Section 2.4.1 for details on their approach). Hargreaves et al. [2011] used the gridded MARGO NSST product described in Waelbroeck et al. [2009], whereas the individual proxy records 

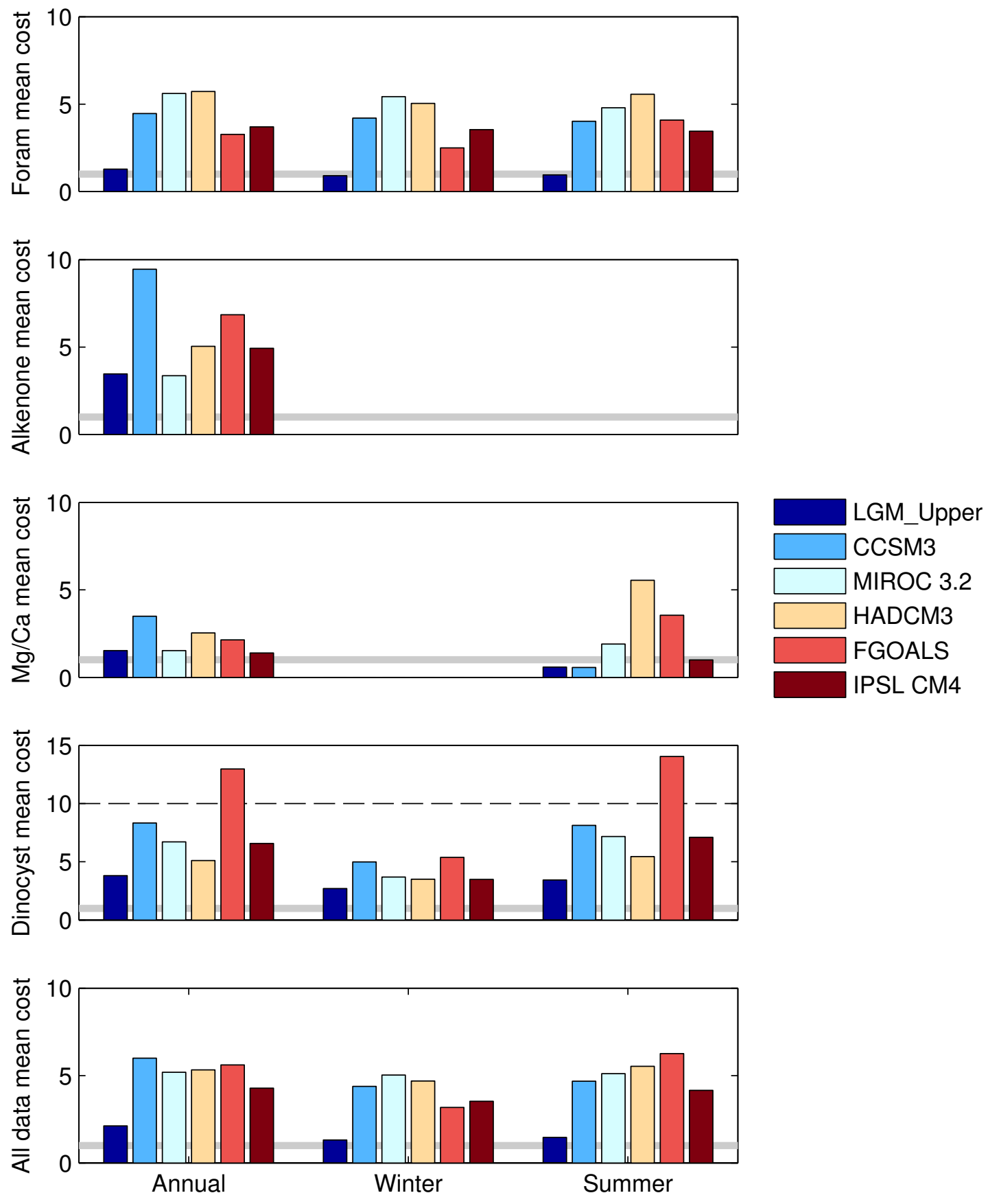

Figure 4-20: Mean cost for misfit of LGM_Upper and PMIP2 models to MARGO proxy records; the bottom plot gives the mean over all data. Uncertainties are unmodified MARGO-published uncertainties. Note that the dinocyst subplot has a different axis limit. The gray lines mark a mean cost of 1 , a value that indicates consistency of the model with the data. With MARGO-reported uncertainties, none of the models are consistent with the MARGO records, although LGM_Upper comes closest. 
are used here. The gridded product is heavily processed: it represents a compromise between the individual MARGO records, and is computed at $5^{\circ} \times 5^{\circ}$. By creating a smoothed, averaged field, Waelbroeck et al. [2009] have removed much of the signal recorded by the original proxy records.

\subsubsection{Seasonality}

Figure 4-21 shows the difference between mean August and February NSSTs in LGM_Upper and in the PMIP2 LGM models. Dark red colors in this figure mean the modeled northern hemisphere LGM summer is much warmer than the modeled northern hemisphere LGM winter; dark blue indicates the same for the southern hemisphere. In general, the six estimates show strong seasonality in the Gulf Stream region, in the Mediterranean Sea, and near $30^{\circ} \mathrm{S}$. LGM_Upper also shows relatively strong seasonality throughout the subpolar gyre and in the eastern low latitudes.

\subsubsection{Wind field}

Figure 4-22 shows annual mean wind fields $10 \mathrm{~m}$ above the surface for the PMIP2 LGM models and for LGM_Upper. The PMIP2 models all show similar LGM wind field patterns in the tropics and low latitudes: the equator is marked by relatively weak winds, while the easterlies are relatively strong, particularly in the northern hemisphere. The easterlies are also evident in the LGM_Upper wind field, though the northern and southern hemisphere easterlies are more similar in strength for LGM_Upper than they are for the PMIP2 models. LGM_Upper is also marked by more small-scale patterns in the low-latitude wind field than are the PMIP2 models. The westerly winds are stronger in the LGM_Upper estimate than they are in any of the other PMIP2 simulations. North of about $50^{\circ} \mathrm{N}$, the six models vary significantly in their estimated LGM wind fields; given the relatively coarse resolutions used in the PMIP2 models (see Table 2.2), this region may not be well-resolved.

Differences between modeled LGM and modern wind fields are shown in Figure 4-23, 

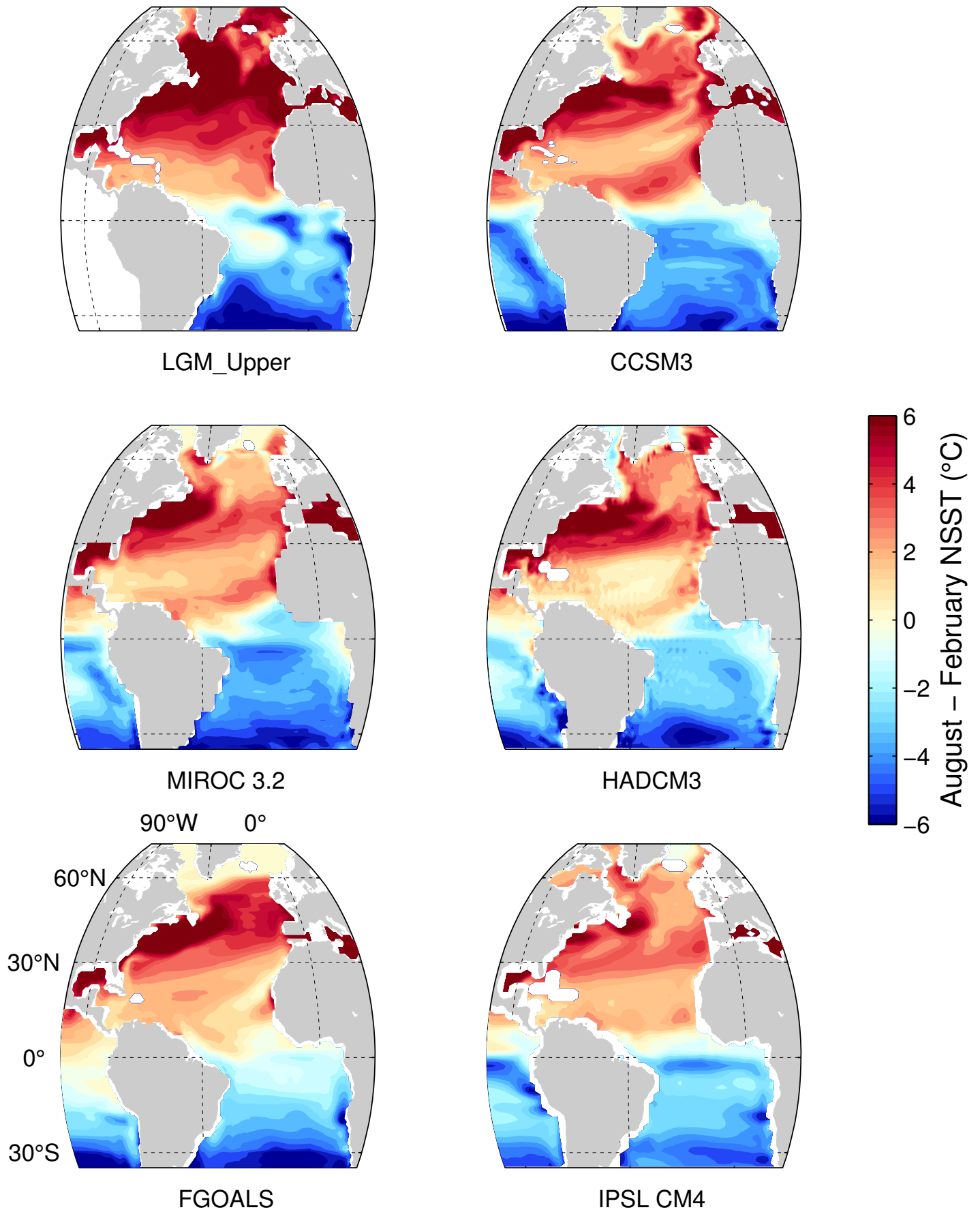

Figure 4-21: Difference between August and February NSSTs for LGM_Upper and five PMIP2 LGM simulations. 

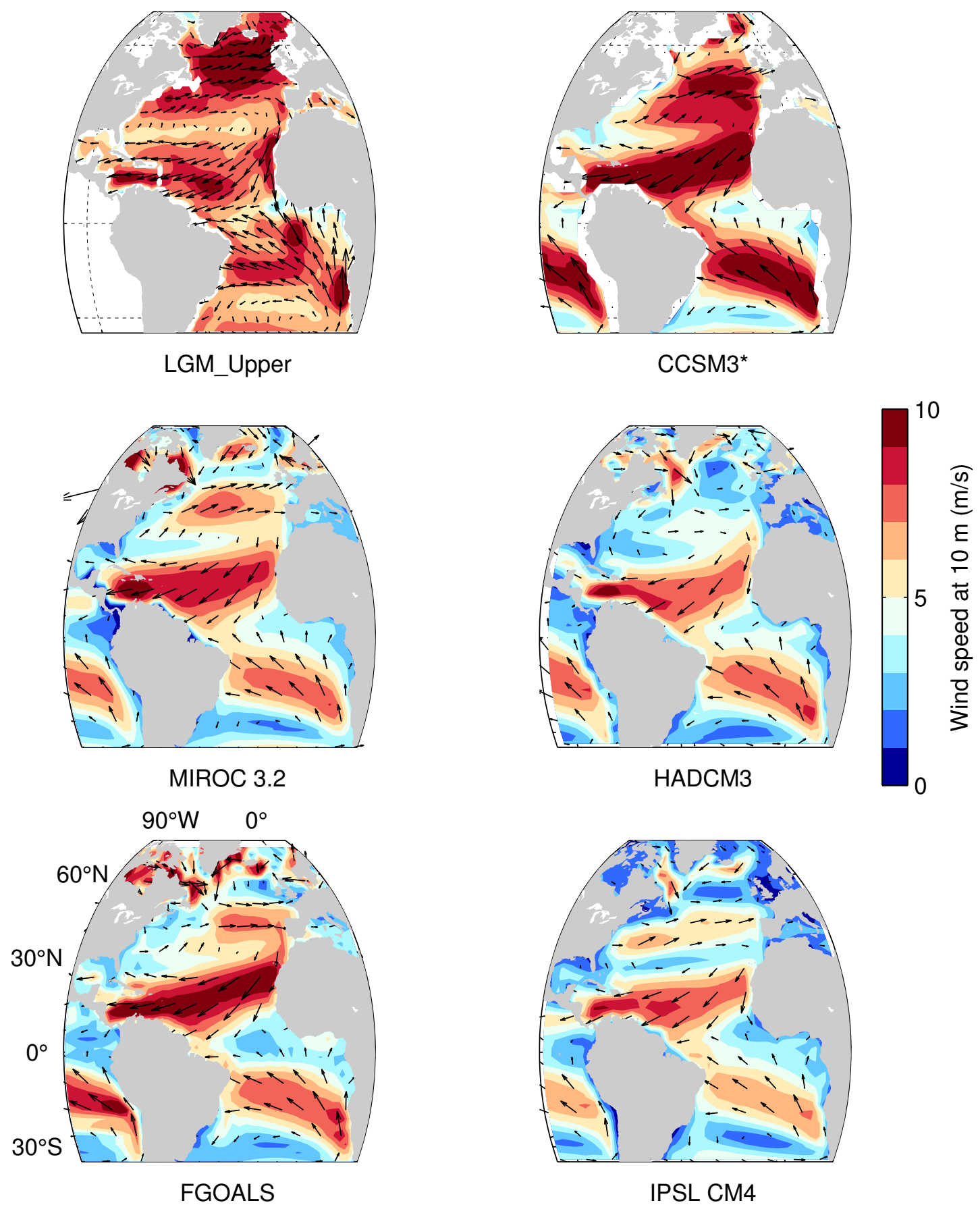

Figure 4-22: Annual mean winds for LGM_Upper and the PMIP2 LGM simulations. Colors show wind speed and arrows show direction. No model output was available for $10 \mathrm{~m}$ wind for CCSM3; the wind speed at the lowest level of the model is plotted instead. 

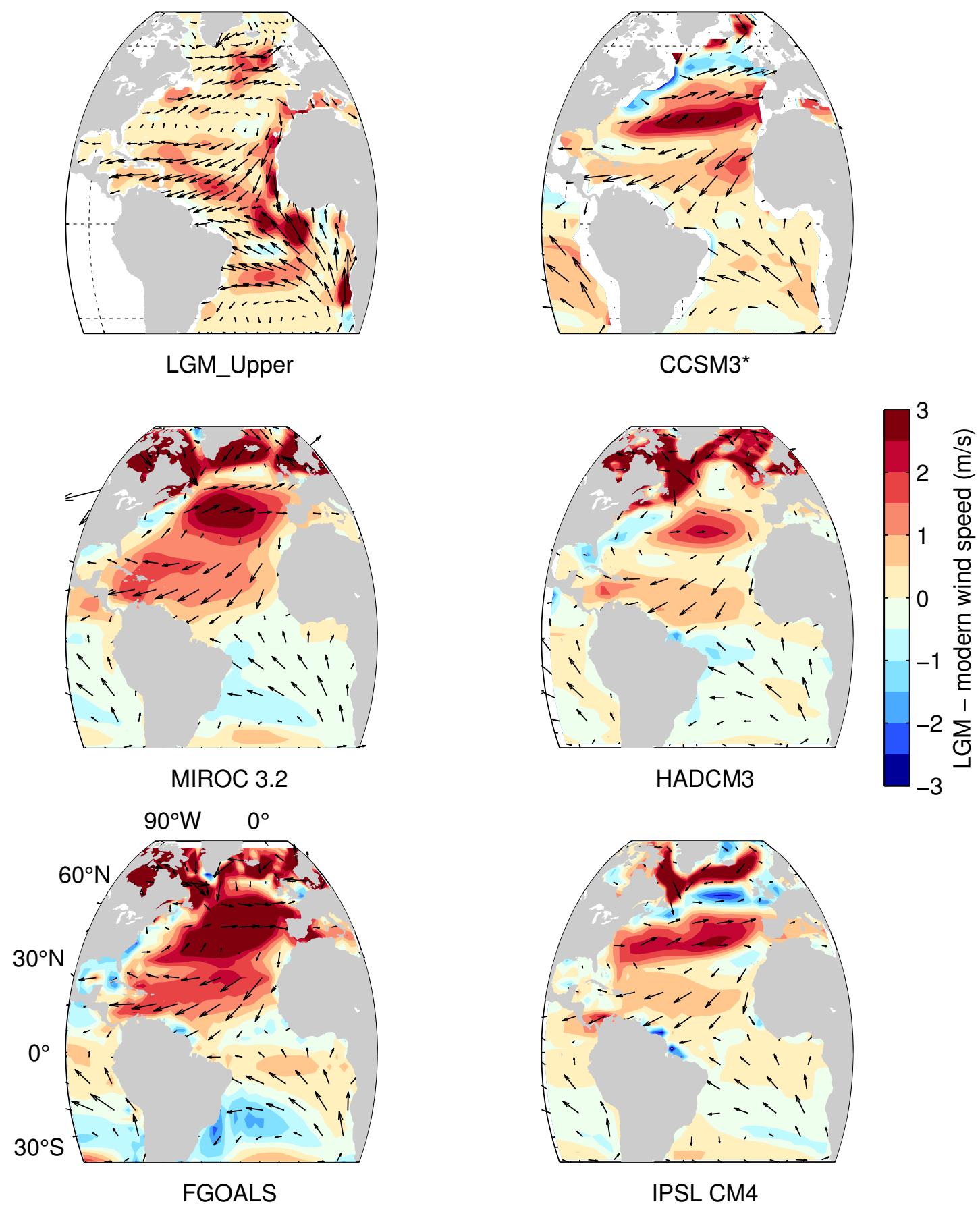

Figure 4-23: Differences between the LGM_Upper and Modern_TS wind fields (upper left) and between the LGM and pre-industrial PMIP2 simulations (all other plots); annual mean fields are used to compute differences. Colors show differences in wind speed, vectors show the modeled LGM wind field. 
which shows LGM_Upper - Modern_TS for the LGM_Upper estimate, and LGM - preindustrial (PI) for the PMIP2 simulations; the PI simulations will be referred to as modern in the following discussion (under the assumption that LGM to modern changes are likely much larger than PI to modern differences). All six estimates show significantly more regions with a stronger LGM wind field than with a weaker LGM wind field. LGM_Upper changes are strongest in the eastern low latitude basins, while the PMIP2 models show the strongest changes in the westerly wind belt and in the high latitudes north of about $50^{\circ} \mathrm{N}$. All of the models, including LGM_Upper, show strengthening of the westerlies at the LGM; the CCSM3 and IPSL anomalies also show evidence for a southward shift of the westerlies (i.e. the increased westerly wind strength region is accompanied by a band of decreased wind strength to the north in these two models).

Based on this discussion, it is not possible to conclude that any of these modeled wind fields is better than the others. However, it seems probable that the PMIP2 models have better estimates of the shifts in the westerly winds: the presence of the Laurentide ice sheet surely impacts westerly winds over the Atlantic Ocean, and these models simulate that impact directly. It would be interesting to apply the LGM_Upper configuration with larger prior wind field uncertainties in and near the westerly wind belt. On the other hand, in the low latitudes, the PMIP2 models show serious misfits with the MARGO NSST records (see Figure 4-19). Given that the LGM_Upper estimate has a much better fit to the proxies in this region, perhaps the LGM_Upper wind field shifts seen in this region are more indicative of actual wind field changes at the LGM than are the estimates of the PMIP2 models. It will be interesting to see if the fit of coupled models to the MARGO NSST data improves in the low latitudes with improvements in model resolution and model physics.

\subsubsection{Sea ice distributions}

Figures 4-24 and 4-25 show the winter and summer sea ice fraction in LGM_Upper and in four PMIP2 models (note that sea ice extent was not available for the IPSL model). Overlaid on the model fields are foraminifera and dinocyst proxy estimates of sea ice presence; 
these estimates are the same as shown in Figure 4-14 (see 4.4.3 for explanation of how MARGO sea ice estimates are mapped). Consider first the winter sea ice maps (Figure 424). The two models with moderate sea ice cover, CCSM3 and HadCM3M2, appear most consistent with the foraminifera data. On the other hand, the two models with the most limited sea ice cover, MIROC3.2 and LGM_Upper, appear most consistent with the dinocyst data. The disagreement between the two proxies is most apparent in the GIN seas. For summer sea ice distribution (Figure 4-25), FGOALS-1.0g clearly has far too much summer sea ice. Amongst the other models, LGM_Upper and CCMS3 probably have the best fit to the dinocyst-based sea ice estimates, while MIROC3.2 and HADCM3M2 probably have the best fit to the foram-based sea ice estimates. See page 172 for a discussion of why sea ice distribution may be too limited in LGM_Upper.

Although we have not defined uncertainty estimates for the sea ice proxies, they are likely to be large. Additionally, the foram-based and dinocyst-based sea ice estimates disagree, especially in winter. Given these issues, the data appear to be insufficient to reject any of the model estimates except perhaps FGOALS-1.0g.

\subsection{Chapter summary}

This chapter presents ocean state estimates for the LGM - dynamical reconstructions that account for proxy records of LGM ocean state, equations of oceanic motion, uncertainties in the data, and uncertainties in the model boundary and initial conditions. The focus has been on reconstructing upper ocean properties based on MARGO near sea surface temperature proxies. Novel contributions of this chapter include the following.

- Previous applications of inverse methods to other proxy types such as LGM records of $\delta^{13} \mathrm{C}$ have used low-resolution models with simplified dynamics. It is now possible to use a full GCM at a moderate resolution of $1^{\circ}$.

- Inverse methods have not previously been applied to LGM near sea surface temperature data. It has been demonstrated here that it is feasible to do so, and that the 

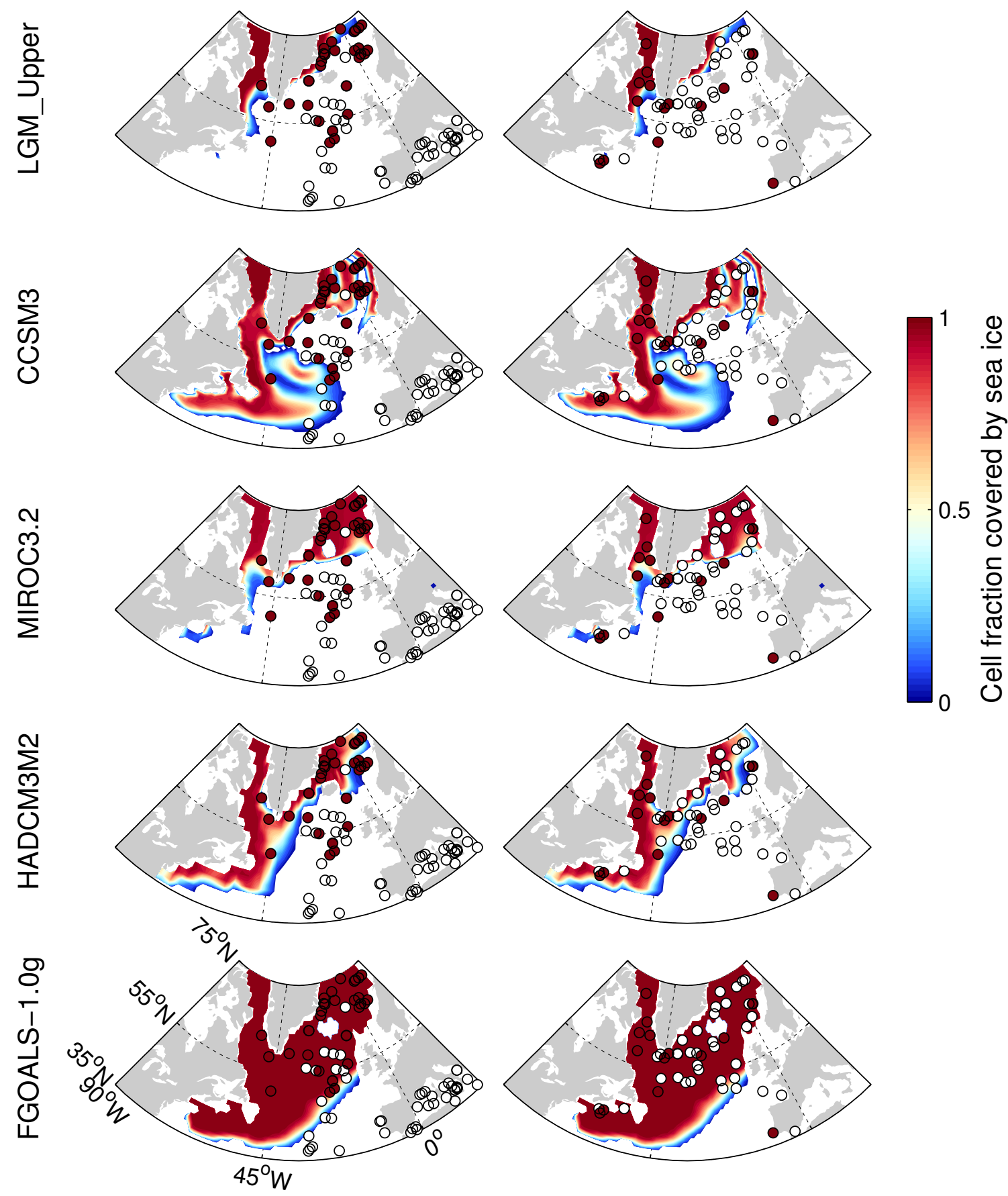

Figure 4-24: Winter sea ice distributions in four PMIP2 models and in LGM_Upper. Subplots of each row show the same model field with an overlay of foraminifera (left) or dinocyst (right) data. Model fields are cell fraction covered by sea ice (fractions below 0.02 not shown); for proxies, red circles indicate sea ice presence, empty circles indicate sea ice absence. 

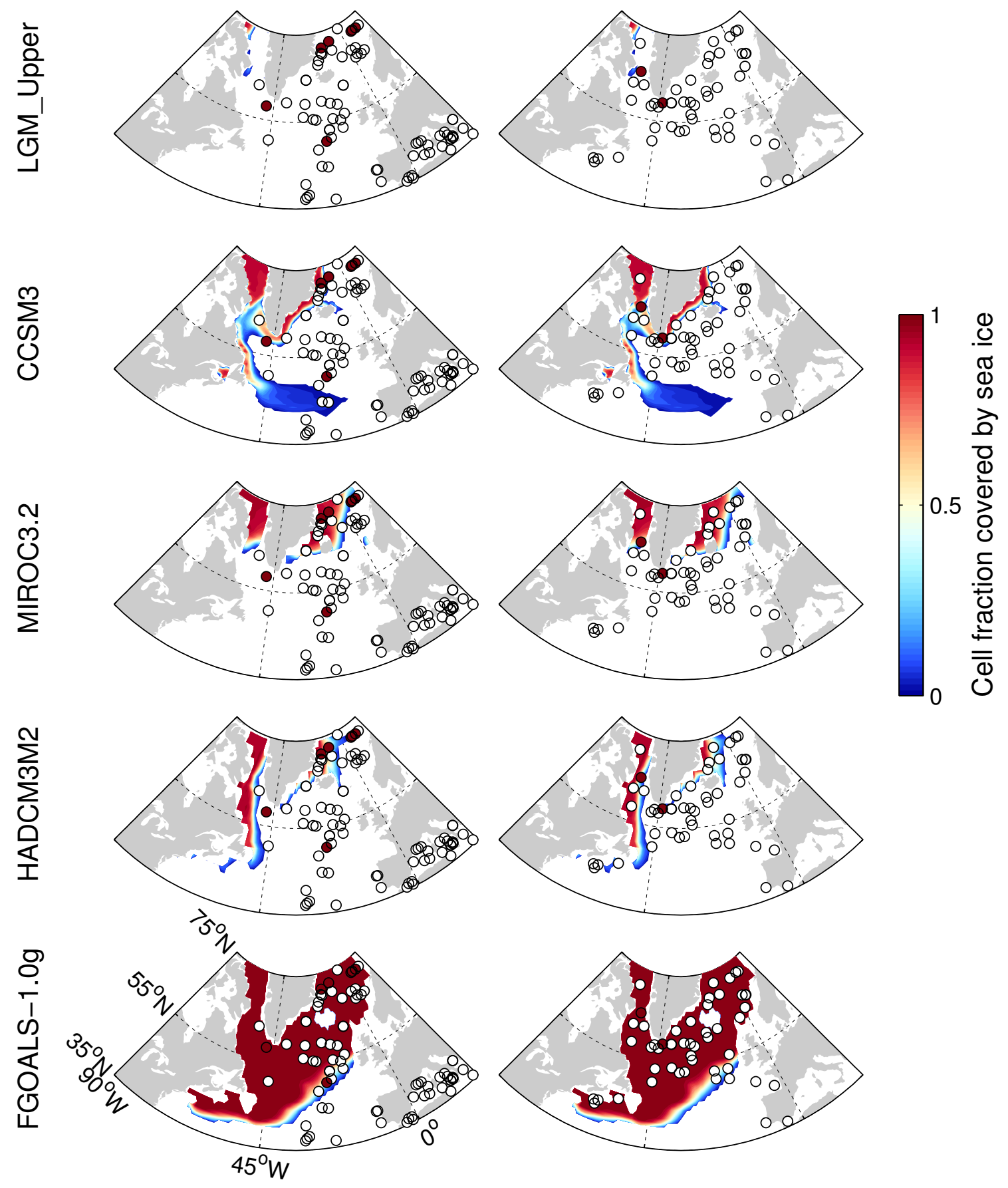

Figure 4-25: Summer sea ice distributions in four PMIP2 models and in LGM_Upper. Subplots of each row show the same model field with an overlay of foraminifera (left) or dinocyst (right) data. Model fields are cell fraction covered by sea ice (fractions below 0.02 not shown); for proxies, red circles indicate sea ice presence, empty circles indicate sea ice absence. 
inferred ocean circulation and LGM atmospheric forcing fields are plausible.

- A best estimate of upper ocean conditions at the LGM has been presented. This solution, called LGM_Upper, demonstrates that it is possible to find an estimate of ocean state that is consistent with the MARGO NSST records, if published uncertainties are increased for dinoflagellate cyst and alkenone data north of $40^{\circ} \mathrm{N}$.

- It has been shown that the PMIP2 models are not consistent with the MARGO NSST records and their original published uncertainties, in disagreement with the Hargreaves et al. [2011] comparison of the PMIP2 models against the gridded MARGO NSST product. As expected, the LGM_Upper estimate shows better agreement with the MARGO data and their uncertainties than any of the PMIP2 models do.

- The LGM_Upper and PMIP2 sea ice distributions have been compared to sea ice proxy data for the LGM. Given large uncertainties in the proxy data, LGM_Upper and three of the four examined PMIP2 models show plausible sea ice distributions.

Open questions remain. On the modeling side, the AMOC is weak (due to increased surface return flow) in 10-year estimates of the modern circulation using this state estimation framework (see Section 3.5), complicating interpretation of the estimated LGM transports and MOC. Time has not permitted development of state estimates with different first guess conditions here, so it is not known how sensitive the results are to the first guess initial conditions, atmospheric forcing, or southern boundary conditions. Evaluation of these sensitivities is an important are for future research, as is an evaluation of the sensitivity of the solution to prior uncertainty estimates.

On the proxy side, the MARGO NSST data are sparse with poorly known uncertainties. Also, disagreement between different proxy types in the northern North Atlantic is significantly larger than would be consistent with the uncertainties typically assigned to the data. There is a general need for proxy records with a more complete geographic distribution, and for proxy records of other aspects of ocean state (temperatures throughout the thermocline, salinity, sea ice, surface currents, etc). Many upper ocean proxy records exist that 
have not been included in the state estimates presented here; thus, progress can be made both through incorporating additional records and proxy types in ocean state estimates, as well as through development of new proxies and generation of new records. These many open questions are fruitful topics for future investigation; for the moment, it suffices to say that they should be kept in mind in interpreting the results presented herein. 


\section{Chapter 5}

\section{Deep ocean conditions at the Last Glacial Maximum}

This chapter explores deep ocean conditions at the LGM, with a focus on water mass pathways and distributions. The upper ocean and deep ocean are clearly not independent; they are connected in many ways, including via the meridional overturning circulation and total mass and salt conservation. However, estimation of LGM deep ocean conditions requires different proxy records and longer simulations than estimation of upper ocean conditions; the topics are thus treated in separate chapters.

Ocean state estimates presented in this chapter incorporate the benthic $\delta^{13} \mathrm{C}_{\text {calcite }}$ and $\delta^{18} \mathrm{O}_{\text {calcite }}$ compilation of Marchal and Curry [2008], in addition to the MARGO near sea surface temperature records used in Chapter 4. This chapter builds on previous parts of the thesis, particularly Section 3.4 for model treatment of water column isotopes, Section 3.5 for approaches to extending run length for equilibrium ocean estimation (EOE), and Chapter 4 for modeling the LGM and incorporating MARGO data.

State estimates 10 years in length were used to reconstruct upper ocean conditions in Chapter 4. As was shown in Section 3.5, 10 years is too short to constrain deep ocean tracer fields with sparse data - the connection between surface forcing and deep ocean conditions is too limited on this timescale. In Section 3.5, a framework for EOE was presented to 
address this problem. Asynchronous timestepping and carry-over control were shown to be effective means of extending the length of ocean state estimates. Since a number of significant problems were noted with current capabilities in EOE, the state estimates presented in this chapter should be considered exploratory. Nevertheless, these results represent the current state of the art in estimation of paleo ocean conditions, and they provide many indications of how to proceed in this research area in the future.

This chapter is organized as follows. The data used in this chapter and their uncertainty assignments are described in Section 5.1. Model extensions are needed to permit inclusion of these data as constraints in our state estimation approach; these extensions are described in Section 5.2. Section 5.3 describes the process of obtaining a longer state estimate, and compares the overall model-data fit for a shorter and longer state estimate. Properties of these estimates are analyzed in Section 5.4, and our results are compared with those of other models in Section 5.5. Section 5.6 concludes the chapter.

\section{1 $\quad \delta^{18} \mathbf{O}$ and $\delta^{13} \mathbf{C}$ data from benthic foraminifera}

The Marchal and Curry [2008] (hereafter MC08) compilation of $\delta^{18} \mathrm{O}_{\text {calcite }}$ and $\delta^{13} \mathrm{C}_{\text {calcite }}$ measured on samples of LGM age are used in this chapter to constrain water mass distributions and pathways in ocean state estimates. This compilation was described in Section 2.2.3, and Figure 2-6 showed both datasets, as well as the distribution of core depths for the records. The MC08 data are interpreted here as recording mean LGM conditions, similar to the assumption of steady state conditions used by Marchal and Curry [2008]. Benthic foraminifera depend on the rain of organic matter from the surface ocean, and are therefore likely to exhibit seasonality in growth rates and species assemblages (see, e.g., Duchemin et al. 2008). However, the majority of the MC08 data fall in deeper ocean waters where seasonal dependence of water isotopes and temperatures is presumably limited. Thus, even if benthic organisms themselves have a preferential growing season, the conditions recorded in their shells should be similar to longer-term mean conditions. As is 
the case for all proxy records, uncertainty estimates are difficult to make for the MC08 data (see Section 2.2.3 for a full discussion). An uncertainty of $0.2 \%$ is used on all MC08 records. This uncertainty is in keeping with conservative uncertainties assumed by MC08; although MC08 also experimented with an uncertainty of $0.1 \%$ for the $\delta^{13} \mathrm{C}_{\text {calcite }}$ records, only the larger uncertainty is used here because of the large uncertainties associated with the effect of remineralization on $\delta^{13} \mathrm{C}_{\text {calcite }}$ (see Section 3.4.2).

To compare model and data, a mapping of the gridded model field onto proxy record locations is required; this mapping is more difficult for benthic proxies than for planktonic ones. Benthic proxies are measurements of the shells of organisms that live near the sediment/water interface, but the location of that interface is poorly resolved in the model: the model has $1^{\circ} \mathrm{x} 1^{\circ}$ resolution in the horizontal, has grid cell thicknesses of up to $450 \mathrm{~m}$, and uses an approximate, smoothed bathymetry. If a naive mapping is used, a large fraction of benthic proxy records are not associated with any model grid cells because those sediment core locations fall under the model's sediment-water interface. This issue is addressed here by reassigning model grid cells for comparison to these proxy records by hand. Most often, the reassignment uses a model grid cell that is shifted horizontally by one step from the naively mapped cell. Rarely, such a reassignment is not possible, and a model grid cell that is shifted upward by one vertical level is used instead.

\subsection{Forward model and state estimation configuration}

The state estimation configuration of LGM_Upper (Chapter 4) is used here, with the following adaptations. $\delta^{13} \mathrm{C}_{\text {DIC }}$ and $\delta^{18} \mathrm{O}_{\text {water }}$ are modeled following the approach described in Section 3.4. Model values of $\delta^{13} \mathrm{C}_{\text {calcite }}$ and $\delta^{18} \mathrm{O}_{\text {calcite }}$ are then estimated using the approach of MC08:

$$
\delta^{13} \mathrm{C}_{\text {calcite }}=0.13+0.90 \cdot \delta^{13} \mathrm{C}_{\text {DIC }}
$$




$$
\delta^{18} \mathrm{O}_{\text {calcite }}=3.35+0.97 \cdot \delta^{18} \mathrm{O}_{\text {water }}-0.21 \cdot T
$$

See Marchal and Curry [2008] (their Figures 1 and 2) for regression curves and accompanying discussions.

First guess initial and boundary conditions on $\delta^{13} \mathrm{C}_{\mathrm{DIC}}$ and $\delta^{18} \mathrm{O}_{\text {water }}$ are defined as follows. $\delta^{13} \mathrm{C}_{\mathrm{DIC}}$ initial conditions are set to $0.5 \%$ everywhere, concentration boundary conditions $\delta^{13} \mathrm{C}_{\mathrm{DIC} s f c}$ over the top $1000 \mathrm{~m}$ of the model are set to linearly increase with latitude as $\delta^{13} \mathrm{C}_{\mathrm{DIC} s f c}=0.93+0.013 \cdot$ lat, and southern boundary conditions $\delta^{13} \mathrm{C}_{\mathrm{DIC} s b c}$ are set to linearly increase with increasing depth (where depth is positive down) as $\delta^{13} \mathrm{C}_{\mathrm{DIC} s b c}=$ $0.88-0.00041 \cdot$ depth. These first guess surface and southern boundary conditions are linear approximations to the MC08 LGM $\delta^{13} \mathrm{C}_{\text {calcite }}$ distributions between 500 and $1500 \mathrm{~m}$ and between $30-45^{\circ} \mathrm{S}$, respectively. For $\delta^{18} \mathrm{O}_{\text {water }}$, first guess initial and boundary conditions are computed by adding a constant offset of $1 \%$ to the LeGrande and Schmidt [2006] gridded dataset of modern $\delta^{18} \mathrm{O}_{\text {water }}$. An offset of $1 \%$ is consistent with reconstructions of the change in mean ocean $\delta^{18} \mathrm{O}_{\text {water }}$ between today and the LGM [Duplessy et al., 2002].

The control variables are the same as described in Section 3.4, as is the form of the cost function contribution from the control variables $J_{c t r l}$. The first guess initial and boundary condition estimates of $\delta^{13} \mathrm{C}_{\text {calcite }}$ and $\delta^{18} \mathrm{O}_{\text {calcite }}$ are rough approximations; as such, control variable uncertainties for these conditions are set to a relatively large value of $0.4 \%$.

The model-data misfit cost $J_{\text {data }}$ is a sum of the MARGO contribution and the contribution from the isotopic records:

$$
J_{\text {data }}=J_{M A R G O}+J_{\delta^{13} \mathrm{C}_{\text {calcite }}}+J_{\delta^{18} \mathrm{O}_{\text {calcite }}}
$$


where $J_{M A R G O}$ is given in Equation 4.1 and the isotopic contributions are as follows.

$$
\begin{aligned}
& \left.J_{\delta^{13} C_{\text {calcite }}}=\frac{2}{n y} \sum_{y=n y / 2}^{n y} \overline{\left(\boldsymbol{\delta}^{\mathbf{1 3}} \mathbf{C}_{\text {calcite,mod }}(y)\right.}-\boldsymbol{\delta}^{\mathbf{1 3}} \mathbf{C}_{\text {calcite,obs }}\right)^{T} . \\
& \mathbf{W}_{\delta^{13} \mathbf{C}_{\text {calcite }}} \cdot\left(\overline{\boldsymbol{\delta}^{13} \mathbf{C}_{\text {calcite,mod }}(y)}-\boldsymbol{\delta}^{13} \mathbf{C}_{\text {calcite,obs }}\right) \\
& J_{\delta^{18} \mathbf{O}_{\text {calcite }}}=\frac{2}{n y} \sum_{y=n y / 2}^{n y}\left(\overline{\boldsymbol{\delta}^{\mathbf{1 8}} \mathbf{O}_{\text {calcite,mod }}(y)}-\boldsymbol{\delta}^{\mathbf{1 8}} \mathbf{O}_{\text {calcite,obs }}\right)^{T} . \\
& \mathbf{W}_{\boldsymbol{\delta}^{18} \mathbf{O}_{\text {calcite }}} \cdot\left(\overline{\boldsymbol{\delta}^{18} \mathbf{O}_{\text {calcite,mod }}(y)}-\boldsymbol{\delta}^{18} \mathbf{O}_{\text {calcite,obs }}\right)
\end{aligned}
$$

$\overline{\boldsymbol{\delta}^{\mathbf{1 3}} \mathbf{C}_{\text {calcite,mod }}(y)}$ and $\overline{\boldsymbol{\delta}^{\mathbf{1 8}} \mathbf{O}_{\text {calcite,mod }}(y)}$ are the annual mean modeled $\delta^{13} \mathrm{C}_{\text {calcite }}$ and $\delta^{18} \mathrm{O}_{\text {calcite }}$ for year $y, \delta^{13} \mathbf{C}_{\text {calcite,obs }}$ and $\delta^{18} \mathbf{O}_{\text {calcite,obs }}$ are the MC08 proxy observations, and $\mathbf{W}_{\delta^{13} \mathrm{C}_{\text {calcite }}}$ and $\mathbf{W}_{\delta^{18} \boldsymbol{O}_{\text {calcite }}}$ are the weighting matrices. The simplifying assumption is made that all data are independent, so that all off-diagonoal elements of $\mathbf{W}_{\delta^{18} \mathbf{O}_{\text {calcite }}}$ are zero.

\subsection{The search for an acceptable solution}

As a simple first test, the 10 year LGM_Upper estimate is extended by incorporating $\delta^{13} \mathrm{C}_{\text {calcite }}$ and $\delta^{18} \mathrm{O}_{\text {calcite }}$ data; the resulting estimate is referred to as "LGM_Deep10yr". As shown in Figure 5-1, it is possible to obtain a 10 year estimate with a satisfactory fit to the benthic isotope and MARGO NSST records. As discussed in Chapter 3 (see especially Figure 3-17), adjustments to tracer initial conditions are highly effective in improving the fit to deep tracer data in short state estimates. Thus, the good fit of LGM_Deep10yr to the MC08 data may be, to some extent, independent of whether the estimate's ocean circulation is a reasonable approximation of LGM ocean state.

The equilibrium ocean estimation (EOE) techniques of Section 3.5 are now applied to build a longer LGM state estimate. The goals are (1) to increase the connection between 
the imposed boundary conditions and the interior properties in the model and (2) to identify a seasonal steady state estimate of LGM ocean circulation. To extend the simulation length, the LGM_Deep10yr controls are applied to a 20-year run that is constrained by the same data sets; this process is continued through 40 and 80 -year estimates. At a run length of 80 years, the procedure is unable to obtain any improvement, so run length is not extended further. As elsewhere in the thesis, the cost function is applied to the last half of the estimate; the mid-point of this period for the 80 year run, year 60 , is used for analysis and is hereafter referred to as "LGM_Deep". As shown in Figure 5-2, results are encouraging. Although the fit to most proxies is somewhat degraded in LGM_Deep as compared to LGM_Deep10yr, the fit is still relatively good. In particular, the LGM_Deep solution still has better mean costs for MARGO data than the PMIP2 models do (to see this, compare the mean costs shown in Figure 5-2 to those shown in Figure 4-20).

\subsection{Analysis of LGM_Deep10yr and LGM_Deep}

\subsubsection{NSSTs and the MOC}

LGM_Deep has a degraded fit to the MARGO data as compared to LGM_Deep10yrbecause of model drift; although the state estimation approach applied here is generally effective at reducing drift in short runs (see Figure 3-18), drift clearly remains an issue in the longer LGM_Deep estimate. An analysis of how NSSTs differ in LGM_Deep10yr and LGM_Deep is useful for understanding where and how this drift affects the solution as run length is increased. Figure 5-3 shows LGM_Deep NSSTs and the anomaly against the LGM_Deep10yr estimate; the anomaly plots highlight regions of the model that suffer from strong model drift. Drift appears stronger in winter than in summer; this may explain an interesting aspect of Figure 5-2: that the fit of LGM_Deep to summer mean foram assemblage-based NSSTs is not as degraded as the fit to winter foram NSSTs. In all seasons, the region north of about $40^{\circ} \mathrm{N}$ is more impacted by model drift than other regions (Figure 5-3); wintertime drift is also seen in the upwelling region near $15^{\circ} \mathrm{S}$. These observations support the 

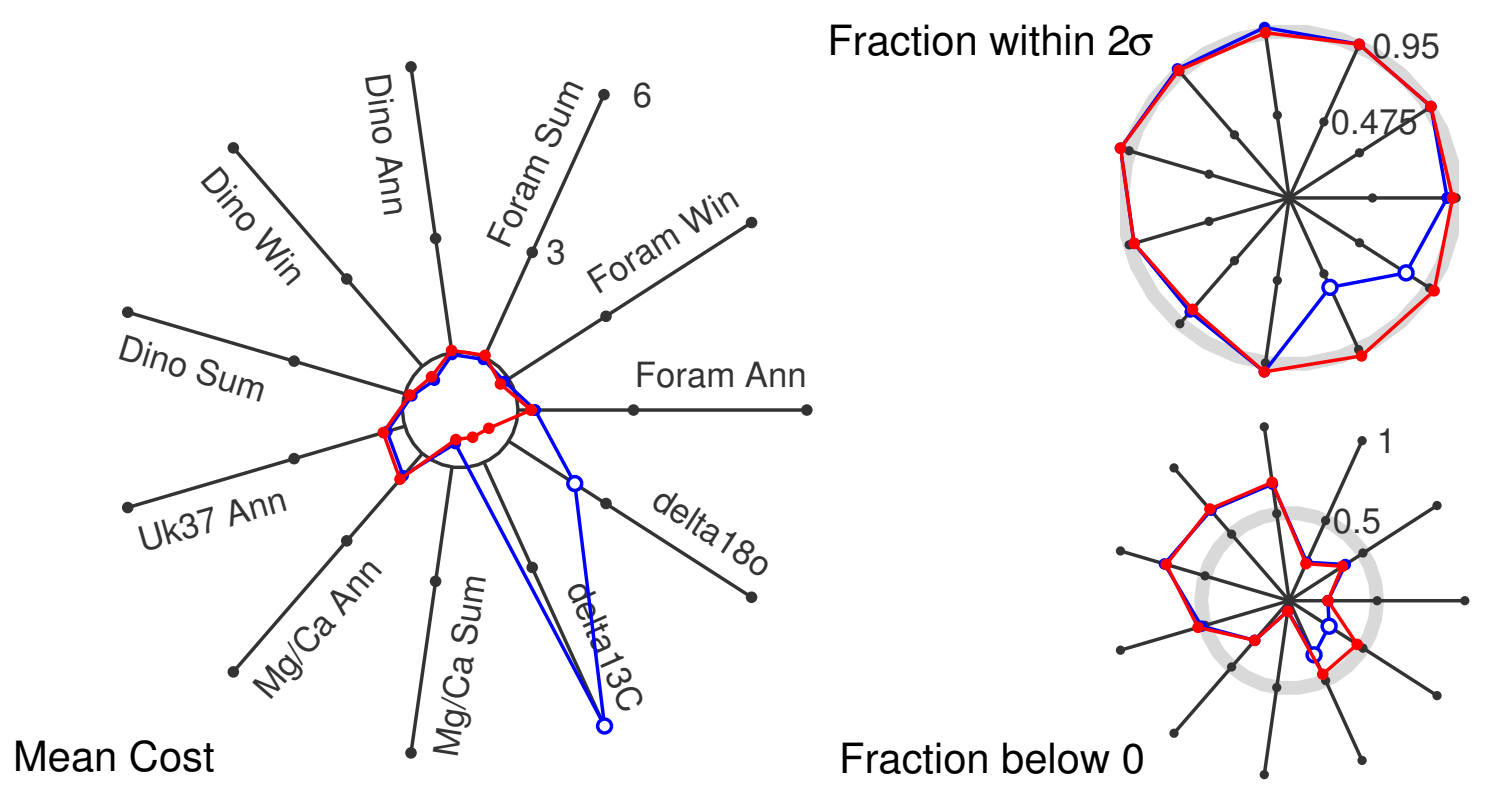

Figure 5-1: A comparison of two 10 year state estimates: LGM_Upper (blue), which is constrained by NSST proxies, and the LGM_Deep10yr (red), which is constrained by NSST and benthic isotope proxies. LGM_Deep10yr fits the benthic isotope data well while maintaining a good fit to the NSST proxies. Fit to excluded data (open circles) is diagnostic only.
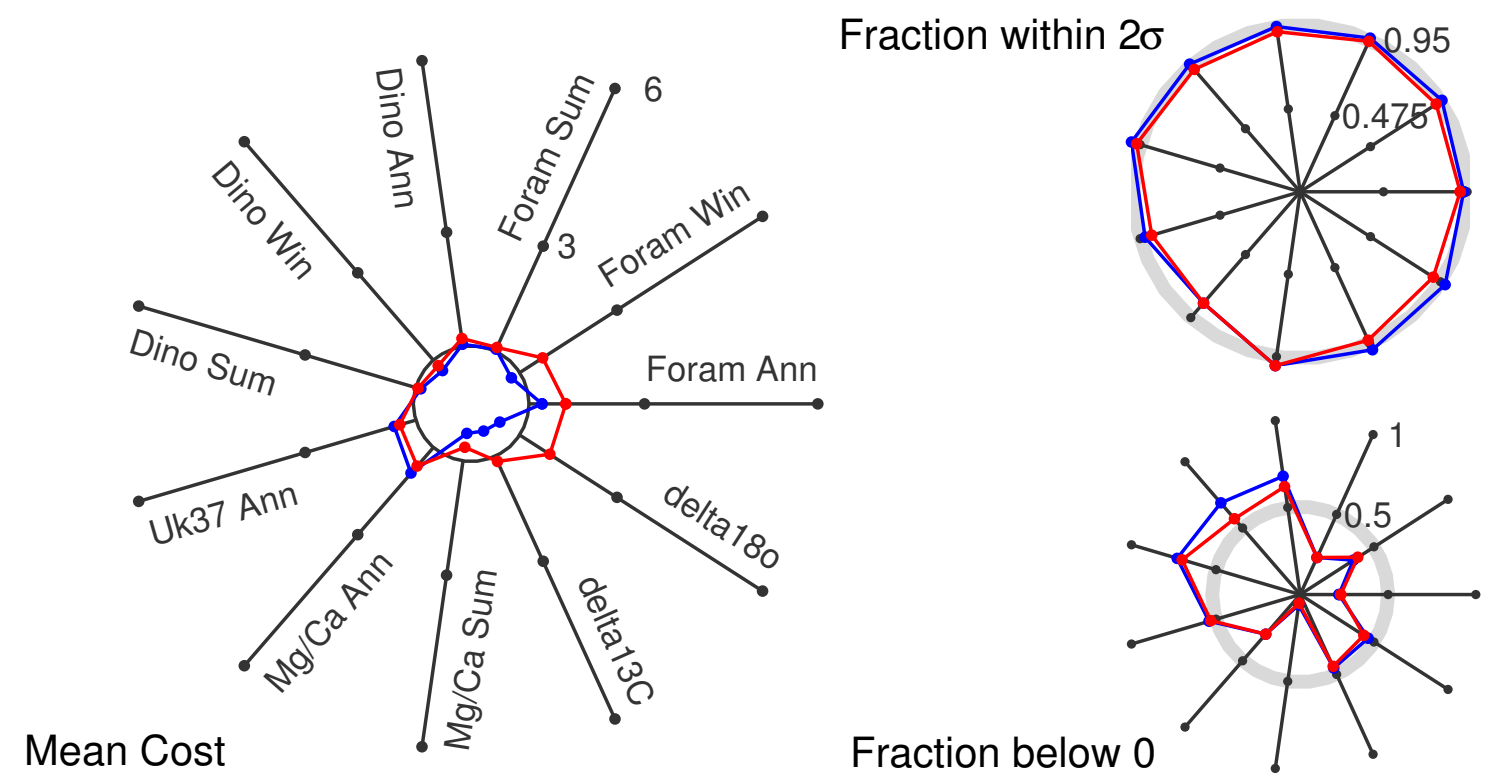

Figure 5-2: As run length is increased from 10 years (LGM_Deep10yr, blue) to 80 years (LGM_Deep, red), the fit to most proxies is somewhat degraded. However, the fit of the solution to the data is still relatively good, with mean costs around 1.5 and below. 

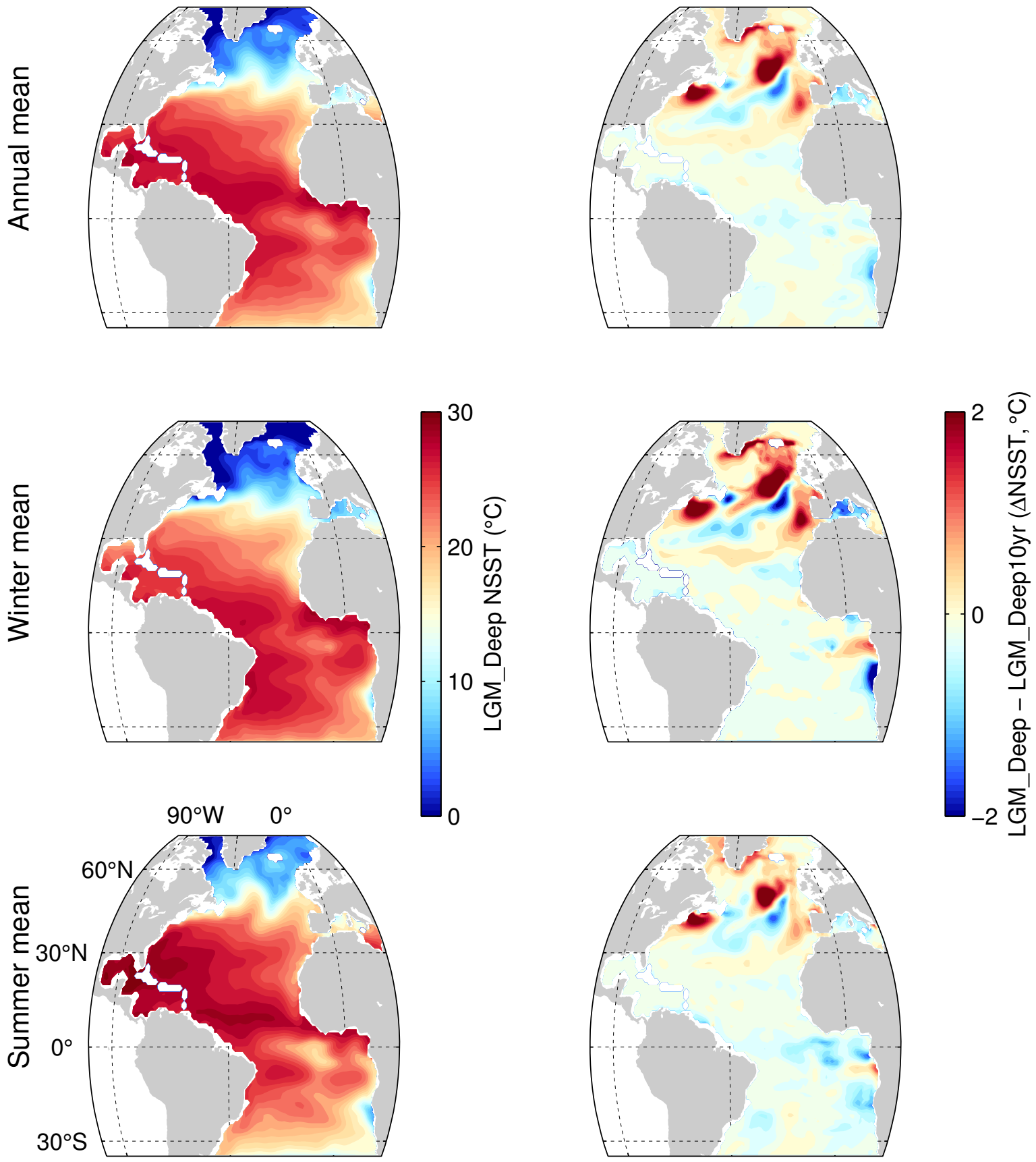

Figure 5-3: Annual, winter, and summer mean NSSTs for the LGM_Deep estimate (left), and the difference LGM_Deep - LGM_Deep10yr (right). Regions with large differences are regions that suffer from particularly severe model drift as run length is increased. 
hypothesis that model drift in deep ocean temperatures is influencing NSSTs in regions impacted by convection or upwelling. That these processes are most active in winter may explain why differences between LGM_Deep and LGM_Deep10yr are largest in winter. In working towards longer runs with the modern configuration (Section 3.5), maintenance of the properties of newly formed deep waters was a primary problem. Obtaining improved control over deep ocean properties will be key to future progress in equilibrium ocean estimation.

Figure 5-4 shows the MOC and $29^{\circ} \mathrm{N}$ transports in the OCCA, LGM_Deep10yr and LGM_Deep estimates. The LGM_Deep10yr transports are similar to those of the 10 year LGM_Upper solution presented in Chapter 4 (see Figure 4-16), indicating that incorporating the benthic isotope data has not substantially changed for state estimates 10 years in length. In contrast, LGM_Deep transports show strong increases in Gulf Stream and Deep Western Boundary Current (DWBC) transports, as compared to the LGM_Deep10yr and OCCA estimates (Figure 5-4). Unfortunately, the difference in transports between LGM_Deep10yr and LGM_Deep are much more related to forward model drift than they are a response of the optimization to the data constraints. To make this clearer, consider the evolution of the AMOC over the 80 years of the LGM_Deep estimate: the AMOC strength increases from about $8 \mathrm{~Sv}$ at year 5 to $22 \mathrm{~Sv}$ at year 80 . AMOC strength increases over the course of the estimate because Gulf Stream transport increases much more rapidly than does the surface return transport.

The AMOC is relatively shallow in all years of the LGM_Deep10yr and LGM_Deep estimates, and is thus a more consistent feature than is the net AMOC transport. There are several potential explanations for the relatively shallow depth of the AMOC. Most intriguing is the possibility that such a circulation is required by the MC08 data and is thus produced by control adjustments. Unfortunately, there are several other possibilities that can not be excluded at this time. The state estimation approach already showed a tendency for a shallow AMOC with increasing run length in modern state estimates (see Figure 322). Also, the first guess initial conditions impose a relatively strong increase in salinity 
with depth below $1000 \mathrm{~m}$; other first guess conditions should be tested in future work to characterize the sensitivity of the estimated circulation to the choice of initial conditions.

The preceding discussion has shown that the LGM_Deep estimate does not meet the requirements for an equilibrium ocean estimate: significant drifts in both NSST and the MOC over the course of the estimate indicate that the estimate is far from a steady state. Nonetheless, this estimate represents an important step for paleo ocean climate estimation. In the interest of informing future efforts in this area, the fit of the model to the MC08 $\delta^{13} \mathrm{C}_{\text {calcite }}$ and $\delta^{18} \mathrm{O}_{\text {calcite }}$ data is now examined.

\subsubsection{Modeled versus observed $\delta^{13} \mathrm{C}$}

Since the MC08 records were collected at a variety of longitudes, latitudes, and depths, visual analysis is not straightforward; the scatter plots shown in Figure 5-5 provide one approach to visualizing the $\delta^{13} \mathrm{C}_{\text {calcite }}$ model-data fit in the LGM_Deep10yr and LGM_Deep estimates. The LGM_Deep10yr estimate of $\delta^{13} \mathrm{C}_{\text {calcite }}$ has an acceptable fit to the data without obvious model biases. Moving from the shorter LGM_Deep10yr to the longer LGM_Deep, a general increase in scatter is seen, consistent with the increase in $\delta^{13} \mathrm{C}_{\text {calcite }}$ costs in Figure 5-2. A notable trend is evident in the middle plot - LGM_Deep is biased high for low observed values, and low for high observed values (i.e. the model does not reproduce the extreme low or high values seen in the data). The pattern is also apparent (albeit more weakly) in the misfit against depth (lower right-hand plot).

To compare $\delta^{13} \mathrm{C}$ in the model and observations, Equation 5.1 is used to estimate modeled $\delta^{13} \mathrm{C}_{\text {calcite }}$ at each grid point. As shown for 12 selected depth levels in Figure 5-6, the inferred LGM_Deep $\delta^{13} \mathrm{C}_{\text {calcite }}$ distributions have reasonable spatial structure (i.e. they are smooth rather than patchy), have the expected distribution with depth (i.e. higher values at the surface and lower values at depth), and the model estimates agree well in a qualitative sense with the MC08 records. Combining this mapped comparison with the AMOC discussion (Figure 5-4 and related discussions above), we surmise that the data are consistent 

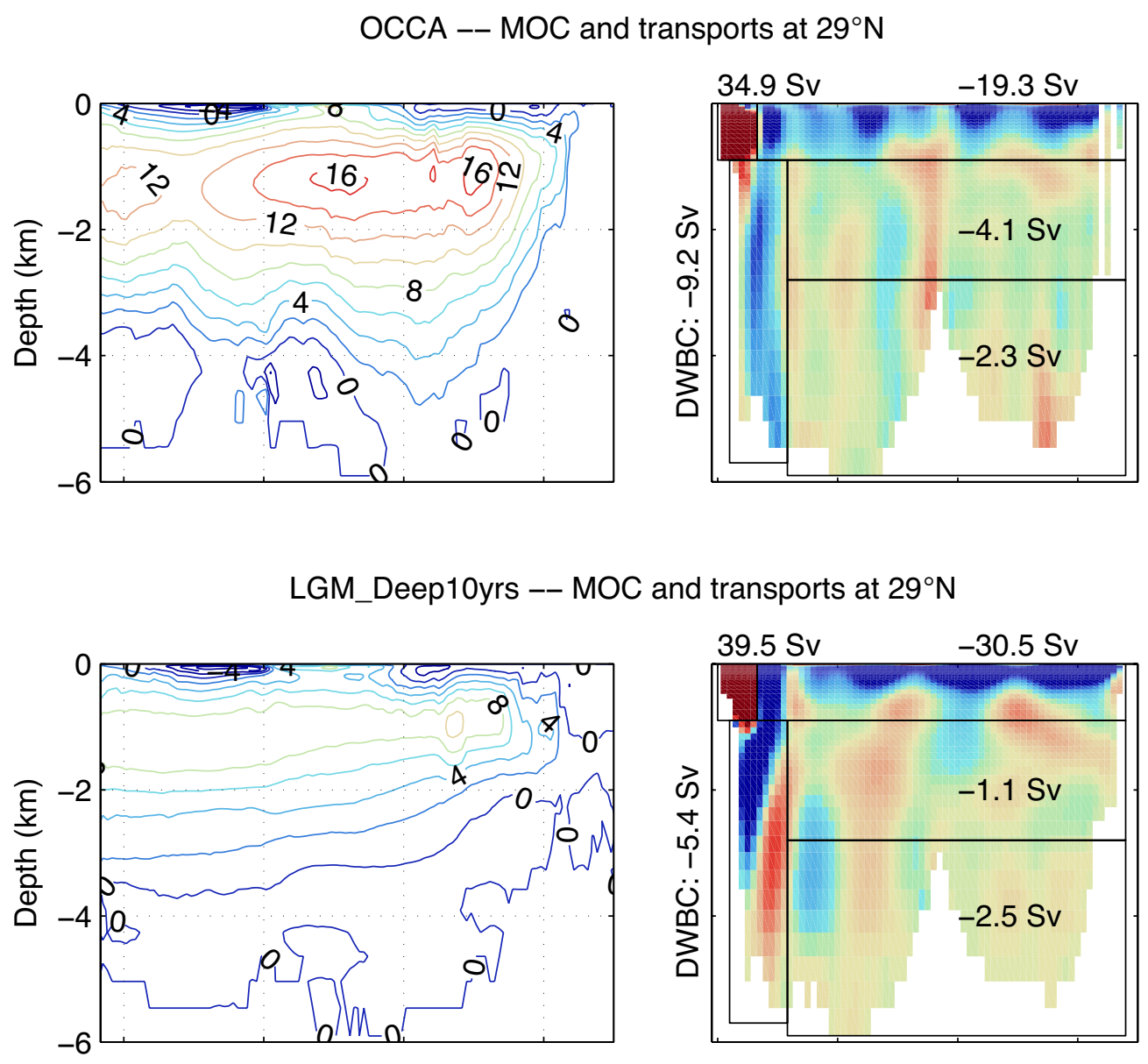

LGM_Deep -- MOC and transports at $29^{\circ} \mathrm{N}$
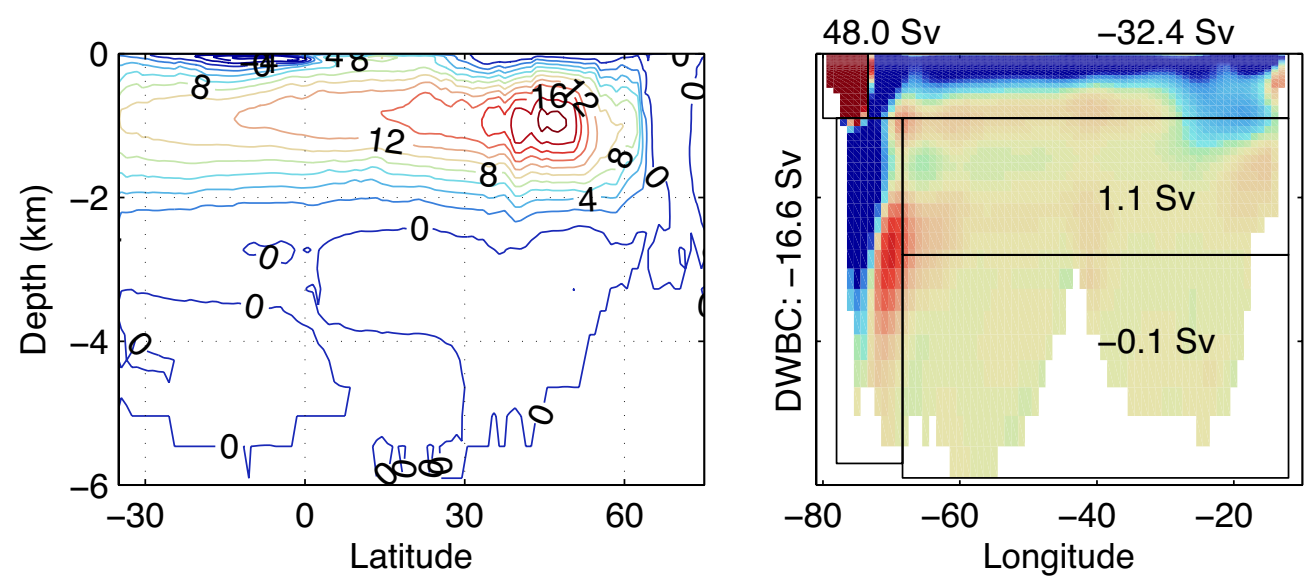

Figure 5-4: Zonally integrated overturning strength in Sverdrups (left) and meridional velocity at $29^{\circ} \mathrm{N}$ (right). Rows show OCCA (top), LGM_Deep10yr (middle) and LGM_Deep (bottom). Numbers at right give total transports in boxed regions. 
LGM_Deep10yrs
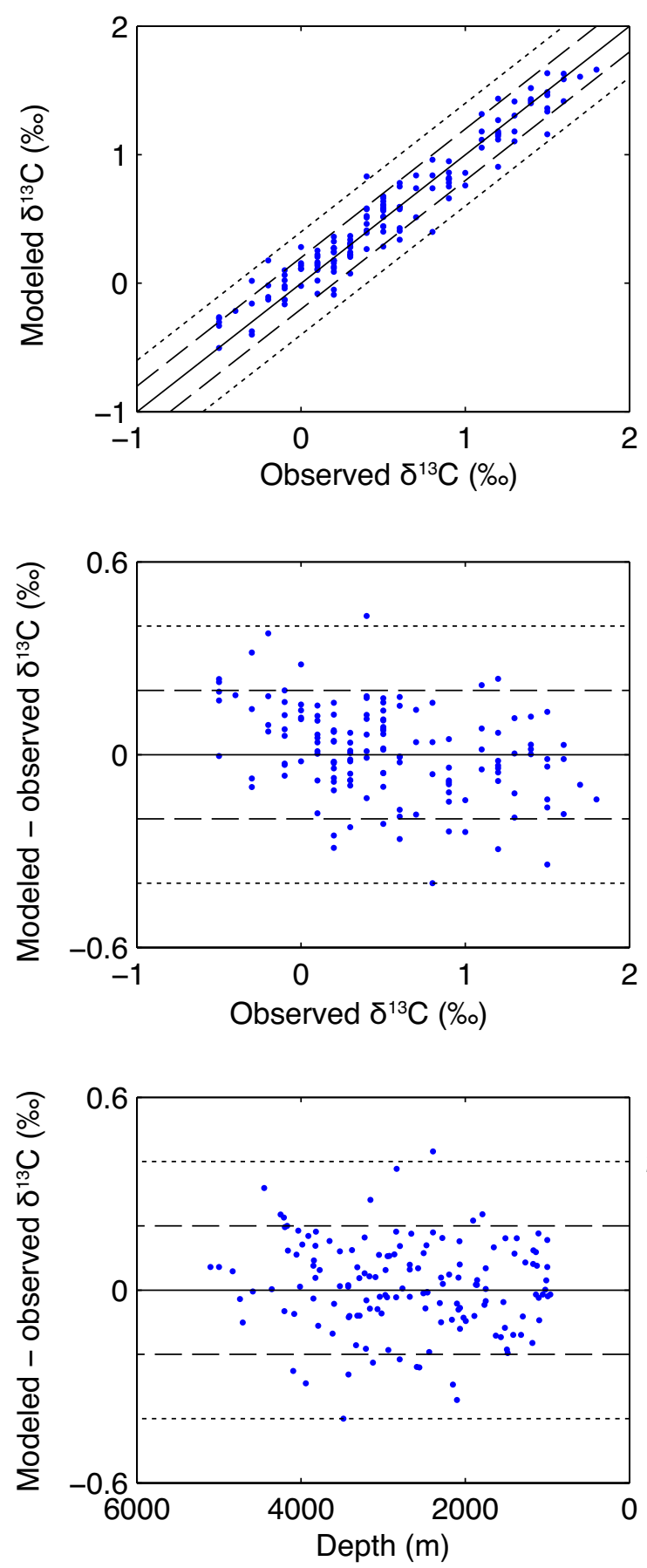

LGM_Deep
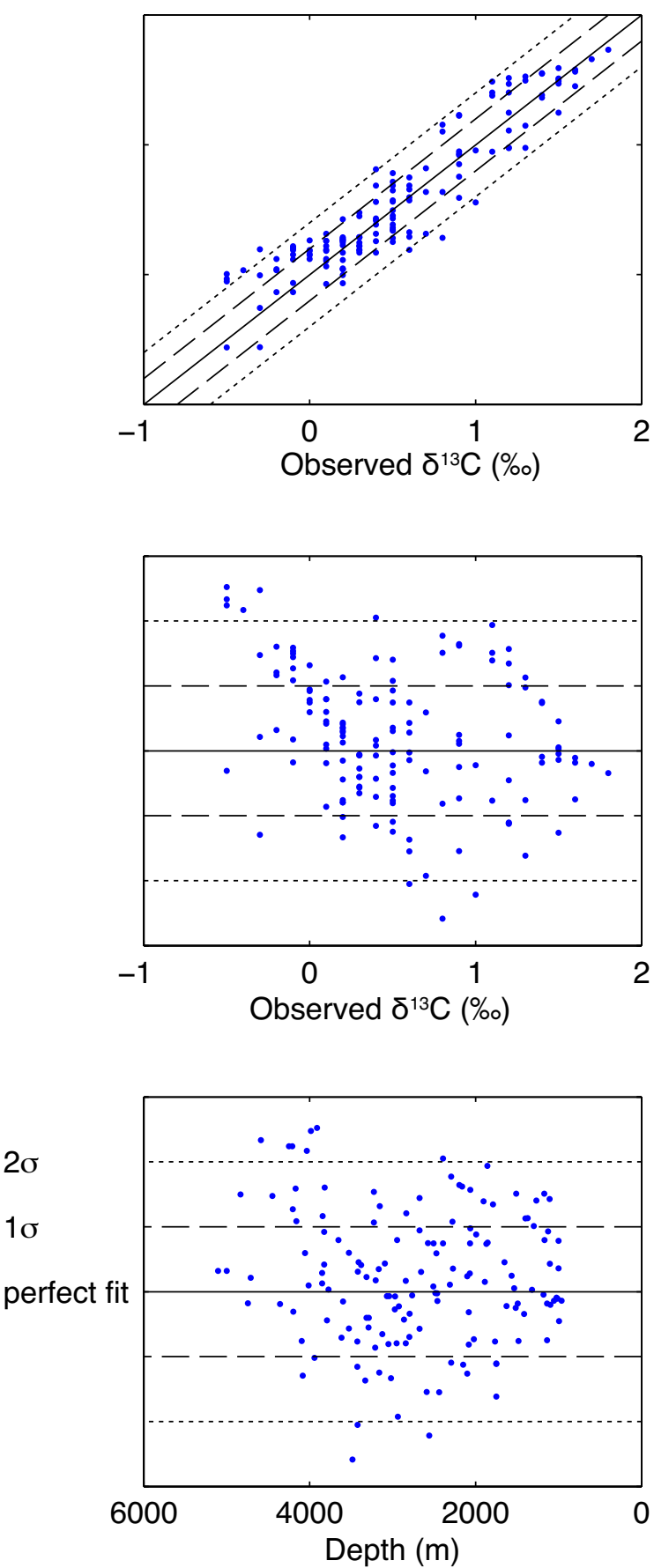

Figure 5-5: $\quad \delta^{13} \mathrm{C}_{\text {calcite }}$ model-data fit for LGM_Deep10yr (left) and LGM_Deep (right). Plots show modeled $\delta^{13} \mathrm{C}_{\text {calcite }}$ against the MC08 observations (top), misfit against observed values (middle), and misfit against observation depth (bottom, $\mathrm{x}$-axis reversed for comparison with middle row). Straight lines in all subplots show a perfect fit, $\pm 1 \sigma$, and $2 \sigma$. 
with a vigorous northern source branch of the AMOC above about $2000 \mathrm{~m}$, and with an ocean below $2500 \mathrm{~m}$ that has a nearly zero net meridional transport. It should be noted that the consistency of model and data is likely sensitive to the remineralization rate assumed in the model, and to the number of years over which the remineralization can influence modeled $\delta^{13} \mathrm{C}$. Thus, in a model with a different remineralization rate, or in one that is run out to equilibrium, the data could require a more vigorous lower branch than seen here. These possibilities should be explored in future work.

\subsubsection{Modeled versus observed $\delta^{18} \mathrm{O}$}

The fit of the model to the MC08 $\delta^{18} \mathrm{O}_{\text {calcite }}$ data is now examined. $\delta^{18} \mathrm{O}_{\text {calcite }}$ in the model is computed from modeled $\delta^{18} \mathrm{O}_{\text {water }}$ and temperature using Equation 5.2; the dual dependence of $\delta^{18} \mathrm{O}_{\text {calcite }}$ on $\left(\mathrm{T}, \delta^{18} \mathrm{O}_{\text {water }}\right)$ complicates interpretation and should be kept in mind in the following discussion. As shown in Figure 5-7 (left), LGM_Deep10yr modeled $\delta^{18} \mathrm{O}_{\text {calcite }}$ fits the MC08 records well with most records falling within $1 \sigma$ and nearly all records falling within $2 \sigma$. A bias is apparent in the middle plot - modeled $\delta^{18} \mathrm{O}_{\text {calcite }}$ is too high at the low end of the range and too low at the high end of the range; as for $\delta^{13} \mathrm{C}_{\text {calcite }}$, the model has difficulty capturing the full range seen in the data. In moving from LGM_Deep10yr to the longer LGM_Deep, misfits increase in all three scatter plots. The trend in the middle plot worsens, and the plot of misfits against depth (bottom plot) shows that misfits become particularly large at depths below $2000 \mathrm{~m}$.

Figure 5-8 compares the geographic distribution of modeled $\delta^{18} \mathrm{O}_{\text {calcite }}$ in LGM_Deep against the MC08 proxy records for 12 selected depth levels. LGM_Deep $\delta^{18} \mathrm{O}_{\text {calcite }}$ values are low in the upper ocean and high in the deep ocean, in keeping with the proxy records. At two depth levels, 1357-1461 m and 1696-1835 m, the LGM_Deep estimate provides a particularly poor fit to the data. As these depths are strongly influenced by waters originating in the north in this estimate, these misfits may be evidence that the state estimation approach has difficulty controlling the temperatures of water masses formed in the north. A similar observation was made in the context of building longer modern state estimates 

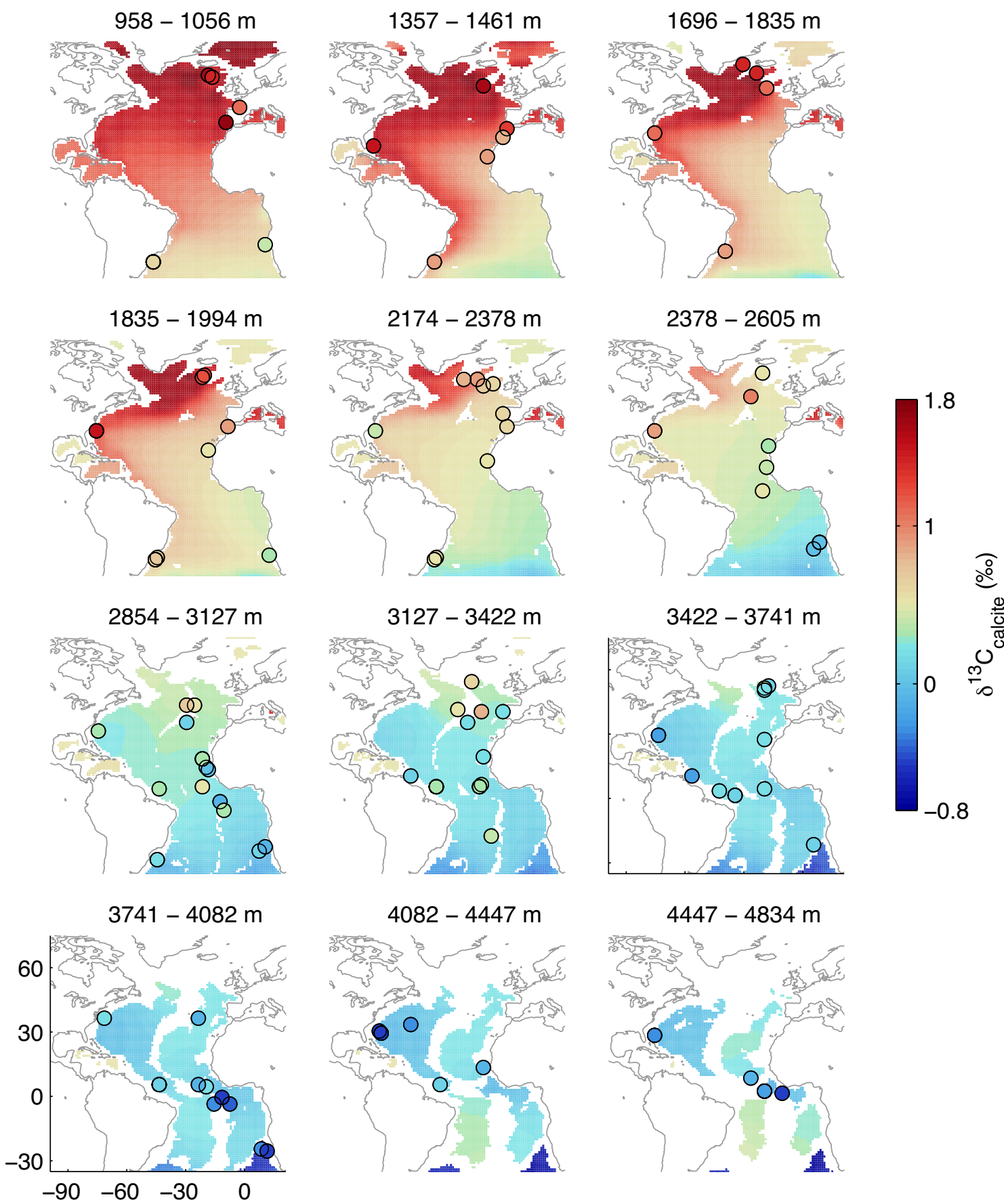

Figure 5-6: Inferred $\delta^{13} \mathrm{C}_{\text {calcite }}$ fields in LGM_Deep at selected model depths; MC08 records that fall in each depth range are overlaid. There are 21 model levels for which $\delta^{13} \mathrm{C}$ is constrained; the 12 shown were chosen to provide coverage of the full depth range and because there were significant numbers of proxy records mapped to these levels. 
LGM_Deep10yrs
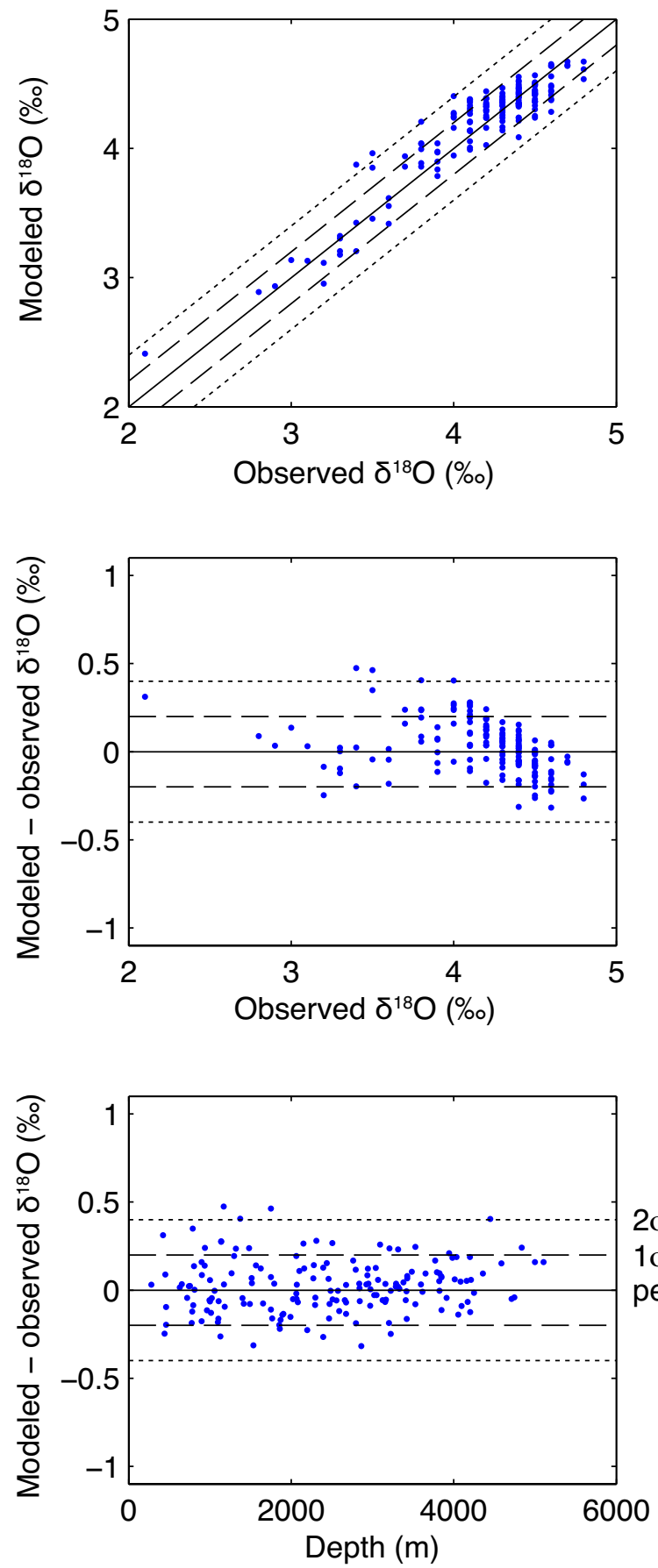

LGM_Deep
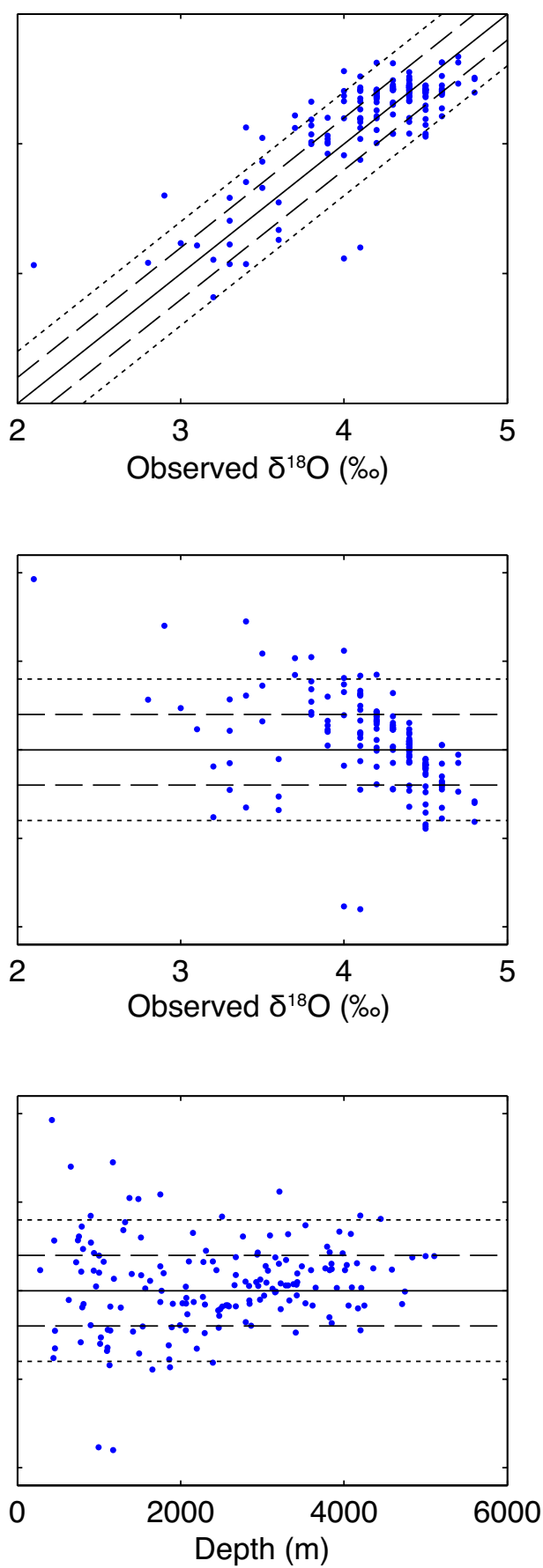

Figure 5-7: Model-data fit for $\delta^{18} \mathrm{O}_{\text {calcite }}$ for LGM_Deep10yr (left) and LGM_Deep (right). Plots show modeled $\delta^{18} \mathrm{O}_{\text {calcite }}$ against the MC08 observations (top), misfit against observed values (middle), and misfit against observation depth (bottom). Straight lines in all plots show a perfect fit, $\pm 1 \sigma$, and $2 \sigma$. 
(see Figure 3-24). Below about 2800 m, model-data misfits appear larger than they are due to the tight range of $\delta^{18} \mathrm{O}$ included in the colorscale. Indeed, the lower right-hand scatter plot of Figure 5-7 shows that nearly all of the data below $2800 \mathrm{~m}$ are fit within $\pm 2 \sigma$ by the LGM_Deep estimate.

\subsection{Comparison to previous studies}

Although there are many forward models of general ocean circulation at the LGM (such as the PMIP models), there are many fewer model estimates of LGM $\delta^{13} \mathrm{C}$ and $\delta^{18} \mathrm{O}$ distributions (see Section 2.3.2 for a review); to our knowledge, model output is not publicly available for the model results that do exist. Most previous forward models of LGM $\delta^{13} \mathrm{C}$ and $\delta^{18} \mathrm{O}$ have been compared only qualitatively to data, making it difficult to compare the skill of these models to the skill of the approach used here. Nonetheless, there is at least one recent model with which a more direct comparison is possible. Section 2.3 described the Hesse et al. [2011] forward model of $\delta^{13} \mathrm{C}$ and the application of the model to estimate LGM $\delta^{13} \mathrm{C}$ distributions; scatter plots of the Hesse et al. [2011] modeled LGM $\delta^{13} \mathrm{C}$ against LGM $\delta^{13} \mathrm{C}$ proxies were shown in Figure 2-10.

The fit of LGM_Deep to the data appears to be better than that of the three Hesse et al. [2011] scenarios; to see this, compare Figure 2-10 with Figure 5-5 (note that the $2 \sigma$ uncertainty ranges are less strict in Figure 2-10 than in Figure 5-5: $\pm 0.5 \%$ vs $\pm 0.4 \% 0$ ). There are many differences between Hesse et al. [2011] model and the LGM_Deep estimate: complexity of the physical model (Hesse et al. use a much less complete intermediate complexity model), complexity of the biogeochemistry and isotope models (LGM_Deep uses a much simpler model), level of equilibration (the Hesse et al. 2011 simulations are much closer to equilibrium), and whether the model is data-constrained (the Hesse et al. 2011 simulations are not data-constrained). Although beyond the scope here, much could be learned about how to improve these models by comparing results obtained in such different ways. 


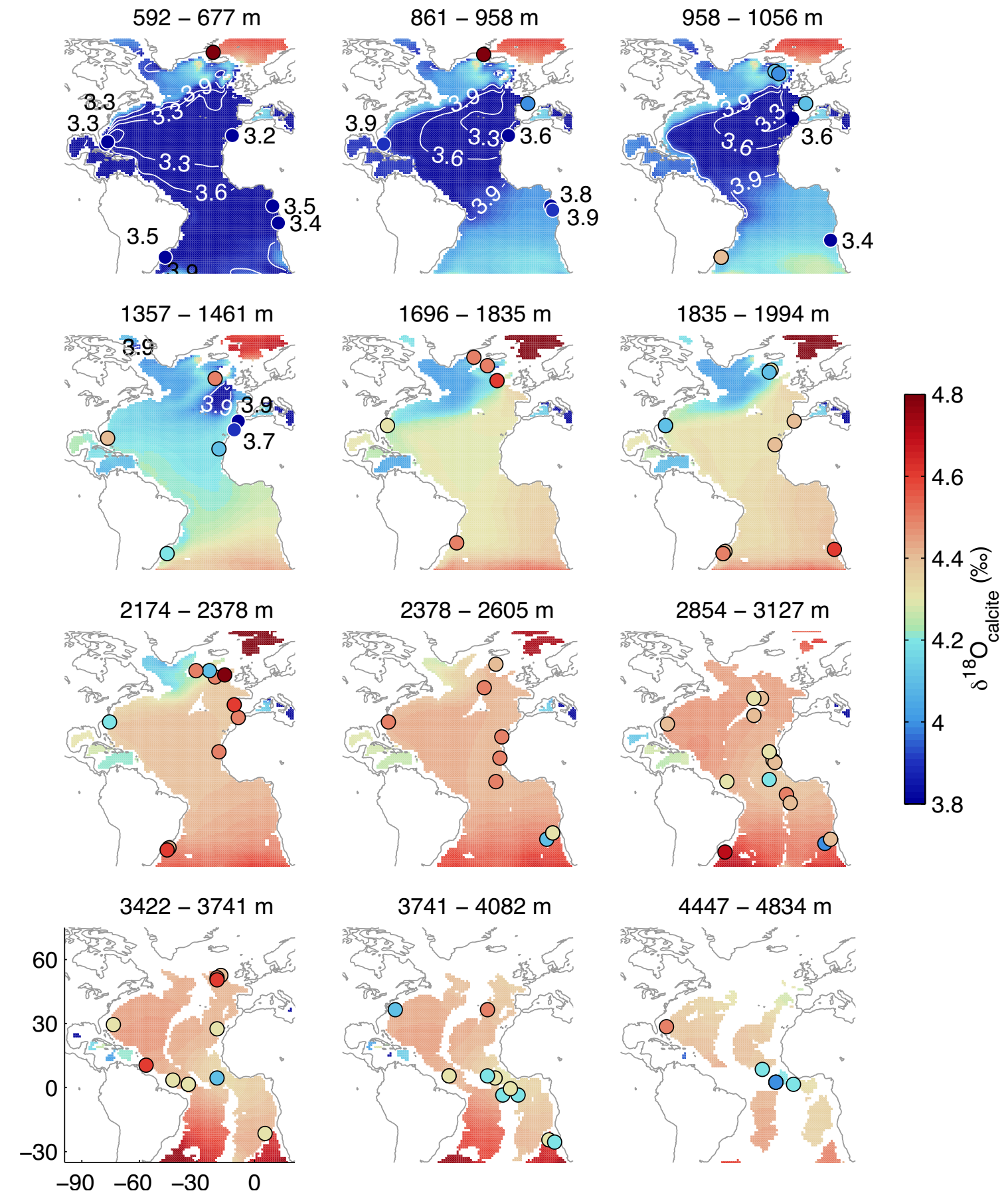

Figure 5-8: Inferred $\delta^{18} \mathrm{O}_{\text {calcite }}$ fields in LGM_Deep at selected model depths; MC08 records that fall in each depth range are overlaid. For tracer levels below the blue end of the colormap, white contour lines are drawn at $\delta^{18} \mathrm{O}_{\text {calcite }}=(3,3.3,3.6$ and 3.9) and MC08 values are written in black next to the data location. 


\subsection{Chapter summary}

This chapter has focused on reconstructing deep ocean conditions at the LGM. An 80-year LGM state estimate, LGM_Deep, has been developed that is constrained by MARGO data in addition to benthic $\delta^{13} \mathrm{C}_{\text {calcite }}$ and $\delta^{18} \mathrm{O}_{\text {calcite }}$ records. Due to technical limitations, this estimate does not satisfy the requirements we have identified for ocean climate estimates; the estimate has substantial drift in NSSTs, and the AMOC transport increases significantly over the course of the 80 year estimate (due to an increased surface return flow). Although LGM_Deep must be considered preliminary, results are encouraging. The fit of the estimate to the MARGO and benthic $\delta^{13} \mathrm{C}_{\text {calcite }}$ and $\delta^{18} \mathrm{O}_{\text {calcite }}$ data is satisfactory or close to satisfactory for all proxy types. The spatial distributions of modeled $\delta^{13} \mathrm{C}_{\text {calcite }}$ and $\delta^{18} \mathrm{O}_{\text {calcite }}$ appear realistic (i.e. smooth and large-scale, not patchy) and generally show good visual agreement with the data. As compared to a forward $\delta^{13} \mathrm{C}$ calculation using an intermediate complexity forward model [Hesse et al., 2011], the LGM_Deep estimate appears to provide a better fit to proxy records.

Considering the limitations of the estimate, LGM_Deep is presented as an exploratory estimate - it demonstrates many of the strengths and challenges of applying ocean state estimation to paleoclimate, and it provides a stepping stone for future estimates of paleo ocean states. 


\section{Chapter 6}

\section{Discussion}

This thesis has touched on diverse topics including modern ocean state estimation, forward models and proxy records of the Last Glacial Maximum, and estimation of ocean state at the LGM by combining models and data. This chapter begins with a long-term view of the future of ocean state estimation in Section 6.1. Novel contributions of the thesis are summarized in Section 6.2, and Section 6.3 describes limitations of the thesis and promising avenues for future research. A closing for the thesis is given in Section 6.4.

\subsection{A vision for ocean state estimation and equilibrium ocean estimation}

Estimation of the climate state using data and models together will only become more important with time. Regardless of improvements in available data, observations will continue to have uncertainties and biases, and there will always be climate-relevant properties that cannot be directly observed (e.g., integrated net heat flux in the ocean and atmosphere, and integrated ocean uptake of anthropogenic carbon). Regardless of their sophistication, climate models will continue to have poorly known parameters and missing physics. The long-term future of state estimation likely lies in fully coupled ocean / atmosphere / cryosphere / biosphere models that are constrained by a wide diversity of datasets. Avail- 
ability of such tools may be many years away, and the machinery of state estimation may be very different by then. Nonetheless, the general principle is likely to apply - the best estimates of climate can come through a combination of data and known physical principles.

There are a number of scientific problems that cannot be solved by estimating the transient ocean state over a specific time period with instrumental observations, as is usually done in modern ocean state estimation. There are (at least) two types of problems for which properties of interest cannot be meaningfully constrained by available data: (1) problems for which the timescales of interest are too long as compared to the observational record, and (2) problems for which data are too sparse. An example of the first type of problem is the calculation of the time required to reach tracer equilibrium [Wunsch and Heimbach, 2008]. Problem type 2 has been a dominant theme of this thesis - LGM proxy records are geographically sparse, and do not provide the temporal resolution to adequately constrain any temporal variability (other than mean seasonal properties). For these types of problems, it is typically assumed at the outset that the circulation of interest is in some way steady in time; assuming a steady circulation drastically reduces the degrees of freedom in the physical system, making progress possible in severely data-limited scenarios. Possible assumptions vary from fully steady with no seasonal cycle (e.g., Marchal and Curry 2008), a repeating 17 year cycle of modern ocean state (e.g., Wunsch and Heimbach 2008), or permitting all temporal variability captured by coupled climate models and running them out to quasi-equilibrium (e.g., Braconnot et al. 2007a).

In the long term, techniques for equilibrium ocean estimation (EOE), or more generally, equilibrium climate estimation (ECE), are required. What is needed are climate estimates that are consistent with (1) known dynamics (given uncertainties in the dynamical representation), (2) available data (within their uncertainties) and (3) a steady state assumption (whether fully steady state or cyclic in nature). In this thesis, the approach taken by LGM climate modelers was adopted - that a long-running model simulation with limited drift is indicative of a quasi-steady circulation in which atmospheric forcing and ocean conditions are in quasi-equilibrium. Thus, we sought long running ocean state estimates with limited 
drift. There are many likely other avenues that could be pursued to achieving the three goals of EOE simultaneously in one state estimate, and it is likely that other, potentially very different, approaches will be developed in the coming years.

\subsection{Novel contributions of the thesis}

Upper ocean conditions at the LGM. A key contribution of this thesis is the development of an upper ocean state estimate (LGM_Upper) that is consistent with ocean dynamics and with the MARGO near sea surface temperature (NSST) data. Uncertainties published by the MARGO project were reported with an unknown multiplicative factor, which was assigned a value of one as a reasonable first estimate. In this thesis, the MARGO data are shown to be inconsistent with each other given this estimated uncertainty. Consistency between the model and all MARGO datasets is achieved for the LGM_Upper state estimate by assigning a factor of two to alkenone and dinocyst uncertainties north of $40^{\circ} \mathrm{N}$ and a factor of one to all other MARGO data. The LGM_Upper estimate has a much better fit to the MARGO data than do the PMIP2 coupled models of LGM climate. This thesis shows that the PMIP2 models are not consistent with the MARGO data and their published errors, in contrast to an analysis by Hargreaves et al. [2011] that relied on a heavily smoothed and averaged MARGO NSST product. In a comparison against sea ice proxy records (data that were withheld from the state estimate), this thesis shows that the sea ice distribution of one of the PMIP2 models is clearly inconsistent with the proxy records. Given large uncertainties in the data, as well as disagreement between foram-based and dinocyst-based sea ice reconstructions, available data appear insufficient to accept or reject the sea ice distribution in the other PMIP2 models or in the LGM_Upper estimate. Finally, this thesis showed that moderate changes to modern atmospheric conditions are sufficient to fit LGM NSST records.

Equilibrium ocean estimation. This thesis develops equilibrium ocean estimation (EOE) as a concept, and demonstrates the need for EOE through the problem of recon- 
structing the deep ocean circulation at the LGM. Long state estimation periods, facilitated by asynchronous timestepping and carry-over control, are proposed as a method of achieving EOE goals. In both modern and LGM scenarios, longer state estimation periods are achieved than in previously published ocean state estimates.

State estimation with $\delta^{13} \mathbf{C}$ and $\delta^{18} \mathrm{O}$ data in the MIT GCM. Prior to this thesis, $\delta^{13} \mathrm{C}$ and $\delta^{18} \mathrm{O}$ had not been used in MIT GCM ocean state estimates, nor even in forward models using the MIT GCM. A simple approach to treatment of $\delta^{13} \mathrm{C}$ and $\delta^{18} \mathrm{O}$, based heavily on that of Marchal and Curry [2008], has been added to the MIT GCM and the state estimation machinery has been augmented with appropriate controls for $\delta^{13} \mathrm{C}$ and $\delta^{18} \mathrm{O}$ initial and boundary conditions. In Chapter 3, these approaches are applied to reconstruct full 3-dimensional fields of (1) modern $\delta^{13} \mathrm{C}_{\mathrm{DIC}}$ based on sparse GEOSECS $\delta^{13} \mathrm{C}_{\mathrm{DIC}}$ measurements and (2) modern $\delta^{18} \mathrm{O}_{\text {water }}$ based on sparse $\delta^{18} \mathrm{O}_{\text {water }}$ data from Schmidt et al. [1999]. In Chapter 5 , benthic $\delta^{13} \mathrm{C}_{\text {calcite }}$ and $\delta^{18} \mathrm{O}_{\text {calcite }}$ records compiled by Marchal and Curry [2008] are used to estimate deep ocean circulation at the LGM. Inferred 3-dimensional fields of LGM $\delta^{13} \mathrm{C}_{\text {DIC }}$ and $\delta^{18} \mathrm{O}_{\text {water }}$ are shown to be qualitatively reasonable (i.e. largescale and smooth), and to be consistent or close to consistent with the proxy records. These results indicate that the Marchal and Curry [2008] LGM compilation may be consistent with a shallow but strengthened AMOC at the LGM, although other arrangements cannot be excluded. Finally, it appears that the LGM_Deep estimate provides a closer fit to LGM $\delta^{13} \mathrm{C}_{\text {calcite }}$ data than does the Hesse et al. [2011] model estimates of LGM $\delta^{13} \mathrm{C}$.

\subsection{Limitations and opportunities for future progress}

A number of serious limitations in current approaches to studying the LGM Atlantic Ocean have been highlighted in this thesis. These are briefly reviewed for LGM data and models in general, and then more specific limitations are discussed for the results of the thesis.

Data. Key issues with currently available proxy data of the LGM Atlantic Ocean include data sparsity, poorly known uncertainties in the data, disagreements between different 
proxy types, and a lack of reliable proxies for key ocean characteristics (such as salinity). Canonical values for uncertainty on LGM NSST proxy records are $1-2^{\circ} \mathrm{C}$; if all of the MARGO NSST records are to be treated equally, disagreements between datasets clearly show these estimates are too conservative. Better constraints on the interpretive error of the proxies are critical - e.g. how well-known is the calcification depth, the season recorded by the proxy, and whether the proxy records average or peak conditions. Studies that seriously consider diverse proxy types together, such as the MARGO project, are important.

Models. Key issues with numerical models include model resolution, parameterizations of subgrid-scale physics with poorly known parameters, missing physics, errors in the numerical solution of the differential equations of motion, and poorly known initial and boundary conditions. On the modeling side, the question of whether modern climate models can adequately represent diverse climates, such as that of the LGM, is a pivotal one. These same models are being used to predict future climate change, but their adequacy outside of the modern climate is poorly assessed. Recently, paleoclimate scenarios have been included in IPCC simulations for the first time [Braconnot, 2011], and serious efforts are underway to evaluate these models against proxy records [Braconnot et al., 2012]. These are clear steps in the right direction.

LGM model-data synthesis. Much work remains in the area of model-data synthesis for studying paleoclimates. Data assimilation is a relatively young field, and the practical application of the adjoint method to estimate modern ocean state has been possible for only 10-15 years. Indeed, only one study prior to this thesis has applied the adjoint method to estimate past climates with a general circulation model and proxy records. Methods will likely improve as additional experience is gained.

The state estimation approach used in this thesis has a number of key limitations. It has not been possible to build full equilibrium ocean estimates - the techniques used here do not appear to permit identification of a model state that is consistent with (1) a steady state assumption, (2) available data, and (3) known ocean dynamics. Specific issues include the following: it has not been possible to reach estimation periods longer than 80 years 
(longer simulations are possible, but are essentially unconstrained by data); 80 year LGM state estimates show significant drift in temperatures, salinities, and AMOC strength; and AMOC strength is persistently weak and shallow in modern state estimates 10 years in length or longer.

Estimates such as those produced by the ECCO project show that it is possible to obtain time-varying estimates of ocean state that are consistent with the observational record and with known ocean dynamics, and previous LGM inverse modeling studies show that it is possible to obtain estimates that are consistent with the data, limited dynamics, and a built-in assumption of steady state. What is missing is approaches that satisfy all three of the equilibrium ocean estimation requirements at once. There are many potential avenues to future progress. The most obvious is to compare the results achieved here with those achieved using other model-data synthesis techniques. Recently, G. Gebbie has used similar LGM datasets to constrain water mass distributions and pathways in a kinematic model (see page 83 for a discussion). That approach uses a structural steady state assumption and limited dynamics, but is able to identify a steady state solution that is consistent with many LGM proxy records. Each approach satisfies 2 of the 3 desired requirements of an OCE - by comparing them perhaps an approach can be identified that satisfies all 3 in a single estimate.

There are also promising avenues for more directly improving the LGM state estimates presented in this thesis. An important unknown is how sensitive the solutions are to key assumptions such as the choice of initial and boundary conditions, and the assignment of uncertainties to these conditions. Additional LGM state estimates should be computed starting from different prior assumptions. One area of particular interest is the importance of the first guess atmospheric forcing. The thesis employs a single year of NCEP as a first guess, but other types of atmospheric forcing should be tested. A first step has been made in this direction: we have successfully used CCSM3 PMIP2 LGM atmospheric forcing and initial conditions in forward runs using the MIT GCM. Unfortunately, the optimization is not stable in this setup. It is possible that this is simply a technical issue that can be resolved 
with additional effort; if not, it will be important to understand why the optimization can not be effectively applied to this model configuration. Another key test will be to study the sensitivity of the final estimate to the first guess initial conditions. We have used a first guess salinity field that has particularly high salinity in deeper layers, but the extra salt was added in distinct layers. Other choices should be tested, such as a haline stratification like today's, or a salinity field in which deeper layers are made saltier using a continuous, rather than step-wise, approach. Another key unknown is the extent to which an ocean circulation can be constrained given the sparse and uncertain nature of proxy observations. Pseudo-proxy experiments will be important to determining this; one could use one of the LGM state estimates presented in this thesis as first guess initial and boundary conditions, constrain a new modern estimate with modern data sub-sampled at LGM proxy locations (with added noise), and evaluate how close the final state estimate is to modern conditions.

\subsection{Closing}

The study of past ocean circulation has historically been largely driven by two main approaches: (1) proxy record development with a sometimes qualitative consideration of ocean dynamics, and (2) modeling of ocean circulation with a sometimes biased selection of preferred data and model-data comparisons that are often qualitative in nature. Quantitative synthesis of ocean dynamics and data is a challenge: building a consistent picture requires full consideration of the complexities and uncertainties of both the observational record and numerical models of ocean circulation. Facing these challenges is necessary if the full promise of paleoclimate is to be realized: that past climates can provide quantitative evidence to aid in the difficult but necessary task of understanding how climate may respond to anthropogenic forcings in the future. 


\section{Appendix A}

\section{MARGO uncertainty assignments}

The Multi Proxy Approach for the Reconstruction of the Glacial Ocean (MARGO) project developed uncertainty assignments for each near sea surface temperature (NSST) proxy record included in the compilations produced by the project [Waelbroeck et al., 2009]. Given the important role of uncertainty estimates in least squares minimization, the MARGO approach to uncertainty estimation is reviewed.

MARGO uncertainties are given by

$$
\sigma_{M A R G O}=f a c \cdot \sigma_{c a l} \cdot q
$$

where $\sigma_{c a l}$ is a mean calibration error and $q$ is a mean reliability index; these will be described in the following paragraphs. $f a c$ is a multiplicative factor which is assigned a value of one as a reasonable first estimate [Waelbroeck et al., 2009]; the inclusion of this unknown factor by Waelbroeck et al. [2009] is an indication of how difficult quantitative uncertainty estimation is for proxy records. MARGO-defined uncertainties are also termed semi-quantitative, because they depend on both qualitative and quantitative metrics for data uncertainty, as will be clear in the following paragraphs. Figure A-1 shows the distribution and mean of $\sigma_{M A R G O}$ for each proxy type and each season. 

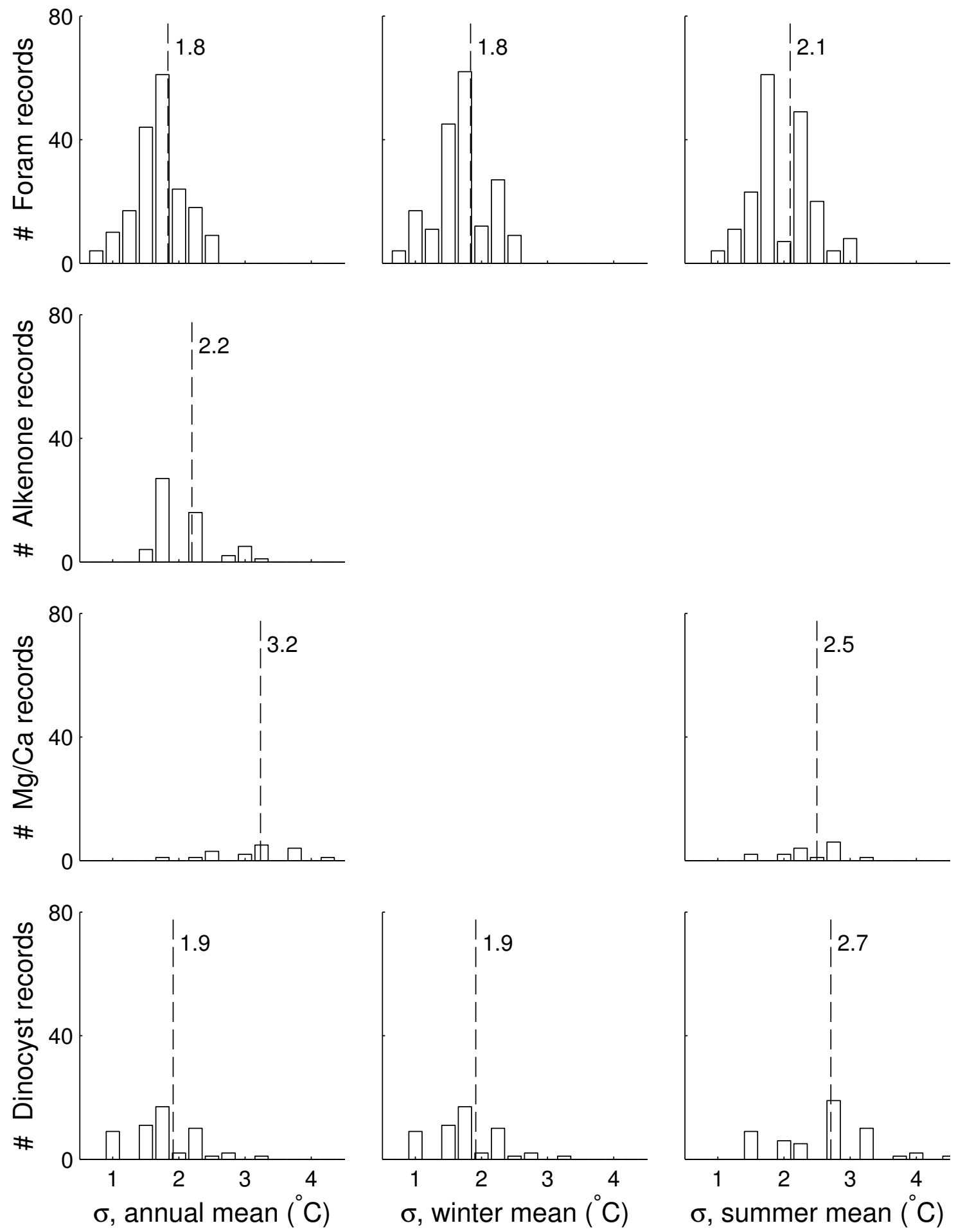

Figure A-1: Histograms of semi-quantitative uncertainties defined by the MARGO project for each proxy and season. All axes have the same limits. Labeled dashed lines give the mean uncertainty for each histogram. 
As described by Waelbroeck et al. [2009], the calibration error $\left(\sigma_{c a l}\right)$ was defined in one of two ways in MARGO, depending on the dataset. For proxy reconstructions that rely on a calibration equation, the standard error of the residuals of the calibration equation is used. For reconstructions that rely on assemblage-based approaches, one or more modern records are temporarily excluded from the reference database; their properties are predicted based on the other records; and the actual and predicted values are used to compute the root-meansquared error of prediction. Foraminifera calibration errors are specific to each record and vary from 0.8 to $1.5^{\circ} \mathrm{C}$; alkenone and $\mathrm{Mg} / \mathrm{Ca}$ calibration errors are taken to be $1.5^{\circ} \mathrm{C}$ for all records; and dinocyst calibration errors are $1.2^{\circ} \mathrm{C}$ for annual mean, $1.2^{\circ} \mathrm{C}$ for winter mean, and $1.7^{\circ} \mathrm{C}$ for summer mean conditions.

The mean reliability index was defined as

$$
q=\left(0.75 * q_{\text {str }}+q_{\text {num }}+q_{\text {rel }}\right) / 2.75
$$

where quality flags are based on chronostratigraphic quality $\left(q_{s t r}\right)$, number of samples used to compute the reconstructed value $\left(q_{\text {num }}\right)$, and a reliability index $\left(q_{\text {rel }}\right) . q_{\text {num }}$ and $q_{\text {rel }}$ are assigned values from 1 to 3 , while $q_{s t r}$ takes values from 1 to 4 ; $q$ is computed as a weighted mean of these components where each is normalized by its range. Chronostratigraphic quality $q_{s t r}$ is assigned values of 1-4 [De Vernal et al., 2005]. Level 1 indicates chronologic control is provided through either annually counted layers or two radiometric dates in the 19-23 cal kyr period. Level 2 indicates control is provided by two radiometric dates within 12-30 ka, or that correlation of properties to another core dated with protocol 1 is used. Level 3 indicates control is based on other stratigraphic constraints that are correlated to records dated at quality level 2. $q_{\text {num }}$ is assigned a value of 1, 2, or 3 when the number of samples in the LGM interval is $>4,2-4$, or exactly 1 , respectively. The reliability level $q_{r e l}$ is assigned values between one and three; the assignment is proxyspecific and was chosen as follows. For dinoflagellate cysts, $q_{r e l}=1,2$, and 3 indicate analogue situations that are good, acceptable and poor, respectively [Waelbroeck et al., 2009]. For foraminiferal assemblage-based reconstructions, NSST estimates generated by 
4 reconstruction techniques are compared. $q_{r e l}=1,2$, and 3 indicate a maximum difference among the 4 techniques of $<2,2-4$ and $>4{ }^{\circ} \mathrm{C}$, respectively. For dinoflagellate cyst assemblage-based reconstructions, values of $q_{r e l}=1,2$, and 3 are assigned for "distances" between sample and best analog of $<37,37-74$, and $>74$ (distance is a metric specific to the dinocyst reconstruction approach, see De Vernal et al. [2005]). For Mg/Ca, assignments are basin-specific. For the Atlantic, $q_{\text {rel }}=1$ is assigned for core depths shallower than 3000 $\mathrm{m}$ and for near-surface dwelling species; $q_{\text {rel }}=2$ for core depths larger than $3000 \mathrm{~m}$ or non-near surface dwelling species; and $q_{\text {rel }}=3$ for cases where the core is deeper than $3000 \mathrm{~m}$ and the species is not surface dwelling. Uncertainties are larger for species that are not surface dwelling as any change in stratification between the LGM and today would make the reconstructed near sea surface temperatures less reliable. Uncertainty is increased with depth because post-depositional dissolution increases with depth and introduces a bias on temperatures reconstructed with $\mathrm{Mg} / \mathrm{Ca}$ (see Section 2.2.2). 


\section{Bibliography}

A. Adcroft, C. Hill, J. Campin, J. Marshall, and P. Heimbach. Overview of the formulation and numerics of the MIT GCM. Proc. of the ECMWF Seminar Series on Numerical Methods, Recent Developments in Numerical Methods for Atmosphere and Ocean Modelling, ECMWF, pages 139-149, 2004.

A. J. Adcroft, C. N. Hill, and J. C. Marshall. A new treatment of the Coriolis terms in C-Grid models at both high and low resolutions. Mon. Weather Rev., 127:1928-1936, 1998.

J. F. Adkins and D. P. Schrag. Pore fluid constraints on deep ocean temperature and salinity during the Last Glacial Maximum. Geophys. Res. Lett., 28:771-774, 2001.

J. F. Adkins and D. P. Schrag. Reconstructing Last Glacial Maximum bottom water salinities from deep-sea sediment pore fluid profiles. Earth Planet. Sci. Lett., 216:109-123, 2003.

J. F. Adkins, K. McIntyre, and D. Schrag. The salinity, temperature, and $\delta^{18} \mathrm{O}$ of the glacial deep ocean. Science, 298:1769-1773, 2002.

A. Arakawa and V. Lamb. Computational design of the basic dynamical processes of the UCLA general circulation model. Methods in Computational Physics, 17:174-267, 1977.

J. Aristegui, S. Agusti, J. J. Middelburg, and C. M. Duarte. Chapter 10: Respiration in the mesopelagic and bathypelagic zones of the oceans. In P. del Gorgio and P. Williams, editors, Respiration in Aquatic Ecosystems, pages 181-205. Oxford University Press, 2004.

T. Arsouze, A. M. Treguier, S. Peronne, J.-C. Dutay, F. Lacan, and C. Jeandel. Modeling the Nd isotopic composition in the North Atlantic basin using an eddy-permitting model. Ocean Science, 6(3):789-797, 2010.

M. Baringer and J. Larsen. Sixteen years of Florida Current transport at $27^{\circ} \mathrm{N}$. Geophys. Res. Lett., 28(16):179-183, 2001. 
S. Barker, I. Cacho, H. Benway, and K. Tachikawa. Planktonic foraminiferal $\mathrm{Mg} / \mathrm{Ca}$ as a proxy for past oceanic temperatures: a methodological overview and data compilation for the Last Glacial Maximum. Quaternary Sci. Rev., 24(7-9):821-834, 2005.

H. Beltrami and J.-C. Mareschal. Simultaneous inversion of borehole temperature data for past climate determination. Geophys. J. Int., 129:311-318, 1997.

B. Bemis, H. Spero, J. Bijma, and D. Lea. Reevaluation of the oxygen isotopic composition of planktonic foraminifera: Experimental results and revised paleotemperature equations. Paleoceanography, 13(2):150-160, 1998.

W. R. Boos. Thermodynamic scaling of the hydrological cycle of the Last Glacial Maximum. J. Climate, 25:992 - 1006, 2012.

N. Bouttes, D. Paillard, D. Roche, V. Brovkin, and L. Bopp. Last Glacial Maximum $\mathrm{CO}_{2}$ and $\delta^{13} \mathrm{C}$ successfully reconciled. Geophys. Res. Lett., 38:L02705, 2011.

E. Boyle. Cadmium: Chemical tracer of deepwater paleoceanography. Paleoceanography, 3(4):471-489, 1988.

P. Braconnot. Paleoclimate Modeling Intercomparison Project (PMIP): proceedings of the third PMIP workshop. In P. Braconnot, editor, In WCRP-111, WMO/TD-1007, page 271, 2000 .

P. Braconnot. The Paleoclimate Modeling Intercomparison Project contribution to CMIP5. CliVAR Exchanges, 16(2):15-19, 2011.

P. Braconnot, B. L. Otto-Bliesner, S. Harrison, S. Joussaume, J.-Y. Peterchmitt, A. AbeOuchi, M. Crucifix, E. Driesschaert, T. Fichefet, C. Hewitt, M. Kageyama, A. Kitoh, A. Laîné, M.-F. Loutre, O. Marti, U. Merkel, G. Ramstein, P. Valdes, S. L. Weber, Y. Yu, and Y. Zhao. Results of PMIP2 coupled simulations of the Mid-Holocene and Last Glacial Maximum; Part 1: experiments and large-scale features. Clim. Past, 3(2):261277, 2007a.

P. Braconnot, B. L. Otto-Bliesner, S. Harrison, S. Joussaume, J.-Y. Peterchmitt, A. AbeOuchi, M. Crucifix, E. Driesschaert, T. Fichefet, C. Hewitt, M. Kageyama, A. Kitoh, M.-F. Loutre, O. Marti, U. Merkel, G. Ramstein, P. Valdes, S. L. Weber, Y. Yu, and Y. Zhao. Results of PMIP2 coupled simulations of the Mid-Holocene and Last Glacial Maximum; Part 2: feedbacks with emphasis on the location of the ITCZ and mid- and high latitudes heat budget. Clim. Past, 3(2):279-296, 2007 b.

P. Braconnot, S. P. Harrison, M. Kageyama, P. J. Bartlein, V. Masson-Delmotte, A. AbeOuchi, B. Otto-Bliesner, and Y. Zhao. Evaluation of climate models using palaeoclimatic data. Nature Climate Change, 2:417-424, 2012. 
C. E. Brennan, A. J. Weaver, M. Eby, and K. J. Meissner. Modelling oxygen isotopes in the University of Victoria Earth System Climate Model. Geoscientific Model Development Discussions, 4(3):2545-2576, 2011. doi: 10.5194/gmdd-4-2545-2011. Unpublished discussion paper.

D. Bromwich, R. Fogt, and K. Hodges. A tropospheric assessment of the ERA-40, NCEP, and JRA-25 global reanalyses in the polar regions. J. Geophys. Res., 112:D10111, 2007.

V. Brovkin, J. Bendtsen, M. Claussen, A. Ganopolski, C. Kubatzki, V. Petoukhov, and A. Andreev. Carbon cycle, vegetation, and climate dynamics in the Holocene: Experiments with the CLIMBER-2 model. Global Biogeochem. Cycles, 16(4):1139, 2002a.

V. Brovkin, M. Hofmann, J. Bendtsen, and A. Ganopolski. Ocean biology could control atmospheric $\delta^{13} \mathrm{C}$ during glacial-interglacial cycle. Geochem. Geophys. Geosyst., 3(5): 1027, 2002b.

V. Brovkin, A. Ganopolski, D. Archer, and S. Rahmstorf. Lowering of glacial atmospheric $\mathrm{CO}_{2}$ in response to changes in oceanic circulation and marine biogeochemistry. Paleoceanography, 22(4):PA4202, 2007.

A. Burke, O. Marchal, L. I. Bradtmiller, J. F. Mcmanus, and R. Francois. Application of an inverse method to interpret ${ }^{231} \mathrm{~Pa} /{ }^{230} \mathrm{Th}$ observations from marine sediments. Paleoceanography, 26(1):PA1212, 2011.

S. J. Burns and M. A. Maslin. Composition and circulation of bottom water in the western Atlantic Ocean during the last glacial, based on pore-water analyses from the Amazon Fan. Geology, 27(11):1011-1014, 1999.

E. P. Chassignet and D. P. Marshall. Gulf Stream separation in numerical ocean models. In Geophys. Monogr. Ser., pages 39-61. AGU, Washington, DC, 2008.

P. Clark, A. Dyke, J. Shakun, A. Carlson, J. Clark, B. Wohlfarth, J. Mitrovica, S. Hostetler, and A. McCabe. The Last Glacial Maximum. Science, 325(5941):710-714, 2009.

P. U. Clark and A. C. Mix. Ice sheets and sea level of the Last Glacial Maximum. Quaternary Sci. Rev., 21(1-3):1-7, 2002.

S. Clemens. Dust response to seasonal atmospheric forcing: Proxy evaluation and calibration. Paleoceanography, 13(5):471-490, 1998.

CLIMAP Project Members. The Surface of the Ice-Age Earth. Science, 191(4232):11311137, 1976.

M. Conte, M.-A. Sicre, C. Rühlemann, J. Weber, S. Schulte, D. Schulz-Bull, and T. Blanz. Global temperature calibration of the alkenone unsaturation index $\left(\mathrm{U}_{37}^{K^{\prime}}\right)$ in surface waters and comparison with surface sediments. Geochem. Geophys. Geosyst., 7(2):Q02005, 2006. 
K. H. Cook and I. M. Held. The stationary response to large-scale orography in a general circulation model and a linear model. J. Atmospheric Sci., 49(6):525-539, 1992.

P. Courtier. Dual formulation of four-dimensional variational assimilation. Quart. J. Roy. Meteor. Soc., 123:2449-2461, 1997.

W. B. Curry and D. W. Oppo. Glacial water mass geometry and the distribution of $\delta^{13} \mathrm{C}$ of $\Sigma \mathrm{CO}_{2}$ in the western Atlantic Ocean. Paleoceanography, 20:A1017+, 2005.

B. Dale. The sedimentary record of dinoflagellate cysts: looking back into the future of phytoplankton blooms. Scientia Marina, 65(S2):257-272, 2001.

G. Danabasoglu. A comparison of global ocean general circulation model solutions obtained with synchronous and accelerated integration methods. Ocean Modelling, 7(3-4): 323-341, 2004.

A. De Vernal, C. Hillaire-Marcel, and J. Turon. Reconstruction of sea-surface temperature, salinity, and sea-ice cover in the northern North Atlantic during the Last Glacial Maximum based on dinocyst assembages. Cananadian J. Earth Sci., 37:725-750, 2000.

A. De Vernal, C. Hillaire-Marcel, and W. Peltier. Structure of the upper water column in the northwest North Atlantic: Modern versus Last Glacial Maximum conditions. Paleoceanography, 17(4):1050, 2002.

A. De Vernal, F. Eynaud, M. Henry, C. Hillaire-Marcel, L. Londeix, S. Mangin, J. Matthiessen, F. Marret, T. Radi, A. Rochon, S. Solignac, and J.-L. Turon. Reconstruction of sea-surface conditions at middle to high latitudes of the Northern Hemisphere during the Last Glacial Maximum (LGM) based on dinoflagellate cyst assemblages. Quaternary Sci. Rev., 24(7-9):897-924, 2005.

A. De Vernal, A. Rosell-Melé, M. Kucera, C. Hillaire-Marcel, F. Eynaud, M. Weinelt, T. Dokken, and M. Kageyama. Comparing proxies for the reconstruction of LGM seasurface conditions in the northern North Atlantic. Quaternary Sci. Rev., 25(21-22):28202834, 2006.

D. P. Dee, S. M. Uppala, A. J. Simmons, P. Berrisford, P. Poli, S. Kobayashi, U. Andrae, M. A. Balmaseda, G. Balsamo, P. Bauer, P. Bechtold, A. C. M. Beljaars, L. van de Berg, J. Bidlot, N. Bormann, C. Delsol, R. Dragani, M. Fuentes, A. J. Geer, L. Haimberger, S. B. Healy, H. Hersbach, E. V. Hólm, L. Isaksen, P. Kållberg, M. Köhler, M. Matricardi, A. P. McNally, B. M. Monge-Sanz, J.-J. Morcrette, B.-K. Park, C. Peubey, P. de Rosnay, C. Tavolato, J.-N. Thépaut, and F. Vitart. The ERA-Interim reanalysis: configuration and performance of the data assimilation system. Q. J. Roy. Meteor. Soc., 137(656):553-597, 2011. 
B. Delmonte, J. Petit, K. Andersen, I. Basile-Doelsch, V. Maggi, and V. Y. Lipenkov. Dust size evidence for opposite regional atmospheric circulation changes over east Antarctica during the last climatic transition. Clim. Dynam., 23:427 - 438, 2004.

G. Duchemin, F. J. Jorissen, F. Le Loc'h, F. Andrieux-Loyer, C. Hily, and G. Thouzeau. Seasonal variability of living benthic foraminifera from the outer continental shelf of the Bay of Biscay. J. Sea Research, 59(4):297-319, 2008.

J.-C. Duplessy, L. Labeyrie, and C. Waelbroeck. Constraints on the ocean oxygen isotopic enrichment between the Last Glacial Maximum and the Holocene: Paleoceanographic implications. Quaternary Sci. Rev., 21(1-3):315-330, 2002.

M. Ekstrom, G. H. McTainsh, and A. Chappell. Australian dust storms: temporal trends and relationships with synoptic pressure distributions (1960-99). Int. J. Climatology, 24 (12):1581-1599, 2004.

H. Evans and I. Hall. Deepwater circulation on Blake Outer Ridge (western North Atlantic) during the Holocene, Younger Dryas, and Last Glacial Maximum. Geochem. Geophys. Geosyst., 9(3):Q03023, 2008.

R. G. Fairbanks. A 17,000-year glacio-eustatic sea level record: influence of glacial melting rates on the Younger Dryas event and deep-ocean circulation. Nature, 342(6250):637$642,1989$.

E. Farmer, A. Kaplan, P. de Menocal, and J. Lynch-Stieglitz. Corroborating ecological depth preferences of planktonic foraminifera in the tropical Atlantic with the stable oxygen isotope ratios of core top specimens. Paleoceanography, 22:PA3205, 2007.

I. Fenty and P. Heimbach. Coupled sea ice-ocean state estimation in the Labrador Sea and Baffin Bay. J. Phys. Oceanogr., 2012. in review.

I. G. Fenty. State estimation of the Labrador Sea with a coupled sea ice-ocean adjoint model. PhD thesis, Massachusetts Institute of Technology, 2010.

D. Florineth and C. Schluchter. Alpine evidence for atmospheric circulation patterns in Europe during the Last Glacial Maximum. Quaternary Res., 54:295 - 308, 2000.

G. Forget. Mapping ocean observations in a dynamical framework: A 2004-06 ocean atlas. J. Phys. Oceanogr., 40:1201-1221, 2010.

G. Forget and C. Wunsch. Estimated global hydrographic variability. J. Phys. Oceanogr., 37:1997-2008, 2007.

M. Frank. Geochemical proxies of ocean circulation and weathering inputs: Radiogenic isotopes of $\mathrm{Nd}, \mathrm{Pb}, \mathrm{Sr}, \mathrm{Hf}$, and Os. IOP Conference Series: Earth and Environmental Science, 14(1):7, 2011. 
A. Ganachaud. Large-scale mass transports, water mass formation, and diffusivities estimated from World Ocean Circulation Experiment (WOCE) hydrographic data. J. Geophys. Res., 108(C7):3213, 2003.

A. Ganachaud and C. Wunsch. Large-scale ocean heat and freshwater transports during the World Ocean Circulation Experiment. J. Climate, 16:696-705, 2003.

P. Gaspar, Y. Gregoris, and J. M. Lefevre. A simple eddy kinetic energy model for simulations of the oceanic vertical mixing: Tests at station papa and long-term upper ocean study site. J. Geophys. Res., 95(C9):179-192, 1990.

G. Gebbie. Subduction in an Eddy-Resolving State Estimate of the Northeast Atlantic Ocean. PhD thesis, MIT/WHOI Joint Program, 2004.

G. Gebbie and P. Huybers. Meridional circulation during the Last Glacial Maximum explored through a combination of South Atlantic $\delta^{18} \mathrm{O}$ observations and a geostrophic inverse model. Geochem. Geophys. Geosyst., 7(11):Q11N07, 2006.

G. Gebbie and P. Huybers. Total matrix intercomparison: A method for determining the geometry of water-mass pathways. J. Phys. Oceanogr., 40(8):1710-1728, 2010.

G. Gebbie and P. Huybers. The mean age of ocean waters inferred from radiocarbon observations: Sensitivity to surface sources and accounting for mixing histories. J. Phys. Oceanogr., 42(2):291-305, 2012.

G. Gebbie, P. Heimbach, and C. Wunsch. Strategies for nested and eddy-permitting state estimation. J. Geophys. Res., 111:C10073, 2006.

P. Gent and J. McWilliams. Isopycnal mixing in ocean circulation models. J. Phys. Oceanogr., 20:150-155, 1990.

R. Giering and T. Kaminski. Recipes for adjoint code construction. ACM Trans. Math. Softw., 24(4):437-474, 1998.

R. Giering, T. Kaminski, and T. Slawig. Generating efficient derivative code with TAF: adjoint and tangent linear Euler flow around an airfoil. Future Gener. Comp. Sy., 21(8): 1345-1355, 2005.

J. C. Gilbert and C. LeMarechal. Some numerical experiments with variable-storage quasiNewton algorithms. Mathematical Programming, 45:407-435, 1989.

S. Griffies, A. Biastoch, C. Boning, F. Bryan, G. Danabasoglu, E. Chassignet, M. England, R. Gerdes, H. Haak, R. Hallberg, W. Hazeleger, J. Jungclaus, W. Large, G. Madec, A. Pirani, B. Samuels, M. Scheinert, A. Sen Gupta, C. Severijns, H. Simmons, A. Treguier, M. Winton, S. Yeager, and J. Yin. Coordinated ocean-ice reference experiments (cores). Ocean Modelling, 26(1-2):1 - 46, 2009. 
F. E. Grousset and P. E. Biscaye. Tracing dust sources and transport patterns using Sr, Nd, and $\mathrm{Pb}$ isotopes. Chem. Geol., 222(3-4):149-167, 2005.

N. Gruber, C. D. Keeling, R. B. Bacastow, P. R. Guenther, T. J. Lueker, M. Wahlen, H. A. Meijer, W. G. Mook, and T. F. Stocker. Spatiotemporal patterns of carbon-13 in the global surface oceans and the oceanic Suess effect. Global Biogeochem. Cycles, 13(2): 307-335, 1999.

T. Hanebuth, K. Stattegger, and P. M. Grootes. Rapid Flooding of the Sunda Shelf: A Late-Glacial Sea-Level Record. Science, 288(5468):1033-1035, 2000.

J. Hargreaves, A. Paul, R. Ohgaito, A. Abe-Ouchi, and J. D. Annan. Are paleoclimate model ensembles consistent with the MARGO data synthesis? Clim. Past, 7(3):917933, 2011.

A. Hayes, M. Kucera, N. Kallel, L. Sbaffi, and E. Rohling. Glacial Mediterranean sea surface temperatures based on planktonic foraminiferal assemblages. Quaternary Sci. Rev., 24(7-9):999-1016, 2005.

P. Heimbach and V. Bugnion. Greenland ice-sheet volume sensitivity to basal, surface and initial conditions derived from an adjoint model. Annals of Glaciology, 50(52):67-80, 2009.

P. Heimbach, C. Hill, and R. Giering. An efficient exact adjoint of the parallel MIT general circulation model, generated via automatic differentiation. Future Gener. Comp. Sy., 21 (8):1356-1371, 2005.

P. P. Hesse and G. H. McTainsh. Last Glacial Maximum to Early Holocene wind strength in the mid-latitudes of the Southern Hemisphere from aeolian dust in the Tasman Sea. Quaternary Res., 52:343-349, 1999.

T. Hesse, M. Butzin, T. Bickert, and G. Lohmann. A model-data comparison of $\delta^{13} \mathrm{C}$ in the glacial Atlantic Ocean. Paleoceanography, 26(3):PA3220, 2011.

C. Jackson. Sensitivity of stationary wave amplitude to regional changes in Laurentide ice sheet topography in single-layer models of the atmosphere. J. Geophys. Res., 105: 24443-24454, 2000.

C. Jeandel, T. Arsouze, F. Lacan, P. Techine, and J.-C. Dutay. Isotopic Nd compositions and concentrations of the lithogenic inputs into the ocean: A compilation, with an emphasis on the margins. Chem. Geol., 239(1-2):156 - 164, 2007.

K. M. Jones, S. P. Khatiwala, S. L. Goldstein, S. R. Hemming, and T. van de Flierdt. Modeling the distribution of $\mathrm{Nd}$ isotopes in the oceans using an ocean general circulation model. Earth Planet. Sci. Lett., 272(3-4):610-619, 2008. 
S. Joussaume and K. E. Taylor. Status of the Paleoclimate Modeling Intercomparison Project (PMIP). In Proc. of the first international AMIP scientific conference, WCRP Report, pages 425-430, 1995.

R. E. Kalman. A new approach to linear filtering and prediction problems. Transactions of the ASME - J. of Basic Engineering, 82(Series D):35-45, 1960.

E. Kalnay and coauthors. The NCEP/NCAR 40-Year Reanalysis Project. Bull. Amer. Metero. Soc., 77:437-471, 1996.

L. Keigwin and E. Boyle. Did North Atlantic overturning halt 17,000 years ago? Paleoceanography, 23:PA1101, 2008.

R. Key, A. Kozyr, C. Sabine, K. Lee, R. Wanninkhof, J. Bullister, R. Feely, F. Millero, C. Mordy, and T. Peng. A global ocean carbon climatology: Results from Global Data Analysis Project (GLODAP). Global Biogeochem. Cycles, 18:GB4031, 2004.

R. Key, T. Tanhua, A. Olsen, M. Hoppema, S. Jutterstrom, C. Schirnick, S. van Heuven, A. Kozyr, X. Lin, A. Velo, D. Wallace, and L. Mintrop. The CARINA data synthesis project: introduction and overview. Earth Syst. Sci. Data, 2:105-121, 2010.

K. E. Kohfeld and S. P. Harrison. How well can we simulate past climates? Evaluating models using global palaeoenvironmental datasets. Quaternary Sci. Rev., 19:321-346, 2000.

K. E. Kohfeld and S. P. Harrison. DIRTMAP: the geological record of dust. Earth-Sci. Rev., 54(1-3):81-114, 2001.

P. Kroopnick. The distribution of $\delta^{13} \mathrm{C}$ of $\Sigma \mathrm{CO}_{2}$ in the world oceans. Deep Sea Res. Part A, 32:57-84, 1985.

M. Kucera, A. Rosell-Melé, R. Schneider, C. Waelbroeck, and M. Weinelt. Multiproxy approach for the reconstruction of the glacial ocean surface (MARGO). Quaternary Sci. Rev., 24(7-9):813-819, 2005a.

M. Kucera, M. Weinelt, T. Kiefer, U. Pflaumann, A. Hayes, M. Weinelt, M.-T. Chen, A. C. Mix, T. Barrows, E. Cortijo, J. Duprat, S. Juggins, and C. Waelbroeck. Reconstruction of sea-surface temperatures from assemblages of planktonic foraminifera: multi-technique approach based on geographically constrained calibration data sets and its application to glacial Atlantic and Pacific Oceans. Quaternary Sci. Rev., 24(7-9):951-998, 2005b.

F. Lacan, K. Tachikawa, and C. Jeandel. Neodymium isotopic composition of the oceans: A compilation of seawater data. Chem. Geol., 300-301(0):177-184, 2012.

W. Large and S. Yeager. Diurnal to decadal global forcing for ocean and sea-ice models: The data sets and flux climatologies. Technical report, NCAR TN-4601STR, 2004. 
P. LeGrand and C. Wunsch. Constraints from paleotracer data on the North Atlantic circulation during the Last Glacial Maximum. Paleoceanography, 10(6):1011-1045, 1995.

A. LeGrande and G. A. Schmidt. Global gridded data set of the oxygen isotopic composition in seawater. Geophys. Res. Lett., 33:L12604, 2006.

C. Li and D. S. Battisti. Reduced Atlantic storminess during Last Glacial Maximum: Evidence from a coupled climate model. J. Climate, 21(14):3561-3579, 2008.

A. C. Lorenc. Analysis of methods for numerical weather prediction. Q. J. R. Meterol. Soc., 112:1177-1194, 1986.

M. Losch, D. Menemenlis, J. M. Campin, P. Heimbach, and C. Hill. On the formulation of sea-ice models. Part 1: Effects of different solver implementations and parameterizations. Ocean Modeling, 33:129-144, 2010.

M. S. Lozier. Overturning in the North Atlantic. Annu. Rev. Mar. Sci., 4:291-315, 2012.

D. Lund, J. F. Adkins, and R. Ferrari. Abyssal Atlantic circulation during the Last Glacial Maximum: Constraining the ratio between transport and vertical mixing. Paleoceanography, 26(1):PA1213, 2011.

J. Lynch-Stieglitz. Tracers of past ocean circulation. In H. Elderfield, editor, Treatise on Geochemistry, Vol. 6, pages 433-451. Elsevier, 2003.

J. Lynch-Stieglitz, W. B. Curry, and N. Slowey. A geostrophic transport estimate for the Florida Current from the oxygen isotope composition of benthic foraminifera. Paleoceanography, 14(3):360-373, 1999a.

J. Lynch-Stieglitz, W. B. Curry, and N. Slowey. Weaker Gulf Stream in the Florida straits during the Last Glacial Maximum. Nature, 402:644-648, 1999 b.

J. Lynch-Stieglitz, W. B. Curry, D. Oppo, U. Ninneman, C. Charles, and J. Munson. Meridional overturning circulation in the South Atlantic at the Last Glacial Maximum. Geochem. Geophys. Geosyst., 7(10):Q10N03, 2006.

N. Mahowald, K. Kohfeld, M. Hansson, Y. Balkanski, S. Harrison, I. Prentice, M. Schulz, and H. Rodhe. Dust sources and deposition during the Last Glacial Maximum and current climate: A comparison of model results with paleodata from ice cores and marine sediments. J. Geophys. Res., 104(D13):15895-15916, 1999.

N. Mahowald, D. Muhs, S. Levis, P. Rasch, M. Yoshioka, C. Zender, and C. Luo. Change in atmospheric mineral aerosols in response to climate: Last glacial period, preindustrial, modern, and doubled carbon dioxide climates. J. Geophys. Res., 111:D10202, 2006. 
G. Maier and R. Scholger. Demonstration of connection between pollutant dispersal and atmospheric boundary layers by use of magnetic susceptibility mapping, St. Jacob (Austria). Physics and Chemistry of the Earth, Parts A/B/C, 29(13-14):997-1009, 2004.

S. Manabe and A. Broccoli. The influence of continental ice sheets on the climate of an ice-age. J. Geophys. Res., 90(D1):2167-2190, 1985.

B. Manighetti and I. McCave. Late Glacial and Holocene palaeocurrents around Rockall Bank, NE Atlantic Ocean. Paleoceanography, 10(3):611-626, 1995.

O. Marchal and W. B. Curry. On the abyssal circulation in the glacial Atlantic. J. Phys. Oceanogr., 38(9):2014-2037, 2008.

T. M. Marchitto and W. S. Broecker. Deep water mass geometry in the glacial Atlantic Ocean: A review of constraints from the paleonutrient proxy $\mathrm{Cd}$. Geochem. Geophys. Geosyst., 7(12):Q12003, 2006.

J.-C. Mareschal and H. Beltrami. Evidence for recent warming from perturbed geothermal gradients: examples from eastern Canada. Clim. Dynam., 6(3-4):135-143, 1992.

J. Marshall, A. Adcroft, C. Hill, L. Perelman, and C. Heisey. A finite-volume, incompressible Navier Stokes model for studies of the ocean on parallel computers. J. Geophys. Res., 102(C3):5753-5766, 1997.

J. Maurer. Atlas of the cryosphere. Boulder, Colorado USA: National Snow and Ice Data Center. Digital media., 2007.

M. Mazloff, P. Heimbach, and C. Wunsch. An eddy-permitting Southern Ocean state estimate. J. Phys. Oceanogr., 40:880-899, 2010.

I. McCave. Chapter one deep-sea sediment deposits and properties controlled by currents. In C. Hillaire-Marcel and A. De Vernal, editors, Proxies in Late Cenozoic Paleoceanography, volume 1 of Developments in Marine Geology, pages 19-62. Elsevier, 2007.

I. McCave and I. Hall. Size sorting in marine muds: Processes, pitfalls, and prospects for paleoflow-speed proxies. Geochem. Geophys. Geosyst., 7(10):Q10N05, 2006.

I. McCave, B. Manighetti, and N. Beveridge. Circulation in the glacial North Atlantic inferred from grain-size measurements. Nature, 374:149-152, 1995.

R. McDuff. The chemistry of interstitial waters, Deep Sea Drilling Project Leg 86, volume DSDP, 86. U.S. Government Printing Office, Washington, D.C., 1985.

J. F. Mcmanus, R. Francois, J. M. Gherardi, L. Keigwin, and S. Brown-Leger. Collapse and rapid resumption of Atlantic meridional circulation linked to deglacial climate changes. Nature, 428(6985):834-837, 2004. 
C. Meinen, M. Baringer, and S. Garzoli. Variability in Deep Western Boundary Current transports: preliminary results from $26.5^{\circ} \mathrm{N}$ in the Atlantic. Geophys. Res. Lett., 33(17): L17610, 2006.

M. Meland, E. Jansen, and H. Elderfield. Constraints on SST estimates for the northern North Atlantic/Nordic Seas during the LGM. Quaternary Sci. Rev., 24(7-9):835-852, 2005.

A. Morey, A. C. Mix, and N. Pisias. Planktonic foraminiferal assemblages preserved in surface sediments correspond to multiple environment variables. Quaternary Sci. Rev., 24(7-9):813-1107, 2005.

S. Mulitza, B. Donner, G. Fischer, and A. Paul. The South Atlantic oxygen isotope record of planktic foraminifera. In G. Wefer, S. Mulitza, and V. Ratmeyer, editors, The South Atlantic in the Late Quaternary: Reconstruction of Material Budgets and Current Systems, pages 121-142. Springer, 2003.

G. N'Tchayi, J. Bertrand, M. Legrand, and J. Baudet. Temporal and spatial variations of the atmospheric dust loading throughout West Africa over the last thirty years. Ann. Geophys., 12:265-273, 1994.

K. Oliver, B. Hoogakker, S. Crowhurst, G. Henderson, R. Rickaby, N. Edwards, and H. Elderfield. A synthesis of marine sediment core $\delta^{13} \mathrm{C}$ data over the last 150000 years. Clim. Past, 6:645-673, 2010.

D. Ostermann and W. B. Curry. Calibration of stable isotopic data: an enriched $\delta^{18} \mathrm{O}$ standard used for source gas mixing detection and correction. Paleoceanography, 15(3): 353-360, 2000.

B. L. Otto-Bliesner, E. C. Brady, G. Clauzet, R. Tomas, S. Levis, and Z. Kothavala. Last Glacial Maximum and Holocene climate in CCSM3. J. Climate, 19(11):2526-2544, 2006.

B. L. Otto-Bliesner, C. Hewitt, T. M. Marchitto, E. C. Brady, A. Abe-Ouchi, M. Crucifix, S. Murakami, and S. L. Weber. Last Glacial Maximum ocean thermohaline circulation: PMIP2 model intercomparisons and data constraints. Geophys. Res. Lett., 34(L12706), 2007.

B. L. Otto-Bliesner, R. Schneider, E. C. Brady, M. Kucera, A. Abe-Ouchi, E. Bard, P. Braconnot, M. Crucifix, C. D. Hewitt, M. Kageyama, O. Marti, A. Paul, A. Rosell-Melé, C. Waelbroeck, S. L. Weber, and Y. Yu. A comparison of PMIP2 model simulations and the MARGO proxy reconstruction for tropical sea surface temperatures at Last Glacial Maximum. Clim. Dynam., 32(6):799-815, 2009. 
D. Parkin and R. Padgham. Further studies on trade winds during the glacial cycles. Proc. of the Royal Society of London. Series A, Mathematical and Physical Sciences, 346(1645): 245-260, 1975.

A. Paul and S. Mulitza. Challenges to understanding ocean circulation during the Last Glacial Maximum. Eos Trans. AGU, 90(19):169, 2009.

A. Paul, S. Mulitza, J. Patzold, and T. Wolff. Simulation of oxygen isotopes in a global ocean model. In G. Fischer and G. Wefer, editors, Use of Proxies in Paleoceanography: Examples from the South Atlantic, pages 655-686. Springer-Verlag, 1999.

W. R. Peltier. Global glacial isostasy and the surface of the ice-age earth: The ICE-5G (VM2) model and GRACE. Annual Review of Earth and Planetary Sciences, 32:111149, 2004.

U. Pflaumann, M. Sarnthein, and M. Chapman. Glacial North Atlantic: sea-surface conditions reconstructed by GLAMAP 2000. Paleoceanography, 18(3):1065, 2003.

P. Quay, R. Sonnerup, J. Stutsman, J. Maurer, A. Kortzinger, X. A. Padin, and C. Robinson. Anthropogenic $\mathrm{CO}_{2}$ accumulation rates in the North Atlantic Ocean from changes in the ${ }^{13} \mathrm{C}^{12} \mathrm{C}$ of dissolved inorganic carbon. Global Biogeochem. Cycles, 21:GB1009, 2007.

D. K. Rea. The paleoclimate record provided by eolian deposition in the deep sea: the geologic history of wind. Rev. Geophys., 32(2):159-195, 1994.

M. Redi. Oceanic isopycnal mixing by coordinate rotation. J. Phys. Oceanogr., 12:1154$1158,1982$.

P. Reimer and coauthors. IntCal04 terrestrial radiocarbon age calibration, 0-26 cal kyr BP. Radiocarbon, 46:1029-1058, 2004.

M. J. Roberts, H. Banks, N. Gedney, J. Gregory, R. Hill, S. Mullerworth, A. Pardaens, G. Rickard, R. Thorpe, and R. Wood. Impact of an eddy-permitting ocean resolution on control and climate change simulations with a global coupled GCM. J. Climate, 17(1): 3-20, 2004.

D. Roche, D. Paillard, A. Ganopolski, and G. Hoffmann. Oceanic oxygen-18 at the present day and LGM: equilibrium simulations with a coupled climate model of intermediate complexity. Earth Planet. Sci. Lett., 218:318-330, 2004.

G. H. Roe and R. S. Lindzen. The mutual interaction between continental-scale ice sheets and atmospheric stationary waves. J. Climate, 14(7):1450-1465, 2001.

M. Rojas, P. Moreno, M. Kageyama, M. Crucifix, C. Hewitt, A. Abe-Ouchi, R. Ohgaito, E. C. Brady, and P. Hope. The Southern Westerlies during the Last Glacial Maximum in PMIP2 simulations. Clim. Dynam., 32(4):525-548, 2009. 
A. Rosell-Melé and P. Comes. Evidence for a warm Last Glacial Maximum in the Nordic Seas or an example of shortcomings in $U_{37}^{K^{\prime}}$ and $U_{37}^{K}$ to estimate low sea surface temperature? Paleoceanography, 14(6):770-776, 1999.

A. Rosell-Melé, E. Bard, K.-C. Emeis, B. Grieger, C. Hewitt, P. Müller, and R. Schneider. Sea surface temperature anomalies in the oceans at the LGM estimated from the alkenone- $U_{37}^{K^{\prime}}$ index: comparison with GCMs. Geophys. Res. Lett., 31(3):L03208, 2004.

A. Sanyal, N. Hemming, G. Hanson, and W. S. Broecker. Evidence for a higher pH in the glacial ocean from boron isotopes in foraminifera. Nature, 373:234-236, 1995.

M. Sarnthein, R. Gersonde, S. Niebler, U. Pflaumann, R. Spielhagen, J. Thiede, G. Wefer, and M. Weinelt. Overview of Glacial Atlantic Ocean Mapping (GLAMAP 2000). Paleoceanography, 18(2):1030, 2003.

J. J. Schanze, R. W. Schmitt, and L. L. Yu. The global oceanic freshwater cycle: A stateof-the-art quantification. J. Mar. Res., 68:569-595, 2010.

R. Schiebel and C. Hemleben. Modern planktic foraminifera. Paläontologische Zeitschrift, 79(1):135-148, 2005.

G. Schmidt, G. Bigg, and E. Rohling. Global Seawater Oxygen-18 Database. http://data.giss.nasa.gov/ol8data, 1999.

G. A. Schmidt. Enhancing the relevance of palaeoclimate model/data comparisons for assessments of future climate change. J. Quaternary Sci., 25(1):79-87, 2010.

G. A. Schmidt, A. N. LeGrande, and G. Hoffmann. Water isotope expressions of intrinsic and forced variability in a coupled ocean-atmosphere model. J. Geophys. Res., 112: D10103, 2007.

J. Schmitt, R. Schneider, J. Elsig, D. Leuenberger, A. Lourantou, J. Chappellaz, P. Kohler, F. Joos, T. F. Stocker, M. Leuenberger, and H. Fischer. Carbon isotope constraints on the deglacial $\mathrm{CO}_{2}$ rise from ice cores. Science, 336(6082):711-714, 2012.

A. Schmittner, N. M. Urban, J. D. Shakun, N. M. Mahowald, P. U. Clark, P. J. Bartlein, A. C. Mix, and A. Rosell-Melé. Climate sensitivity estimated from temperature reconstructions of the Last Glacial Maximum. Science, 334:1385-1388, 2011.

B. Schneider, G. Leduc, and W. Park. Disentangling seasonal signals in Holocene climate trends by satellite-model-proxy integration. Paleoceanography, 25(4):PA4217, 2010.

D. P. Schrag and D. J. DePaolo. Determination of $\delta^{18} \mathrm{O}$ of seawater in the deep ocean during the Last Glacial Maximum. Paleoceanography, 8:1-6, 1993.

D. P. Schrag, G. Hampt, and D. W. Murray. Pore fluid constraints on the temperature and oxygen isotopic composition of the glacial ocean. Science, 272(5270):1930-1932, 1996. 
D. P. Schrag, J. F. Adkins, K. McIntyre, J. Alexander, D. Hodell, C. D. Charles, and J. F. Mcmanus. The oxygen isotopic composition of seawater during the Last Glacial Maximum. Quaternary Sci. Rev., 21(1-3):331-342, 2002.

N. Shackleton. Attainment of isotopic equilibrium between ocean water and the benthonic foraminifera genus Uvigerina: isotopic changes in the ocean during the last glacial. Les méthodes quantitatives d'étude des variations du climat au cours du Pleistocene, Gifsur-Yvette, Colloque international du CNRS, 219:203-210, 1974.

J. D. Shakun, P. U. Clark, F. He, S. A. Marcott, A. C. Mix, Z. Liu, B. Otto-Bliesner, A. Schmittner, and E. Bard. Global warming preceded by increasing carbon dioxide concentrations during the last deglaciation. Nature, 484(7392):49-54, 2012.

S. I. Shin, Z. Liu, B. L. Otto-Bliesner, E. C. Brady, J. Kutzbach, and S. Harrison. A simulation of the Last Glacial Maximum climate using the NCAR-CCSM. Clim. Dynam., 20 (2):127-151, 2003.

M. J. Siegert. Ice Sheets and Late Quaternary Environmental Change. John Wiley \& Sons, Chichester, England, 2001.

S. Solomon, D. Qin, M. Manning, Z. Chen, M. Marquis, K. Averyt, M. Tignor, and H. e. Miller, editors. Contribution of Working Group I to the Fourth Assessment Report of the Intergovernmental Panel on Climate Change. Cambridge University Press, Cambridge, United Kingdom and New York, NY, USA, 2007.

D. Stammer, C. Wunsch, R. Giering, C. Eckert, P. Heimbach, J. Marotzke, A. Adcroft, C. Hill, and J. Marshall. Global ocean circulation during 1992-1997, estimated from ocean observations and a general circulation model. J. Geophys. Res., 107:1-1, 2002.

D. Stammer, K. Ueyoshi, A. Köhl, W. G. Large, S. A. Josey, and C. Wunsch. Estimating air-sea fluxes of heat, freshwater, and momentum through global ocean data assimilation. J. Geophys. Res., 109(C5):C05023, 2004.

C. Sturm, Q. Zhang, and D. Noone. An introduction to stable water isotopes in climate models: benefits of forward proxy modelling for paleoclimatology. Clim. Past, 6(1): 115-129, 2010.

A. Tagliabue and L. Bopp. Towards understanding global variability in ocean carbon-13. Global Biogeochem. Cycles, 22(1):GB1025, 2008.

A. Tagliabue, L. Bopp, D. M. Roche, N. Bouttes, J.-C. Dutay, R. Alkama, M. Kageyama, E. Michel, and D. Paillard. Quantifying the roles of ocean circulation and biogeochemistry in governing ocean carbon-13 and atmospheric carbon dioxide at the Last Glacial Maximum. Clim. Past, 5(4):695-706, 2009. 
L. Talley, J. Reid, and P. Robbins. Data-based meridional overturning streamfunctions for the global ocean. J. Climate, 16:3213-3226, 2003.

K. E. Taylor, R. J. Stouffer, and G. A. Meehl. An overview of CMIP5 and the experiment design. Bull. Amer. Meteor. Soc., 93(4):485-498, 2012.

R. J. Telford. Limitations of dinoflagellate cyst transfer functions. Quaternary Sci. Rev., 25(13-14):1375-1382, 2006.

W. Thacker and R. Long. Fitting dynamics to data. J. Geophys. Res., 93(C2):1227-1240, 1988.

J. C. Tindall, P. J. Valdes, and L. C. Sime. Stable water isotopes in HadCM3: Isotopic signature of El Niño-Southern Oscillation and the tropical amount effect. J. Geophys. Res., 114(D4):D04111, 2009.

J. Toggweiler, J. Russel, and S. Carson. Midlatitude westerlies, atmospheric $\mathrm{CO}_{2}$, and climate change during the ice ages. Paleoceanography, 21:PA2005, 2006.

C. Waelbroeck, S. Mulitza, H. Spero, T. Dokken, T. Kiefer, and E. Cortijo. A global compilation of late Holocene planktonic foraminiferal $\delta^{18} \mathrm{O}$ : relationship between surface water temperature and $\delta^{18}$ O. Quaternary Sci. Rev., 24(7-9):853-868, 2005.

C. Waelbroeck, A. Paul, M. Kucera, A. Rosell-Melé, M. Weinelt, R. Schneider, A. C. Mix, A. Abelmann, L. Armand, E. Bard, S. Barker, T. Barrows, H. Benway, I. Cacho, M. T. Chen, E. Cortijo, X. Crosta, A. De Vernal, T. Dokken, J. Duprat, H. Elderfield, F. Eynaud, R. Gersonde, A. Hayes, M. Henry, C. Hillaire-Marcel, C. C. Huang, E. Jansen, S. Juggins, N. Kallel, T. Kiefer, M. Kienast, L. Labeyrie, H. Leclaire, L. Londeix, S. Mangin, J. Matthiessen, F. Marret, M. Meland, A. Morey, S. Mulitza, U. Pflaumann, N. G. Pisias, T. Radi, A. Rochon, E. J. Rohling, L. Sbaffi, C. Schäfer-Neth, S. Solignac, H. Spero, K. Tachikawa, and J. L. Turon. Constraints on the magnitude and patterns of ocean cooling at the Last Glacial Maximum. Nature Geoscience, 2(2):127-132, 2009.

A. Weaver and P. Courtier. Correlation modelling on the sphere using a generalized diffusion equation. Q. J. Roy. Meteor. Soc., 127(575):1815-1846, 2001.

S. L. Weber, S. S. Drijfhout, A. Abe-Ouchi, M. Crucifix, M. Eby, A. Ganopolski, S. Murakami, B. L. Otto-Bliesner, and W. R. Peltier. The modern and glacial overturning circulation in the Atlantic ocean in PMIP coupled model simulations. Clim. Past, 3(1): 51-64, 2007.

A. Winguth, D. Archer, J.-C. Duplessy, E. Maier-Reimer, and U. Mikolajewicz. Sensitivity of paleonutrient tracer distributions and deep-sea circulation to glacial boundary conditions. Paleoceanography, 14(3):304-323, 1999. 
A. Winguth, D. Archer, E. Maier-Reimer, and U. Mikolajewicz. Paleonutrient data analysis of the glacial Atlantic using an adjoint ocean general circulation model. Inverse Methods in Global Biogeochemical Cycles, AGU Geophysical Monograph Series, edited by P. Kasibhatla, M. Heimann, D. Harley, N. Mahowald, R. Prinn, and P. Rainer, pages 171183, 2000.

C. Wunsch. Discrete Inverse and State Estimation Problems: With Geophysical Fluid Applications. Cambridge University Press, Cambridge, UK, 2006.

C. Wunsch. Determining paleoceanographic circulations, with emphasis on the Last Glacial Maximum;. Quaternary Sci. Rev., 22(2-4):371-385, 2003.

C. Wunsch and P. Heimbach. The global zonally integrated ocean circulation, 1992-2006: seasonal and decadal variability. J. Phys. Oceanogr., 39:351-368, 2009.

C. Wunsch and P. Heimbach. Dynamically and kinematically consistent global ocean circulation state estimates with land and sea ice. In G. Siedler, J. Church, W. Gould, and S. Gric, editors, Ocean Circulation and Climate, 2nd Edition. Elsevier, 2012, in press.

C. Wunsch and P. Heimbach. Estimated decadal changes in the North Atlantic meridional overturning circulation and heat flux 1993-2004. J. Phys. Oceanogr., 36(11):2012-2024, 2006.

C. Wunsch and P. Heimbach. Practical global oceanic state estimation. Physica D: Nonlin. Phenom., 230(1-2):197-208, 2007.

C. Wunsch and P. Heimbach. How long to oceanic tracer and proxy equilibrium? Quat. Sci. Revs., 27:637-651, 2008.

C. Wunsch, P. Heimbach, R. Ponte, I. Fukumori, and Members ECCOGODAE Consortium. The global general circulation of the ocean estimated by the ECCO-Consortium. Oceanography, 22(2), 2009.

Y. Yokoyama, K. Lambeck, P. De Deckker, P. Johnston, and L. K. Fifield. Timing of the Last Glacial Maximum from observed sea-level minima. Nature, 406:713-716, 2000.

Y. Yung, T. Lee, C. Wang, and Y. Shieh. Dust: A diagnostic of the hydrologic cycle during the Last Glacial Maximum. Science, 271:962-963, 1996.

R. Zeebe. An explanation of the effect of seawater carbonate concentration on foraminiferal oxygen isotopes. Geochimica et Cosmochimica Acta, 63(13/14):2001-2007, 1999.

K. Q. Zhang and J. Marotzke. The importance of open-boundary estimation for an Indian Ocean GCM-Data synthesis. J. Mar. Res., 57:305-30534, 1999. 